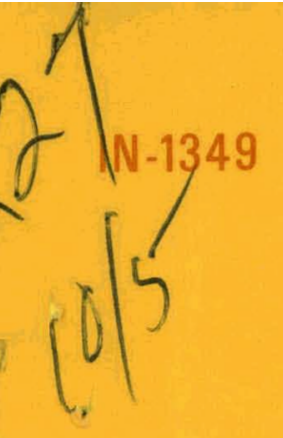

\title{
PREP AND KITT: \\ COMPUTER CODES FOR THE \\ AUTOMATIC EVALUATION OF A FAULT TREE
}

W. E. Vesely R. E. Narum

\section{IDAHO NUCLEAR CORPORATION \\ NATIONAL REACIOR IESIING STATION \\ IDAHO FALLS, IDAHO}

\section{U.S. ATOMIC ENERGY COMMISSION}




\section{DISCLAIMER}

This report was prepared as an account of work sponsored by an agency of the United States Government. Neither the United States Government nor any agency Thereof, nor any of their employees, makes any warranty, express or implied, or assumes any legal liability or responsibility for the accuracy, completeness, or usefulness of any information, apparatus, product, or process disclosed, or represents that its use would not infringe privately owned rights. Reference herein to any specific commercial product, process, or service by trade name, trademark, manufacturer, or otherwise does not necessarily constitute or imply its endorsement, recommendation, or favoring by the United States Government or any agency thereof. The views and opinions of authors expressed herein do not necessarily state or reflect those of the United States Government or any agency thereof. 


\section{DISCLAIMER}

Portions of this document may be illegible in electronic image products. Images are produced from the best available original document. 
Printed in the United States of America Available from

Clearinghouse for Federal Scientific and Technical Information National Bureau of Standards, U. S. Department of Commerce Springfield, Virginia 22151

Price: Printed Copy $\$ 3.00$; Microfiche $\$ 0.65$

\section{LEGAL NOTICE}

This report was prepared as an account of Government sponsored work. Neither the United States, nor the Commission, nor any person acting on behalf of the Commission:

A. Makes any warranty or representation, express or implied, with respect to the accuracy, completeness, or usefulness of the information contained in this report, or that the use of any information, apparatus, method, or process disclosed in this report may not infringe privately owned rights; or

B. Assumes any liabilities with respect to the use of, or for damages resulting from the use of any information, apparatus, method, or process disclosed in this report.

As used in the above, "person acting on behalf of the Commission" includes any employee or contractor of the Commission, or employee of such contractor, to the extent that such employee or contractor of the Commission, or employee of such contractor prepares, disseminates, or provides access to, any information pursuant to his employment or contract with the Commission, or his employment with such contractor. 
R. E. Narum

This report was prepared as an account of work
sponsored by the United States Government. Neither
the United States nor the United States Atomic Energy
Commission, nor any of their employees, nor any of
their contractors, subcontractors, or their employees,
makes any warranty, express or implied, or assumes any
legal liability or responsibility for the accuracy, com-
pleteness or usefulness of any information, apparatus,
product or process disclosed, or represents that its use
would not infringe privately owned rights.

would not infringe privately owned rights.

\title{
IDAHO NUCLEAR CORPORATION
}

\author{
A Jointly Owned Subsidiary of
}

AEROJET GENERAL CORPORATION

ALLIED CHEMICAL CORPORATION

PHILLIPS PETROLEUM COMPANY

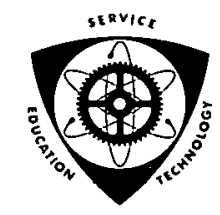

U. S. Atomic Energy Commission Scientific and Technical Report

Issued Under Contract AT(10-1)-1230

Idaho Operations Office 


\section{ABSTRACT}

A computer code package is described by which a fault tree, already constructed, can be evaluated. The fault tree may be of general complexity, may consist of up to 2000 components and 2000 gates, and may include any number of inhibit conditions. The gate structure of the fault tree is Iimited to "AND" gates and "OR" gates, and the unique components on the tree are assumed to be independent.

The code package consists of two distinct sets of computer codes: the PREP codes which obtain the minimal cut sets (failure modes) or the minimal path sets (success modes) of the fault tree and the KITT codes which obtain the numerical probabilities associated with the tree. The PREP :codes obtain the minimal cut sets or path sets either by" Monte Carlo simulation or by deterministic testing. The KITT codes obtain the numerical probabilities by means of Kinetic Tree Theory, a methodology by which exact, time-dependent probabilistic information is obtained. In the KITT codes, constant repair times, exponential repair distributions, and nonrepairability can be handled. Also, phasing can be included, where each component may have its own unique phases and the failure and repair data for the component may arbitrarily vary from phase to phase.

Input descriptions for the PREP and KI'IT codes are given, along with detailed discussions on the efficient use of the codes and the meanings and significances of the results obtained. A sample problem is also included. 


\section{PREFACE}

This report describes a computer code package written in Fortran IV for the IBM $360 / 75$ computer. The code package is to be used for the evaluation of a fault tree which has been already constructed. The fault tree; used in reliability engineering and system safety analysis, is a formal logical diagram of all the causes to a particular "top" event. The causes are the basic events which give rise to the top event and which determine the limit of resolution of the fault tree. The top event is any final event of predetermined interest, whose causes are desired. Since the report is concerned with the evaluation of the fault tree, and not its actual construction, the reader is assumed to have knowledge of the particular concepts involved in fault tree construction.

In this report, the basic events (causes) of the fault tree will be termed "component failures", and the top event of the fault tree will be termed the "system failure". The failing of the component then corresponds to the basic event occurring, and the failing of the system correspond to the top event occurring. In a complementary manner, the "functioning" state of the component corresponds to the nonexistence of the component failure (basic event), and the "functioning" state of the system corresponds to the nonexistence of the system failure (top event). In general, the "failed state" corresponds to the existence of the failure (event), and the "functioning state" corresponds to its nonexistence. The occurrence of a failure then corresponds to the passage from a functioning state to a failed state, with regard to both the system and its components.

Once constructed, the fault tree is first evaluated to obtain the unique modes by which the system failure can occur and, secondly, is evaluated to obtain the associated probability characteristics. The unique modes of occurrence are sometimes termed minimal cut sets, sometimes crit- 
ical paths, and sometimes mode failures. In this report, the terminology "minimal cut sets" will be used. A minimal cut set is a smallest group (set) of component failures which must all simultaneously exist in order for the system failure to exist. Any other component failures may coexist with this smallest set, but these other component failures are entirely redundant and do not directly cause the system failure. The finite collection of all the unique minimal cut sets of a fault tree represents all the unique, nonredundant ways by which the system failure can occur. The system failure can only occur by one of these unique ways or by various combinations of these unique ways.

Of a complementary nature to the minimal cut. sets are the minimal path. sets of the fault tree. A minimal path set is a smallest group of component failures which must not exist in order for the system failure to not exist. Any other component failures existing with the minimal path set will not cause system failure. The finite collection of all the unique minimal path sets represent all the unique ways by which the system fail= ure will not occur. The system failure will not occur if the component failures in one or more of these minimal path sets do not ocour. The fault tree can therefore also be evaluated to obtain its minimal path sets. After having obtained either the minimal cut sets or the minimal path sets, the fault tree is then secondly evaluated to obtain the probability characteristics. The minimal cut sets or the minimal path sets must be first obtained in order to obtain these characteristics. Since they are of a complementary nature, either the minimal cut sets or the minimal path sets are sufficient to obtain the probability characteristics; the same system characteristics, having the same numerical values, will be obtained from the minimal cut sets as from the minimal path sets. For most fault tree evaluations, the minimal cut sets are obtained and used to determine 
the probability characteristics since the minimal cut sets directly exhibit the ways by which the system failure can occur.

With the use of this code package, the probability characteristics, which are obtained from the minimal cut or path sets, completely quantify the fault tree. These probability characteristics include:

1. The probability of the failure existing at time $t$ (the "unavailability").

2. The probability of the failure not occurring to time $t$ (the "reliability").

3. The expected number of failures occurring to time $t$.

4. The failure rate at time $t$.

5. The failure intensity at time $t^{*}$ ("lambda").

These probability characteristics are obtained not only for the system failure, but are obtained for each component on the fault tree and for each minimal cut set or path set. From this detailed information, the importances of various components and minimal cut sets or path sets can be simply ascertained. Moreover, the characteristics are obtained at arbitrary time points $t$ specified by the user, and hence complete time behavior with respect to safety or reliability is obtained.

The input data necessary to obtain these characteristics, besides the fault tree itself, are the component failure intensities (lambdas) and repair times. The failure intensities may be constant or may vary with respect to phasing. The component failures may have constant repair times, exponential repair distributions, or may be nonrepairable. The repair data may be constant or may vary with respect to phases. Any mixture of various types of component failures can be handled, and further, any inhibit conditions may also be incorporated. The component failures on the fault tree are ascumed independent; any component failure may occur at numerous places on the fault tree but those component failures which are distinct are assumed independent. 
With this code package, fault trees of any structure may be handled, and the fault trees may be of any complexity, up to a maximun of 2000 components and 2000 gates. Even for larger fault trees the computer time needed for a complete evaluation is relatively small, with approximately one minute needed for a 500 component tree.

Because the fault tree is evaluated in two stages, first to obtain the minimal cut sets or path sets and secondly to obtain the probability characteristics, this report naturally divides into two main sections. The firsl sectlon entitled "I'he Use of the PREP Codes" describes the use of the PREP computer codes to obtain the minimal cut sets or path sets of the fault tree. The second section entitled "The Use of the KITT Codes" describes the use of the KITT computer codes to obtain the probability characteristics once the minimal cut sets or path sets have been obtained. The significance and meanings of the probability characteristics obtained are also explained in this section.

In a complete evaluation of a fault tree, the fault tree is first input in a simple coded form to the PREP computer codes to obtain the minimal cut sets or path sets. The punched output from the PREP codes, with additional component failure and repair data, is then input to the KITT codes to obtain the time-dependent, numerical probabilities. In this two-stage use, the PREP and KITT codes form one code. The codes are designed such that they may also be used independently; if the minimal cut sets or path sets are only desired then PREP may bc jingularly used, and if the minimal cut sets or path sets are already known or if additional sensitivity runs are to be analyzed with the same sets, then the KITT codes can be singularly used. In this same sense, the two sections of this report are complementary, but are also independent and self-contained. 
ABSTRACT ............................

PREFACE ............................

THE USE OF THE PREP CODES

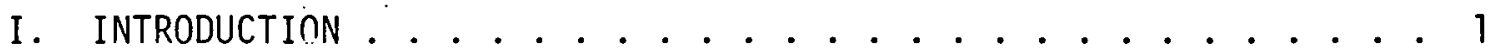

1. Fault Trees ................... 1

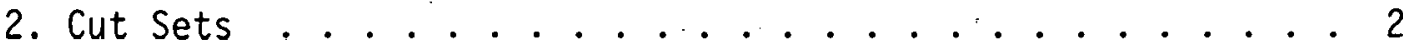

3. Path Sets................ 5

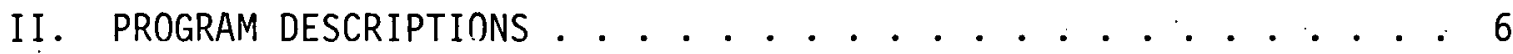

1. TREBIL . . . . . . . . . . . . . . . . . . . 6

2. MINSET ................ . . 8

Deterministic Testing (COMBO). ......... 8

Monte Carlo Simulation ............... 11

III. INPUT DESCRIPTION AND RECOMMENDATIONS . . . . . . . 15

1. Input Deck Description . . . . . . . . . . . . 15

2. Input Group 1................ 16

3. Recommendations for Input Group 1 . . . . . . . . 17

MIN . . . . . . . . . . . . . . . . . 17

$\operatorname{MAX} \ldots \ldots \ldots \ldots . \ldots 17$

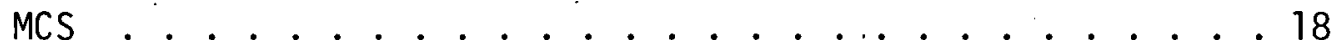

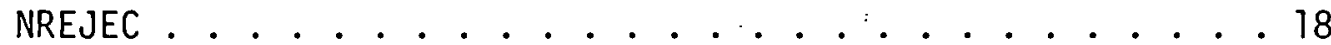

TAA ...................... 18

IREN ...................... . . 19

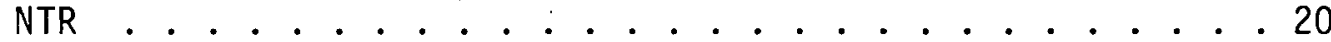

IDEX1 ..................................

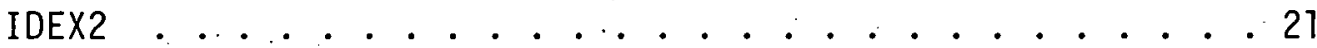

4. Input Group 2................. . . . 26

5. Recommendations for Input Group 2.......... . . . 27

6. Input Group 3 . . . . . . . . . . . . . . . . 27

7. Description and Recommendations for Input Group 3 .... 29

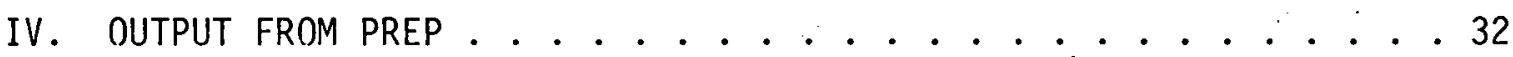

1. Output from TREBIL . . . . . . . . . . . . . 32

2. Output from MINSET . . . . . . . . . . . . . . 33 


\section{CONTENTS}

THE USE OF THE KITT CODES

KITT-1............................... 1

I. INTRODUCTION . . . . . . . . . . . . . . . . . . . 1

II. RELIABILITY INFORMATION OBTAINED BY KITT-1 . . . . . . . . . . 2

1. Component Information ................. 2

2. Minimal Cut Set Information .............. 3

3. Minimal Path Set Information . . . . . . . . . . . 5

4. System Reliability Information ............. 7

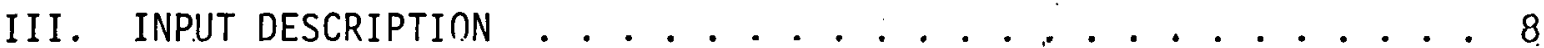

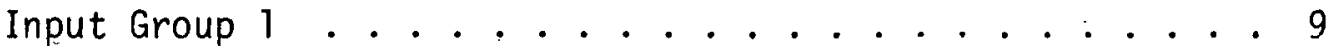

Input Group 2 . . . . . . . . . . . . . . . . . . . . 9

Input Group 3 . . . . . . . . . . . . . . . . 10

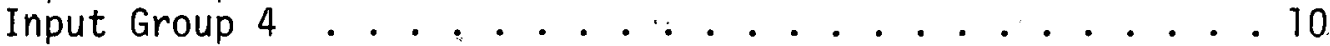

Input Group 5 . . . . . . . . . . . . . . . . . . . 11

Input Group 6 . . . . . . . . . . . . . . . . . 12

Input Group 7 . . . . . . . . . . . . . . . . . . 14

Input Group 8 . . . . . . . . . . . . . . . . . . 16

Input Group 9 .................. . . 18

a. Minimal Cut Sets Input . . . . . . . . . . . . 18

b. Minimal Path Sets Input .............. . . 23

Input Group 10 . . . . . . . . ......... 24

a. Minimal Cut Sets Input .............. 24

b. Minimal Path Sets Input . . . . . . . . . . . . 29

1. When to Cumpule the Exact Values for the System

Characteristics (ISTOP = 2) . . . . . . . . . 30

2. The Accuracy of the Upper Bound Approximations

(ISTOP = 1) ......................... 31

3. Recommendations for the Use of Bracketing

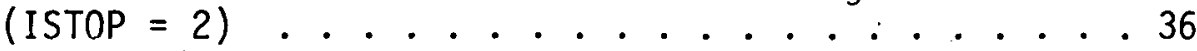

4. Recommendation Summary ............. . . 41

Input Group $11 . . . . . . . . . . . .443$

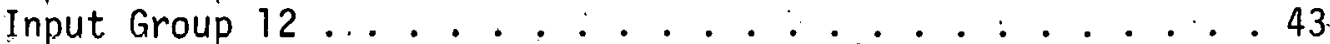

Input Group 13... . . . . . . . . . . . . . 44

Input Group 14 . . . . . . . . . . .. . . ... . 44

Input Group 15A ................. . . . . 45

Input Group 15B . . . . . . . . . . . . . . . 45

Input Group 16................... . . 46

Input Group 17A ................... . . . 47

Input Group 17B .. . . . . . . . . . . . . . 47

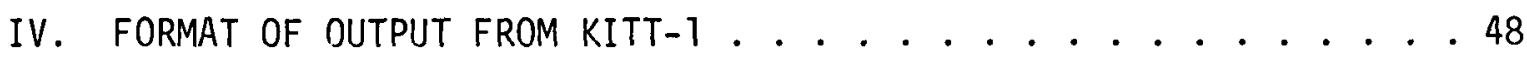


KITT-2 . . . . . . . . . . . . . . . . . . 61

I. INTRODUCTION . . . . . . . . . . . . . . . . 61

II. MULTIPHASE ILLUSTRATIONS . . . . . . . . . . . . . 63

1. The Component with a Number of Phases . . . . . . . . . 63

2. The Mock-Up of a Genera 1 Time-Dependent Failure-Intensity . . 65

3. The Mock-Up of a General Repair Distribution . . . . . . . 68

4. The Inhibit Condition With a Number of Phases . . . . . . 70

III. RELIABILITY INFORMATION OBTAINED BY KITT-2 . . . . . . . . . . 72

IV. INPUT DESCRIPTION . . . . . . . . . . . . . . . . 72

Input Group 1 . . . . . . . . . . . . . . . . 72

Input Group 2 . . . . . . . . . . . . . . . 73

Input Group 3 . . . . . . . . . . . . . . . . . . . 73

Input Group 4 . . . . . . . . . . . . . . . . . . . . 74

Input Group 5 .. . . . . . . . . . . . . . 76

Input Group 6 . . ................. . . . 77

Input Group 7 .................... 77

Input Group 8 . . . . . . . . . . . . . . . . . . 78

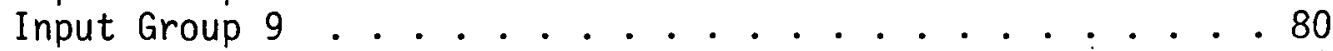

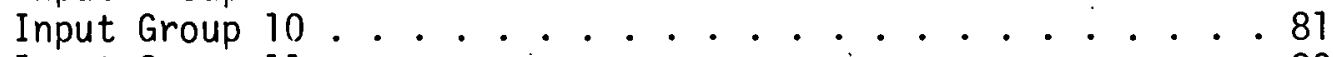

Input Group 11 . . . . . . . . . . . . . . . . 83

Input Group 12 . . . . . . . . . . . . . . . . . 84

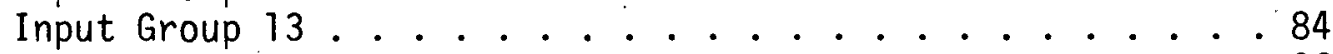

Input Group 14 . . . . . . . . . . . . . . . . . . . . 86

1. Continuation of the Characteristics by the Method of Translation................. 86

2. Continuation of the Characteristics by a Simple Point Advance .............. . . 89

Input Group 15 . . . . . . . . . . . . . . . 92

Input Group 16 . . . . . . . .. . . . . . . . 92

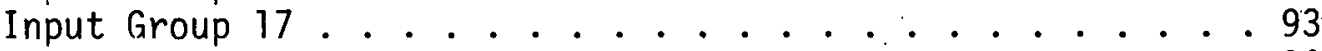

Input Group 18 . . . . . . . . . . . . . . . . 94

Input Group 19..................... . . . . 94

Input Group 20 ........................... 94

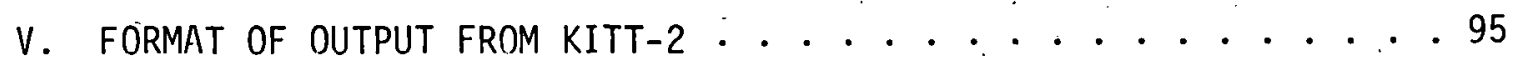

VI. REFERENCES . . . . . . . . . . . . . . . . . . . 99 
CONTENTS

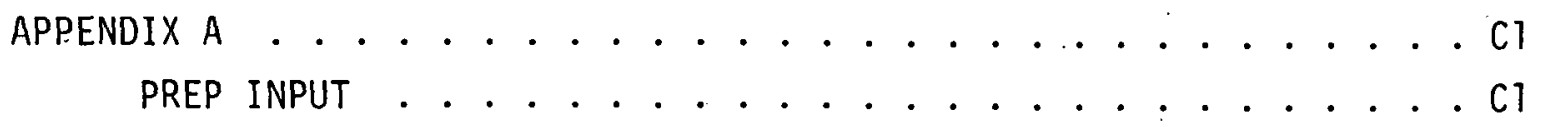

APPENDIX B' . . . . . . . . . . . . . . . . . . . . . . . . . . BT

INPUT FOR KITT-1 . . . . . . . . . . . . . . . . BT

APPENDIX C . . . . . . . . . . . . . . . . . . . . . . . . . . . Cl

INPUT FOR KITT-2 ................... CI

APPENDIX D . . . . . . . . . . . . . . . . . . . . . . . . . . . D1

OUTPUT FROM KITT-1 AND KITT-2.................. . . . . . . . . .

APPENDIX E . . . . . . . . . . . . . . . . . . . . . . . . El

THE USE OF THE PREP AND KITT COMFS FOR NON-TIME-DEPENDENT

PROBLEMS

APPENDIX F . . . . . . . . . . . . . . . . . . . . . . . . . . F1

SAMPLE FAULT TREE EVALUATION . . . . . . . . . . . . . FT

APPENDIX G . . . . . . . . . . . . . . . . . . . . . . . . . GT

REQUIRED JOB CONTROL LANGUAGE. FOR THE EXECUTION OF PREP

AND KITT AT THE NRTS . . . . . . . . . . . . . . . . G GI

FIGURES

(PREP)

1. SAMPLE FAULT TREE . . . . . . . . . . . . . . . . . . . . . . . 3

2. FAULT TREE SYMBOLOGY . . . . . . . . . . . . . . . . . . . 4

3. TREBIL PROGRAM INPUT . . . . . . . . . . . . . . . . . . . . . . . . . . . . .

4. TREBIL PROGRAM OUTPUT . . . . . . . . . . . . . . . . . . . . . . . . 9

5. FAULT TREE CODING FORM. . . . . . . . . . . . . . . . . 28 


\section{FIGURES}

(KITT)

6. A THREE-PHASE COMPONENT . . . . . . . . . . . . . 64

7. THE GENERAL N-PHASE COMPONENT ............. 66

8. MOCK-UP OF A TIME-DEPENDENT FAILURE INTENSITY . . . . . . 67

9. MOCK-UP OF A TIME-DEPENDENT REPAIR INTENSITY . . . . . . . 69

10. THE MULTIPHASE INHIBIT CONDITION .............. 71

11. TRANSLATION OF PHASE INPUT DATA ............. 88

TABLES

(PREP)

1. INFORMATION REQUIRED IN INPUT GROUP $1 \ldots \ldots$

2. RECOMMENDED INPUT VALUES FOR INPUT GROUP 1 FOR A COMBO RUN TO OBTAIN ALL MINIMAL CUT SETS OF ONE AND TWO COMPONENTS . . . . . 22

3. RECOMMENDED INPUT VALUES FOR INPUT GROUP 1 FOR A FATE RUN TO OBTAIN MINIMAL PATH SETS OF THE SYSTEM ......... 23

4. RECOMMENDED INPUT VALUES FOR INPUT GROUP 1 FOR A FATE RUN TO OBTAIN IMPORTANT MINIMAL CUT SETS OF THREE OR MORE COMPONENTS . . 24

5. INPUT GROUP 1 FORMATS . . . . . . . . . . . . . 25

6. INPUT GROUP 2 FORMAT FOR EACH INPUT CARD . . . . . . . . 26

7. INFORMATION REQUIRED IN INPUT GROUP $3 \ldots \ldots$

8. INPUT GROUP 3 FORMAT . . . . . . . . . . . . 30

9. LMDA(I) AND TAU(I) INTERPRETATIONS .............. . . 31

\section{(KITT)}

10. KEY TO "COMPONENT AND INHIBIT INFORMATION" ......... 55

11. KEY TO "MINIMAL SET INFORMATION" IF MINIMAL CUT SETS ARE USED . . 55

12. KEY TO "MINIMAL SET INFORMATION" IF MINIMAL PATH SETS ARÉ USED . 56

13. KEY TO "SYSTEM INFORMATION - UPPER BOUNDS" . . . . . . . 57 


\section{TABLES}

14. KEY TO "DIFFERENTIAL CHARACTERISTICS - BEST BRACKETS" . . . . . 58

15. KEY TO "FAILURE RATE CONTRIBUTIONS" IF MINIMAL CUT SETS ARE USED 58

16. KEY TO "FAILURE RATE CONTRIBUTIONS" IF MINIMAL PATH SETS ARE USED 59

17. KEY TO "DIFFERENTIAL CHARACTERISTICS - LAST BRACKETS" IF MINIMAL CUT SETS ARE USED . . . . . . . . . . . . . . . . . 59

18. KEY TO "DIFFERENTIAL CHARACTERISTICS - LAST BRACKETS" IF MINIMAL PATH SETS ARE USED .................... 60 60

19. KEY TO "INTEGRAL CHARACTERISTICS - BEST BRACKETS" . . . . . . . 60

A-1. INPUT DECK . . . . . . . . . . . . . . . . . A2

A-2. INPUT GROUP 1. . . . . . . . . . . . . . . . . . A3

A-3. INPUT GROUP 2 . . . . . . . . . . . . ........ A4

A-4. INPUT GROUP 3 . . . . . . . . . . . . . . . . . . A5

B-1. INPUT FOR KITT-1 . . . . . . . . . . . . . . . . B2

C-1. INPUT FOR KITT-2 . . . . . . ............... . . 2

D-1. . KEY TO "COMPONENT AND INHIBIT INFORMATION . . . . . . . . . . . D2

D-2. KEY TO "MINIMAL SET INFORMATION" IF MINIMAL CUT SETS ARE USED . . D2

D-3. KEY TO "MINIMAL SET INFORMATION" IF MINIMAL PATH SETS ARE USED - D3 D-4. KEY TO "SYSTEM INFORMATION - UPPER BOUNDS" . . . . . . . . . D4 D-5. KEY TO "DIFFERENTIAL CHARACTERISTICS - BEST BRACKETS" • . . . . D5 D-6. KEY TO "FAILURE RATE CONTRIBUTIONS" IF MINIMAL CUT. SETS ARE USED D5 D-7. KEY TO "FAILURE RATE CONTRIBUTIONS" IF MINIMAL PATH SETS ARE USED D6 -D-8. KEY TO "DIFFERENTIAL CHARACTERISTICS - LAST BRACKETS" :IF .MINIMAL CUT SETS ARE USED . . . .................... . . . . . . .

D-9. KEY TO "DIFFERENTIAL CHARACTERISTICS - LAST BRACKETS" IF MINIMAL PATH SETS ARE USED . . . . . . . . . . . . . . . . . D7

D-10. KEY TO "INTEGRAL CHARACTERISTICS - BEST BRACKETS" . . . . . . . D7 
THE USE OF THE PREP CODES

by

R. E. Narum

IDAHO NUCLEAR CORPORATION

Idaho Fal1s, Idaho 


\section{INTRODUCTION}

The PREP computer program package is designed for use as a preprocessor for the KITT computer programs (Section 2). The PREP programs find the minimal cut sets and/or the minimal path sets from the system's fault tree, and output them in a format compatible for use with KITT.

The programs are written in Fortran IV for the IBM $360 / 75$ computer, and are capable of finding the minimal cut and path sets for fault trees with up to 2000 components and up to 2000 logical gates. The system's minimal cut sets may be obtained by either deterministic testing or Monte Carlo simulation. The system's minimal path sets are found by Monte Carlo simulation.

The input formats have been designed to make the description of the system fault tree to the programs natural for the engineer familiar with standard fault tree terminology. Extensive error checking is done on the input to reduce the chance of obtaining erroneous information from the programs .

\section{Fault Trees}

In order to obtain reliability information about a system from the PREP and KITT programs the defined failed state of the system must first be described in terms of possible combinations of defined component failed states. This is done by means of a schematic device called a fault tree. A fault tree represents a system failed state by means of the logical relationships between the individual component failed states of the system. Figure $l$ is an example of a fault tree ${ }^{[a]}$. The gate labelled "TOP" in [a] A detailed description of the process of drawing a system fault tree can be found in Reference 1 . 
Figure 1 represents the failed state of the system. That is, the TOP event occurs when the system passes from the unfailed to the failed statc. The symbology used to draw fault trees is explained in Figure 2.

\section{Cut Sets}

The fault tree allows the determination of sets of components which when failed will cause the system to be failed. For example, in the fault tree of Figure 1 , if the components 1 and 2 are simultaneously in the failed state the system will be in the failed state. Such a set of components is called a cut set for the system. A cut set is formally defined as any set of system components whinh when simudancouoly failed vuust lile system to be in the failed state.

From the definition of a cut set it can be seen that the set of components $\{4,5,7\}$ represents a cut set of the sample fault tree. Examination of the logical relationships defined by the tree shows that the set of components $\{4,5\}$ also comprises a cut set. The failure of component

- 7 is not necessary to cause the TOP event if components 4 and 5 also fail. On the other hand, neither component 4 nor 5 can caluse system failure by itself". Such a cut sct is called a minimal cut set of the system. A minimal cut set is a smallest set of system components which when failed will cause the system to be failed. A minimal cut set is defined to be in the failed or nonfunctioning state if and only if all of its components are in the failed state, and is defined to. be in the non-failed or functioning slate if and on $\perp y$ if at least one of its componento is in the: functioning state. It can be seen that the system is in the failed state if and only if at least one of its minimal cut sets is in the failed state. Thus, the minimal cut sets represent those sets of components which are critical with regard to system failure. 


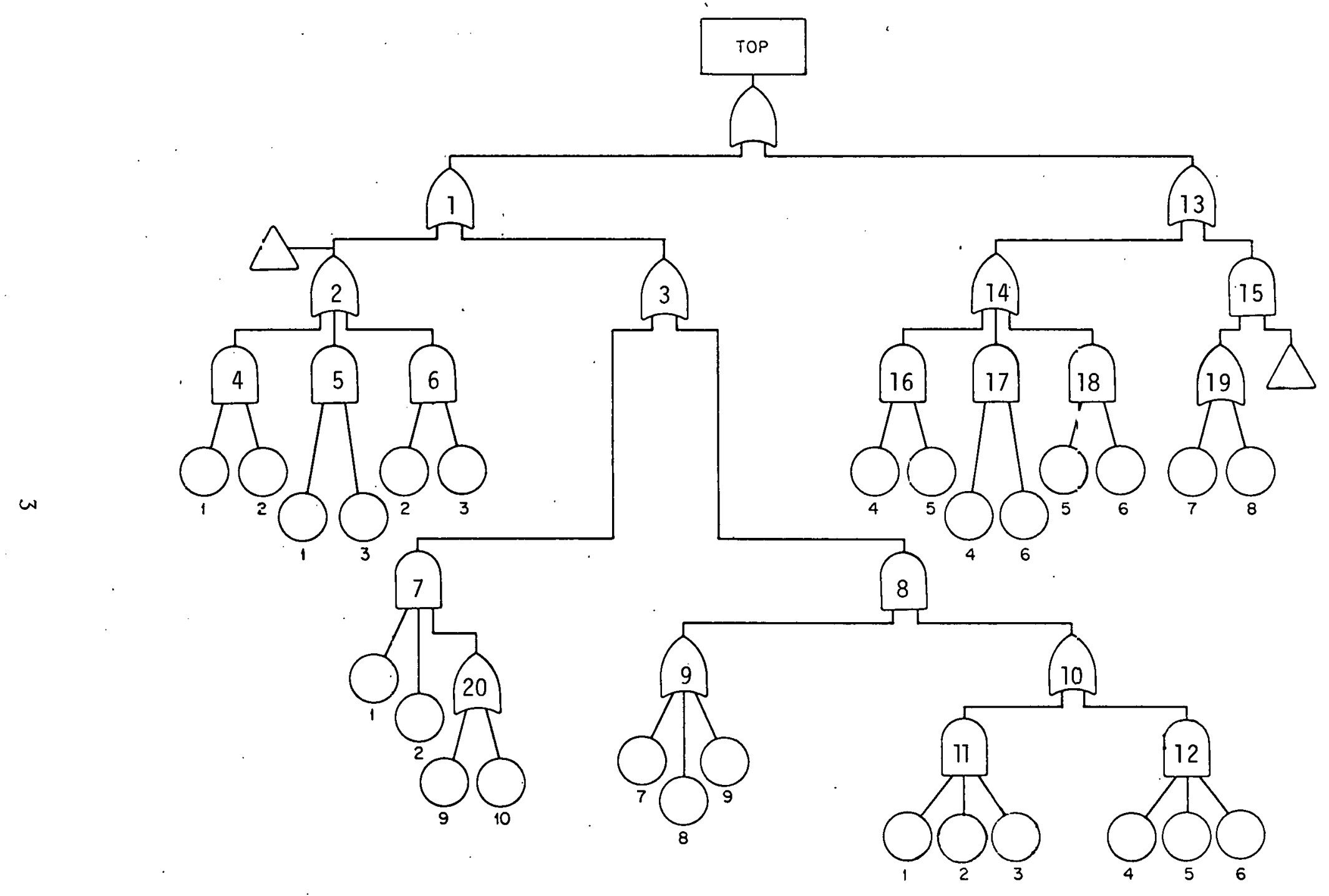

FIGURE 1. Sample Fault Tree 
System component or sasiz fault event.

OR Gate.

This gate is in the zailed state if at least one of its inputs is in the failed state.

Inputs

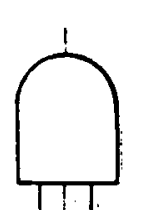

Inputs.

AND Gate.

This gate is in the failed state only if all of its inputs are simaltaneously in their failec states.

Zvent Descriptor.

The rectangle is used to describe the event represented by a gate.

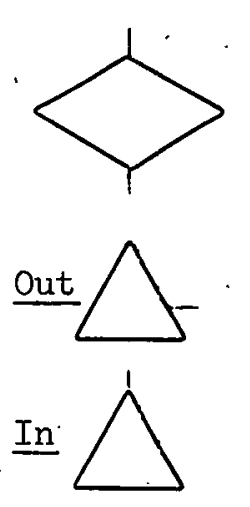

The diamond is used to represent a fault event which is not developed further die to lack of information.

Transfer Symbols.

These symbols are used to transfer an entire part of the tree to other locations on the tree.

\section{Inhibit Gate.}

This represents an event which occurs with some fixed probability of occurrence. The inhibit gate is in the failed state only if its inputs are in the failed state and the inhibit condition has occurred.

The house represents an event which is normally expected to occur. It is treated as a switch on the tree, and is set on or off by the PREP user. 


\section{Path Sets}

It is also possible to use the fault tree to determine sets of com- ponents of a system which must function in order that the system function. For example, in the fault tree of Figure 1, if components 1, 2, 4, and 5 are all functioning simultaneously, then the system will function (cannot be failed). Such a set of components is called a path set for the system. A path set is formally defined as any set of system components which when simultaneously functioning cause the system to function.

From this definition it can be seen that the set of components $\{1$, $2,4,5,6\}$ is also a path set, but that the inclusion of component 6 was unnecessary. On the other hand, no set of three of these components of the system is sufficient to keep the system functioning. Analogeous to the definition of minimal cut set we define a minimal path set of the system as a smallest set of system components which must function in. order for the system to function. A minimal path set is defined to be in the failed state if and only if at least one of its components is in the failed state, and is defined to be in the functioning state if and only if all of its components are in the functioning state. As in the case of minimal cut sets, the state of the system can be determined from the states of the minimal path sets. The system is in the failed state if and only if all of its minimal path sets are in the failed state, and the system is in the functioning state if and only if at least one of its minimal path sets is in the functioning state. Thus, the minimal path sets represent those sets of components which are critical with regard to the system functioning state.

The minimal cut sets and minimal path sets represent two equivalent sources of information about a physical system's state, and knowledge of 
either the minimal cut sets or the minimal path sets is sufficient to obtain the desired system reliability information.

\section{PROGRAM DESCRIPTIONS}

The PREP computer program package is designed to accept an input description of the system fault tree, generate the appropriate logical equivalent, and obtain the system's minimal cut or path sets. These minimal cut or path sets can then be used by the KITT programs to obtain reliability information about the system.

The PREP package is composed of two sections: TREBIL reads the input and generates the logical equivalent of the fault tree and MINSET obtains the minimal cut or path sets of the tree.

\section{TREBIL}

The TREBIL program is designed to accept a description of the system fault tree in a format which is natural for the engineer, and to generate a logical equivalent of that fault tree'.

'l'he fault tree logic building algorithm requires that each logical gate and each component of the tree be given an alphanumeric name. This name can be any arbitrary combination of from one to eight characters. Figure 3 is an example of the input of a fault tree to the TREBIL program. One card is read in for each logical gate in the fault tree. The card contains the name of the gate, the type of the gate ("AND" or "OR"), the number and names of the other gates which are attached to it, and the number and names of components which are attached to it.

Since TREBIL is designed to accept only "AND" gates and "OR" gates as input, other types of gate logic must be described in terms of these simple gate types. If such a description is not possible in TREBIL, it 


\section{TREBIL PROGRAM INPUT}

\begin{tabular}{|c|c|c|c|c|c|c|c|c|}
\hline \multicolumn{9}{|c|}{ SPERT TREE } \\
\hline Al & $O R$ & 30 & $\mathrm{~A} 2$ & A3 & A4 & & & \\
\hline$A 2$ & OF & 40 & Al1 & A 12 & A13 & A 14 & & \\
\hline A3 & OR & 60 & $A 5$ & $A 6$ & A7 & $A B$ & A9 & A10 \\
\hline A4 & AND & 40 & Al1 & A12 & A13 & A 14 & & \\
\hline$A 5$ & AND & 20 & A11 & A 12 & & & & \\
\hline$A 6$ & AND. & 20 & A11 & A 13 & & & & \\
\hline A7 & AND & 20 & Al1 & A 14 & & & & \\
\hline$A B$ & AND & 20 & $A 12$ & $A 13$ & & &. & \\
\hline A9 & AND & 20 & A 12 & A 14 & & & & \\
\hline A10 & AND & 20 & A13 & A 14 & & & & \\
\hline A11 & AND & 20 & $A 15$ & A19 & & & & \\
\hline A15 & OR & 30 & A 17 & A18 & & & & \\
\hline A16 & OR & 13 & A44 & $\mathrm{K} 349 \mathrm{GHI}$ & $K 3491$ & K349SEC & & \\
\hline A17 & AND & 03 & WM50111G & RBCKTSP & MAGIHOLO & & & \\
\hline A18 & OR & 16 & B1 & W52371P & $\$ 52011 P$ & $1451501 P$ & $\$ 50141 P$ & $W 52841 P$ \\
\hline B1 & OR & 07 & $H 32121 P$ & T22861P & C951A1P & C828F1P & C728FIP & C528F1P \\
\hline A1 2 & AND & 20 & A114 & A26 & & & & \\
\hline A23 & OR & 13 & A45 & K3511 & $\mathrm{K} 351 \mathrm{GH} 1$ & K351SEL & & \\
\hline A24 & AND & 03 & WM50211G & RECKTSP & MAG2HOLD & & & \\
\hline A 2.5 & OR & 16 & B3 & T22961P & C952A1P & C829F1P & C729F.IP & C629F1P \\
\hline 83 & OR & 07 & W52391P & $W 52081 P$ & $W 51591 P$ & W50231P & W52931P & H4025.1P \\
\hline A19 & OR & 30 & A20 & A21 & A22 & & & \\
\hline $\mathrm{A} 20$ & OR & 13 & A44 & K349JK1 & K3491 & K349SEC & & \\
\hline
\end{tabular}

FIGURE 3. 
may be possible to mock up the true system behavior in the KITT programs. The one exception is the "inhibit" gate. The "inhibit" gate is input as an "AND" gate with the inhibit condition attached as a component.

The logical equivalent of the fault tree is in the form of a FORTRAN subroutine. The subroutine is compiled as an intermediate job step under OS/360, and link-edited together with the routines in MINSET. Figure 4 is an example of the logical subroutine TREE generated by TREBIL. Each component is internally indexed by TREBIL, and is treated as an element in a FORTRAN logical array (called. "X"). Similarly, each gate ié indcucd and treated as an element in another FORTRAN logical array (called "A"). In addition to building TREE, TREBIL serves as the input processor for the MINSET program. All the data required by MINSET are read in, and checked for errors, by TREBIL.

\section{MINSET}

The MINSET program determines the minimal cut or path sets of the fault tree. The MINSET program allows minimal cut sets to be found by either deterministic testing or by Monte Carlo simulation.. Minimal path sets. must be found by Monte Carlo simulation.

Deterministic Testing (COMBO)

COMBO is the deterministic testing algorithm for obtaining minimal cut sets. In COMBO each component is first failed individually to obtain the one component minimal cut sets. Next, every possible combination of two components is failed to obtain the two component minumal cut: sets. (The two component cut sets which contain a one component minimal cut set are rejected in this procedure.) The algorithm proceeds in this manner until minimal cut sets are obtained having $\mathrm{n}$ components where $\mathrm{n}$ is specified by the user. The algorithm may also be started at minimal cut sets having 


\section{TREBIL PROGRAM OUTPUT}

\section{SPERT TREE}

THIS IS THE SUBROUTINE GENERATED BY TREBIL SUBROUTINE TREE LOGICAL TOP, A(2000), X(2000) COMMON/TREES/A, $X$, TOP

$A(\quad 1)=X(1) \cdot O R \cdot X(\quad 2) \cdot O R \cdot X(\quad 3)$

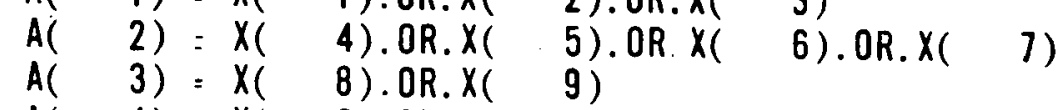

$A(4) \cdot X(10) \cdot 0 R \cdot X(11) .0 R \cdot X(12)$

$A(5)=X(13) \cdot$ OR.X( 14).OR.X( 15).OR.X( 16)

* OR. $X(17)$

$A(\quad 6) \cdot X(11) \cdot O R \cdot X(12)$

$A(\quad 7)=X(18)$. OR.X( 19).OR.X( 20).OR.X( 21)

* $\quad$ OR.X( 22)

$A(\quad 8)=X(23) \cdot O R \cdot X(24)$

$A(\quad 9)=X(25)$. OR.X( 26)

$A(10)=X(27) . O R \cdot X(28) . O R \cdot X(29) . O R \cdot X(30)$

* .OR.X( 31).OR.X( 32).OR.X( 33)

$\left.A(11)=X(34) . O R \cdot X(10) . O R \cdot X(11) . O R \cdot \dot{X}_{(}^{\prime} \quad 12\right)$

$A(\quad 12)=X(35) . O R \cdot X(36) . O R \cdot X(37) . O R \cdot X(38)$

$A(13)=X(40) .0 R \cdot X(41)$

FITSURE 4. 
other than one component (for example, searching first for two component minimal cut sets and ending at n component minimal cut sets).

Deterministic testing is the most reliable method for obtaining minimal cut sets since it is theoretically possible to test all possible combinations of components. In a fault tree with a large number of components (eg, having more than 100 components) this is not possible in any reasonable amount of computer time. Thus, in a large fault tree, deterministic testing should only be used to obtain minimal cut sets of one, two, and possibly three components. This apparent restriction is not really detrimental since the COMBO algorithm has been programmed so that the minimal cut jets are found in order of number of components. In most cases, the number of components in each minimal cut set is the factor which determines its relative importance. For example, if each component in the fault tree has a failure intensity $\lambda$, then the probability of a failure of cach one component minimal cut set is proportional to $\lambda$ whereas the probabilitity of $a$ failure of each $\mathrm{n}$ component minimal cut set is proportional to $\lambda^{\mathrm{n}}$. Since most components have failure intensities below $10^{-3}$, it wolld take well over $10^{3}$ two component minimal cut sets to equal the contribution of a single one component minimal cut set to the probability of failure of the system.

In order to use COMBO, the starting (MIN) and ending (MAX) values for the number of components in the minimal cut sets found must be specified. If the minimum number specified is o (or left blank), then Monte Carlo simulation is used. The maximum number of components allowcd by the program is 10 (MAX $\leq 10)$. 


\section{Monte Carlo Simulation (FATE)}

The use of a Monte Carlo simulation procedure to obtain the minimal cut or path sets of a fault tree is fairly efficient since the information obtained is qualitative, rather than quantitative; ie, only the components in a minimal' cut or path set are determined. In FATE, the subroutine containing the simulation procedure, all the components are assumed nonrepairable and independent. (COMBO also assumes independence of the components.) The nonrepairability assumption, of course, in no way affects the validity of the minimal cut or path sets obtained.

In the Monte Carlo procedure, failures of the components are chosen according to their failure distributions and TREE tested for a system failure. If a failure has occurred, then the set of components, which are failed, is sorted to obtain the minimal cut set. Once the minimal cut set has been found, it is checked against all previously found minimal cut sets to eliminate duplicates. This procedure is called a Monte Carlo trial.

An input switch (IDEXI) is provided which causes the program to search for a minimal path set on any trial which does not result in system failure.

The probability of a failure before time $t$ for a component is computed by the exponential distribution. If $P(T)$ is this probability of a failure before time $\mathrm{T}$, then

$$
P(T)=1-\exp (-\lambda T)
$$

where $\lambda$ is the failure intensity (per hour) for the particular component and $t$ is the time in hours. From the above equation, it can be seen that $\mathrm{P}(\mathrm{T}=0)=0$, and that $\operatorname{limP}(\mathrm{T})=1$. That is, all components are assumed $t \rightarrow \infty$ initially functioning, and eventually, must all fail. The times of failures are chosen from the exponential distribution based on an input value for $T$. 
This input time $T$ is usually called a "mission length". In FATE, however, T has no connection with any "mission" of the system. In fact, it will be called a "mixing parameter" in this report since, as will be shown, it allows the user to exercise some measure of control over the way in which FATE finds the minimal cut sets. A time of failure $t$ is computed for each component such that $t$ is always less than $T$. This time of failure $t$ is from the distribution:

$$
a(t)=\frac{\lambda e^{-\lambda t}}{1-e^{\lambda T}}
$$

where $a(t)$ is the probability distribution for $t$.

Unce times of f'ailure $t$ have been computed for each component, the component which has the smallest time of failure is first failed, and TREE is called. If the system is not in the failed state, then the component with the next smallest time of failure is failed, and TREE is tested again. This process is repeated until the system fails. When system failure occurs, this set of failed componenta is redused to the minimal cut set.

If PATE is used wo obtuin the system's minimal path sets, then at each trial, the entire set of components is first failed so that the system will be in the failed state. Components are then selected one at a time from the uniform distribution and put in the nonfailed state. After each such selection of a component TREE is called, and the system is checked to determine if it has changed from the failed to the nonfalled state. When the system is in the nonfailed state the set of selected components comprise a path set of the system. This path set is then reduced to a minimal path set.

If inhibit conditions are present in the fault tree, then they will be printed as members of the minimal cut sets or path sets, when their 
existence or nonexistence is necessary along with the components in that set. The KITT codes automatically handle these inhibit conditions in the proper manner.

Switches are controlled by the user. A switch is either always failed or nonfailed and is never printed out as a member of a minimal cut or path set.

In order to make effective use of the PREP program pakage, some care must be taken in the choice of the mixing parameter $T$. The correct choice may depend upon the particular fault tree, but there are some general guide lines which can be followed.

Since FATE finds the minimal cut sets of the system by failing components according to times of failure chosen from a(t), the value of the mixing parameter used by FATE determines the order in which the components fail. Thus, varying $T$ will vary the manner and order in which the minimal cut sets are found. To see this, consider $q(t)$;

$$
q(t)=\frac{e^{-\lambda t} \lambda}{1-e^{-\lambda T}} .
$$

If $T$ is chosen very large so that $e^{-\lambda T}$ approaches zero, then $q(t)$ approaches the true exponential distribution:

$$
a(t)=e^{-\lambda t} \lambda
$$

In this case, the computed times of component failure will approximate the true times of failure (assuming nonrepairability).

If on the other hand, $T$ is so small such that $\lambda T<<1$ for each component $\lambda$, then न.(t.) a.pnrnximates a uniform distribution. That is, the computed times of failure will be uniformly distributed in the interval $[0, T]$. 
To verify this, consider:

$$
e^{-\lambda T}=\sum_{n=0}^{\infty}(-1)^{n} \frac{(\lambda T)^{n}}{n !} .
$$

Thus, for $\lambda T<<I$ orders of $\mathrm{n}$ greater than one may be neglected and

$$
e^{-\lambda T} \approx 1-\lambda T
$$

Thus a(t) approaches $\frac{1}{T}$.

It can he spen that., if $T$ is darge [Equation (4)] the tiules of fullures will be well structured. That is, those components which have large failure intensities $(\lambda)$ will have small times of failure while those with small failure intensities $(\lambda)$ will have large times of failure. This means that FATE will first find those minimal cut sets which are most important to system failure and then will find the lesser important minimal cut sets. If on the other hand, $T$ is chosen so that $\lambda T<<1$; then the failure times generated will tend to be uniformly mixed. This means that FATE would find the minimal cut sets in a random manner.

If minimal path sets are found by FAIE, then they are always found in a random manner as described previously. 


\section{INPUT DESCRIPTION AND RECOMMENDATIONS}

\section{Input Deck Description}

The input to TREBIL consists of the three input groups:

1. Control information

2. The Fault Tree description

3. The failure and repair data for the components.

The beginning of each group of data is indicated by a special control card of the form:

$$
\begin{array}{lll}
\text { Col. } 1 & \text { Col. } 2 & \text { Col. } 3 \\
* & \text { blank } & \text { KEYWORD }
\end{array}
$$

with KEYWORD replaced by DATA for input group I, TREE for input group 2, and RATES for input group 3.

The end of each input group is indicated by a card with the word END beginning in card column 1 .

Each of these input groups may be preceded by any number of comments cards. The first such card appearing in the input deck is used as a title card and is printed on each page of output.

The total input deck has the following format:

1. TITLE CARD

2. \{COMMENTS CARDS

3. *bDATA ( $b=$ blank column)

4. \{CONTROL PARAMETERS

5. END

6. \{COMMENTS CARDS

7. *bTREE

8. \{FAULT TREE DESCRIPTION\}

9. END

10. \{COMMENTS CARDS

11. *bRATES

12. \{FAILURE AND REPAIR DATA

13. END 


\section{Input Group 1}

Input Group I consists of the control parameters and switches that determine the flow through MINSET, and determine the type of output obtained. Table 1 contains a brief description of the contents of Input Group 1.

Information required in Input Group I

TABLE 1 .

\begin{tabular}{|c|c|}
\hline $\begin{array}{l}\text { FORTRAN } \\
\text { VARIABLE }\end{array}$ & DE'SCRIPTION \\
\hline 1. NG & Number of gates in the fault tree \\
\hline 2. $M I N$ & $\begin{array}{l}\text { Minimum size of the minimal cut sets to be found } \\
\text { by deterministic testing (COMBO) }\end{array}$ \\
\hline 3. MAX & $\begin{array}{l}\text { Maximum size of the minimal cut sets lo be found } \\
\text { by deterministic testing (COMBO) }\end{array}$ \\
\hline 4. MCS & $\begin{array}{l}\text { Minimum oizc of the minimal cut sets to be stored } \\
\text { by Monte Carlo (FATE) }\end{array}$ \\
\hline 5. NREJEC & Random number starter \\
\hline 6. IN' $\mathrm{I}$ & Number of Monte Carlo trials to run \\
\hline 7. IREN & Monte Carlo mixing parameter switch \\
\hline 8. TAA & Monte Carlo mixing parameter \\
\hline 9. IDEXI & $\begin{array}{l}\text { Switch which determines if minimal cut sets or } \\
\text { minimal path sets are to be obtained from FATE }\end{array}$ \\
\hline 10. IDEX2 & $\begin{array}{l}\text { Switch which determines whether minimal sets are } \\
\text { to be printed or punched, or both }\end{array}$ \\
\hline
\end{tabular}




\section{Recommendations- for Input Group 1}

Input Parameters Which Control Deterministic Testing

This set of input parameters is used by the deterministic testing routine (COMBO) only. They should be left blank if Monte Carlo simulation (FATE) is used.

MIN

The value of MIN is used by the deterministic testing routine COMBO and is the minimum size of the minimal cut sets that are to be obtained. If MIN $=0$, then the Monte Carlo process is to be used. The value of MIN can be no greater than 10.

MAX

The variable MAX stops the deterministic testing process. The maximum value which MAX may be given is 10 . The value of MAX is the maximum size of the minimal cut sets to be found by COMBO. If MIN > MAX, an error message is written and problem execution terminated.

The size of the system being tested must be considered when choosing values for MIN and MAX. A reasonably accurate rule is to allow about $10^{-5}$ minutes of IBM $360 / 75$ time per combination. If the system fault tree has m distinct components, and all combinations of $\mathrm{n}$ components are to be tested, then this will require $\left(\frac{m}{n}\right) 10^{-5}$ minutes on the IBM $360 / 75[a]$. For example, if $\mathrm{m}=500$, and we wish to find all 2-component minimal cut sets $(\mathrm{n}=$ MIN $=$ MAX $=2)$, then this will require about 1 minute of time in COMBO. If we let $\mathrm{n}=3$, then the time required is about $10^{3}$ minutes. It is recommended that every system be run using COMBO to find the minimal cut sets consisting of one component, and that most systems be

[a] $\left(\begin{array}{c}m \\ n\end{array}\right)$ is the binomial coefficient, $\left(\begin{array}{l}m \\ n\end{array}\right)=\frac{m !}{n !(m-n) !}$. 
run to find the minimal cut sets consisting of two components. If larger minimal cut sets are desired from the system, then the number of components and number of combinations must be considered before deciding to use deterministic testing rather than the Monte Carlo algorithm.

\section{Input Parameters Which Control Monte Carlo Simulation}

This set of input parameters is used by the Monte Carlo testing routine (FATE) only. They should be left blank if deterministic testing (COMBO) is used.

MCS

The value of MCS is the size of the smallest minimal cut sets that the analyst wishes to obtain using FaTF (Monte Corlo). If, PuI Exaul, te, a run has been made using COMBO to find the minimal cut sets with one and two components, then MCS $=3$ should be used if a subsequent run is made using FATE. If MCS $=0$ or is left blank, then MCS $=1$ is assumed.

\section{NREJEC}

The random number starter NREJEC is an integer which is used to reject the first NREJEC random numbers found by the random numbers generator in FATE. Its value is arbitrary, but it must be the same to reproduce the results of a previous run. If COMBO is being used, then NREJEC need not be supplied.

\section{TAA}

The Monte Carlo mixing parameter T (FORTRAN mnemonic is TAA) determines whether FATE mixes component failures uniformly or keeps them structured with regard to their importance. If $T$ is such that $\lambda T<<I$ for all values of the component failure intensities, $\lambda$, appearing in the 
system, then the component failures will be mixed uniformly. If $\lambda \mathrm{T}$ is large, say on the order of 10 or 100 , then the failures will occur in a manner which preserves the relative importance of the components and, hence, the importance of the minimal cut sets.

Let $\lambda_{\min }$ be the smallest failure intensity for all components in the system, and let $\lambda_{\max }$ be the largest failure intensity for all components in the system. If uniform mixing is desired, then it is recommended that $T$ be chosen so that $\lambda_{\max } T<.01$. Then $\lambda T<.01$ for all values of $\lambda$ for components in the fault tree, and the cut sets will be randomly obtained. If the relative importance of the minimal cut sets is of concern, then it is recommended that $\mathrm{T}$ be chosen so that $\lambda_{\min } \mathrm{T}>10$. Then $\lambda \mathrm{T}>10$ for all $\lambda$.

If there is a maximum time of interest $T_{O}$, then choosing $T=T_{0}$ will cause FATE to find those minimal cut sets first which are most likely to fail up to time $\mathbb{T}_{0}$.

If minimal path sets are to be obtained then, since uniform mixing is always used, T need not be input to FATE.

IREN

The integer switch IREN is used to cause FATE to choose a value for the mixing parameter $T(T A A)$. If IREN $=I$ then $T=.01 / \lambda_{\max }$ so that $\lambda T \ll 1$ for all values of $\lambda$. If IREN $=2$ then $T=10.0 / \lambda_{\min }$ so that $\lambda T>10$ for all values of $\lambda$. In both of these cases any input value for $T$ will be ignored. If IREN $=3$ then a value for $T$ will be reaa from the

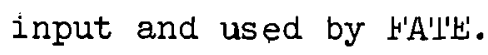


It is recommended that IREN $=1$ or $I R E N=2$ be used whenever possible to reduce input errors (see discussion of TAA).

If minimal path sets are to be obtained then IREN may be left blank.

NTR

If COMBO has been used to obtain all one and two component minimal cut sets, and if one component minimal cuts are found, FATE need not be run. In this case, NTR is not applicable (ie, is left blank).

If FATE is to be used to find minimal cut sets (for example, if no one component cut sets were found by COMBO) then one of two procedures may be followed.

One procedure is to make a single FATE run with NTR $=5000$. In this case, it is recommended that IREN $=2$ (that is the cut sets are structured when found).

$\Lambda$ better procedure is to make one run of 1000 trials (that is NTR $=$ 1000) with IREN $=2$. If minimal cut sets containing MCS and MCS +1 com. ponents have been found, then these are the most important minimal cut sets, and no further runs neen to be made.

If minimal cut sets of MCS and MCS +1 components are not found, then another run should be made with NTR $=5000$ and IREN $=1$ (uniform mixing). l'his will find other minimal cut sets to be added to those found in the first run.

The resulting cut sets from these two runs are to be combined and duplicate minimal cut sets eliminated.

If FATE is being used to obtain minimal. path sets for the system, then NTR $=5000$ is recommended. The program will store no more than 500 minimal path sets, and no more than 25 components in any path set. 
IDEX1

The switch IDEXI determines whether minimal cut sets or minimal path sets are found by FATE. If IDEXI $=0$, then minimal cut sets are obtained. If IDEX $=1$, then minimal path sets are obtained.

Input Parameters Which Are Required for Both Deterministic Testing and Monte Carlo Simulation

IDEX2

The switch IDEX2 determines whether the minimal cut and path sets obtained by COMBO and/or FATE are printed, punched, or both printed and punched. If IDEX2 $=0$, then the minimal cut or path sets are printed only. If IDEX2 $=1$, then the minimal cut or path sets are both printed and punched.

The recommended input values for Input Group 1 are summarized in Tables 2, 3, and 4 . 
TABLE 2.

Recommended Input Values for Input Group 1

for a COMBO Run to Obta in A1.1 Minimal Cut Sets

$$
\text { of One and Two Components. }
$$

\begin{tabular}{|c|c|c|}
\hline $\begin{array}{l}\text { Fortran } \\
\text { Variable }\end{array}$ & Value. & Description \\
\hline 1. NG & Variable & $\begin{array}{l}\text { Number of logical gates in the } \\
\text { fault tree }\end{array}$ \\
\hline 2. MIN & 1 & $\begin{array}{l}\text { Smallest combinations to be } \\
\text { considered }\end{array}$ \\
\hline 3. MAX & 2 & $\begin{array}{l}\text { Largest combinations to be } \\
\text { considered }\end{array}$ \\
\hline 4. MCS & Not Applicable & \\
\hline 5. NREJEC & Not Applicable & \\
\hline 6. NTSS & Not Applicalle & \\
\hline 7. IREN & Not_Appl1 sab7e. & \\
\hline 8. TAA & Not Applicable & \\
\hline 9.. IDEXI & Not Applicable & . \\
\hline 10. IDEX2 & $\begin{array}{l}0 \\
1\end{array}$ & $\begin{array}{l}\text { If cut sets are to be printed } \\
\text { only } \\
\text { If cut sets are to be both } \\
\text { printed and punched }\end{array}$ \\
\hline
\end{tabular}


TABLE 3 .

Recommended Input Values for Input Group 1 for a FATE Run to Obtain Minimal Path Sets of

The System

\begin{tabular}{|c|c|c|}
\hline $\begin{array}{l}\text { Fortran } \\
\text { Variable }\end{array}$ & Value & Description \\
\hline 1. NG & Variable & $\begin{array}{l}\text { Number of logical gates in the } \\
\text { fault tree }\end{array}$ \\
\hline 2. $M I N$ & o or left blank & Indicates FATE is to be run \\
\hline 3. MAX & Not Applicable & \\
\hline 4. MCS & 0 or 1 & $\begin{array}{l}\text { Indicates all sets are to be } \\
\text { stored }\end{array}$ \\
\hline 5. NREJEC & 500 & $\begin{array}{l}\text { The first } 500 \text { random numbers } \\
\text { will be rejected }\end{array}$ \\
\hline 6. NTR & 5000 & $\begin{array}{l}5000 \text { Monte Carlo trials will } \\
\text { be run }\end{array}$ \\
\hline 7. IREN & Not Applicable & \\
\hline 8. TAA & Not Applicable & $\therefore$ \\
\hline 9. IDEXI & 1 & $\begin{array}{l}\text { Causes FATE to find minimal } \\
\text { path sets }\end{array}$ \\
\hline 10. IDEX2 & 0 & $\begin{array}{l}\text { If minimal path sets are to be } \\
\text { printed only }\end{array}$ \\
\hline & 1 & $\begin{array}{l}\text { If minimal path sets are to be } \\
\text { both printed and punched }\end{array}$ \\
\hline
\end{tabular}


TABLE 4.

Recommended Input Values for Input Group 1

for a FATE Run to Obta in Important Minimal Cut Sets

of Three or More Components

\begin{tabular}{|c|c|c|}
\hline $\begin{array}{l}\text { Fortran } \\
\text { Variable }\end{array}$ & Value & Description \\
\hline 1. NG & Variable & Number of gates in the fault tree \\
\hline 2. $M I N$ & 0 or left blank & \\
\hline 3. $\mathrm{MAX}$ & Not Applicable & \\
\hline 4. MCS & 3 & $\begin{array}{l}\text { Indicates that only minimal cut } \\
\text { sets with } 3 \text { or more components } \\
\text { are to be stored in FATE }\end{array}$ \\
\hline 5. NREJEC & 500 & $\begin{array}{l}\text { The first } 500 \text { random numbers } \\
\text { will be rejected } \\
\text {. }\end{array}$ \\
\hline 6. NTR & 1000 & $\begin{array}{l}1000 \text { Monte Carlo trials will be } \\
\text { run }\end{array}$ \\
\hline 7. IRRN & 2 & $\begin{array}{l}\text { Causes FATE to choose the mixing } \\
\text { parameter } T \text { such that } \lambda_{\min }>10 .\end{array}$ \\
\hline 8. TAA & blank & \\
\hline 9. IDEXI & .0 & $\begin{array}{l}\text { Causes FATE to find minimal cut } \\
\text { sets }\end{array}$ \\
\hline 10. IDEX2 & 0 & $\begin{array}{l}\text { If minimal cut sets are to be } \\
\text { printed only } \\
\text { If cut sets are to be both } \\
\text { yrinled and punched }\end{array}$ \\
\hline
\end{tabular}


TABLE 5.

Input Group 1 Formats

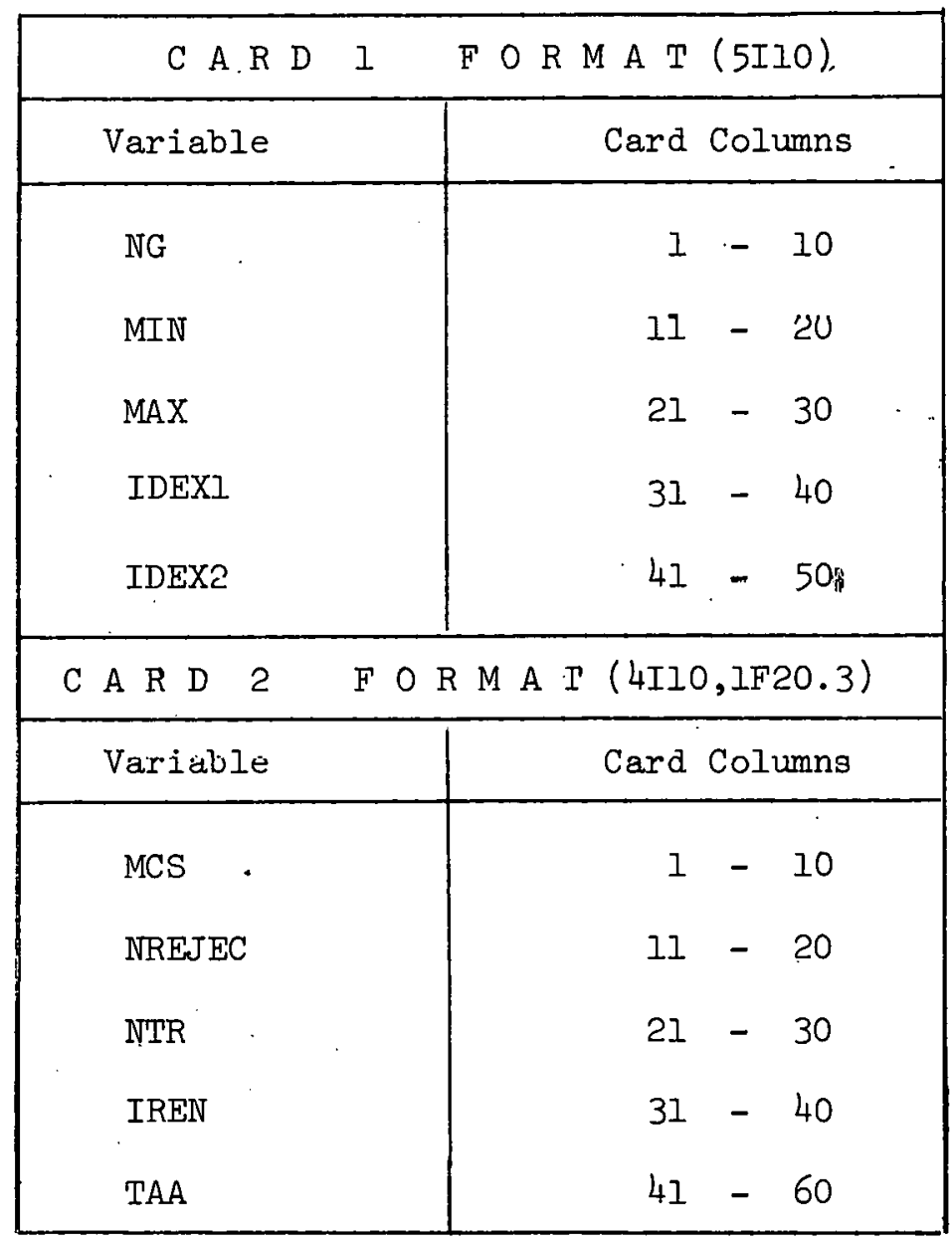




\section{Input Group I Formats}

The variables for Input Group 1 are input on two cards. The card formats are described in Table 5.

\section{Input Group 2}

Information required for Input Group 2 is the name of each gate in the fault tree, its type, the number of inputs to it, and the names of its inputs. The information is supplied one gate per card. The input format tor Input Group 2 is given in Table 6 .

TABLE 6 .

Input Group 2 Format for Each Input Card

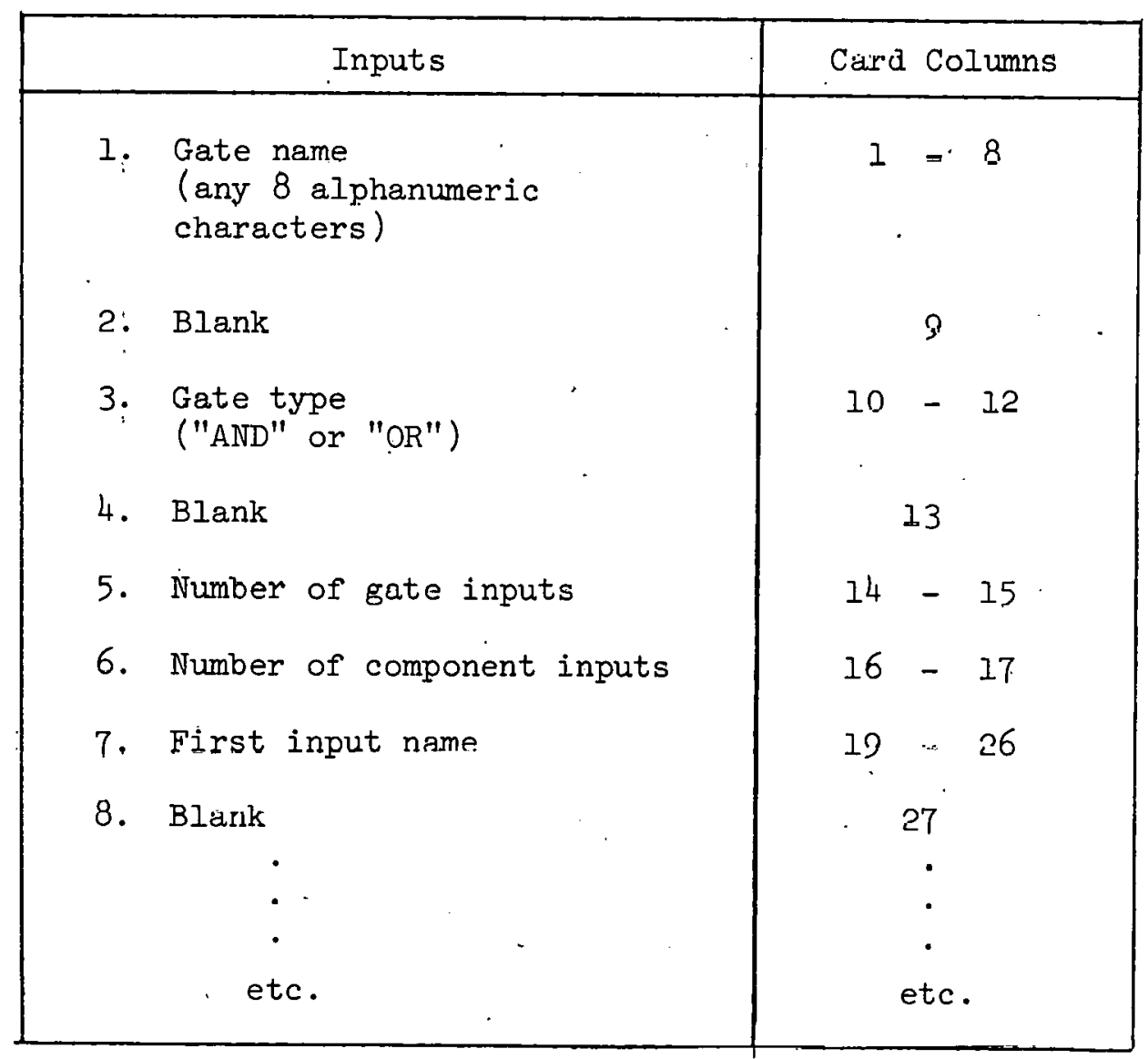


All of the inputs described in Table 6 must begin in the left most of the columns indicated for each of them except for Inputs 5 and 6 which must be right justified in the field.

All gates which input a described gate must be listed before any components which input that gate.

All inhibit conditions and switches are input in Group 2 in exactly the same manner as components are input.

In order to facilitate describing the fault tree to TREBIL, a special input form has been designed with the appropriate columns ruled off and labelled. Figure 5 is an example of the use of the input form.

\section{Recommendations for Input Group 2}

The cards in Input Group 2 may be supplied in any order. The TREBIL algorithm will determine the necessary logical sequence.

A naming convention should be established for the fault tree so that each gate and component can be identified with its location on the tree. A convention will aid in debugging the input, and aid in the interpretation of program output. An example of a convention which works quite well in this regard is to incorporate a numbering scheme into the gate and component names. The examples in Figure 3 illustrate this convention.

\section{Input Group 3}

Information required for Input Group 3 are a failure intensity for each component, repair data for each component, inhibit failure probabilities, and switch settings (on or off). 


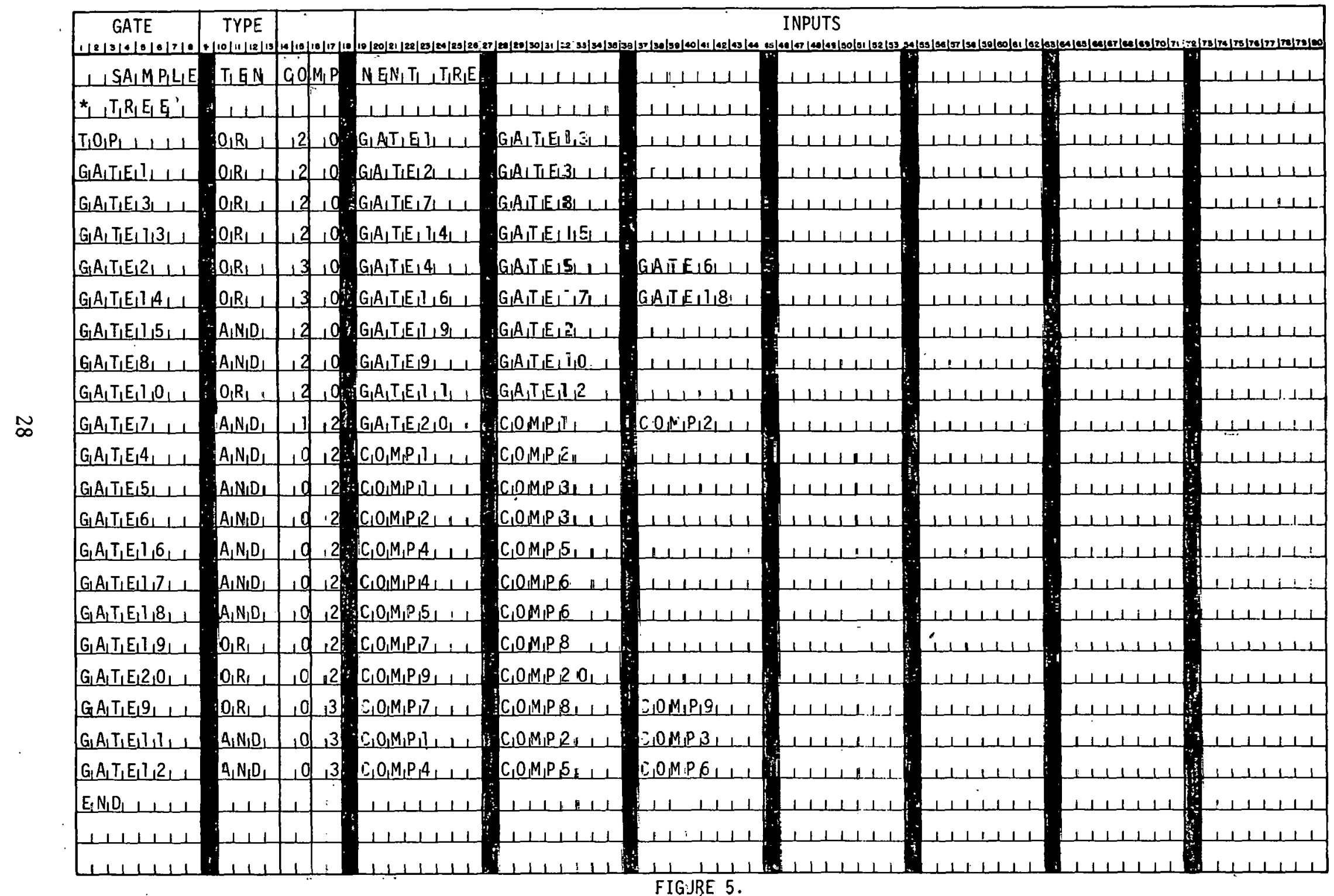




\section{Description and Recommendations for Input Group 3}

The component name $\underline{\text { NAM(I) }}$ must be the same alphanumeric name used in Input Group 2.

The constant failure intensity LMDA(I) is used in FATE to determine time of failure. The failure intensity must be given per $10^{6}$ hours. For example, a failure intensity of $3.6 \times 10^{-6} / \mathrm{hr}$ would be entered on the data card as 3.6. If the system does not have a constant failure intensity for each component (eg, when a multiphase system is to be run) then an average value for the component failure intensity must be used.

The constant repair time TAU(I) is not used by the PREP preprocessor unless the component is an inhibit condition or a switch. The values input are, however, punched in the appropriate format for possible use in KITT. If a component is nonrepairable, or does not have constant repair time, then TAU(I) is to be punched as 0.0 or left blank.

If the $i^{\text {th }}$ component is actually an inhibit condition or a switch, then this is indicated by punching LMDA(I) as a zero or a negative number. If LMDA(I) has been punched 0.0 or a negative number, then TAU(I) indicates whether the component is a true inhibit condition or a switch. If $\operatorname{TAU}(I)$ is greater than 0.0 , but less than 1.0 , then component $I$ is a true inhibit condition and $\mathrm{TAU}(I)$ is its failure probability. If TAU(I) is equal to 0.0 , then component $I$ is a switch, and is never in the failed state ("OFF"). If TAU(I) is equal to 1.0 , then component I is a switch which is always failed ("ON"). These options are outlined in Table 9.

It is noted from Table 8 that the input quantities in Group 3 are supplied two per card. 
TABLE 7.

Information Required in Input Group 3

\begin{tabular}{|c|c|}
\hline Fortran Variable & Description \\
\hline $\operatorname{NAM}(I)$ & $I^{\text {th }}$ component name \\
\hline $\operatorname{LAMDA}(I)$ & $\begin{array}{l}\text { I }^{\text {th }} \text { component failure intensity } \\
\text { (per } 10^{6} \text { hour) or inhibit, switch } \\
\text { indicator }\end{array}$ \\
\hline TAU(I) & $\begin{array}{l}\text { Ith component repair time or inhibit } \\
\text { failure probability }\end{array}$ \\
\hline
\end{tabular}

TABLE 8.

Format $(2(A 8,2 X, F 10.6, F 10.3,7 X))$

Input Group 3 Format

\begin{tabular}{|l|c|}
\hline Input Quantity & Card Columns \\
\hline $\operatorname{NAM}(I)$ & $1-8$ \\
blank & $9-10$ \\
$\operatorname{IMMA}(\mathrm{I})$ & $11-20$ \\
$\operatorname{TAU}(\mathrm{I})$ & $21-30$ \\
$\operatorname{blank}$ & 31 \\
$\operatorname{NAM}(\mathrm{J})$ & $32-39$ \\
$\operatorname{blank}$ & $40-41$ \\
$\operatorname{LMDA}(\mathrm{J})$ & $42-51$ \\
$\operatorname{TAU}(\mathrm{J})$ & $52-61$. \\
\hline
\end{tabular}


TABLE 9.

LMDA(I) and TAU(I) Interpretations

\begin{tabular}{|l|l|l|}
\hline LDMA (I) & \multicolumn{1}{|c|}{ TAU(I) } & \multicolumn{1}{c|}{ Interpretation } \\
\hline Positive & positive & $\begin{array}{l}\text { component constant } \\
\text { repair time }\end{array}$ \\
\cline { 2 - 3 } & equal to 0.0 or blank & $\begin{array}{l}\text { component is nonrepair- } \\
\text { able or does not have a } \\
\text { constant repair time }\end{array}$ \\
\hline \hline Negative or 0.0 & equal to 0.0 & $\begin{array}{l}\text { component is a switch } \\
\text { and never fails (is off) }\end{array}$ \\
\hline & $\begin{array}{l}\text { component is a switch } \\
\text { that is always failed } \\
\text { (is on) }\end{array}$ \\
\hline & greater than 0.0 but & $\begin{array}{l}\text { component is a true in- } \\
\text { hibit condition, and } \\
\text { TAU(I) is its probabi- } \\
\text { lity of occurrence }\end{array}$ \\
\hline
\end{tabular}




\section{OUTPUT TROM PREP}

The output from PREP is in two distinct sections. The first section is the output from TREBIL; the second section is the output from MINSLI. These two sections are physically separated by computer system messages and a job step in which the subroutine TREE is compiled for use by MINSET.

\section{Output From TREBIL}

Output from the TREBIL program consists of a printout of the input, the subroutine TREE, and cross-reference Tables for the correlation of the fault tree input with the subrolutine.

Figure 3 is an example of a portion of a subroutine generated by TREBIL from the input illustrated on Figure 2. The logical array $X$ is used to represent the components, and the logical array $A$ is used to represent the logical gates of the fault tree.

In order to correlate the input names for lngiral. gates and oompo... nents, with the logical arrays $A$ and $X$ respectively, cross-reference tables are printed by TREBIL. For example, if TREBIL indexes a component with alphanumeric name COMPI as 5 , then the location $X(5)$ corresponds to that component, and if COMPI is in the failed state, $X(5)$ is given the value .TRUE.. This correspondence is indicated in a cross-reference table having the following format.

\begin{tabular}{|c|c|c|c|c|}
\hline Trep Tntex & Componont Name & LAMBD & & TAU \\
\hline 1 & COMPI & 1.00000 & D-06 & 0.0 \\
\hline 2 & COMPL5 & 1.5600 & $D-05$ & 1.0 \\
\hline - & . & . & & - \\
\hline$\cdot$ & . & - & & - \\
\hline
\end{tabular}


A similar cross-reference is printed for the gate names versus gate indexes .

In addition a table of components versus gates input by that component is printed to aid in correlation of the computer output with the original tree. This table is printed with the following format.

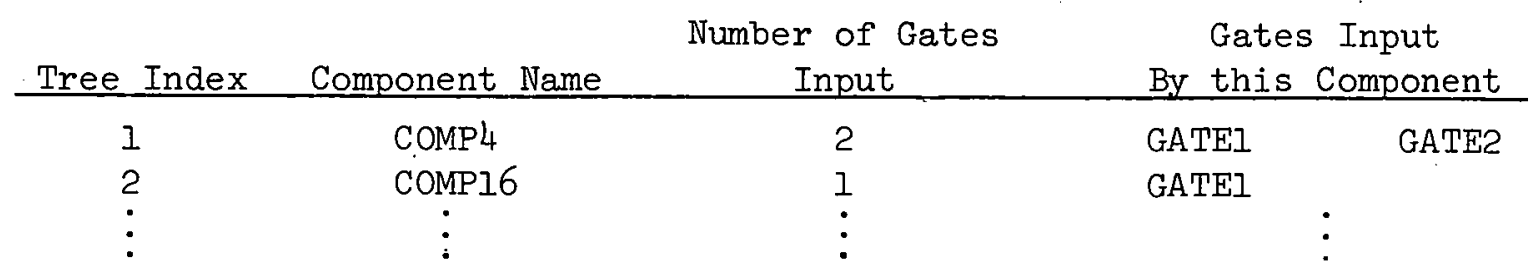

2. Output From MINSET

The output from MINSET consists of the obtained minimal cut sets or minimal path sets. This output may be printed only, or both printed and punched.

If COMBO has been used to obtain minimal cut sets, then each minimal cut set is printed and punched as it is found. An example of the output print format is as follows:

$$
\begin{aligned}
& \text { MINIMAL CUT SET NO. } 5 \\
& \text { COMPI }
\end{aligned}
$$

for each minimal cut set.

When FATE is used to obtain the minimal cut sets from the system the cut sets are printed twice. Each set is printed once as it is found by FATE. Also the complete collection of minimal cut sets is printed a second time ordered by the number of components in each set.

The output from FATE also includes the number of the Monte Carlo trial on which each cut set failed, a probability of failure for each cut set, and the index for each gate that is in the failed state as a result of the minimal cut set failure. 
The probability of failure which is printed for each cut set is useful in the determination of the relative importance of the cut sets. If IREN was input as 1 or 2 , then the printed probability represents the probability of failure up to time $t \cong 1.0 / \lambda_{\max }$. If IREN was input as 3 , then the printed probability represents the probability of failure up to time $t=T A A$. Caution must be used in attaching significance to this probability when TAA has been supplied by the user.

When minimal path sets are requested (IDEXI = 1) from MINSET, the output is analogous to that obtained for minimal cut sets except that probabilities are not computed.

If IDEX2 $=1$, then the obtained minimal cut or path sets will be punched in a format useable by the KITT program. The punched output includes the complete set of failure intensities and repair times for the tree's components as well as the cut or path sets. A card must be supplied with the number of cut or path sets punched in an Ilo format before the deck can be used in KITT (see KITT-I input description). 
THE USE OF THE KITT CODES

by

W. E. Vesely

IDAHO NUCLEAR CORPORATION

Idaho Falls, Idaho 


\section{INTRODUCTION}

KITT-1 is a computer code written as an application of Kinetic Tree Theory ${ }^{[a]}$. The code is written in FORTRAN IV, in double precision, for the IBM $360 / 75$ computer. KITT-1 can handle components which are nonrenairahtie or which have a constant repair time $\tau$. Any assortment of nonrepairable components and components having constant repair times can be considered. Any inhibit conditions having constant probabilities of occurrence can be handled. The failure intensity $(\lambda)$ of each component is assumed to be constant with respect to time (ie, exponential failure distributions are only considered $)^{[b]}$. As in Kinetic Tree Theory, the components are assumed independent. Further, all the components are assumed to be in their operating state at $t=0 . \mathrm{KITT}-1$ is a single phase component code; each component may have only one failure intensity $\lambda$ and one repair time $\tau$ for all operation time (or be nonrepairable for all time).

Besides the $\lambda$ and $\tau$ for each component, KITT-1 requires as input either the unique minimal cut sets of the fault tree or the unique minimal path sets of the fault tree. (A minimal cut set is a smallest set of components such that if all the components are simultaneously failed, the system is failed. A minimal path set is a smallest set of components such that if all the components are simultaneously functioning, the system is

\footnotetext{
[a] For the theoretical basis of Kinetic Tree Theory, the reader is referred to the report Analysis of Fault Trees by Kinetic Tree Theory [2].

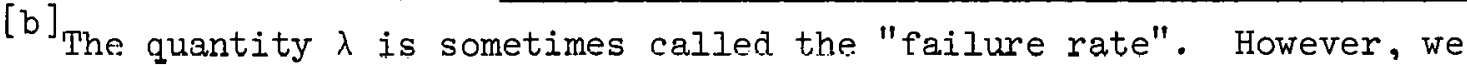
will reserve the term "failure rate" for another component quantity and will call $\lambda$ the "failure intensity".
} 
functioning.) The same reliability information is nhtained from the minimal cut sets or from the minimal path sets. Fxact, time-dependent reliability information is determined for each component of the fault tree and for each minimal cut set or path set. For the system, reliability results are obtained by upper bound approximations or by "bracketing". The upper bounds can be obtained when minimal cut sets are used to determine system reliability information. The upper bounds are not only upper. . bounds, but are also excellent approximations to the exact values. In the bracketing procedure, various upper and lower bounds are determined for the system results. The upper and lower bounds may be obtained as close to one another as desired; hence the true results may be enveloped as. tightly as desired. If the bracketing procedure is carried to its (finitie) completion, then the exact values for the system results are obtained.

\section{RELIABILITY INFORMATION OBTAINED BY KITT-T}

\section{Component Information}

For each component of the fault tree, KITT-1 obtains the following reliability thuracteristics:

$$
\begin{aligned}
& \mathrm{q}(\mathrm{t}) ; \text { the probability that the } \\
& \text { component is in its failed } \\
& \text { state at time } t
\end{aligned}
$$


The quantity $q(t)$ is termed the component failed probability; $q(t)$ is the probability that when the component is examined at time $t$, it is in its failed state at this point in time. The quantity $w(t)$ is termed the component failure rate. The integral of $w(t)$ over any time interval from $t_{1}$ to $t_{2}$ gives the expected number of times the component will fail during this interval. The integral of $w(t)$ from 0 to $t$, which is the third quantity in the above list, is thus the expected number of times the component will fail up to time $t$. The last quantity in the above list which is self-explanatory is a trivial computation, but saves the user the task of looking up a value for an exponential.

The quantities, or characteristics, $q(t)$ and $w(t)$ are termed differential characteristics since they are pointwise quantities, being obtained at a point in time. The quantities $\int_{0}^{t} w\left(t^{\prime}\right) d t^{\prime}$ and $I-\exp (-\lambda t)$ are termed integral characteristics.

The above four characteristics are obtained for each component at time points $t=t_{n}$, specified by the user.

\section{Minimal Cut Set Information}

If minimal cut sets are used to obtain system reliability information, then for each minimal cut set of the fault tree, the following reliability characteristics are obtained:

$$
\begin{aligned}
\check{Q}(t) \text {; the probability that the minimal } \\
\text { cut set is in its failed state at } \\
\text { time } t[a]
\end{aligned}
$$

\footnotetext{
[a] The superscript symbol " $\sim$ " is used to denote minimal cut set reliability quantities.
} 


$$
\begin{aligned}
& \check{\Lambda}(t) \text {; the probability that the min- } \\
& \text { imal cut set will suffer a } \\
& \text { failure per unit time at time } \\
& t \text { given it is in its function- } \\
& \text { ing state at time } t \text {. } \\
& \int_{0}^{t} W\left(t^{\prime}\right) d t^{\prime} \text {; the expected number of failures } \\
& \text { the minimal cut set will suffer } \\
& \text { during the time interval from } \\
& 0 \text { to } t \\
& 1-\exp \left[-\int_{0}^{t} \check{\Lambda}\left(t^{\prime}\right) d t^{\prime}\right] ; \text { the probability that the min- } \\
& \text { or more failures during the } \\
& \text { time interval from } 0 \text { to } t \text {. }
\end{aligned}
$$

A minimal cut set is said to be in its failed state if and only if all of its components are simultaneously in their failed state. A minimal cut set is thus in its functioning state if and only if one or more of its components is in its functioning state. A faiture of a minimal cut set is the passage of the minimal cut set from its functioning state to its failed state. (Consequently, a minimal cut set behaves like an "and gate" on a fault tree.)

The quantity $\dot{Q}(t)$ is called the minimal cut set faj]en probability. $\check{Q}(t)$ is the probability that all the minimal cut set components are simultianeously in their failed states at time $t$. Now the system is in its failed state if a minimal cut set is in its failed state. Therefore, $\check{Q}(t)$ for a particular minimal cut set is the probability that the system is in its failed state at time $t$ by means of the particular minimal cut set being in its failed state at time $t$. Examination of the $\check{Q}(t)$ for each of the minimal cut sets of the fault tree will determine the critical minimal cut sets, those with the largest $\check{Q}(t)$, by which the system is most likely to be in a failed state.

The quantity $\check{W}(t)$ is called the minimal cut set failure rate. The integral of $\breve{W}(t)$ over any time interval from $t_{1}$ to $t_{2}$ yields the expected 
number of times the minimal cut set will suffer a failure in this interval. The integral from 0 to $t$ is the expected number of times the minimal cut set will suffer a failure up to time $t$. The system will suffer a failure if a minimal cut set suffers a failure. The minimal cut sets with the largest $\check{W}(t)$ are therefore those which contribute most to system failure. The quantity $\check{\Lambda}(t)$ is termed the minimal cut set failure intensity. $\check{\Lambda}(t)$ is analogous to the component failure intensity $\lambda$ and is used in exactly the same way as $\lambda$ is used for a component. The quantity $\exp \left[-\int^{t} \tilde{\Lambda}\left(t^{\prime}\right) d t^{\prime}\right]$ is the probability that the minimal cut set suffers no failure to time $t$. The quantity $1-\exp \left[-\int_{0}^{t} \Lambda\left(t^{\prime}\right) d t^{\prime}\right]$ is therefore the probability that the minimal cut set suffers one or more failures up to time t.

The differential characteristics $\breve{Q}(t), \breve{W}(t)$, and $\check{\Lambda}(t)$ and the integral characteristics $\int_{0}^{t_{\tilde{W}}}\left(t^{\prime}\right) d t^{\prime}$ and $1-\exp \left[-\int_{0}^{t_{\tilde{L}}}\left(t^{\prime}\right) d t^{\prime}\right]$ are obtained for each minimal cut set at time points $t=t_{n}$ specified by the user.

\section{Minimal Path Set Information}

If minimal path sets are used to obtain system reliability information, then for each minimal path set the following reliability characteristics are obtained:

$$
\begin{aligned}
\hat{P}(t) ; & \text { the probability that the minimal } \\
& \text { path set is in its functioning } \\
& \text { state at time t[a] } \\
\hat{W}(t) ; & \text { the expected number of failures } \\
& \text { the minimal path set will suffer } \\
& \text { per unit time at time. } t
\end{aligned}
$$

\footnotetext{
[a] The superscript symbol." n " is used to denote minimal path set reliability quantities.
} 


$$
\begin{array}{ll}
\hat{\Lambda}(t) ; & \text { the probability that the minimal } \\
\text { path set will suffer a failure } & \text { per unit time at time } t \text { given it } \\
\text { is in its functioning state at } & \text { time } t
\end{array}
$$

A minimal path set is said to be in its functinning state if and only if all of its components are simultaneously in their functioning states. A minimal path set is thus in its failed state if and only if one or more of its components is in its failed state. A failure of a minimal path set is the passage of the minimal path set from its functioning state to its failed state. (A minimal path set therefore behaves like an "or gate" on a fault tree.)

The quantity $\hat{P}(t)$ is termed the minimal path sot functioning protuability. The system is in its functioning state if one of the minimal path sets is in its functioning state. Therefore, $\hat{\Gamma}(t)$ for a purt1cular minimal path set is the probability that the system is in its functioning state by means of the particular minimal path set being in its functioning state. Those minimal path sets with the largest $\hat{P}(t)$ are the critical minimal path sets by which the system is most likely to be in a functioning state.

The quantity $\hat{W}(t)$ is termed the minimal path set failure rate. $\hat{W}(t)$ is analogous to the minimal cut set failure rate and component failure pale and is used in the same way as the other failure rates.

The quantity $\hat{\Lambda}(t)$ is termed the minimal path set failure intensity. $\hat{\Lambda}(t)$ for a minimal path set is used in exactly the same way as $\hat{\Lambda}(t)$ is 
used for a minimal cut set and $\lambda(t)$ is used for a component. The quantity $\exp \left[-\int_{0}^{t} \hat{\Lambda}\left(t^{\prime}\right) d t^{\prime}\right]$ is the probability that the minimal path set suffers no failure to time $t$. Those minimal path sets with the largest value of $\exp \left[-\int^{t} \hat{\Lambda}\left(t^{\prime}\right) d t^{\prime}\right]$ are those critical minimal path sets by which the system 0 will most likely function to time $t$ without a failure.

The differential characteristics, $\hat{P}(t), \hat{W}(t)$, and $\hat{\Lambda}(t)$ and the integral characteristics $\int_{0}^{t} \hat{W}\left(t^{\prime}\right) d t^{\prime}$ and $\exp \left[-\int_{0}^{t} \hat{\Lambda}\left(t^{\prime}\right) d t^{\prime}\right]$ are obtained at time points $t=t_{n}$ specified by the user.

\section{System Reliability Information}

The fault tree can be described in an equivalent manner by either its minimal cut sets or by its minimal path sets; the same system reliability information, having exactly the same values, is obtained from either the minimal cut sets or the minimal path sets. The system reliability information, or system reliability characteristics, obtained from the minimal cut sets or path sets are:

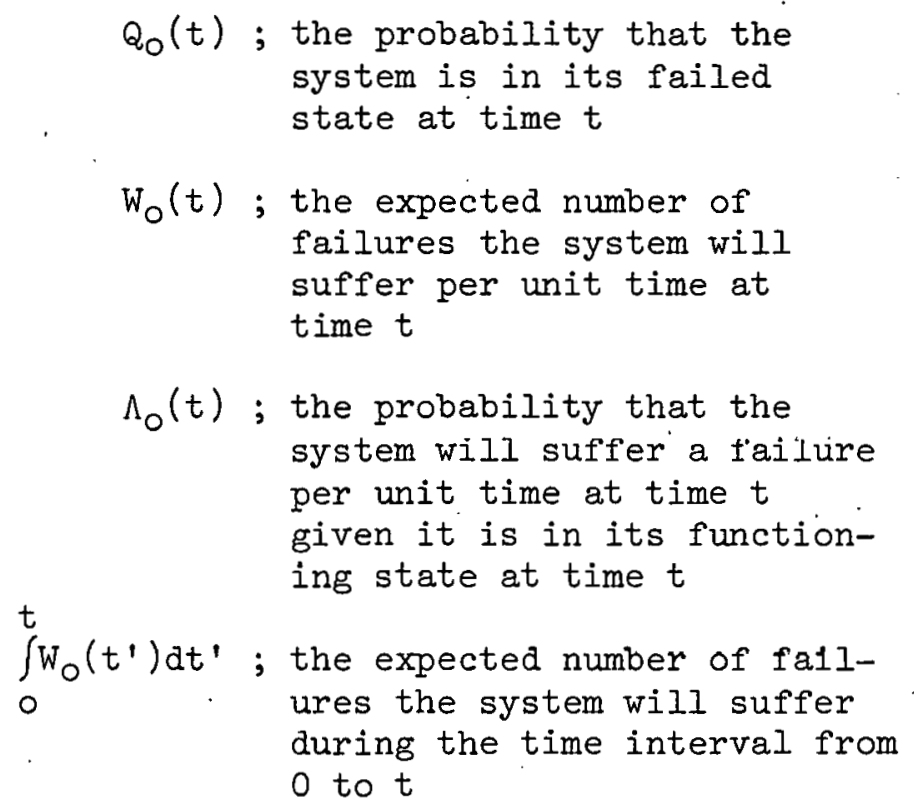




$$
\begin{aligned}
1-\exp \left[-\int_{0}^{t} \Lambda_{0}\left(t^{\prime}\right) d t^{\prime}\right] ; & \text { the probability that the } \\
& \text { system will suffer one or } \\
& \text { more failures in the time } \\
& \text { interval from } 0 \text { to } t .
\end{aligned}
$$

The quantity $Q_{0}(t)$ is called the system failed probability. If the system is examined at time $t$, then $Q_{0}(t)$ is the probability that the system is down, or in its failed state, at this particular time.

The quantity $W_{0}(t)$ is termed the system failure rate. The integral of $w_{0}(t)$ over any time interval from $t_{1}$ to $t_{2}$ gives the expected number of times the system will fail during this interval. The integral $\int_{0}^{t} w_{0}\left(t^{\prime}\right) d t^{\prime}$ is consequently the expected number of times the system will fail up to time $t$.

The quantity $\Lambda_{0}(t)$ is termed the system faidure intensity and is analogous to the other failure intensities discussed. The quantity $\exp \left[-\int_{0}^{t} \Lambda_{0}\left(t^{\prime}\right) d t^{\prime}\right]$ is the probability of no system failure to time $t$; and one minus this quantity, the last characteristic in the above list, is the probability that the system will suffer one or more failures to time $t$.

The differential system chararteristics $Q_{0}(t), W_{U}(t)$, and $\Lambda_{0}(t)$ and the integral system characteristics $\int_{0}^{t} w_{0}\left(t^{\prime}\right) d t^{\prime}$ and $I-\exp \left[-\int_{0}^{t} \Lambda_{0}\left(t^{\prime}\right) d t^{\prime}\right]$ are obtained at the same time points as the component and set characteristics, $t=t_{n}$, which are specified by the user. The systom charaotcriotico are obtained either by upper bound approximations or by bracketing. If the bracketing is carried to its completion then the exact values for these system characteristics are obtained.

\section{INPUT DESCRIPTION}

This section describes the data which must be input to KITT-1. A detailed description of this input, along with recommendations, is given. 
All the integer input data (fixed point data) are read under an Ilo format and therefore must be right justified. All the decimal input data (floating point data) are read under an Fl0.0 format. This format will take any decimal number, such as 15.0 , which can be situated anywhere within the 10 columns, but decimal points must be punched. It will also take the $E$ or $\mathrm{D}$ representation, such as $1.5 \mathrm{D}+01$, but the decimal point must be punched and the number must be right justified.

\section{Input Group 1}

Number of Cards: 1

Format: $10 \mathrm{~A} 8$

Input Data: Title Card

This card is used for description of the problem to be run. Any alphanumeric characters may be used in the 80 columns of the card.

\section{Input Group 2}

Number of Cards: 1

Format: II0

Input Data: NPROB (NPROB $\geq 1)$

The parameter NPROB is the number of "parameter runs" to be performed for the same fault tree (ie, for the same minimal cut sets or minimal path sets). A parameter run is the fault tree analyzed with the components having a particular set of values of $\lambda$ and $\tau$. For NPROB $=1$, the fault tree is analyzed with one set of $\lambda^{\prime} s$ and $\tau^{\prime}$ 's used for the components. For NPROB $=n$, with $n>1$, the same fault tree is analyzed with $n$ different sets 
of values of $\lambda^{\prime}$ 's and $\tau$ 's for the components. For NPROB greater than 1 , one can therefore analyze the differences in system reliability due to changes of various component $\lambda^{\prime} s$ and $\tau$ 's.

\section{Input Group 3}

Number of Cards: 1

Format: II0

Input Data: $\operatorname{NCOMP}(1 \leq \mathbb{N C O M P} \leq 400)$

NCOMP is the total number of unique components plus unique inhibit conaltions. 'l'his total number of' components plus inhibit conditions must include the number of unique components plus inhibit conditions which are in one or more of the minimal cut sets or minimal path sets. If NCOMP is greater than the number in the minimal cut sets or path sets, (component) reliability information is obtained for those components not in any minimal cut set or path set. These components not in any minimal sut set or path set are ignored in the system reliability computations.

\section{Input Group 4}

Number of Cards: Varies

Format: 8 F10.0

Input Data: XLMDA(I); $I=1, \operatorname{NCOMP}$

$\mathrm{XLMDA}(I)$ is the failure intensity $\lambda$, (per hour) for the component of index I. The failure intensities $\lambda$ are read in, 8 to a card, in order of increasing component index. The $\lambda$ 's read in here are for the first parameter run (see Input Group 2). For identification, each unique component or unique inhibit condition is assigned a unique index I, where I ranges 
from 1 to NCOMP. [NCOMP is the total number of components and inhibit conditions (see Input Group 3).] The assignment of indices is arbitrary; if the PREP code is used to determine the minimal cut sets or minimal path sets, the component indices are automatically assigned by it. A nonpositive $\lambda_{j}\left(\lambda_{j} \leq 0\right)$ denotes the "component" of index $j$ is an inhibit condition.

\section{Input Group 5}

Numher of Carris: Varies

Format: 8 F].0.0

Input Data: $\operatorname{TAU}(\mathrm{I}): \mathrm{I}=1$, NCOMP

$\mathrm{TAU}(I)$ is the constant repair time $\tau$ (hours) for the component of index I. The $\tau$ 's read in here are for the first parameter run. The repair times $\tau$ are read in, 8 to a card, in order of increasing component index. A nonpositive $\tau(\tau \leq 0)$ denotes the component is nonrepairable. In the preceding input group (Input Group 4), a nonpositive $\lambda, \lambda_{j} \leq 0$, denoted that component $j$ is an inhibit condition. If this is the case, then $\tau_{j}$ is interpreted as the probability of occurrence for this respective inhibit wondition and must be read in as such. A quick reference is given below in the use of $\lambda$ and $\tau$ to denote nonrepairable components, inhibit conditions, etc.

Variables Input

$$
\lambda>0, \tau>0
$$$$
\lambda>0, \tau \leq 0
$$

\section{Meaning}

A repairable component. The value $\lambda$ is then the value of the failure intensity (per hour) and $\tau$ is the value of the repair timc (hours) for this component.

A nonrepairable component. The value $\lambda$ is then the value of the failure intensity (per hour) for this component. 
Variables Input

$\lambda \leq 0, \tau \geq 0$
Meaning

An inhibit condition. The value $\tau$ is then the value of the occurrence probability for this inhibit condition.

\section{Input Group 6}

Number of Cards: 1

Format: $\quad$ I10

Input Data: ISTOP

ISTOP is the bracket flag and is read in as either 1 or 2 . If ISTOP $=2$, system reliability characteristics $Q_{0}(t), W_{0}(t)$, etc, are obtained by means of the bracketing procedure. If ISTOP $=1$, the bracketing procedure is not used. (The bracketing procedure is explained in Input Group 9.)

Regardless of the value of ISTOP, reliability characteristics are always determined for the components and minimal cut sets or path sets. If reliability characteristics are desired only for the components and minimal cut sets or path sets, but not for the system, set ISTOP $=1$ [a]. II system characteristics are desired and minimal path sets are used to obtain these characteristics then ISTOP must be set equal to 2 . If system characteristics are wanted and minimal cut sets are input, then ISTOP can be set equal to 1 or 2 . For ISTOP $=2$, upper and lower bounds are obtained for the system characteristics and if this bounding is carried to its completion the exact values for these characteristics are obtained. This is explained in more detail in Input Groups. 9 and 10. For ISTOP $=1$, only upper bounds are obtained for the system characteristics. These upper bounds are explained in more detail below.

[a] ISTOP should also be set equal to 1 if input data are merely to be checked for any errors. 
As stated above, if minimal cut sets are used to obtain system reliability characteristics, then ISTOP can be set equal to I to obtain upper bounds for the system characteristics. These upper bounds are obtained in a very small amount of computer time (on the order of fractions of a minute). Furthermore, the upper bounds are also excellent approximations to the exact values of the system characteristics. For a system whose components are repairable, the fractional errors made are on the order of $0.1 \%$ for differential results $\left[W_{O}(t), Q_{O}(t), \Lambda_{O}(t)\right]$. For a system whose components are all nonrepairable, the fractional errors made for the differential characteristics are on the order of $10 \%$. For ISTOP $=1$, the system failure rate $W_{0}(t)$, failure intensity $\Lambda_{0}(t)$, and failed probability $Q_{0}(t)$ are bounded (and approximated) as: ${ }^{[a]}$

$$
\begin{aligned}
& Q_{0}(t) \leq 1-\prod_{i=1}^{N_{c}}\left[1-\check{Q}_{i}(t)\right] \\
& W_{O}(t) \leq \sum_{i=1}^{N_{c}} \check{W}_{i}(t) \\
& \sum_{i=1}^{N_{c}} \check{W}_{i}(t) \\
& \prod_{i=1}^{N_{c}}\left[1-\check{Q}_{i}(t)\right]
\end{aligned}
$$

where

$$
\begin{aligned}
\mathbb{N}_{c} & =\text { total number of minimal cut sets } \\
\check{W}_{i}(t) & =\text { the } i^{\text {th }} \text { minimal cut set failure rate } \\
\check{Q}_{i}(t) & =\text { the } i^{\text {th }} \text { minimal cut set failed probability. }
\end{aligned}
$$

\footnotetext{
[a] For a derivation of these bounds, and a proof that they are always upper bounds, see the report Analysis of Hault Trees by Kinetic Tree Theory [2].
} 
Using these upper bounds for $\Lambda_{0}(t)$ and $W_{0}(t)$, upper bounds for the integral system characteristics are also output;

$$
\int_{0}^{t} w_{0}\left(t^{\prime}\right) d t^{\prime} \leq \int_{0}^{t}\left\{\sum_{i=1}^{N_{C}} \check{w}_{i}\left(t^{\prime}\right)\right\} d t^{\prime}
$$

and

$$
I-\exp \left[-\int_{0}^{t} \Lambda_{\odot}\left(t^{\prime}\right) d t^{\prime}\right] \leq 1-\exp \left[-\int_{0}^{t}\left(\frac{\sum_{i=1}^{N_{c}} \underline{W}_{i}\left(t^{\prime}\right)}{\prod_{i=1}^{N_{c}}\left(I-\check{Q}_{i}\left(t^{\prime}\right)\right)}\right) d t^{\prime}\right]
$$

If results from bracketing are obtained, by setting ISTOP $=2$, and - minimal cut sets are used, the upper bounds given by Equations (I) through (3) and by Equations (7) and (8) are still output as additional information since the additional computer time needed to obtain these upper bounds is negligible.

(A more detailed treatment of the accuracy of these upper bounds is given lin subsection '2, on page 31 , a subsection of the recommendations given there.)

\section{Input Group 7}

Number of Cards: 1

Format: $2 I 10, F 10.0$

Input Data: NTPT; NOUT, DELTA. $2 \leq \mathrm{NTPT} \leq 50$

NOUT $\geq 1$

This input consists of three numbers punched on a card. NTPT is the total number of time points at which reliability characteristics are obtained, NOUT is the printout multiple, and DELTA is the spacing between the time points. If DELTA is given a positive value $\Delta t$ (in hours) then 
characteristics are obtained at times $0, \Delta t, 2 \Delta t, \ldots,(\mathrm{NTPT}-1) \Delta t$. If DELTA is given a negative or zero value, then the time points are explicịtly input (Input Group 8), and reliability characteristics will be obtained for these time points read in.

The reliability characteristics for components, minimal cut or path sets, and for the system are all obtained at the same time points. NTPT is the total number of time points at which these characteristics are obtained. This number of time points is arbitrary, but it must not exceed 50. In most cases, the number of time points used does not significantly affect the computer time. However, for assurance, it is a good practice to always use the minimum number of time points for the accuracy desired. NOUT is the printout multiple. If NOUT is set equal to 1 , component and minimal cut or path set characteristics are printed out for every time point; if NOUT is set equal to 2 , they are printed out for every second time point, and so forth. System reliability characteristics are always printed out for every time point. NOUT need not be evenly divisible into NTPT.

If DELTA is read in as a positive value $\Delta t$, it is taken to be the spacing, in hours, between the time points at which characteristics are obtained. The time points are therefore evenly spaced. If DELTA is positive, the first time point is always 0.0 and therefore the NTPT time point, the last time point, is (NTPT - 1) $\Delta$ t.

If DELTA is read in as 0.0 , or any negative number, then the NTPT time points, arbitrarily spaced, are explicitly input in the next input group. The flexibility of explicitly reading in the time points at which characteristics are obtained allows the user to place the time points so that maximum detail and accuracy are obtained for a problem. 
The time points used do not in any way affect the accuracy of the differential reliability characteristics (failed probability, failure rate, and failure intensity), which are obtained for the components, sets, and the system. The integral characteristics, however, are obtained using a trapezoidal integration over the pertinent differential characteristics, using only those differential characteristics at the time points chosen. If accurate integral characteristics are desired, then preferably DELTA should be set equal to a negative number (or zero) and the time points should explicitly be read in. The placing of these time points is alscussed in the next input group. If DELTA is set equal to a positive number, then to obtain accurate integral characteristics, NTPT should be set equal to 50 and DELTA should be determined from the formula

$$
\text { DELTA }=\frac{t_{\max }}{49}
$$

where $t_{\max }$ is the maximum time of interest for which characteristics are desired.

\section{Input Group 8}

Number of Cards: Varies

Format: 8 F10.0

Input Uata: TOT $(\mathrm{J}) ; \mathrm{J}=1$, NTPT

This Input Group is skipped if DELTA is positive (Tnput Group 7).

$\operatorname{TO}^{\prime}(J)$ are the time points at which the component, set, and system characteristics are obtained, if DELTA is negative or zero. Eight time points are read in on a card, and are continued on succeeding cards until the NTPT time points are read in. The time points may be arbitrarity 
spaced and may begin at any nonnegative time $[$ TOT(I) $\geq 0.0]$. However, the time points must be in increasing order $[\operatorname{TOT}(1)<\operatorname{TOT}(2)<\operatorname{TOT}(3)<\ldots<$ TOT(NTPT)]. Also, the integral characteristics are always evaluated using TOT(I) as the beginning time.

The integral characteristics are always obtained from integrations, beginning with the first time point which is input, TOT(1). [For all the integral characteristics for components, sets, and the system on pages 2 , 3,5 , and 7 , respectively, replace the bottom time on the integrals from $t=0$ to $t=t_{0}$, where $t_{0}=\operatorname{TOT}(1)$. The definitions would then read "in the time period from $t_{0}$ to $\left.t^{\prime \prime}.\right]$ Therefore, unless the user has a definite reason for not doing so, TOT(I) should be read in as 0.0 .

As stated in the previous input group, the accuracy of the differential characteristics is not affected by the placing of the time points, but the accuracy of the integral characteristics is. Therefore, for accurate integral characteristics the time points should be chosen so that the points are closely spaced in areas where the differential characteristics are rapidly changing in value. This generally involves spacing the points closely near TOT(I), the origin, and further apart for greater times.

If a component has a constant repair time $\tau$, then its differential characteristics will go asymptotic, or assume constant values, after several multiples of $\tau($ from $t=0.0)$. Therefore, for a system with repairable components, it is best to have the first several time points spaced $\tau_{\mathrm{a}}$ apart, where $\tau_{\mathrm{a}}$ is a characteristic component repair time. After this transient, period, the time points can then be spaced as far apart as desired, which will not affect the accuracy of the integral characteristics since the differential characteristics will have assumed their constant, "steady state" values. If the components are nonrepairable, then the 
differential characteristics change smoothly in value and an even spacing of the time points is generally best.

\section{Input Group 9}

Number of Cards: 1

Format: $2 I 10$

Input Data: NBMAX, IFAG2 ( $1 \leq$ NBMAX $\leq 400)$

This Input Group is skipped if

ISTOP $=1$ (Input Group 6).

NBMAX is the number of outer brackets to obtain. TFAF? is tho oyotcm failure rate correction flag and must have a value of 1 or 2 . If IFAG2 = 2 , the system failure rate correction term $\left[\tilde{\mathrm{w}}_{0}^{(2)}(t)\right.$ for minimal cut sets or $\hat{\mathrm{W}}_{0}^{(2)}(t)$ for minimal path sets] is bracketed. If IFAG2 $=1$, this correction term is not computed. If minimal path sets are input and system reliability information is desired (TSTOP $\equiv 2$ ), then NBMAX and IFAG2 must have only one set of values as given bolow. If minimal cul sels are input and ISTOP $=2$, the choice of values for NBMAX and IFAG2 is somewhat arbitrary, and their use in the code is described below. The user who merely wishes efficient, predetermined values for these two variables is referred to the recommendations given on page 30 or to the recommendation summary on page 41 .

\section{A. Minimal Cut Sets Input}

If minimal cut sets are uegd to detcrmine syslem charactéristics then the system failed probability $Q_{0}(t)$ is equal to the probability of one or more of the minimal cut sets being failed at time $t^{[2]} \cdot Q_{0}(t)$ can thus be expressed as 


$$
\begin{gathered}
Q_{0}(t)=Q_{1}(t)-Q_{2}(t)+Q_{3}(t) \\
-\ldots(-1)^{\mathbb{N}_{C}-1} Q_{\mathbb{N}_{C}}(t)
\end{gathered}
$$

where

$$
\begin{array}{r}
\mathrm{N}_{c}=\text { the total number of minimal } \\
\text { cut sets for the fault tree }
\end{array}
$$

and

$$
\begin{aligned}
Q_{n}(t)= & \text { the contribution to } Q_{o}(t) \text { from } \\
& n \text { minimal cut sets being simul- } \\
& \text { taneously failed at } t(n=1, \\
& \left.\ldots, N_{c}\right) .
\end{aligned}
$$

$Q_{n}(t)$ is thus given by the expression

$$
Q_{n}(t)=\sum_{i_{1} \cdots i_{n}} Q\left(i_{1} \ldots i_{n} ; t\right)
$$

where $Q\left(i_{1} \ldots i_{n} ; t\right)$ is the probability of the $n$ minimal cut sets $i_{1}, \ldots, i_{n}$ being simultaneously failed at $t$ and the summation is over all combinations of the $\mathrm{N}_{\mathrm{C}}$ minimal cut sets taken $\mathrm{n}$ at a time.

The system failure rate $W_{0}(t)$ is given by the expression

$$
w_{0}(t)=\check{W}_{0}^{(1)}(t)-\check{w}_{0}^{(2)}(t) .
$$

$\tilde{W}_{0}^{(1)}(t)$ is the major contribution to $W_{0}(t)$ while $\tilde{W}_{0}^{(2)}(t)$ is a correction term. $\left[\tilde{W}_{0}^{(2)}(t)\right.$ is generally less than $1 \%$ of $\left.\tilde{W}_{0}^{(1)}(t).\right] \tilde{W}_{0}^{(1)}(t)$ is equal to the probability of one or more minimal cut sets failing at time $t$, and can be expressed as

$$
\begin{aligned}
\tilde{W}_{0}^{(1)}(t) & =W_{1}^{(1)}(t)-W_{2}^{(1)}(t)+W_{3}^{(1)}(t) \\
& -\ldots+(-1)^{N_{C}-1} W_{N}^{(1)}(t)
\end{aligned}
$$

where

$$
\begin{aligned}
\mathrm{W}_{\mathrm{n}}^{(1)}(\mathrm{t})= & \text { the failure rate for exactly } \\
& \text { n minimo, cut sets simulta- } \\
& \text { neously failing at } t(\mathrm{n}=1, \\
& \left.\ldots, \mathrm{N}_{\mathrm{c}}\right) .
\end{aligned}
$$


Analogous to $Q_{n}(t), W_{n}^{(1)}(t)$ is given by the expression

$$
w_{n}^{(1)}(t)=\sum_{i_{1} \ldots i_{n}} w\left(i_{1}, \ldots i_{n} ; t\right)
$$

where $W\left(i_{1}, \ldots i_{n} ; t\right)$ is the probability of the $n$ minimal cut sets $i_{1}, \ldots, i_{n}$ simultaneously failing at $t$. The summation again is over all combinations of n minimal cut sets.

NBMAX of Input Group 9 involves the computation, or "bracketing", of $Q_{0}(t)$ and $\check{W}_{0}^{(l)}(t)$. For $\operatorname{NBMAX}=K$, where $K$ is less than or equal to the total number of minimal cut sets $\mathbb{N}_{C}$, the first $K$ terms on the right-hand sides of Equations (10) and (15) are only considered to obtain K "brackets", or bounds, for $Q_{0}(t)$ and $W_{0}^{(1)}(t)$. For $Q_{0}(t)$, considering the first term on the right hand side of Equation (10) gives a first upper bound for Qo $(t)$,

$$
Q_{0}(t) \leq Q_{1}(t)
$$

Considering the first two terms gives a first lower hound for $Q_{U}(t)$,

$$
Q_{0}(t) \geq Q_{j}(t)-Q_{2}(t)
$$

Considering the first three terms gives a second upper bound for $Q_{O}(t)$,

$$
Q_{0}(t) \leq Q_{1}(t)-Q_{2}(t)+Q_{3}(t)
$$

Considering the first four terms gives a second lower bound for $Q_{0}(t)$, and so forth. This bracketing can continue until all $\mathrm{N}_{c}$ terms are considered. which then. not only is an upper or lower bound for $Q_{o}(t)$ but also is its exact value ${ }^{[a]}$. For NBMAX $=K$ the bracketing is continued only until $K$ terms are considered for $Q_{O}(t)$, yielding $K$ upper and lower bounds for

\footnotetext{
[a] For a general discussion and verification of this bracketing procedure, see the report Analysis of Fault Trees by Kinetic Tree Theory.
} 
$Q_{O}(t)$; for $K<\mathbb{N}_{C}$ the bracketing is thus truncated and is not carried to its completion (until $\mathrm{N}_{\mathrm{C}}$ terms are considered yielding the exact answer).

When NBMAX $=K$, in addition to the $K$ bounds obtained for $Q_{0}(t)$, from Equation (15) K bounds are obtained for $\check{\mathrm{W}}_{\mathrm{O}}^{(1)}(t)$;

$$
\begin{aligned}
& \check{W}_{0}^{(1)}(t) \leq w_{1}^{(1)}(t) \\
& \check{W}_{0}^{(1)}(t) \geq W_{1}^{(1)}(t)-w_{2}^{(1)}(t) \\
& \check{w}_{0}^{(1)}(t) \leq w_{1}^{(1)}(t)-w_{2}^{(1)}(t)+w_{3}^{(1)}(t) . \\
& \vdots
\end{aligned}
$$

As with $Q_{O}(t)$, the bounding, or bracketing, continues until $K$ terms are considered on the right-hand side of Equation (15).

From the $K$ terms considered for $Q_{0}(t)$ and $\tilde{W}_{0}^{(1)}(t)$, a number of upper and lower bounds are obtained for $Q_{O}(t)$ and $\check{W}_{O}^{(l)}(t)$. More precisely, for $K$ even, $\frac{K}{2}$ upper bounds and $\frac{K}{2}$ lower bounds are obtained for $Q_{0}(t)$ and $\check{\mathrm{W}}_{\mathrm{O}}^{(l)}(\mathrm{t})$. For $\mathrm{K}$ odd, $\frac{\mathrm{K}+\mathrm{I}}{2}$ upper bounds and $\frac{\mathrm{K}-\mathrm{I}}{2}$ lower bounds are obtained. From these upper and lower bounds, the code then chooses the best (smallest) upper bound and the best (largest) lower bound for $Q_{0}(t)$ and $\tilde{W}_{0}^{(1)}(t)$;

$$
\begin{gathered}
Q_{O}(t)_{\min } \leq Q_{O}(t) \leq Q_{O}(t)_{\max } \\
\check{W}_{O}^{(l)}(t)_{\min } \leq \check{W}_{O}^{(l)}(t) \leq \check{W}_{O}^{(l)}(t)_{\max }
\end{gathered}
$$

where $Q_{0}(t)_{\min }, Q_{0}(t)_{\max }, \check{W}_{0}^{(1)}(t)_{\min }, \check{W}_{0}^{(1)}(t)_{\max }$ are the best bounds chosen by the program from the $K$ bounds which were obtained by setting NBMAX $=K$. For $\mathrm{K}=\mathrm{N}_{\mathrm{C}}$, where $\mathrm{N}_{\mathrm{c}}$ is the total number of minimal cut sets, then one of the best bounds obtained is the exact value for $Q_{0}(t)$ and $\check{W}_{0}^{(}(1)(t)$. Depending upon whether $\mathbb{N}_{C}$ is even or odd, 


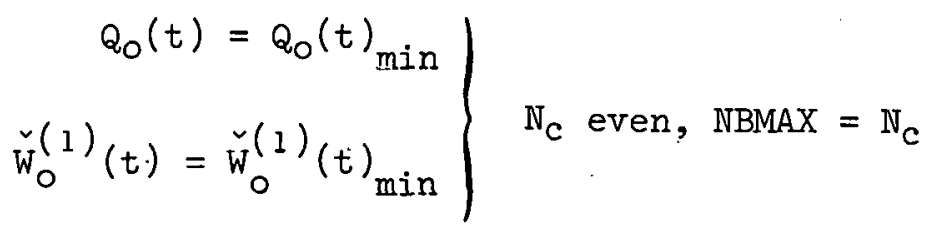

or

$$
\left.\begin{array}{l}
Q_{0}(t)=Q_{0}(t)_{\max } \\
(t)=\tilde{W}_{0}^{(1)}(t)_{\max }
\end{array}\right\} \quad N_{C} \text { odd, NBMAX }=\mathbb{N}_{C}
$$

As NBMAX is increased in value from 1 to $N_{C}$, the best upper and lower bounds chosen by the program will approach one another, giving tighter brackets for $Q_{O}(t)$ and $W_{O}^{(1 .)}(t)$. However, as NBMAX increases, computer times increase--sometimes dramatically. For $\mathbb{N}_{c}$ (the number of minimal cut sets) less than 10 , the exact values for $\check{W}_{0}^{(1)}(t)$ and $Q_{0}(t)$, obtained by setting NBMAX $=N_{c}$, can be determined in about one minute computer time. For general $N_{C}$, setting NBMAX = 1 will give only an upper bound for $Q_{0}(t)$ and $\tilde{W}_{0}^{(l)}(t)$ (the lower bounds being zero). These upper bounds will be larger (and hence poorer) than those obtained by setting ISTOP $=1$ (Input Group 6). Hence, if TSTOP is set equal to 2, NBMAX should be set at least equal to 2. A value of 2 is the smallest value NBMAX can have to obtain bulh un upper and (nonzero) lower bound for $Q_{0}(t)$ and $\ddot{W}_{0}^{\prime}(1)(t)$. Even for NBMAX set equal only to 2, this upper and lower bound will generally differ by at most $1 \%$, giving tight brackets for $Q_{0}(t)$ and $\tilde{W}_{0}^{(1)}(t)$.

For minimal cut sets input, NBMAX is thus a "convergence parameter" for $Q_{0}(t)$ and $\tilde{W}_{0}^{(1)}(t)$. The other variable of Input Group 9, IFAG2 is related to the corvergence, or bracketing, of the correction term $\tilde{W}_{0}^{(2)}(t)$ in Equation (14). If IFAG2 is read in as 1 , the correction term $\tilde{W}_{0}^{(2)}(t)$ is not computed. Therefore, setting IFAG2 = 1 will only gi.ve an upper bound for $w_{0}(t)$, no matter how tightly $\tilde{w}_{0}^{(1)}(t)$ is bracketed. If IFAG2 is read in as 2 , the correction term $\tilde{W}_{0}^{(2)}(t)$ is bracketed, allowing both 
upper and lower bounds to be obtained for the system failure rate $w_{0}(t)$ [or allowing the exact value of $w_{0}(t)$ to be obtained]. For IFAG2 $=2$, the variables of the next input group, Input Group 10, determine how tightly $\mathrm{w}_{0}^{(2)}(t)$ is bracketed.

\section{B. Minimal Path Sets Input}

If minimal path sets are used to obtain system reliability characteristics, then NBMAX should be set equal to the total number of minimal path sets $\mathbb{N}_{\mathrm{p}}$ and IFAG2 should be set equal to 2. For minimal path sets the bounds determined by the program for NBMAX $<\mathbb{N}_{p}$ are useless (greater than 1 and less than 0 ), and NBMAX must be set equal to $N_{p}$ to obtain not only the exact results but any reasonable results. For minimal path sets and NBMAX $=N_{p}$, the system failed probability $Q_{O}(t)$ and failure rate $W_{o}(t)$ are given exactly by one of the bounds determined by the code, depending upon whether $\mathrm{N}_{\mathrm{p}}$ is odd or even;

$$
\begin{aligned}
Q_{o}(t) & =Q_{o}(t)_{\min } ; N_{p} \text { even, NBMAX }=N_{p} \\
& =Q_{o}(t)_{\max } ; N_{p} \text { odd, NBMAX }=N_{p}
\end{aligned}
$$

and

with

$$
W_{0}(t)=\hat{W}_{0}^{(1)}(t)-\hat{W}_{0}^{(2)}(t)
$$

$$
\begin{aligned}
\hat{W}_{0}^{(1)}(t) & =\hat{W}_{o}^{(1)}(t)_{\min } ; N_{p} \text { even, NBMAX }=N_{p} \\
& =\hat{W}_{0}^{(1)}(t)_{\max } ; N_{p} \text { odd, NBMAX }=N_{p}
\end{aligned}
$$

where $Q_{0}(t)_{\max }, Q_{0}(t)_{\min }, \hat{W}_{0}^{(1)}(t)_{\min }, \hat{W}_{0}^{(1)}(t)_{\max }$ are the best bounds determined by the program. $\hat{\mathrm{W}}_{\mathrm{O}}^{(2)}(t)$ for $\operatorname{NBMAX}=\mathrm{N}_{\mathrm{p}}$ and IFAG2 $=2$ will also be determined exactly as discussed in the next input group. Because NBMAX must be set equal to $\mathrm{N}_{\mathrm{p}}$, minimal path sets should be used to obtain system 
reliability information only when there are a small number of such sets for a given fault tree $\left(N_{p} \leq 10\right)$.

\section{Input Group 10}

Number of Cards: Varies

Format: 8110

Input Data: $\mathrm{NB2}(\mathrm{N}) ; \mathrm{N}=1$, NBMAX $(I \leq \mathrm{NB} 2(\mathrm{~N}) \leq 100)$

This Input Group is skipped if IFAG2

$\equiv ' 1$ (Input Group 9), or if'I3TOP = 1 (Input Group 6).

$\mathrm{NB} 2(\mathbb{N})$ is the number of inner brackets to obtain for each outer bracket N. If minimal path sets are input and system reliability information is wanted, then $\mathrm{NB2}(\mathbb{N})$ must have only one set of values and these are given below. For minimal cut sets input, the way $\mathbb{N B 2}(\mathbb{N})$ is used in the code is described below, which is a continuation of the discuerione of Input Group 9. Again, the user who merely wishes effisient, reoommondca values for. NB2(N.) is referred to the recommendations on page 30 or to the recommendation summary of page 41 .

\section{A. Minimal Cut Sets Input}

If minimal cut sets are used to obtain system reliability information, then by setting IFAG2 $=2$ the "correction term". $w_{0}^{(}(2)(t)$ is cumputed [Equation (14) in Input Group 9]. The correction term $\check{w}_{0}^{(2)}(t)$ may be expressed as

$$
\check{w}_{0}^{(2)}(t)=w_{1}(t)-w_{2}(t)+w_{3}(t)-\ldots+(-1)^{N_{c}-1} w_{N_{c}}(t)
$$


where $\mathrm{N}_{\mathrm{C}}$ is the number of minimal cut sets and

$$
\begin{aligned}
W_{n}(t)= & \text { the failure rate for exactly } n \\
& \text { minimal cut sets simultaneously } \\
& \text { failing at time } t \text { with one or } \\
& \text { morc of the other minimal cut } \\
& \text { sets being in their failed states } \\
& \text { at time } t\left(n=1, \ldots, N_{c}\right) .
\end{aligned}
$$

$W_{n}(t)$ is analogous to the failure rate $W_{n}^{(1)}(t)$ of Equation (16); however $W_{n}(t)$ involves not only the $n$ minimal cut sets simultaneously failing, but also includes the probability of one or more of the other $N_{C}-n$ minimal cut sets being in their failed states at time $t$. Analogous to Equation (17), $W_{n}(t)$ can be expressed as

$$
w_{n}(t)=\sum_{i_{1}} w\left(i_{1}, \ldots i_{n} ; t\right) P_{u}\left(i_{1}, \ldots i_{n} ; t\right)
$$

where $W\left(i_{1}, \ldots i_{n} ; t\right)$ is the same as defined for Equation (17) and $P_{u}\left(i_{1}, \ldots i_{n} ; t\right)$ is the probability of one or more of the other minimal cut sets being failed at $t$ given the failure of the $i_{1}, \ldots i_{n}$ minimal cut sets. The summation is again over all combinations of $\mathrm{N}_{c}$ minimal cut sets taken $\mathrm{n}$ at a time.

$$
\begin{aligned}
& \text { Since } P_{u}\left(i_{1}, \ldots i_{n} ; t\right) \text { involves a union, } W_{n}(t) \text { can be written as } \\
& \qquad \begin{aligned}
W_{n}(t) & =W_{n}^{1}(t)-W_{n}^{2}(t)+W_{n}^{3}(t) \\
& \ldots+(-1)^{N_{C}-1} w_{n}^{N_{c}}(t)
\end{aligned}
\end{aligned}
$$

where

$$
\begin{aligned}
\mathrm{w}_{\mathrm{n}}^{\mathrm{m}}(t)= & \text { the failure rate for exactly } \\
& \mathrm{n} \text { minimal cut sets simulta- } \\
& \text { neously failing at time } t \text { with } \\
& \text { exactly } \mathrm{m} \text { other minimal cut } \\
& \text { sets being in their failed } \\
& \text { states at time } t\left(\mathrm{~m}=1, \ldots \mathrm{N}_{\mathrm{c}}\right)
\end{aligned}
$$

From Equation (35), 


$$
w_{n}^{m}(t)=\sum_{i_{1}}, \ldots i_{n} w\left(i_{1}, \ldots i_{n} ; t\right) p_{m}\left(i_{1}, \ldots i_{n} ; l\right)
$$

where $P_{m}\left(i_{1}, \ldots i_{n} ; t\right)$ is the probability of exactly m minimal cut sets being failed at time $t$ given the failure of the $i_{1} \ldots i_{n}$ minimal cut sets. In order to fail at time $t$, a minimal cut set must be in its functioning state at time $t$, and hence

$$
w_{n}^{m}(t)=0 ; m>N_{c}-n
$$

Equations (33), (34), (36), and (37) will be the equations of interest in the proceeding discussions.

For IFAGL $=2$ and $\mathrm{NB2}(\mathrm{N})=\mathrm{J}$, J terms are only considered on the right-hand side of Equation (36) where the index $\mathbb{N}$ is the same as the index $n$ in the above equations $(N=n)$. From these $J$ terms, $J$ brackets, or bounds, are obtained for $W_{n}(t)$;

$$
\begin{aligned}
W_{n}(t) & \leq W_{n}^{1}(t) \\
& \geq W_{n}^{1}(t)-W_{n}^{2}(t) \\
& \leq W_{11}^{1}(t)-W_{n}^{2}(t)+W_{n}^{3}(t) \\
& \text { etc. }
\end{aligned}
$$

This, proceeds until $\mathrm{J}$ terms are óbtained on the right-hand side of the abuve equations. For $J$ even, there are thus $\frac{J}{2}$ upper bounds and $\frac{J}{2}$ lower bounds obtained for $W_{n}(t)$. For $J$ odd, there are $\frac{J+1}{2}$ upper bounds and $\frac{\mathrm{J}-\mathrm{l}}{2}$ lower bounds obtained. From these $\mathrm{J}$ upper and lower bounds, which were obtained by setting $\mathrm{NB} 2(N)=J$, the code then chooses the best (smallest) upper bound $W_{n}(t)_{\max }$ and the best (largest) lower bound $w_{n}(t)_{\min }$

$$
W_{n}(t)_{\min } \leq W_{n}(t) \leq W_{n}(t)_{\max }
$$


From Equations (36) and (39), if $J=N_{c}-n+1$, then

$$
\mathrm{w}_{\mathrm{n}}(t)_{\min }=\mathrm{w}_{\mathrm{n}}(t)_{\max } ; J=N_{c}-\mathrm{n}+I
$$

and the exact value of $w_{n}(t)$ is obtained, given by either $w_{n}(t)_{\min }$ or $\mathrm{w}_{\mathrm{n}}(\mathrm{t})_{\max }$

The best upper and lower bounds for $w_{n}(t), n=1, \ldots, N B M A X$, are then used in Equation (33) to obtain upper and lower bounds (brackets) for $\check{\mathrm{W}}_{0}^{(2)}(t)$.

$$
\begin{aligned}
& \tilde{W}_{0}^{(2)}(t) \leq w_{1}(t) \max \\
& \left.\tilde{\mathrm{w}}_{\mathrm{O}}^{(2)}(\mathrm{t}) \geq \mathrm{w}_{1} \dot{(t)}\right)_{\min }-\mathrm{w}_{2}(t)_{\max } \\
& \tilde{W}_{0}^{(2)}(t) \leq w_{1}(t)_{\max }-W_{2}(t)_{\min }+W_{3}(t)_{\max } \\
& \check{\mathrm{W}}_{\mathrm{O}}^{(2)}(t) \geq \mathrm{W}_{1}(t)_{\min }-\mathrm{W}_{2}(t)_{\max }+\mathrm{W}_{3}(t)_{\min }-\dot{\mathrm{W}}_{4}(t)_{\max } \\
& \text { etc. }
\end{aligned}
$$

This is done in the code until NBMAX terms are considered on the righthand side of the above equations [ie, until NBMAX upper and lower bounds are obtained for $\left.\check{W}_{0}^{(2)}(t)\right]$. From these various bounds for $\check{W}_{0}^{(2)}(t)$, the . best upper bound for $\check{W}_{0}^{(2)}(t)$ and best lower bound for $\check{W}_{0}^{(2)}(t)$ are chosen by the code.

$$
\check{W}_{0}^{(2)}(t)_{\min } \leq \check{W}_{0}^{(2)}(t) \leq \check{W}_{0}^{(2)}(t)_{\max }
$$

where $\check{W}_{0}^{(2)}(t)_{\min }$ and $\tilde{W}_{0}^{(2)}(t)_{\max }$ are the best lower and upper bound, respectively.

If one assigns the values

$$
\mathrm{NB} 2(N)=N_{C}-N+1 ; N=1, \ldots, \operatorname{NBMAX}
$$

and

$$
\operatorname{NBMAX}=\mathrm{N}_{\mathrm{C}}
$$


where $N_{c}$ is the total number of minimal cut sets, then both of the bounds for $\check{\mathrm{W}}_{0}^{(2)}(t)$ are identical in value and are equal to the exact value of $\tilde{w}_{0}^{(2)}(t)$.

$$
\begin{aligned}
\check{w}_{0}^{(2)}(t) & =\check{w}_{0}^{(2)}(t)_{\min } \\
& =\check{w}_{0}^{(2)}(t)_{\max }
\end{aligned}
$$

From these best bounds for $\tilde{\mathrm{W}}_{0}^{(2)}(t)$ and from the best bounds for $\check{W}_{0}^{(1)}(t)$ (Input Group 9), the best bounds for the system failure rate $W_{0}(t)$ are determined;

$$
W_{0}(t)_{\min } \leq w_{0}(t) \leq w_{0}(t)_{\max }
$$

wherc

$$
\mathrm{w}_{0}(t)_{\min }=\check{W}_{0}^{(1)}(t)_{\min } \cdot-\check{W}_{0}^{(2)}(t)_{\max }
$$

and

$$
W_{0}(t)_{\max }=\check{W}_{0}^{(1)}(t)_{\max }-\check{W}_{0}^{(2)}(t)_{\min } .
$$

Finally, from these best bounds for $W_{0}(t)$ and from the best bounds for $Q_{0}(t)$ (Input Group 9) the best bounds for the system failure intensity $\Lambda_{0}(t)$ are obtainer;

$$
\Lambda_{0}(t)_{\min } \leq \Lambda_{0}(t) \leq \Lambda_{0}(\tau)_{\max }
$$

where

$$
\Lambda_{0}(t)_{\min }=\frac{W_{0}(l)_{\min }}{1-Q_{0}(t)_{\min }}
$$

and

$$
\Lambda_{0}(t)_{\max }=\frac{W_{0}(t)_{\max }}{1-Q_{0}(t)_{\max }} .
$$

These bounds for $\Lambda_{0}(t)$ are obtained from the exact equation

$$
\Lambda_{0}(t)=\frac{W_{0}(t)}{1-Q_{0}(t)} .
$$


If the values of $\mathrm{NB2}(\mathrm{N})$ and NBMAX are assigned those in Equations (50) and (5I), then one of the best bounds for $W_{0}(t), Q_{0}(t)$, and $\Lambda_{0}(t)$ is the exact value

$$
\begin{array}{l|l}
Q_{0}(t)=Q_{0}(t)_{\min } & N_{c} \text { even } \\
W_{0}(t)=W_{0}(t)_{\min } & \begin{array}{l}
N_{B M A}=N_{c} \\
\Lambda_{0}(t)=\Lambda_{0}(t)_{\min }
\end{array}
\end{array}
$$

or

$$
\left.\begin{array}{rl}
Q_{0}(t)^{\prime} & =Q_{0}(t)_{\max } \\
W_{0}(t) & =W_{0}(t)_{\max } \\
\Lambda_{0}(t) & =\Lambda_{0}(t)_{\max }
\end{array}\right\} \begin{aligned}
& \mathrm{N}_{\mathrm{c}} \text { odd } \\
& \mathrm{NBMAX}=\mathrm{N}_{\mathrm{c}} \\
& \mathrm{NB} 2(\mathrm{~N})=\mathrm{N}_{\mathrm{c}}-\mathrm{N}+1
\end{aligned}
$$

Recommendations for values of NB2(N) are given on page 36.

\section{B. Minimal Path Sets Input}

If minimal path sets are used to obtain system reliability information, then IFAG2 must be set equal to 2 , NBMAX must be set equal to $N_{p}$ (the total number of minimal path sets), and the following values must be assigned to $\mathrm{NB} 2(\mathrm{~N})$,

$$
\operatorname{NB2}(\mathrm{N})=\mathrm{N}_{\mathrm{p}}-\mathrm{N}+1, \mathrm{~N}=1, \ldots, \mathrm{NBMAX}
$$

This is necessary, not only to give the exact values for $Q_{0}(t), \dot{W}_{0}(t)$, and $\Lambda_{0}(t)$, but also to give any reasonable values for these characteristics. The exact values for $Q_{0}(t), W_{0}(t)$, and $\Lambda_{0}(t)$ are then one of the best bounds determined by the program:

$$
\left.\begin{array}{l}
Q_{0}(t)=Q_{0}(t)_{\min } \\
W_{0}(t)=W_{0}(t)_{\min } \\
\Lambda_{0}(t)=\Lambda_{0}(t)_{\min }
\end{array}\right\} \begin{aligned}
& N_{p} \text { even } \\
& \text { NBMAX }=N_{p} \\
& N B 2(\mathbb{N})=N_{p}-N+1
\end{aligned}
$$

or 


$$
\begin{array}{l|l}
Q_{0}(t)=Q_{0}(t)_{\max } & \\
W_{0}(t)=W_{0}(t)_{\max } & N_{p} \text { odd } \\
\Lambda_{0}(t)=\Lambda_{0}(t)_{\max } & \operatorname{NBMAX}=N_{p} \\
& \operatorname{NB} 2(N)=N_{p}-N+1
\end{array}
$$

where $Q_{0}(t)_{\max }$, etc, are the bounds determined by the code.

Recommendations For Input Groups 6,9, and 10 If Minimal Cut Sets Are Used.

We discuss here the most efficient values to assign the variables of Input Group 6 (ISTOP), Input Group 9, and Input Group 10. A procedure of selection of values for these variables is given which results in accurate values being obtained for the system characteristics in a minimum of computer time. The user who merely wishes the summary of this discussion is referred to page 41 where the procedure is simply stated.

1. When to Compute the Exact Values for the System Characteristics $($ ISTOP $=2)$

If minimal path sets are used, the values to be used in Input Groups 6, 9, and 10 are given in the above discussions [Equation (64) or (65)] and are no problem. If minimal cut sets are used, then the values to be used for Input Group 9 and Input Group 10 must be chosen with some discretion. If the total number of minimal cut sets for the fault tree is less than approximately 10, then the exact values of $Q_{0}(t), W_{0}(t)$, and $\Lambda_{0}(t)$ can be obtained in relatively sinall computer time ( $\leqslant 1$ minute). These exact values are nhtained by aosigning the values

$$
\begin{aligned}
\text { ISTOP } & =2 \\
\operatorname{NBMAX} & =\mathrm{N}_{C} \\
\operatorname{IFAG2} & =2 \\
\operatorname{NB} 2(N) & =N_{C}-N+1 ; N=1, \ldots, N_{C}
\end{aligned}
$$


where $\mathbb{N}_{C}$ is the total number of minimal cut sets. The exact values of $Q_{0}(t), W_{0}(t)$, and $\Lambda_{0}(t)$ are then either the upper or lower bound, determined by the code, depending upon whether $\mathrm{N}_{\mathrm{c}}$ is even or odd;

$$
\left.\begin{array}{l}
Q_{0}(t)=Q_{0}(t)_{\min } \\
W_{0}(t)=W_{0}(t)_{\min } \\
\Lambda_{0}(t)=\Lambda_{0}(t)_{\min }
\end{array}\right\} N_{c} \text { even }
$$

or

$$
\left.\begin{array}{rl}
Q_{0}(t) & =Q_{0}(t)_{\max } \\
W_{0}(t) & =W_{0}(t)_{\max } \\
\Lambda_{0}(t) & =\Lambda_{0}(t)_{\max }
\end{array}\right\} N_{c} \text { odd } .
$$

2. The Accuracy of the Upper Bound Approximations (ISTOP = I)

If the total number of minimal cut sets is greater than 10, then attempting to obtain exact system answers becomes time consuming, and the bounding procedures used in the code are an efficient way of obtaining excellent approximations, or tight envelopes, for $Q_{0}(t), W_{0}(t)$, and $\Lambda_{0}(t)$. As stated in Input Group 6 , setting

$$
\text { ISTOP }=1
$$

and not obtaining results from bracketing (skipping Input Groups 9 and 10) will yield upper bounds for $Q_{0}(t), W_{0}(t)$, and $\Lambda_{0}(t)$, which are also excellent approximations to the true values. The upper bounds can furthermore be obtained in negligible computer time (fractions of a minute for 500 minimal cut sets) [a]. The upper bounds are again

$$
Q_{0}(t) \leq 1-\prod_{i=1}^{N_{c}}\left[1-\check{Q}_{i}(t)\right]
$$

\footnotetext{
[a] As stated in Input Group 7, the number of time points used does not significantly affect the computer time.
} 


$$
\mathrm{w}_{0}(t) \leq \sum_{i=1}^{N_{C}} \dot{W}_{i}(t)
$$

and

$$
\Lambda_{0}(t) \leq \frac{\sum_{i=1}^{N_{C}} \tilde{w}_{i}(t)}{\prod_{i=1}^{N_{C}}\left[1-\check{Q}_{i}(t)\right]}
$$

where the upper bounds the code yields are on the right-hand sides of these equations. $\check{Q}_{j}(t)$ is the failed probability for the $i^{\text {th }}$ minimal cut set, $\tilde{W}_{i}(t)$ is the $i^{\text {th }}$ minimal cut set failure rate, and $N_{c}$ is the total number of minimal cut sets.

The upper bound for $Q_{O}(t)$, Equation (1), becomes an exact value for $Q_{0}(t)$ if the minimal cut sets have no components in common (ie, the minimal cut sets are independent);

$$
\begin{aligned}
& Q_{O}(t)=1-\prod_{i=\tau}^{N_{c}}\left[1-\check{Q}_{i}(t)\right] ; \text { No components } \\
& \text { or more mini- } \\
& \text { mal cut sets. }
\end{aligned}
$$

This general upper bound is therefore obtained assuming independence of the minimal cut sets. Restating Equation $(10), Q_{0}(t)$ is exactly

$$
Q_{0}(t)=Q_{1}(t)-Q_{2}(t)+Q_{3}(t)-\cdots
$$

where again

$$
\begin{aligned}
Q_{n}(t)= & \text { the contribution from exactly } n \\
& \text { winlmal cut sets simultaneously } \\
& \text { being in a failed state at time } \\
& t .
\end{aligned}
$$

From Equation (10), $Q_{0}(t)$ may be expressed as

$$
Q_{0}(t)=\dot{Q}_{1}(t)-Q_{\geq 2}(t)
$$


where the second term on the right-hand side of Equation (73) is the contribution from two or more minimal cut sets being simultaneously in a failed state at $t$. Now $[2]$

where

$$
Q_{\geq 2}(t) \sim N_{m} q_{a}(t) Q_{1}(t)
$$

and

$$
\begin{aligned}
\mathrm{q}_{\mathrm{a}}(\mathrm{t})= & \text { the average component failed } \\
& \text { probability (a simple average } \\
& \text { over all the components of } \\
& \text { the fault tree) }
\end{aligned}
$$

$$
\begin{aligned}
\mathbb{N}_{m}= & \text { the number of smallest cut } \\
& \text { sets (those having } m \text { compo- } \\
& \text { nents, where } m \text { is the smallest } \\
& \text { number in a minimal cut set). }
\end{aligned}
$$

The symbol " $\sim$ " denotes "is of the order". Therefore, when $q_{a}(t)<<1$, which is the general case, $Q_{\geq 2}(t)$ is negligible compared to $Q_{1}(t)^{[a]}$.

In the upper bound for $Q_{0}(t)$, given by Equation $(1)$, the term $Q_{1}(t)$ is computed exactly. The term $Q_{\geq 2}(t)$, which is generally negligible anyhow, is not neglected but is approximated by an independence assumption. The terms $Q_{2}(t), Q_{3}(t)$, etc, of $Q_{\geq 2}(t)$ [Equation (10)] are approximated. hy the products of the individual minimal cut set failed probabilities involved in the particular combinations; from Equation (13) $Q_{n}(t)$ is approximated by the expression

$$
Q_{n}(t) \cong \sum_{i_{1} \cdots i_{n}} \check{Q}_{i_{1}}(t) \check{Q}_{i_{2}}(t) \ldots \check{Q}_{i_{n}}(t) ; \text { independence } . .
$$

where $\check{Q}_{\dot{I}_{1}}(t)$, etc, are the individual minimal cut set failed probabilities. This approximation of $Q_{\geq 2}(t)$ can be shown to always give a better approximation for $Q_{O}(t)$, as compared to neglecting

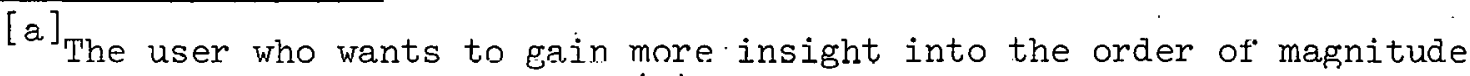
of the various terms for $Q_{0}(t)$, etc, should see the report Analysis of Fault Trees by Kinetic Tree Theory [2].
} 
$Q_{\geq 2}(t)$ [though the two approximations would be very close in value because of the negligibility of $\left.Q_{\geq 2}(t)\right]$. Therefore, because $Q_{1}(t)$ is computed exactly, and, in a minor significance, because $Q_{\geq 2}(t)$ is not neglected but is approximated, the upper bound obtained for $Q_{0}(t)$, Equation (1), is also an excellent approximation to its exact value.

The upper bound obtained for the system failure rate, Equation (2), obtained by setting ISTOP $=1$ is merely the sum of the individual minimal cut set failure rates. [This is the-first term on the righthand side of Equation (15).] From Equations (14) and (15) we may express the exact failure rate as

$$
w_{0}(t)=w_{0}^{u}(t)-\left\{\check{W}_{\geq 2}^{(-1)}(t)+\check{W}_{0}^{(2)}(t)\right\}
$$

where $w_{0}^{\mathrm{u}}(t)$ is the upper bound given by Equation (2)

$$
w_{o}^{u}(t)=\sum_{i=1}^{N_{c}} \check{W}_{i}(t)
$$

and

$$
\begin{aligned}
\check{\mathrm{W}}_{\geq 2}^{(1)}(t)= & \text { the contribution to } \mathrm{W}_{0}(t) \text { from } \\
& \text { two or more miniman riut sets } \\
& \text { simutaneously friting per unit } \\
& \text { time at time } t .
\end{aligned}
$$

$\check{W}_{0}^{(2)}(t)$ is the "correction term" discussed in Input Group 10. Considering $W_{o}^{u}(t)$ not only as an upper bound but also as an approximation for $w_{0}(t)$ involves neglecting the two terms within the brackets in Equation (78).

Now [2]

$$
\check{W}_{\geq 2}^{(1)}(t) \sim q_{a}(t) w_{0}^{u}(t)
$$

and

$$
\check{w}_{0}^{(2)}(t) \sim q_{a}(t) w_{o}^{u}(t) . .
$$


Thus, when $q_{a}(t)<1$, which $\overline{\text { as }}$ stated is the general case, these two terms are negligible and the upper bound $\mathrm{w}_{0}^{\mathrm{u}}(t)$ given by Equation (2) for $W_{0}(t)$ is therefore also an excellent approximation for its true value.

The upper bound for the system failure intensity $\Lambda_{0}(t)$, given by Equation (3) and obtained when ISTOP $=1$, can be simply expressed as

$$
\Lambda_{0}(t) \leq \frac{W_{0}^{u}(t)}{1-Q_{0}^{u}(t)}
$$

where $w_{0}^{u}(t)$ is the upper bound for $W_{0}(t)$ given by Equation (2) and $Q_{O}^{U}(t)$ is the upper bound for $Q_{0}(t)$ given by Equation (1). The exact value for $\Lambda_{0}(t)$ is given by the expression

$$
\Lambda_{0}(t)=\frac{W_{0}(t)}{1-Q_{0}(t)} .
$$

'l'he upper bound for $\Lambda_{0}(t)$ is therefore obtained by replacing $W_{0}(t)$ and $Q_{O}(t)$ by their respective upper bounds. Because $W_{0}^{u}(t)$ and $Q_{0}^{u}(t)$ are excellent approximations to the respective true values, the upper bound for $\Lambda_{0}(t)$, Equation (3), is therefore also an excellent approximation to its true value.

By setting ISTOP $=1$, the upper bounds yielded for $Q_{0}(t), W_{0}(t)$, and $\Lambda_{0}(t)$ are thus in general also excellent approximations to their true values ${ }^{[a]}$. From the previous discussion, the upper bounds are generally within three significant figures of the true values. The upper bounds yielded for the system integral characteristics [Equations (7) and (8)], which use the upper bounds for $W_{0}(t)$ and $\Lambda_{0}(t)$, are therefore likewise excellent approximations. In a converse manner,

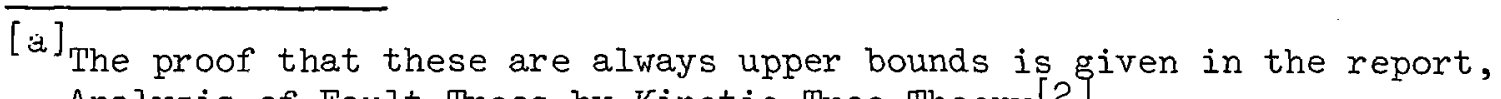
Analysis of Fault Trees by Kinetic Tree Theory [2]. 
the approximations obtained for $Q_{n}(t), W_{0}(t), \Lambda_{n}(t)$, and the integral characteristics are also upper bounds of their true values. This is quite important since the values are thus always conservative and result in conservative estimates for system failure phenomena. Though arguments have been given to validate the accuracy of these upper bounds, an appreciation of their accuracy and value (being upper bounds) is perhaps gained only through application. As stated in Input Group 6, because of the little computer time needed to obtain these upper bounds, if system results are obtained by use of bracketing (setting ISTOP = 2), these upper bounds, which are only obtained if ISTOP $=1$, are output as additional information. After a few comparisons are made with the exact results and after an understanding is gained of the assumptions involved in these upper bounds; it is expected that the user will then only obtain these upper bounds in the majority of cases for which he wishes system reliability characteristics.

3. Recommendations for the Use of Bracketing (ISTOP $=2$ )

From Input Group 9 and 10 , the exact values of $Q_{0}(t)$ and $W_{0}(t)$ dre glven by the equations

$$
\begin{gathered}
Q_{0}(t)=Q_{1}(t)-Q_{2}(t)+Q_{3}(t) \\
-\ldots+(-1)^{N_{c}-1} Q_{N_{C}}(t) \\
W_{0}(t)=\breve{W}_{u}^{(1)}(t)-\breve{W}_{u}^{(2)}\left(t_{1}\right) \\
\check{W}_{n}^{(1)}(t)=W_{1}^{(1)}(t)-W_{p}^{(1)}(t)+W_{3}^{(1)}(t) \\
-\ldots+(-1)^{\mathbb{N}_{c}-1} W_{\mathbb{N}_{c}}^{(1)}(t)
\end{gathered}
$$




$$
\check{W}_{0}^{(2)}(t)=W_{1}(t)-W_{2}(t)+W_{3}(t)-\ldots+(-1)^{N_{C}-1} W_{N_{C}}(t)
$$

and

$$
\begin{gathered}
w_{n}(t)=W_{n}^{I}(t)-W_{n}^{2}(t)+w_{n}^{3}(t) \\
-\ldots(-1)^{N_{c}-1} w_{n}^{N_{c}}(t), n=1, \ldots, N_{c} .
\end{gathered}
$$

$\Lambda_{0}(t)$, the system failure intensity, is then given by the equation

$$
\Lambda_{0}(t)=\frac{W_{0}(t)}{1-Q_{0}(t)} .
$$

NBMAX in Input Group 9 denotes the number of terms to consider on the right-hand sides of Equations (10) and (15) for $Q_{0}(t)$ and $\check{W}_{0}^{(1)}(t)$, respectively. If IFAG2 $=2$, it is also the number of terms to consider on the right-hand side of Equation (33) for $\tilde{w}_{0}^{(2)}(t)$. If lower bounds are desired in addition to upper bounds, IFAG2 must be set equal to 2. Since this is generally desired when bracketing is done, we will assume IFAG2 $=2$.

Setting NBMAX equal to 2 will yield one upper bound and one lower bound for $Q_{0}(t), \check{W}_{0}^{(1)}(t)$, and $\check{W}_{0}^{(2)}(t)$. Since they are the only bounds, they will be the best bounds used by the code. Setting NBMAX $=3$ will yield two upper bounds and one lower bound. The best upper bound will be chosen and the one lower bound will be used. Setting NBMAX = 4 will yield two upper bounds and two lower bounds. The best upper bound and best lower bound will be used. Setting NBMAX $=5$ will yield three upper bounds and two lower bounds, and so forth. Because of this behavior NBMAX should be generally set equal to an even number in order to obtain an equal number of upper and lower bounds.

It is recommended that if bracketing is desired, NBMAX be set equal to 2;

$$
\operatorname{NBMAX}=2
$$


Each succeeding term on the right-hand sides of Equations (10), (15), and (33) is generally of the order of $q_{a}(t)$ times the previous term where $q_{a}(t)$ is an average component failed probability [Equation (75)]. Hence, the upper and lower bounds obtained will have a fractional difference of $\mathrm{q}_{\mathrm{a}}(t)$, and the true values will be very tightly enveloped, generally within three significant figures. Moreover, for NBMAX $=2$, these bounds can be obtained in moderate computer time--on the order of several minutes $360 / 75$ time for 500 minimal cut sets ${ }^{[a]}$.

For those instances when these bounds are not close enough, which will be unusual, NBMAX could then be set, equal to 4, etc. However, computer times for a larger number of minimal. cut sets (100 or more) will then increase to orders of an hour or more $360 / 75$ time. For these instances when setting NBMAX $=2$ does not yield close enough upper and lower bounds, and when the user is not content with the upper bounds described in the previous section, it is recommended that the less important cut sets (those with a larger number of components) be neglected or that the minimal cut sets be regrouped by set theory rearrangement. The neglection of the larger number component minimal cut sets (for example neglecting the minimal cut sets with three or more components if minimal cut sets of one component are present) will cause negligible error in the system results and in many cases will allow the user to run KITT-1 (with only these minimal cut sets of a smaller number of components) in very small computer time ${ }^{[b]}$. The

\footnotetext{
[a]Again, the number of time points used does not significantly affect. the computer time. 
use of KITT-I with these minimal cut sets of a smaller number of components should be the same as before, following the same procedures as recommended herein. Grouping of the minimal cut sets has the same results, enabling accurate system results to be obtained in little computer time.

Assuming NBMAX has been set equal to 2 and IFAG2 $=2$, input values for $N B 2(N)$ in Input Group 10 must then be decided upon. NB2(n), where we have replaced the index $\mathbb{N}$ by $n$, is the number of terms to consider on the right-hand side of Equation (36) to obtain a best upper and best lower bound for $W_{n}(t)$. NBMAX sets the number of terms on the righthand side of Equation (33) to consider for $\check{W}_{0}^{(2)}(t)$. However, each of these terms, $W_{1}(t), W_{2}(t)$, etc, is not exactly computed as with $Q_{0}(t)$ and $\grave{W}_{0}^{(1)}(t)$, but is bracketed. $N B 2(n)$ prescribes how tight this bracketing, or cnveloping, will be for each term.

From Equation (14) to obtain an upper and lower bound for $w_{0}(t)$ an upper and lower bound are needed for $\check{W}_{0}^{(2)}(t)$. As stated previously, $\tilde{W}_{0}^{(2)}(t)$ is of the order of $q_{a}(t)$ times $\tilde{W}_{0}^{(l)}(t)$ and is therefore generally much smaller than $\check{W}_{0}^{(1)}(t)$. Therefore an upper bound need only be computed for $\tilde{W}_{0}^{(2)}(t)$, and a lower bound of zero, preset by the code, may be used as the best lower bound [a]. We have assumed NBMAX has been set equal to 2, and therefore from Equation (33)

$$
\check{\mathrm{W}}_{0}^{(2)}(\mathrm{t})_{\max }=\mathrm{w}_{1}(\mathrm{t})_{\max }
$$

where $W_{1}(t)_{\max }$ is the best upper bound for $W_{1}(t)$ chosen by the code

\footnotetext{
[a] Before computation of the best bounds for the system differential characteristics, the code initializes the best lower bounds at 0.0 and the best. upper hnindis a.t. $1.0^{10}$, the upper bound value being an arbitrarily large number.
} 
from the NB2(I) upper and lower bounds for it. $W_{1}(t)_{\max }$ will he the only, and hence best, upper bound for $\check{\mathrm{w}}_{0}^{(2)}(t)$ since NBMAX $=2$ [NBMAX $=3$ being the smallest value for more than one upper bound for $\left.\check{W}_{0}^{(2)}(t)\right]$.

Setting $\mathbb{N B 2}(I)=1$ gives one upper bound for $W_{1}(t)$, setting $\operatorname{NB2}(1)=2$ gives one upper and one lower bound for $w_{1}(t)$, etc. Now, each succeeding term on the right-hand side of Equation (36) for $w_{n}(t)$ is generally of the order of $q_{a}(t)$ times the preceding term. Therefore, the first term $W_{l}^{l}(t)$ is an excellent approximation for $W_{l}(t)$, and moreover, this first term wi.l. he used as the beot upper bound for $W_{1}(t)$ if we set $\operatorname{NB2}(1)=1$. Thus, the minimum values to assign $\operatorname{NB2}(n)$, $\mathrm{n}=1,2$, are

$$
\operatorname{NB} 2(1)=1
$$

and

$$
\mathrm{NB} 2(2)=0
$$

where we set $\mathbb{N B 2}(2)=0$ since we do not compute $W_{2}(t)$.

If a nonzero lower bound, is desired for $\tilde{W}_{0}^{(2)}(t)$ then from Equations (33) and (36)

$$
\check{W}_{0}^{(2)}(t)_{\max }=W_{1}(t) \max
$$

and

$$
\check{\mathrm{W}}_{0}^{(2)}(t)_{\min }=\mathrm{w}_{1}(t)_{\min }-\mathrm{w}_{2}(t)_{\max } .
$$

Since NBMAX $=2$, one upper and one lower bound can be obtained for $\check{W}_{0}^{(2)}(t)$ and they will be the best bounds used by the code. We therefore need at minimum nne ppper and one lowcr bound for $W_{1}(l)$ and one upper bound for $W_{2}(t)$. These bounds will be obtained by setting

$$
\mathrm{NB} 2(1)=?
$$

and

$$
\operatorname{NB2}(2)=1
$$


Setting $\operatorname{NB2}(1)=2$ and $\mathrm{NB} 2(2)=1$, with $\mathbb{N B M A X}=2$, will in general yield an upper and lower bound for $w_{0}(t)$ which are closer to each other than the upper and lower bound obtained by setting $\mathrm{NB} 2(1)=1$ and $\mathrm{NB} 2(2)$ $=0$. However, with $\operatorname{NB2}(1)=1$ and $N B 2(2)=0$, the upper and Iower bounds obtained for $W_{0}(t)$ will generally differ in the third or fourth significant figure. The value of $w_{0}(t)$ will therefore be accurately known to within three significant figures, which for most reliability analyses is of sufficient accuracy. Moreover, the computer time needed for $\operatorname{NB2}(1)=1$ and $\operatorname{NB2}(2)=0$ is on the order of a few minutes for 500 minimal cut sets, while that needed for $\mathrm{NB} 2(1)=2$ and $\mathrm{NB} 2(2)=1$ is on the order of an hour. Therefore, with NBMAX $=2$ and IFAG2 $=2$, it is recommended that onc inputs the values

$$
\mathrm{NB} 2(1)=1
$$

and

$$
\operatorname{NB2}(2)=0
$$

As recommended for NBMAX, for those unusual cases where $\mathrm{NB} 2(1)=$ 1 and NB2(2) = 0 do not yield sufficiently tight bounds, and where the upper bounds discussed in Section 2 are not deemed satisfactory, it is recommended that either the less important minimal cut sets be neglected and the procedures be reperformed as recommended herein, or that regrouping of the minimal cut sets be done.

\section{Recommendation Summary}

If the total number of minimal cut sets $\mathrm{N}_{\mathrm{C}}$ is less than approximately 10, the exact values for the system characteristics can be obtained in several minutes computer time by setting; 


$$
\begin{aligned}
& \text { ISTOP }=2 \\
& \text { NBMAX }=\mathrm{N}_{\mathrm{C}} \\
& \text { IFAG2 }=2
\end{aligned}
$$

and

$$
\mathrm{NB} 2(\mathrm{~N})=\mathrm{N}_{\mathrm{C}}-\mathrm{N}+1 ; \mathrm{N}=1, \mathrm{~N}_{\mathrm{C}}
$$

The total number of time points used does not significantly affect this computer time needed. Depending upon whether $\mathbb{N}_{c}$ is even or odd, the exact values for the system characteristics are given either by Equation (61) or by Equation (62) on page 29 ;

If the total number of minimal cut sets is greater than approximately 10, system-characteristics are most efficiently obtained either by using their upper bound approximations or by bracketing. Setting ISTOP $=1$ and skipping Input Groups 9 and 10 will yield upper bounds for the system characteristics which are accurate (generally to within three significant figures) and which can be obtained in negligible computer time, regardless of the number of time points used.

If both upper and lower bounds are desired for the systam characteristics, then set

$$
\begin{aligned}
\text { ISTOP } & =2 \\
\text { NBMAX } & =2 \\
\text { IFAG2 } & =2 \\
\operatorname{NB2}(1) & =1
\end{aligned}
$$

and

$$
\operatorname{NB} 2(\Omega)-0 \cdot
$$

This will result in the upper and lower bounds being very close to one another, and the system chararteristiss will be obtaincd to withili generally three significant figures. The computer time will furthermore be moderate for any number of time points used, on the order of 
a few minutes. For those instances when it is deemed that neither of these procedures yields satisfactory results, then one should neglect the lesser important minimal cut sets or one should regroup the minimal cut sets.

\section{Input Group 11}

Number of Cards: 1

Format: II0

Input Data:. IPATH

IPATH is a flag and has either a value of 1 or 2 . If minimal cut sets are read in, then IPATH is set equal to 1 . If minimal path sets are read in, then IFATH is set equal to 2 .

\section{Input Group 12}

Number of Cards: 1

Format: II0

Input Data: NCUT ( $1 \leq$ NCUT $\leq 500)$

NCUT is the total number of unique minimal cut sets (IPATH = 1) or minimal path sets (IPATH $=2$ ) input to obtain system reliability characteristics.

Read In The Following Input Group (Input Group 13) For Each Minimal Cut

Or Path Set. Repeat The Input Group For The NCUT Total Number of Sets. 


\section{Input Group 13}

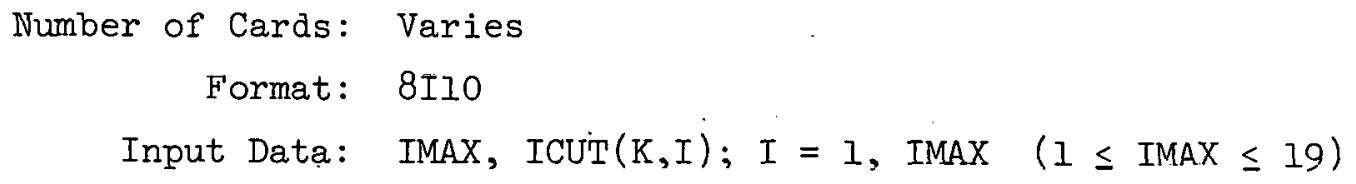

For NPROB $=1$ (Input Group 2) There Are No More Cards To Be Read In. If IVKÜ $; 1$, Kead In Input Groups 14, 15, 16, And 17 For Each of The (NPROB - 1) Different Parameter Runs. The Four Input Groups Are Repeated, But With Different Values, For The (NPROB - 1) Different Parameter Runs.

\section{Input Group 14 (New Parameter Run)}

Number of Cards: 1

Format: $\quad$ I10

Input Data: NLAM

NLAM is the number of components and inhibit conditions which have 
different failure intensities $\lambda[\mathrm{XLMDA}(I)]$ for the new parameter run. The values XLMDA(I) are with reference to Input Group 4.

If NLAM Equals 0, Skip Input Groups 15A And 15B And Go To Input Group 16. If NLAM Equals NCOMP, The Total Number of Components And Inhibit Conditions (Input Group 3), Read In Input Group 15A And Then Go To Input Group 16. If NLAM Is Less Than NCOMP, Read In Input Group 15B And Then Go To Input Group $\underline{\underline{16 .}}$

Input Group 15A (New Parameter Run)

Number of Cards: Varies

Format: $8 \mathrm{~F} 10.0$

Input Data: XLMDA(I); I = I, NCOMP

This Input Group is skipped if NLAM

is zero or is less than NCOMP.

$X L M D A(I)$ are the new failure intensities $\lambda$ (per hour) for the parameter run. These are different values for Input Group 4 (with the indices used for the components and inhibit conditions remaining unchanged for the new parameter runs). The interpretation for a nonpositive XLMDA(I) is the same as for Input Group 4.

Input Group 15B (New Parameter Run)

Number of Cards: Varies

Format: $4(110, F 10.0)$

Input Data: $\operatorname{IND}(K), \operatorname{XLMDA}(\operatorname{IND}(K)) ; K=1, N L A M$ 
This Input Group is skipped if NLAM is zero or is equal to NCOMP.

$\operatorname{IND}(K)$ is the index of the component or inhibit condition having a new failure intensity and XIMDA $[\operatorname{IND}(K)]$ is the corresponding new failure intensity (per hour). Each card contains the values $i_{k}, \lambda\left(i_{k}\right), i_{k+1}$, $\lambda\left(i_{k+1}\right), i_{k+2}, \lambda\left(i_{k+2}\right), i_{k+3}, \lambda\left(i_{k+3}\right)$ where $i_{k}, i_{k+1}$, etc, are the indices of the components or inhibit conditions having different failure intensities (the components and inhibit conditions have the same indices for all parameter runs, ie, the indices are the same as for Input Group 4), and the $\lambda\left(i_{k}\right), \lambda\left(i_{k+1}\right)$, etc, are the corresponding new failure intensities. There are four pairs of values $\left[i_{k}, \lambda\left(i_{k}\right)\right]$ to a card until. the NLAM pairs of values are read in. The interpretation for a nonpositive $\lambda\left(i_{k}\right)$ is the same as for Input Group 4. Those failure intensities not changed retain the same values as used for the preceding parameter run (ie, those values of' $\lambda$ not chänged t'or parameter run 2 remain those values used for parameter run 1; for parameter run 3, the unchanged values remain thooc valucs used for parameter run'2, etr).

\section{Input Group 16 (New Parameter Run)}

Number of Cards: 1

Format: $I 10$

Input Data: NTAU

NTAU is the number of components and inhibit conditions having different repair times [TAU(I)] for the new parameter run. The values, TAU(I), are with reference to Input Group 5. 
If NTAU Equals 0, There Is No Further Data To Input For This Parameter

Run. Go Back To Input Group 14 For Additional Parameter Runs. If NTAU

Equals NCOMP, Read In Input Group 17A As the Last Input Group For This

Parameter Run and Then Return To Input Group 14 For Additional Parameter

Runs. If NTAU Is Less Than NCOMP, Read In Input Group 17B As the Last

Input Group For This Parameter Run And Then Return To Input Group 14 For

Additional Parameter Runs.

Input Group 17A (New Parameter Run)

Number of Cards: Varies

Format: $8 \mathrm{~F} 10.0$

Input Data: TAU(I); I = I, NCOMP

This Input Group is skipped if NTAU is zero or is less than NCOMP.

TAU(I) are the new repair times $\tau$ (hours) for the parameter run. These are different values for Input Group 5 (with the indices for the components and inhibit conditions remaining unchanged for the new parameter runs). The interpretation for a nonpositive $\mathrm{TAU}(I)$ is the same as for Input Group 5.

Input Group i7B (New Parameter. Run)

Number of Cards: Varies

Format: $\quad 4(\mathrm{IIO}, \mathrm{F} 10.0)$

Input Data: $\operatorname{IND}(K), \operatorname{TAU}(\operatorname{IND}(K)) ; K=1$, NTAU 
This Input Group is skipped if NTAU is zero or equals NCOMP.

$\operatorname{IND}(K)$ is the index of the component or inhihit condition having a new repair time, and $\operatorname{TAU}[\operatorname{IND}(K)]$ is the corresponding new repair time (hours). Each card of this input group contains the values $j_{k}, \tau\left(f_{k}\right)$, $j_{k+1}, \tau\left(j_{k+1}\right), j_{k+2}, \tau\left(j_{k+2}\right), j_{k+3}, \tau\left(j_{k+3}\right)$. The values $j_{k}, j_{k+1}$, etc, are the indices of the components or inhibit conditions having different repair times and $\tau\left(j_{k}\right), \tau\left(j_{k+1}\right)$, etc, are the corresponding different times. There are four pairs of values $\left[j_{k}, \tau\left(j_{k}\right)\right]$ to a card until the NTAU pairs of values are read in. The interpretation for a nonpositive $\tau\left(j_{k}\right)$ is the same as for Input Group 5. Like Input Group 15B, the repair times not changed for the new parameter run retain their values as used in the preceding parameter run.

\section{FORMAT OF OUTPUT FROM KITT-I}

Section 11 dcscribed the reliabillty characteristics nht.ainer by KITT-1. This section describes the formats by which KITT-I prinls out these characteristics. The user will best enter thïs discussion if he realizes the general structure of the output formats for the reliability quantities :

1. Any quantity with the symbol " $Q$ " is a failed probability,

2. Any quantity with the symbol "W" is a failure rate.

3. Any quantity with the symbol " $L$ " is a f'ailure intensity (lambda).

4. The title "WSUM" denotes the integral of the failure rate $W$.

5. The title "FSUM" denotes the quantity $1-\exp \left[-\int_{0}^{t} \Lambda\left(t^{\prime}\right) d t^{\prime}\right]$
where $\Lambda\left(t^{\prime}\right)$ is a failure intensity. 
This general naming procedure is used for the output of component characteristics, minimal cut or path set characteristics, and for the system characteristics. With this general naming procedure in mind, the user should find the following discussions of the output formats more uniform in structure.

The first block of output from KITT-1 is a printout of the values read in for the variables of Input Group 1 through Input Group 13. In this first block of output, all the input read into the program is printed out, with the exception of Input Groups 14 through 17, which are the different values for the component $\lambda$ 's and $\tau$ 's for the different parameter runs. The $\lambda^{\prime} s$ and $\tau$ 's printed out in this first block of output are therefore only those for the first parameter run (ie, the values for Input Groups 4 and 5). Using these first parameter run $\lambda$ 's and $\tau$ 's, reliability characteristics are then printed out in succeeding blocks of output. If there is more than one parameter run (NPROB $>1$ ), the $\lambda$ 's and $\tau$ 's for the new parameter run (Input Groups 14 through 17) are printed out on a new page. This is then followed by a printout of the reliability characteristics obtained using this new set of $\lambda$ 's and $\tau$ 's. This form of printout, the new set of $\lambda$ 's and $\tau$ 's followed by the characteristics obtained, is repeated for as many parameter runs as were studied.

The format of printout for the reliability characteristics for the succeeding parameter runs is the same as the format for the first parameter run, and we will therefore only describe the format of output for. the first parameter run.

As stated, the first block of output from KITT-1 is a printout of the variables of Input Groups 1 through 13. This block of output is selfexplanatory, with the name of the variable (as used in the previous input 
description) and its definition being printed out along with its value. The last output of this first block is titled "SET INFORMATION" and is printed out in the form

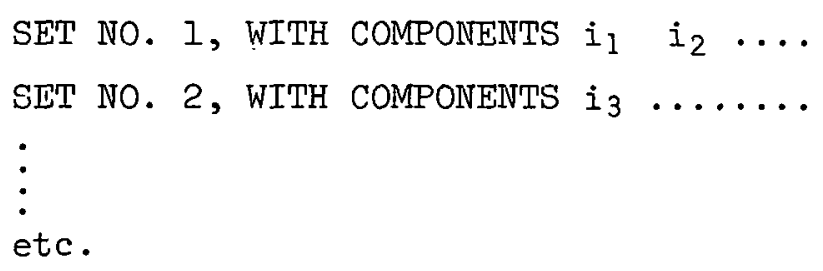

This is a printout of the index, or number, of the minimal cut or path set along with the indices of the components (or inhibit conditions) in the set (Input Group 13). The minimal cut sets or minimal path sets are indexed by the code in the nrier in which thoy arc rcad in fü Inpul Group 13. These indices will be used in subsequent output to identify the individual sets.

Immediately following this first block of output are succeeding blocks of output containing the reliability characteristics obtained by the program. The block of output entitled "COMPONENT AND INHIBIT INFORMATION", beginning on a new page, is the first of these outputs and contains the reliability characteristics obtained for each component. The index (number) of the component is first given, followed by its reliability characteristics. The first column of output is the time and each succeeding column is a particular component characteristic for the times given in the first column. (One should therefore read row-wise to obtain all the component characteristics for a particular time.) The conponent characteristics printed out have been described a.t the beginning of this report. Table 10 on page 55 gives the transiation from the program output (the headings of each column) to the symbols used on page $2^{[a]}$.

[a]All the translation tables are given immediately after this section. 
Also in the output group, "COMPONENT AND INHIBIT INFORMATION", is the inhibit condition information. The output for the inhibit condition is merely a printout of the data read in, with the index and occurrence probability being output. Descriptive labelling is used, and the output format is self-explanatory.

On a new page following the component characteristics and inhibit information is a block of output entitled "MINIMAL SET INFORMATION". This block of output contains the reliability characteristics/for each minimal cut set or path set. The output is of the same format as the previous component output; the set index is first given, followed by its reliability characteristics which are in columns. The set characteristics printed out have been described on pages 3 and 5 . Table 11, at the end of this section, gives the translation from the program output (column headings) to the symbols used on page 3 , if minimal cut sets are used. Table 12 gives the same translation if minimal path sets are used.

Following the minimal set characteristics, on a new page, is a block of data which is present only if minimal cut sets are used. 'l'his block of data, entitled "SYSTEM INFORMATION - UPPER BOUNDS", contains the upper bound approximations for the system characteristics which were discussed in Input Group 6. The first group of output in.this block of data is subtitled "DIFFERENTIAL CHARACTERISTICS - UPPER BOUNDS" and contains the upper bounds for the differential system characteristics. Immediately following is a second group of output subtitled "INTEGRAL CHARACTERISTICS UPPER BOUNDS" which contains the upper bounds for the integral system characteristics. Table 13 gives the translation from the program output (column headings) to the symbols used in Input Group 6.

If ISTOP $=1$ Group 6), the output for the first parameter run is completed and the above described output will be repeated for the 
succeeding parameter runs, if there are any. If ISTOP $=2$, there will be one further block of output which contains the best brackets obtained for the system characteristics, described in Input Groups 9 and 10. This block of output is titled "SYSTEM INFORMATION - BEST BRACKETS" and has several groups of output. The first group of output is subtitled "DIFFERENTIAL CHARACTERISTICS - BEST BRACKETS" and contains the best upper and lower bounds determined by the code for the differential system characteristics. The format of output is analogous to the previous output, with a particular system characteristic being in a particular solumn (a row correepondo to a given time). Table 14 at the end of this section gives the translation from program output to those symbols used in Input Groups 9 and 10.

On a new page immediately following the first group of system bracketed output is the second group, entitled "FAILURE RATE CONTRIBUTIONS". This output will generally be of minor concern to the user since it gives the contributions to the system failure rate $w_{0}(t)$ from which the best bounds are obtained for $W_{0}(t)$. If minimal cut sets are used then from Input Group 9,

$$
W_{0}(t)=\tilde{W}_{0}^{(1)}(t)-\tilde{W}_{0}^{(2)}(t)
$$

In this case, the output contains the best bounds for $\tilde{W}_{0}^{(1)}(t)$ and $\check{W}_{0}^{(2)}(t)$. If minimal path sets are used,

$$
W_{0}(t)=\hat{W}_{0}^{(1)}(t)-\hat{W}_{0}^{(2)}(t)
$$

and the output then contains the hest hounds for $\hat{W}_{0}^{(1)}(t)$ and $\hat{W}_{0}^{(2)}(t)$. Tables 15 and 16 give the translation from the program output to the symbols used in Input Groups 9 and 10.

The next output group entitled, "DIFFERENTIAL CHARCTERISTICS-LAST BRACKETS", is of use only if exact values for the system characteristics 
are obtained [ie, Equations (61) and (62) or Equations (64) and (65)]. If the system characteristics are merely bracketed, then the user should not refer to this output.group. As stated for Input Groups 9 and 10, when exact values are computed, then one of the best bounds of the output group "DIFFERENTIAL CHARACTERISTICS - BEST BRACKETS" (Table 14 ) is the exact value for the particular system characteristics. Which bound is the exact value depends upon whether the number of sets is odd or even. To aid the user in determining whether the upper bound or lower bound is the exact value, this output group, "DIFFERENTIAL CHARACTERISTICS - LAST BRACKETS", printed out by KITT-1, contains the exact values for the system characteristics, when these exact values are computed. The user may compare these values with the best bounds to determine whether the upper bound or lower bound is the exact value ${ }^{[a]}$. When exact values are computed, then QIAST is the exact value $Q_{0}(t)$ and will be exactly equal to one of the best bounds for $Q_{0}(t)$ in the output group, "DIFFERENTIAL CHARACTERISTICS - BEST BRACKETS". If minimal cut sets are used, then WILAST is the exact value for $\check{W}_{0}^{(1)}(t)$ and will be equal to one of the best bounds for $\tilde{W}_{0}^{(l)}(t)$. For both $Q_{0}(t)$ and $\check{W}_{0}^{(l)}(t)$, the same bounds will be the exact values. If the upper bounds of $\tilde{W}_{0}(1)(t)$ and $Q_{0}(t)$ are their exact values, then the upper bounds for $\underline{W}_{0}(t)$ and $\Lambda_{0}(t)$ are also, respectively, their exact values. If the lower bounds of $\tilde{W}_{O}(l)(t)$ and $Q(t)$ are their exact values, then the lower bounds for $W_{0}(t)$ and $\Lambda_{0}(t)$ are also, respectively, their exact values. In addition, if minimal cut sets are used, W2MIN - LAST or W2MAX - LAST is the exact value for $\tilde{W}_{0}^{(2)}(t)$ and may be checked against one of its bounds. (If the exact values are computed, then W2MAX - LAST = W2MIN - LAST for minimal cut sets and for minimal path sets.) If minimal path sets are [a] One may, of course, also use the criteria given in Input Groups 9 and 10; ie, Equations (61) and (62) or Equations (64) and (65). 
used, then WILAST is the exact value for $\hat{W}_{u}^{(1)}(t)$ and WPMAX - LAST (or W2MIN - LAST) is the exact value for $\hat{W}_{0}^{(2)}(t)$. As for minimal cut sets, when the upper bounds of $\hat{W}_{0}^{(1)}(t)$ and $Q_{0}(t)$ are their exact values, then the upper bounds for $W_{0}(t)$ and $\Lambda_{0}(t)$ are also, respectively, their exact values. When the lower bounds of $\hat{W}_{0}^{(1)}(t)$ and $Q_{0}(t)$ are their exact values, then the lower bounds for $W_{0}(t)$ and $\Lambda_{0}(t)$ are also, respectively, their exact values. Tables 17 and 18 , at the end of this section, summarize the above discussions concerning the output group "DIFFERENTIAL CHARACTERISTICS LAST BRACKETS".

The last output group of system characteristics is entitled "INTEGRAL CHARACTERISTICS - BEST BRACKEIS" and contains the best bounds for the integral system characteristics. Table 19 translates the program output to the symbols used in previous discussions. It is noted that these best brackets for the integral characteristics are derived from the best brackets for the differential characteristics (ie, from the output group "DIFFERENTIAL CHARACTERISTICS - BEST BRACKETS"). Therefore, if the upper bounds for the differential characteristics are their exact values, then the upper bounds for the integral characteristics are also their respective exact values. If the lower bounds for the differential characteristics are their exact values, then the lower bounds for the integral characteristics are also their respective exact values:

The output of reliability characteristics for the first parameter run is completed (with ISTOP. = 2). If there are succeeding parameter mus. (NPROB > I), then all this output will be repeated, in the same format. 
TABLE 10.

KEY TO "COMPONENT AND INHIBIT INFORMATION"

\begin{tabular}{|c|l|}
\hline Program Output & \multicolumn{1}{|c|}{ Information } \\
\hline T (hours) & $t$, time (in hours) \\
Q & $q(t)$, the component failed probability \\
W & $w(t)$, the component failure rate (per hour) \\
L & $\lambda$, the (input) component failure intensity \\
& $t$ \\
WSUM & $\int^{t} w\left(t^{\prime}\right) d t^{\prime}$, the expected number of failures \\
& $1-\exp (-\lambda t)$, the probability of one or more \\
FSUM & \\
\hline
\end{tabular}

TABLE 11.

KEY TO "MINIMAL SET INFORMATION" IF MINIMAL CUT SETS ARE USED

\begin{tabular}{|c|c|}
\hline Program Output & \multicolumn{1}{|c|}{ Information } \\
\hline T (hours) & $t$, time (in hours) \\
$Q$ & $\check{Q}(t)$, the minimal cut set failed probability \\
$W$ & $\check{W}(t)$, the minimal cut set failure rate \\
(per hour)
\end{tabular}


TABLE 12.

KEY TO "MINIMAL SET INFORMATION" IF MINIMAL PATH SETS ARE USED

\begin{tabular}{|c|c|}
\hline Program Output & Information \\
\hline$T$ (hours) & $t$, time (in hours) \\
\hline$P$ & 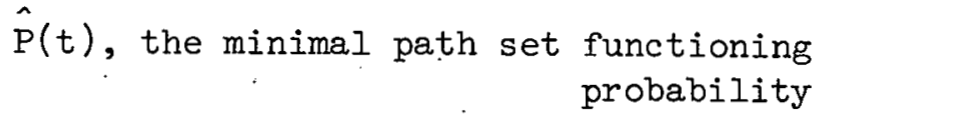 \\
\hline W & $\begin{array}{r}\hat{W}(t) \text {, the minimal path set failure rate } \\
\text { (per hour) }\end{array}$ \\
\hline L & $\begin{array}{r}\hat{\Lambda}(t) \text {, the minimal path set failure intensity } \\
\text { (per hour) }\end{array}$ \\
\hline WSUM & $\begin{array}{r}\int_{0}^{t} \hat{W}\left(t^{\prime}\right) d t^{\prime} \text {, the expected number of failures } \\
\text { to time } t\end{array}$ \\
\hline NO FSUM & 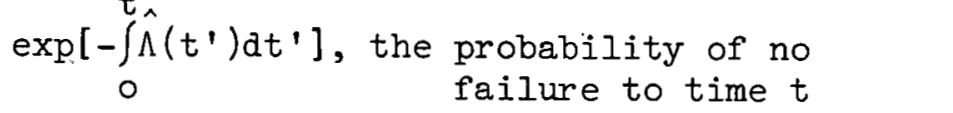 \\
\hline
\end{tabular}


TABLE 13.

KEY TO "SYSTEM INFORMATION - UPPER BOUNDS"

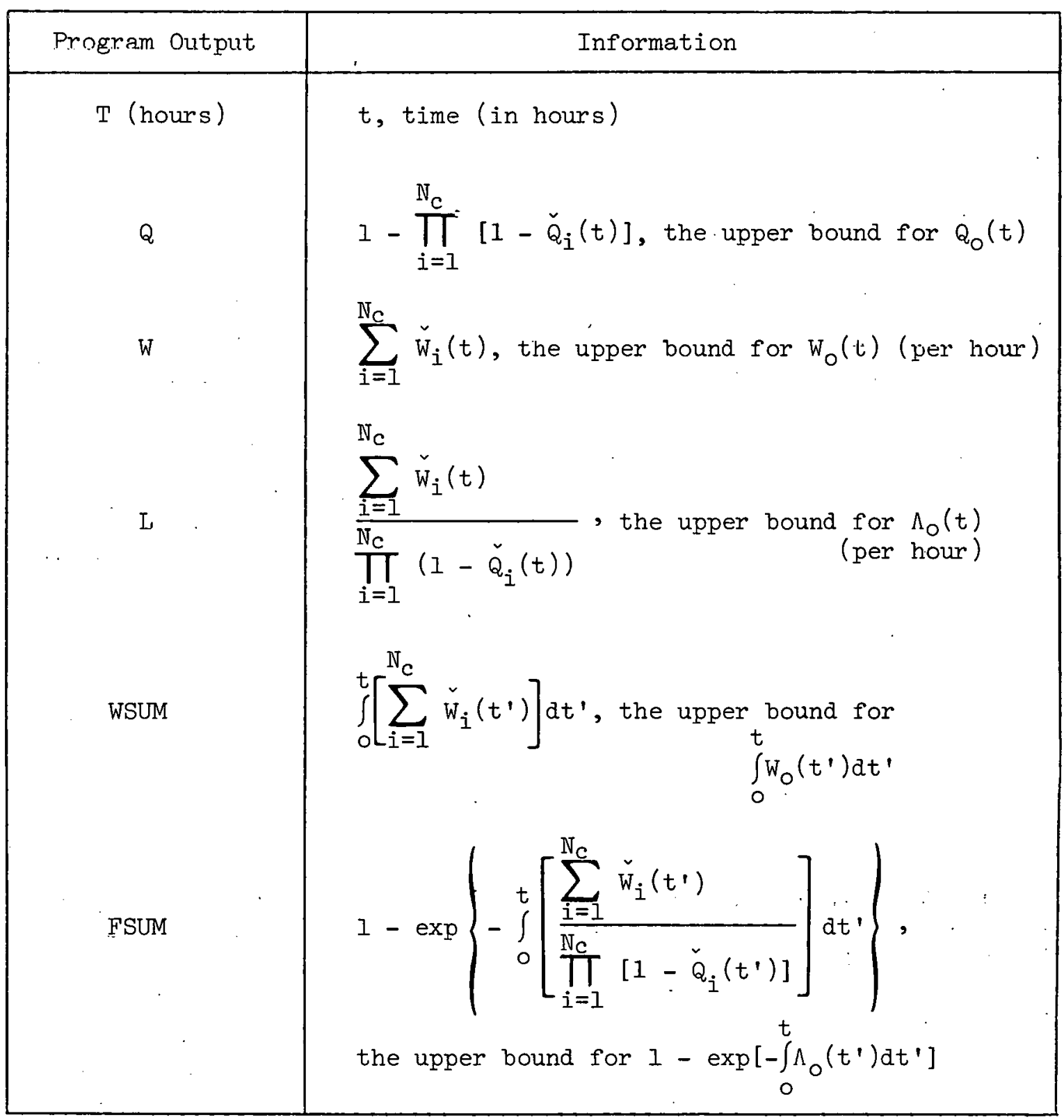


TABLE 14.

KEY TO "DIFFERENTIAL CHARACTERISTICS - BEST BRACKETS"

\begin{tabular}{|c|c|}
\hline Program Output & Information \\
\hline QMIN & $\begin{array}{r}Q_{0}(t)_{\min } \text {, the best lower bound for the } \\
\text { system failed probability } Q_{0}(t)\end{array}$ \\
\hline QMAX & $Q_{0}(t)_{\max }$, the best upper bound for $Q_{0}(t)$ \\
\hline WMTN & $\begin{array}{l}W_{0}(t)_{\min } \text {, the best lower bound for the } \\
\text { system failure rate } W_{0}(t) \text { (per hour) }\end{array}$ \\
\hline WMAX & $\begin{array}{r}W_{0}(t)_{\max }, \text { the best upper bound for } W_{0}(t) \\
\text { (per hour) }\end{array}$ \\
\hline LMIN & $\begin{array}{r}\Lambda_{0}(t)_{\min } \text {, the best lower bound for the } \\
\text { system failure intensity } \Lambda_{0}(t) \\
\text { (per hour) }\end{array}$ \\
\hline IMAX & $\begin{array}{r}\Lambda_{0}(t)_{\max } \text {, the best upper bound for } \Lambda_{0}(t) \\
\text { (per hour) }\end{array}$ \\
\hline
\end{tabular}

TABLE 15.

KEY TO "FAILURE RATE CONTRIBUTIONS" IF MINIMAL CUT SETS ARE USED

\begin{tabular}{|c|c|}
\hline Program Output & Information \\
\hline WIMIN & $\begin{array}{r}\check{w}_{0}^{(1)}(t)_{\min } \text {, the best lower bound for } \stackrel{\mathrm{W}}{O}_{0}^{(1)}(t) \\
\text { (per hour) }\end{array}$ \\
\hline WIMAX & $\begin{array}{r}\check{\mathrm{W}}_{0}^{(1)}(t)_{\max } \text {, the best upper bound for } \tilde{W}_{0}^{(1)}(t) \\
\text { (per hour) }\end{array}$ \\
\hline W'MIN & $\begin{array}{r}\check{\mathrm{W}}_{\mathrm{O}}^{(2)}(t)_{\min } \text {, the best lower bound for } \ddot{W}_{0}^{(2)}(t) \\
\text { (per hour) }\end{array}$ \\
\hline W2MAX & $\begin{array}{r}\check{W}_{0}^{(2)}(t)_{\max }, \text { the best upper bound for } \tilde{W}_{0}^{(2)}(t) \\
\text { (per hour) }\end{array}$ \\
\hline MIN and WMAX & best bounds for $W_{0}(t)$, repeated from Table 14 . \\
\hline
\end{tabular}


TABLE 16.

KEY TO "FAILURE RATE CONTRIBUTIONS" IF MINIMAL PATH SETS ARE USED

\begin{tabular}{|c|c|}
\hline Program Output & Information \\
\hline WIMIN & $\begin{array}{r}\hat{\mathrm{W}}_{\mathrm{O}}^{(1)}(t)_{\min } \text {, the best lower bound for } \hat{\mathrm{W}}_{\mathrm{O}}^{(1)}(t) \\
\text { (per hour) }\end{array}$ \\
\hline WIMAX & $\begin{array}{r}\hat{W}_{0}^{(l)}(t)_{\max } \text {, the best upper bound for } \hat{W}_{0}^{(1)}(t) \\
\text { (per hour) }\end{array}$ \\
\hline W2MIN & $\begin{array}{r}\hat{W}_{0}^{(2)}(t)_{\text {min }} \text {, the best lower bound for } \hat{W}_{O}^{(2)}(t) \\
\text { (per hour) }\end{array}$ \\
\hline W2MAX & $\hat{\mathrm{W}}_{\mathrm{O}}^{(2)}(\mathrm{t})_{\max }$, the best upper bound for $\hat{\mathrm{W}}_{\mathrm{O}}^{(2)}(\mathrm{t})$ \\
\hline (WMIN and WMAX are & e best bounds for $W_{0}(t)$, repeated from Table 14.) \\
\hline
\end{tabular}

TABLE 17.

KEY TO "DIFFERENTIAL CHARACTERISTICS - LAST BRACKETS"

IF MINIMAL CUT SETS ARE USED

\begin{tabular}{|c|c|}
\hline Program Output & Information \\
\hline If the exact values for system characteristics are obtained: \\
\hline QLAST & $Q_{0}(t)$, the exact value for the system failed \\
probability \\
WILAST \\
$\left.\begin{array}{l}\text { W2MIN-LAS'I } \\
\text { W2MAX-IAST }\end{array}\right\}$ & $\begin{array}{l}\tilde{W}_{0}^{(1)}(t), \text { its exact value (per hour) } \\
\mathrm{W}_{0}^{(2)}(t), \text { its exact value (per hour) }\end{array}$ \\
\hline
\end{tabular}


TABLE 18.

KEY TO "DIFFERENTIAL CHARACTERISTICS - LAST BRACKETS"

IF MINIMAL PATH SETS ARE USED

\begin{tabular}{|c|c|}
\hline Program Output & Information \\
\hline If the exact values for system characteristics are obtained: \\
\hline QLAST & $Q_{0}(t)$, the exact value for the system failed \\
probability \\
WILAST \\
$\begin{array}{l}\left\{\begin{array}{l}\text { W2MIN-LAST } \\
\text { W2MAX-LAST }\end{array}\right\} \\
\hat{W}_{0}^{(1)}(t), \text { its exact value (per hour) } \\
\hat{W}_{0}^{(2)}(t), \text { its exact value (per hour) }\end{array}$ \\
\hline
\end{tabular}

TABLE 19.

KEY TO "INTEGRAL CHARACTERISTICS - BEST BRACKETS"

\begin{tabular}{|c|c|}
\hline Program Output & Information \\
\hline $\begin{array}{l}\text { - WMAX-SUM- } \\
\text { FMIN-SUM } \\
\text { FMAX-SUM }\end{array}$ & 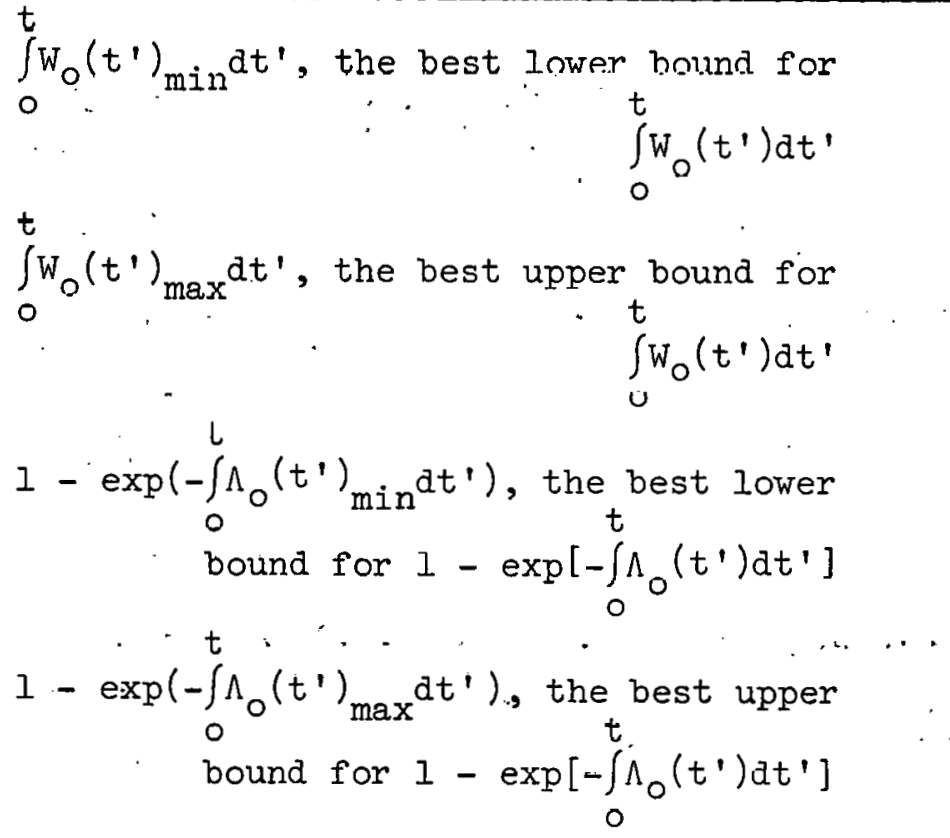 \\
\hline
\end{tabular}




\section{INTRODUCTION}

KITT-2 is simply an extension of KITT-l and is essentially identical to KITT-1 with regard to input and output. Because of this similarity, KITT-2 will be discussed somewhat briefly; many sections will simply reference the pertinent section earlier described for KITT-I.

The difference between KITT-I and KITT-2 is the type of component failure and repair distributions which can be handled. KITT-I can handle components which have constant repair times $(\tau)$ or which are nonrepairable and which have constant failure intensities $(\lambda)$. Moreover, KITT-I is a single phase code; each component must have only one value of $\tau$ and one value of $\lambda$ for all time (or the component must be nonrepairable for all time).

KITT-2 can also handle components which have constant repair times, or which are nonrepairable, and which have constant failure.intensities. However, KITT-2 is a multiphase code. The component during different time intervals, called "phases", may have different reliability properties.

In one time interval, or phase; the component has one value of $\lambda$ and $\tau$. In the next time interval, or phase, the component may have different values for $\lambda$ and $\tau$. Also, in certain phases, the component may be nonrepairable, while in others it may have constant repair times. (The phase description for a component is thus the representation of its time dependence, with regard to its reliability properties, by means of a series of step functions, or histograms.) 
In addition to constant repair times, KTTT-? can also handlc components having constant repair intensities $(\mu)$. A constant repair intensity is equivalent to the component having an exponential repair distribution

$$
b(t)=\exp (-\mu t) \mu
$$

where $b(t)$ is the probability that the component is repaired, per unit time, at time $t$ from the time of failure. For different phases, the component may have different values for $\mu$, or in some phases it may have constant repair intensities $\mu$, while in other phases it may have constant repair times $\tau$ or be nonrepairable.

In KITT-2, if each of the components had one phase, which they can, then KITT-2 would be identical to KITT-1. KITT-2 can therefore handle all the problems which KITT-1 can. KIIT-2, however, is a bit more tedious to input than KITT-1. Therefore, KITT-1 should be run when all the components have only one phase, ie, when "steady state" behavior is to be analyzed. Also, in one computer run, KITT-I can analyze any number of parameter runs, enabling studies to be done on the effect of component data on system reliability. In one computer run, KITT-2 can handle only one parameter run, where the components have only one set of data (varying from phase to phase).

The capability of KITT-2 to handle phases for a component allows the study of the "kinetic behavior" of a system. This allows, for example, the study of the reliability of a system which is under "stress" at certain times (ie, the components have higher $\lambda$ 's at these times). In KITT-2, each component may have up to 50 unique phases, arbitrarily spaced, and hence detailed, complex behavior can be investigated. The multiphase capability of KITT-2 also allows the mock-up, by a series of histograms, of any type of time-dependent failure intensity $\lambda(t)$ and any type of repair distribution. 
The next section gives illustrations of this approximating by histograms, along with an illustration of a general multiphase component.

KITT-2 requires as input the phase durations for each component and the values of $\lambda, \tau$, etc, in each phase. Like KITT-1, KITT-2 also requires the unique minimal cut sets or unique minimal path sets of the fault tree. KITT-2 obtains exactly the same reliability information as KITT-1 does. Exact, time-dependent reliability characteristics are obtained for each component and minimal cut or path set. For the system, upper bound approximations are obtained for the characteristics, or the characteristics are enveloped by the bracketing procedure. If the bracketing procedure is carried to completion, then the exact values for the system characteristics are obtained.

\section{MULTIPHASE ILLUSTRATIONS}

\section{The Component with a Number of Phases}

In this section illustrations are given of multiphase descriptions for a component. The illustrations will help to clarify the describing of the time-dependent behavior of a component by means of "phases". The illustrations will also be used to introduce the terminology which will be used in later discussions.

Figure 6 on the next page is an illustration of a component having three phases with regard to its failure intensity $(\lambda)$. In Figure 6 , the horizontal axis $t$ depicts time while the vertical axis $\lambda$ depicts the value of the component failure intensity. During Phase 1 , which lasts from $t=$ 0 to $t=t_{l}$, the component has a constant failure intensity $\lambda_{1}$. During Phase 2 , the component has . different failure intensity $\lambda_{2}$. Phase 2 lasts from $t=t_{1}$ to $t=t_{2}$. Final. $y$, in Phase 3 which begins at $t=t_{2}$, the component failure intensity has still another constant value $\lambda_{3}$. 


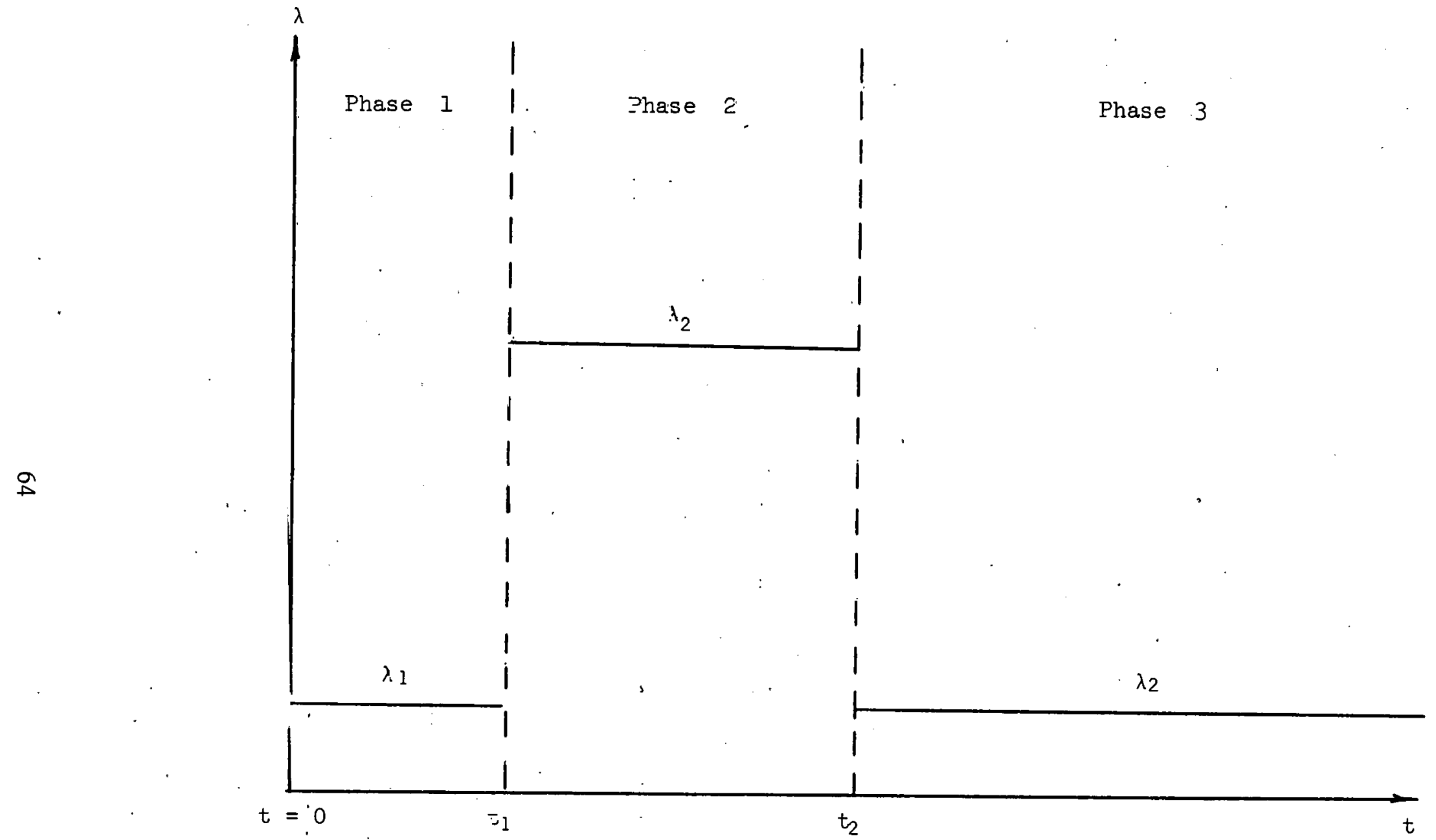

FInURE 6. A Three-Phase Component 
Figure 6 can represent, for example, the component passing from a standby status to an operating status and then back to a standby status. In Phase 1, the component is on standby and therefore has a low value of $\lambda, \lambda_{1}$. The component is called upon to operate at $t=t_{1}$ and the duration of operation lasts until $t=t_{2}$. During this time period, Phase 2 , the component is under "stress" and the value of $\lambda$ is much higher, $\lambda_{2}$. At time $t=t_{2}$, the component is again put on a standby basis, thereby advancing into Phase 3.

The more general multiphase description of a component is illustrated in Figure 7. The component has a total of $N$ phases. During the general $n^{\text {th }}$ phase, the component has a value of $\lambda_{n}$ for its failure intensity and has a value of $\tau_{n}$ for its constant repair time. As will be elaborated upon later, a negative sign attached to $\lambda_{n}$ or ${ }^{\tau} n$ will denote the component is nonrepairable during Phase $\mathrm{n}$ or that the component has a constant repair intensity $\mu_{\mathrm{n}}$ in this phase.

The general Phase $n$ will be said to have its left boundary at $t_{\hat{n}-1}$, and its right boundary at $t_{n}$. Phase $n$ will also be said to have a beginning time of $t_{n-1}$ and an end time of $t_{n}$. The left boundary therefore corresponds to the beginning time and the right boundary to the end time. This naming procedure will aid later in a more compact description of the output quantities yielded by KITT-2.

\section{The Mock-Up of a General Time-Dependent Failure Intensity}

Figure 8 depicts how phases may be used to approximate any continuous time-dependent failure intensity $\lambda(t)$. In this example, the component has an actual failure intensity represented by the continuous curve. The component is made to have phases in order to approximate the true $\lambda(t)$. The time scale is divided into intervals, or phases ( 4 phases for the example), 


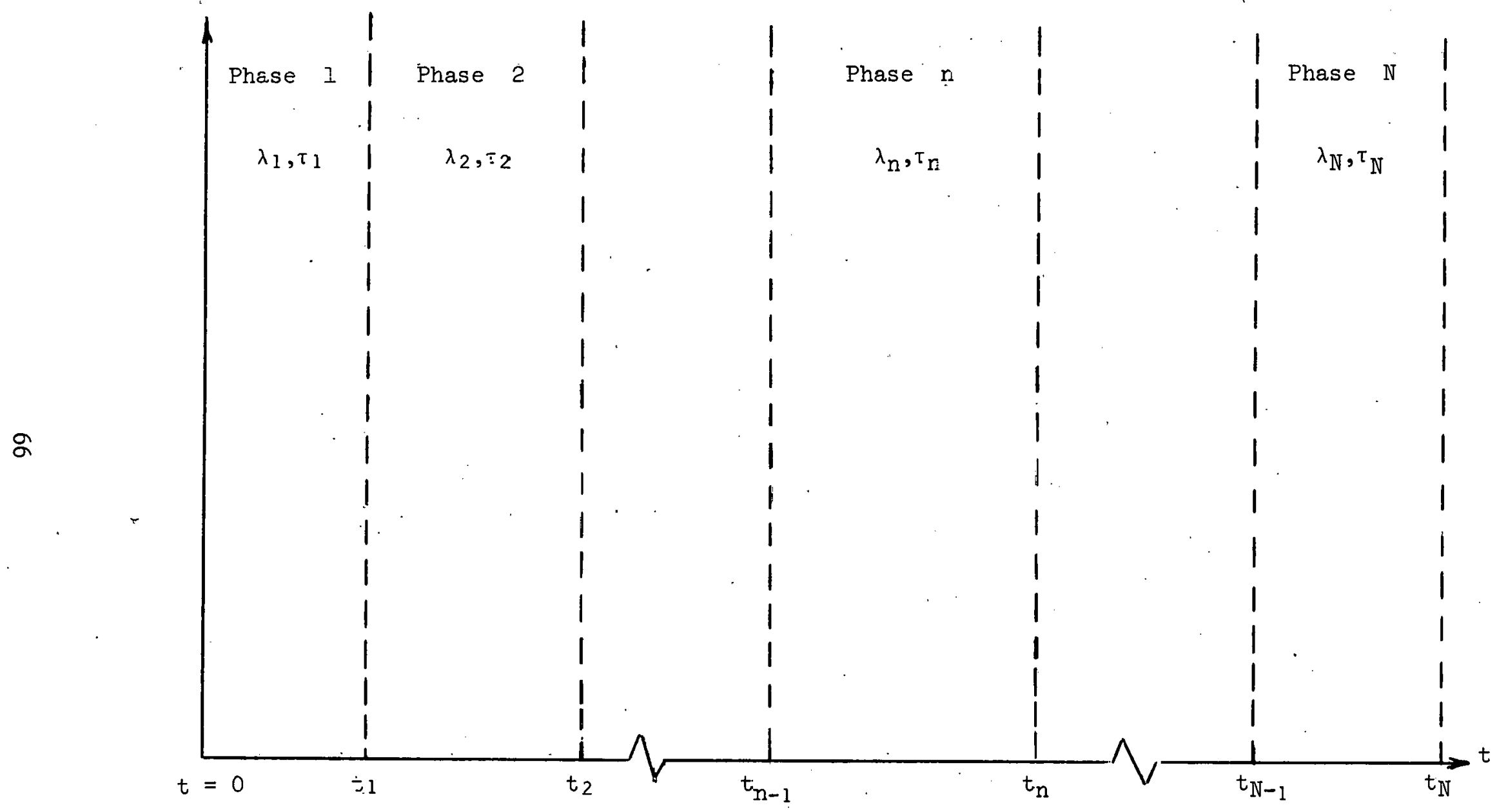

FIGURE 7. The General N-Phase Component 


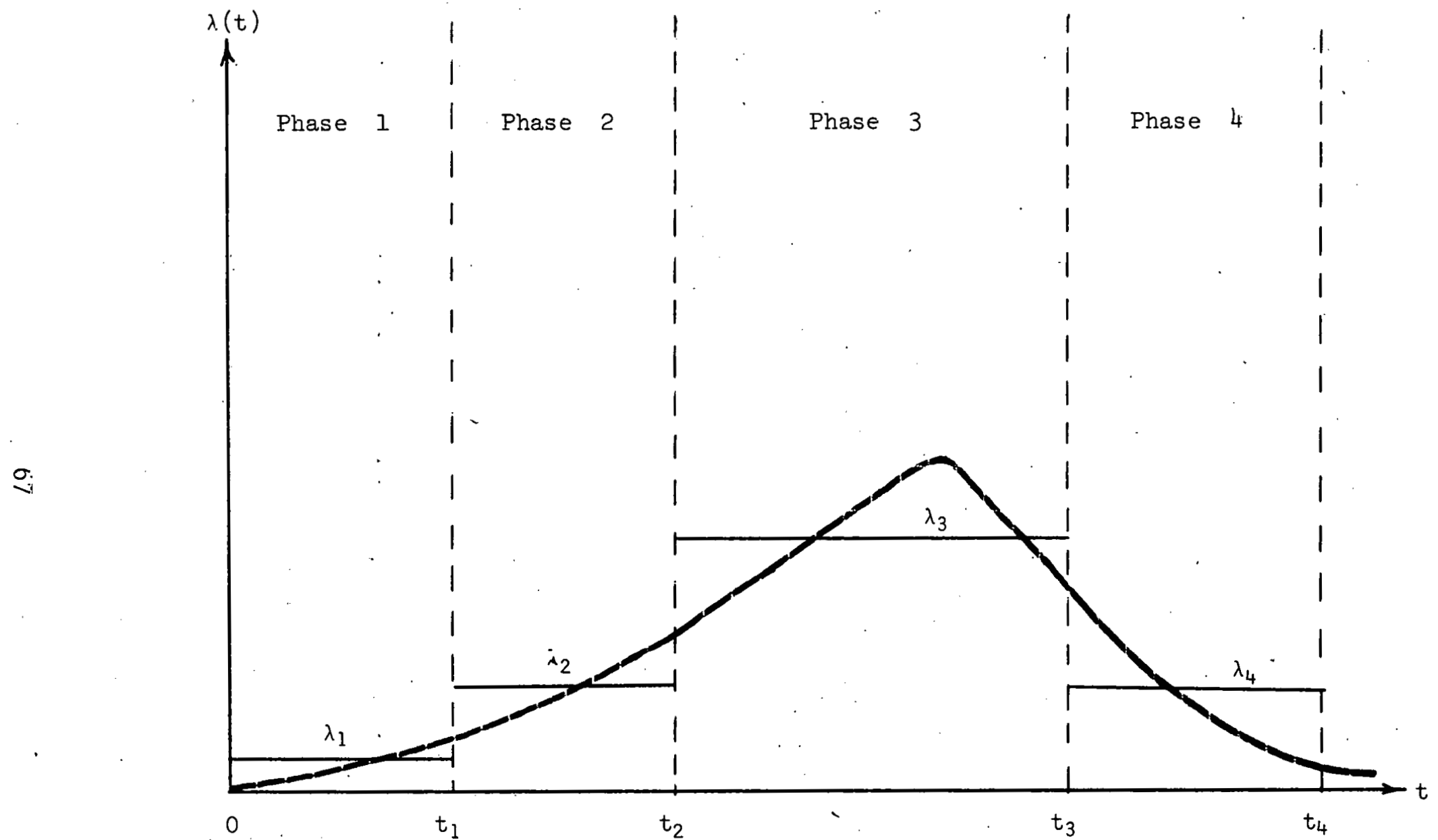

FIGURE 8. Mock-Up of a Time-Dependent Failure Intensity 
so that the curve may be approximated by a series of histograms, where the histograms represent constant values of $\lambda$ in the particular phases. The phases are chosen to best fit the actual curve. The end time of the last phase, $t_{4}$ in the figure, corresponds to a maximum time of interest, beyond which time no reliability information is desired. The repair data for the component can be easily factored into this phase representation. If, for example, the component had one repair time $\tau$ which was the same for all time $t$, then each phase in Figure 8 would, in addition, be assigned this value of $\tau$. The use of phases, then, allows one to approximate any type of failure intensity $\lambda(t)$. Since KITT-2 allows up to 50 phases for a component, arbitrarily spaced, the approximation may be made as close as desired.

\section{The Mock-Up of a General Repair Distribution}

Any repair distribution, whether it. be normal or Weibull or any other, can be represented by a corresponding time-dependent repair intensity $\mu(t)^{[a]}$. Therefore, approximating the repair distribution is equivalent to approximating its corresponding repair intensity $\mu(t)$. Phases can be used to approximate $\mu(t)$ in the same way as they were used in the previous section to approximate a general failure intensity $\lambda(t)$.

Figure 9 illustrates this approximating of a general repair intensity by the method of phases. As previous 1.y stated, KITT-2 can handle constant repair intensities for one or more of the phases, in addition to constant repair times or nonrepairability. The constant repair intensities are used in Figure 9 to approximate an actual repair intensity (the solid curve)

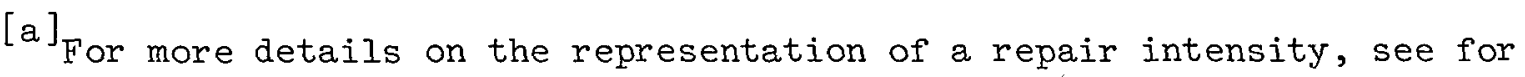
example, A. M. Polovko, Fundamentals of Reliability Theory; New York; Academic Press, 1968. 


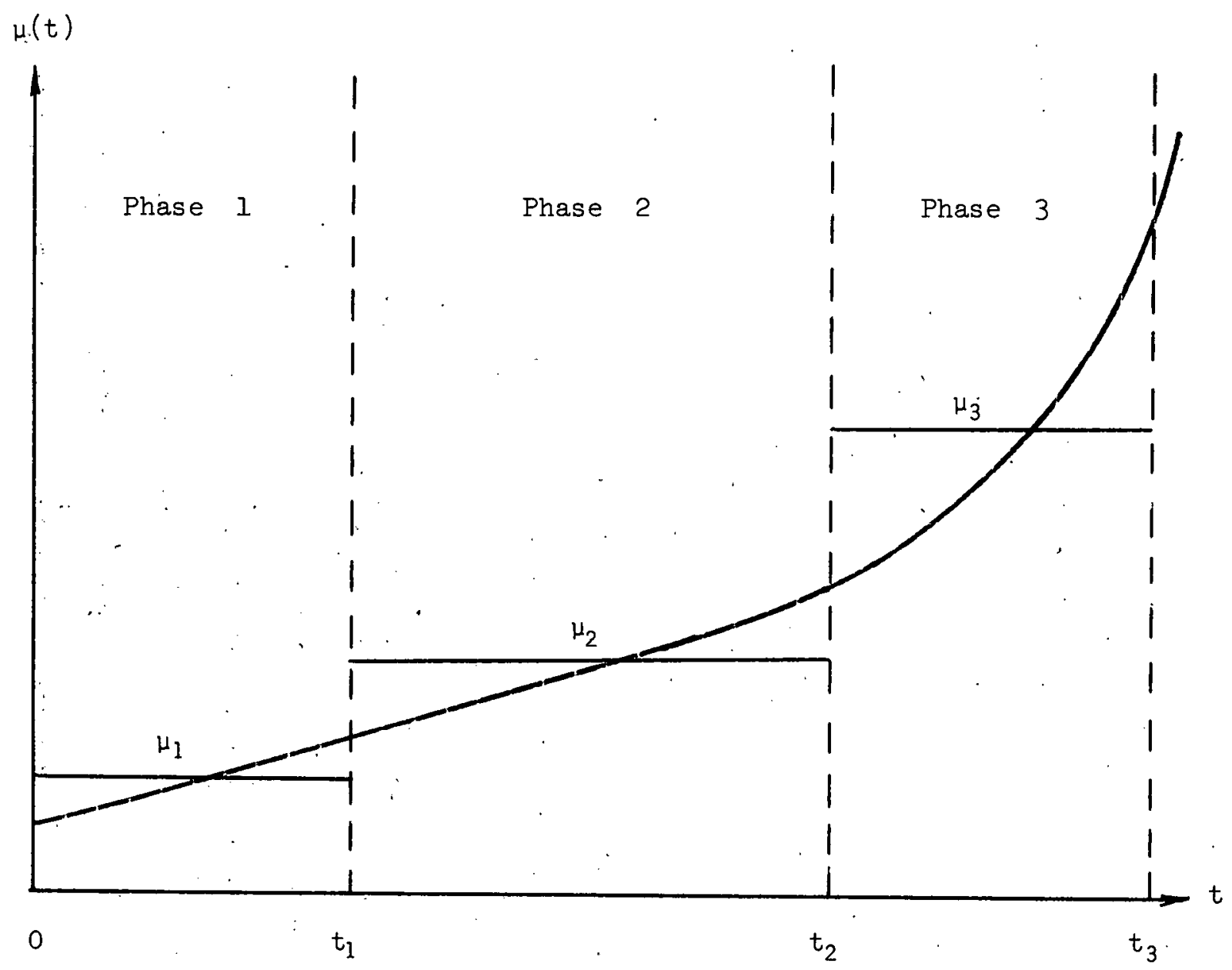

FIGURE 9. Mock-Up of a Time-Dependent Repair Intensity 
by a series of histograms. The component whose actual repair intensity is given by the curve is (arbitrarily) made to have 3 phases. The constant repair intensities $\mu_{1}, \mu_{2}$, and $\mu_{3}$ in Figure 9 are those for Phases 1,2 , and 3 , respectively, and these phases and repair intensities are chosen to best fit the actual repair intensity. Using more phases (i.e, more histograms) will give a better fit to the curve. As in the approximating of $\lambda(t)$ in the previous section, one can therefore approximate $\mu(t)$ as closely as desired since up to 50 phases per component are allowed in KITT-2.

A component may have only one set of phases. Therefore, if phases are used to approximate both $\lambda(t)$ (Figure 8) and $\mu(t)$ (Figure 9), then one set of phases shorld be chosen so that both $\lambda(t)$ and $\mu(t)$ are well approximated. This may involve choosing a set of phases for $\lambda(t)$ and a set of phases for $\mu(t)$ and then using the smaller set. A number of smaller consecutive phases with the same value of $\lambda$ or $\mu$ will give exactly the same results as one phase having this value of $\lambda$ or $\mu$ which incorporates these smalier phases.

\section{The Inhibit Condition with a Number of Phases}

The last example of the use of phases is with regard to an inhibit condition. An inhibit condition in a fault tree is a necessary event which must exist before failures can be propagated up the tree. An "occurrence probability" is therefore associated with each inhibit condition, which is the probability of that event existing. Phases may be used for an inhibit condition, therefore, when the probability of occurrence for that event, changes from time to time. Figure 10 illustrates this, where the horizontal bars denote different probabilities of occurrence for a particular inhibit condition for different periods of time. 


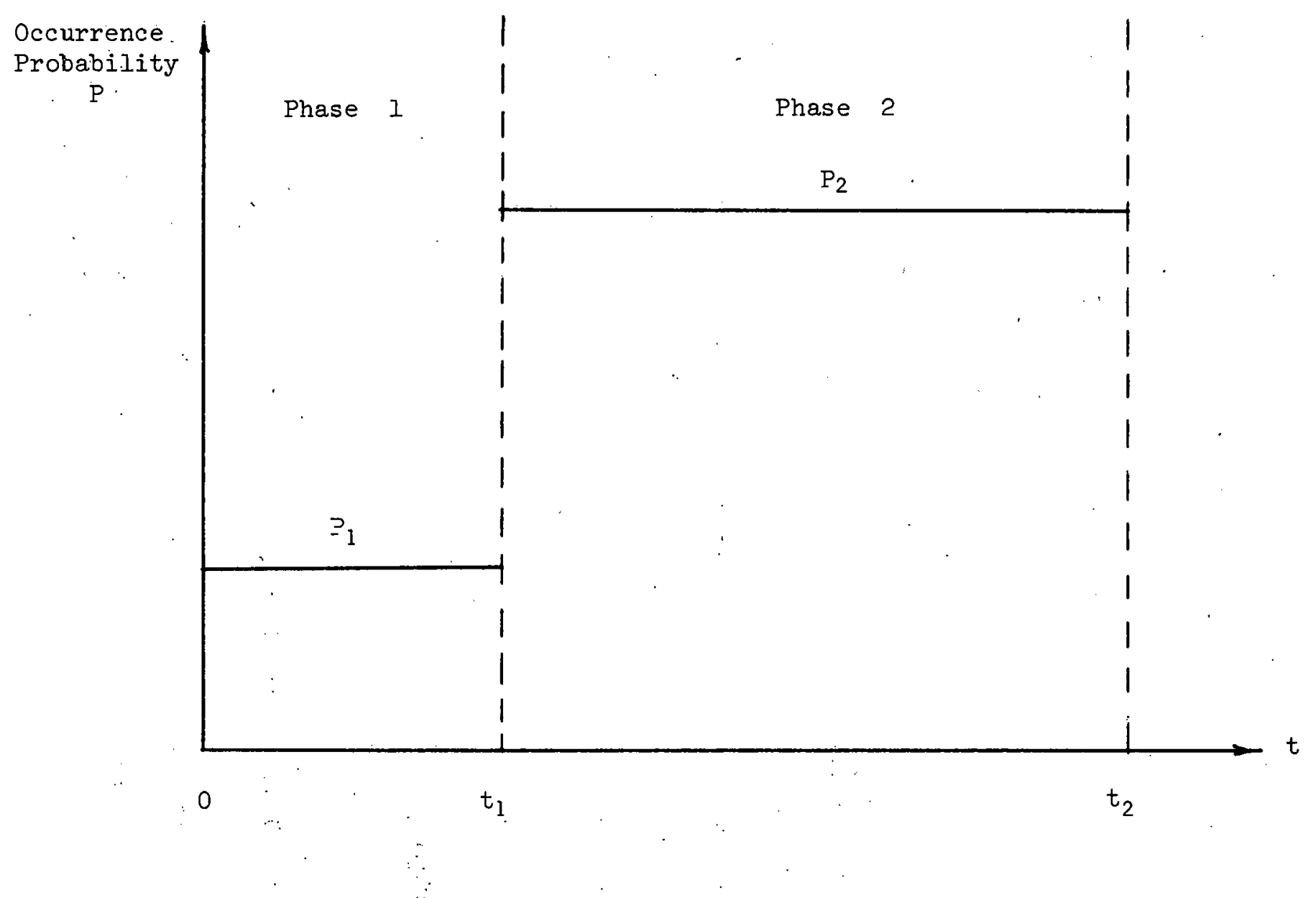

FIGURE 10. The Murtiphase Inhibit Condition 


\section{RELIABILITY INFORMATION OBTAINED BY KITT-2}

The reliability characteristics yielded by KITT-2 are exactly the same as those yielded by KITT-1. The characteristics will of course have different values because of the different problems being analyzed; however the same characteristics are obtained. The user is therefore referred to the section "RELIABILITY INFORMATION OBTAINED BY KITT-l" on page 2, which contains the detailed description of the reliability quantities also obtained by KITT-2.

\section{INPUT DESCRIPTION}

This section describes the data which must be input to KITT-2. The general input formats, either an F10.0 or an I10, are the same as those used for KITT-1. A large portion of the input to KITT-2 is identical to that for KI'l'I-I, and for such input the user is merely referred to the pertinent input group of KITT-1.

\section{Input Group 1}

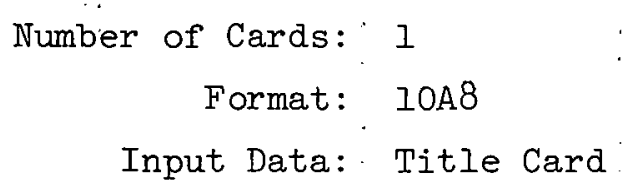

This card is used for description of the problem to be run. Any alphanumeric characters may be used in the 80 columns of the card. 


\section{Input Group 2}

Number of Cards: I

Format: $\quad$ II0

Input Data: NCOMP ( $1 \leq \operatorname{NCOMP} \leq 350)$

NCOMP is the total number of unique components plus inhibit conditions. This total number must include the number of unique components and inhibit conditions which are in one or more of the minimal cut sets or minimal path sets. If additional components are input which are not in a minimal cut set or path set, then reliability characteristics are printed out for the components, and these components are ignored for system computations. (NCOMP, therefore, exactly corresponds to the NCOMP of Input Group 3 for KITT-1.)

\section{Input Group 3}

Number of Cards: 1

Format: $2 I I 0$, FIO.O

Input Data: NTPT, NOUT,DELTA $2<$ NTPT $\leq 50$

NOUT $\geq 1$

NTPT is the total number of time points for which characteristics are obtained for components, sets, and the system; the total number of time points used does not affect the computer time. NOUT is the printout multiple for component and set characteristics; if NOUT equals one, these characteristics are printed out.for every time point; if it equals two, they are printed out for every second time point, and so forth. 'DELTA is the spacing between the time points. If DELTA is read in as a positive value $\Delta t$, then it is taken to be the spacing in hours between the time points; characteristics are then obtained for times of $0, \Delta t, 2 \Delta t, \ldots \ldots$ 
(NTPT-I) $\Delta$ t. If DELTA is read in as 0.0 , or any negative number, then the NTPT time points, arbitrarily spaced, are explicitly read in (Input Group 4 ). The variables NTPT, NOUT, and DELTA are exactly the same as those of Input Group 7 for KITT-I. As stated there, the accuracy of the differential characteristics is not in any way influenced by the time points chosen. The accuracy of the integral characteristics, obtained from a trapezoidal integration, is however affected by the time points. Therefore, if accurate integral characteristics are desired it is preferrable to explicitly read in the time points (reading in 0.0 or a negative number for DELTA). It equal spacing between the time points is used, then NTPT should be set equal to 50 and DELTA should be determined from Equation (9) in Input Group 7 for KITT-1.

\title{
Input Group 4
}

\author{
Number of Cards: Varies \\ Format: $8 \mathrm{~F} 10.0$ \\ Input Data: TOT $(J) ; J=1, N T P T$
}

This Input Group is skipped if DELTA is positive (Input Group 3 ).

TOT $(J)$ are the time points at which characteristics are obtained for components, sets, and the system, if DELTA is read in as 0.0 or a negative number. The time points may be arbitrarily spaced and may begin at any nonnegative time $[\operatorname{TOT}(1) \geq 0.0]$. They must, however, be input in increasing $\operatorname{order}[\operatorname{TOT}(1)<\operatorname{TOT}(2)<\ldots<\operatorname{TOT}(\mathrm{NTPT})]$. This input group is the same as Input Group 8 for. KITT-1. As stated there, the integral characteristics. are always obtained with the integrals starting at TOT(1). Explicitly 
reading in the time points allows one to obtain maximum detail and accuracy for a problem. As previously discussed, the accuracy of the differential characteristics is independent of the time points chosen. The integral characteristics are obtained using a trapezoidal integration, and, consequently, their accuracy is affected by the time points chosen. For accurate integral characteristics, then, the time points should be chosen so that the time points are more closely spaced in areas where the differential characteristics are more rapidly changing in value.

For KITT-2, the placing of time points for accurate integral characteristics involves, as in KITT-1, placing several points near TOT(1), the origin. [For example, using several points after TOT(1), which are spaced $\tau_{a}$ apart, where $\tau_{a}$ is an average component repair time.] It also involves placing time points near the boundaries of component phases which differ radically from their neighboring phases.

The very use of component phases causes discontinuities in $\lambda$ across phase boundaries. This in turn causes discontinuities in the component failure rate $w(t)$ across the phase boundaries. In fact, the step difference in $w(t)$ from one phase to another is approximately equal to the step difference in $\lambda$ across the phase. Since $w(t)$ and $\lambda$ are the differential characteristics which are integrated to obtain the integral characteristics, both for the components themselves and for the minimal cut or path sets and the system, placing time points on either side of phase boundaries accounts for these discontinuities when the integrals are computed. Because of the increased accuracy gained by integrating any quantity, this practically involves placing a time point near the boundaries (say one hour after the beginning time and one hour before the end time) of only those phases which are radically different from their neighboring phases, eg, those phases which have an order of magnitude change in $\lambda$. After experience is 
gained of the general behavior of these characteristics (gained chiefly from running the code) the user will have little trouble in the placing of these time points ${ }^{[a]}$. Another approach in the placing of time points is to make one run using equal spaced time points in order to obtain a general behavior of the differential characteristics which are of interest and which are integrated for the integral characteristics. From this first run, the areas can be discerned where the differential characteristics are rapidly changing. (have large derivatives). A more refined set of time points can then be chosen such that these areas have time points more closely spaced. Using this refined set of time points, a second computer run can then be made to obtain the accurate reliability characteristics desired. This approach, though a bit tedious, is not impractical since the computer times needed for each run are generally quite small, on the order of fractions of a minute.

\section{Input Group 5}

Number of Cards: 1

Format: IIO

Input Data: ISTOP

ISTOP is the bracket flag, having a value of 1 or 2 . If ISTOP $=2$, syslem reliability characteristics are obtained by bracketing. -If ISTOP $=1$, they are not.

[a] As will be explained later (Input Group 14), several computer runs can also be made, where in each computer run characteristics are obtained only up to a certain time and are then continued in the next run. Two such runs would, for example, allow 100 points to be used for a problem. 
ISTOP for this input group is exactly the same as that for Input Group 6 for KITT-I and is used in exactly the same way. The reader is therefore referred to Input Group 6 for KITT-I; every part of that discussion is valid here.

\section{Input Group 6}

Number of Cards: I

Format: $2 I 10$

Input Data: NBMAX, IFAG2 $1 \leq \mathrm{NBMAX} \leq 100$

This Input Group is skipped if ISTOP

$=1$ (Input Group 5).

NBMAX is the number of outer brackets to obtain. IFAG2 is the system failure rate correction flag and has a value of either 1 or 2 . For IFAG2 $=2$, the correction term, $\check{W}_{0}^{(2)}(t)$ or $\hat{W}_{0}^{(2)}(t)$, is bracketed; for IFAG2 = 1 , this term is not computed.

The variables NBMAX and IFAG2 are exactly the same as those of Input Group 9 for KITT-I and they are used in exactly the same way. The reader is consequently referred to this input group of KITT-1 for the discussions on NBMAX and IFAG2. All parts of those discussions are valid here.

\section{Input Group 7}

Number of Cards: Varies

Format: $8 I 10$

Input Data: $N B 2(N) ; N=1, \operatorname{NBMAX} 1 \leq \mathrm{NB} 2(\mathrm{~N}) \leq 30$ 


$$
\begin{aligned}
& \text { This Input Group is skipped if IFAG2 } \\
& =1 \text { (Input Group 6) or if ISTOP = I } \\
& \text { (Input Group 5). }
\end{aligned}
$$

NB2(N) is the number of inner brackets to use for each outer bracket $N$, if the correction term for $W_{0}(t)$ is computed.

The input variable $\mathrm{NB2}(\mathbb{N})$ is identical to that for Input Group 10 of KITT-1. The reader is referred to that discussion for a description of the use of NB2(N). Again, every part of that discussion is valid here. The Recommendations for Input Groups 5, 6, and 7 of KITT-2 are exactly the same as the recommendations given for the corresponding input groups of KITT-1, Input Groups 6, 9, and 10. These recommendations are given beginning on page 30 , with the summary of these recommendations being given on page 41 .

For Each Component Read In 'I'he Following Seven Input Groups (Input Groups

8 Through 14). These Input Groups Are Input In Order For The Component. Repeat These Seven Input Groups For As Many Components As There Are. These Input Groups Apply Only To Components; Inlipit Conditions Are Read In Elsewhere.

Input Group 8 (First Group of the Component Group Set) Number of Cards: 1

Format: $2 I 10$

Input Data: IDEX, NPHASE $1 \leq$ NPHASE $\leq 50$

IDEX is the component index, and NPHASE is the number of phases for the component. 
The components and inhibit conditions are assigned arbitrary indices for identification. For the NCOMP total components and inhibit conditions (Input Group 2), however, the indices assigned must run consecutively from 1 to NCOMP. (If the PREP Code is used to obtain the minimal cut sets or path sets, then these indices are automatically assigned by it.) The order in which the components are read in is completely arbitrary; the component group sets, where a group set is Input Groups 8 through 14 needed for each component; can be input in any order with respect to the indices assigned. IDEX, therefore, is the index assigned to the component and identifies the group set (Input Groups 8 through 14) as being for the component. NPHASE is the tota. number of phases for this component; in Figurc 7 on page 66, NPHASE would correspond to $\mathbb{N}$. The component must have at least $I$ phase and not more than 50 phases.

For added clarity, we shall illustrate the indexing of components and inhibit conditions by a simple example. Consider the situation where there are two components and one inhibit condition. The user, for example, assigns one component an index of 1 , the inhibit condition an index of 2 ; and the other component an index of 3. Any other assignment of the three indices 1,2 , and 3 is equally valid. When the data are read in for the inhibit condition (Input Groups 15 through 17), a minus sign will be attached to its index to denote it as an inhibit condition; -2 would be read in for our example. The component data read in would have the actual component indices, $i$ and 3, as part of their input. The group "set (Input Groups 8 through 14) would be read in for one of the components and then would be immediately repeated for the other component. The group sets could be input in any order; for example the group set for the component of index 3 (having IDEX = 3) could be read in before that for the component of index I (having IDEX = I). The data for the inhibit condition would be read in 
later (Input Groups 15 through 17), in fact immediately after the two input. group sets are read in for the components.

\section{Input Group 9 (Second Group of the Component Group Set)}

Number of Cards: Varies

Format: $8 \mathrm{~F} 10.0$

Input Data: $\operatorname{TP}(\mathrm{L}) ; \mathrm{L}=1, \mathrm{NPHASE}$

TP(L) are the phase end times (in hours) for the component phases (NPHASE of them). The phase end times must be input in order of phase number; ie, $T P(1)$ is the end time for Phase 1, TP(2) for Phase 2, etc. Phase $I$ is always assumed to have a beginning time of $t=0.0$

In Figure 7 on page $66, \operatorname{TP}(1)$ corresponds to $t_{1}, \operatorname{TP}(2)$ to $t_{2}, \ldots$ TP(NPHASE) to $t_{N}$, where $N$ corresponds to NPHASE. As in Figure 7 , the beginning time of Phase $I$ is always assumed to be 0.0 . The phases are always assumed to be consecutive; the end time of the $n^{\text {th }}$ phase $t_{n}$ is always assumed to be the beginning time of the $(n+1)^{\text {th }}$ phase.

The end time of the final phase, 'L'P(NPHASE), must always be larger than the maximum time for which reliability characteristics are obtained. Therefore, one must always choose values such that

$$
\operatorname{TP}(\text { NPHASE })>t_{\max }
$$

where

$$
t_{\max }=(N T P T-I) D E L T A
$$

if DELTA is positive (Input Group 3), or

$$
t_{\max }=T O T(N T P T)
$$


if the time points are read in (Input Group 4). The above inequality, Equation (102), must be true for all the components read in.

How much larger TP(NPHASE) is compared to $t_{\max }$ is arbitrary, although it must be large enough to be "seen" by the computer code. Therefore, the only requirement necessary is that

$$
\mathrm{TP}(\mathrm{NPHASE})-t_{\max }>10^{-5} t_{\max }
$$

where the factor $10^{-5}$ is somewhat arbitrary, but is a safe number to use.

\section{Input Group 10 (Third Group of the Component Group Set)} Number of Cards: 1

Format: I10

Input Data: IBPHA

IBPHA is the boundary condition index. If IBPHA is set equal to zero, or is set equal to a negative number, then there are no boundary conditions imposed on the phase boundaries. If IBPHA is read in as a positive integer, then IBPHA is the number of phase boundaries which have imposed boundary conditions; this number does not include the initial boundary condition at $t=0.0$ (ie, the initial condition for the beginning time of the first phase).

A boundary condition is imposed only if certain knowledge is known, or if some event is given. As an example of a boundary condition, we shall consider the initial boundary condition for $t=0$. This initial condition $\ldots \ldots \ldots$ is not one of the boundary conditions read in here, but it will help to illustrate the meaning of imposed boundary conditions. At $t=0$, which is the beginning time of operation (and the beginning time of all the first phases for the components) an initial condition $q(0)$ must be supplied, where $\mathrm{q}(0)$ is the probability that the component is in its failed state 
at the start of operation, $t=0$. Generally, the component is known to be operating at $t=0$, and hence the initial boundary condition which must be supplied is

$$
\begin{aligned}
& q(0)=0 \text {, component known to be } \\
& \text { operating at } t=0 \text {. }
\end{aligned}
$$

If the component is known to be in a failed state at $t=0$, for example being in a state of repair, then the boundary condition supplied is $q(0)$ $=1.00$ since the component is known to be in a failed state. The initial state of the component at $t=0$, whether the component is in its failed state or functioning state, must be known in order to predict (compute) reliability; more generally the probability of the component being in its failed state at $t=0, q(0)$, must be known.

The use of IBPHA to impose boundary conditions at the beginning times of phases other than the first is similar to the use of the initial boundary condition. (The first phase is not included since the boundary condition at the first phase beginning time is the initial boundary condition at $t=0$, input later.) If IBPHA is positive, boundary conditions are imposed at the beginning times of certain of the phases after the first phase, and IBPHA gives the number of such boundary conditions. The boundary conditions are imposed by giving values for $q(t)$ at the phase beginning times. In Figure 7 on page 66, a boundary condition would be imposed on Phase 2 by giving $q\left(t_{1}\right)$. If IBPHA is positive, then the failed probabilities imposed at the phase beginning times are input in the next input groups and IBPHA input here merely gives the total number of such imposed failed probabilities. As stated, if IBPHA is set equal to zero or a negative integer, then there are no imposed boundary conditions (other than the initial condition at $t=0)$. This is generally the case since no failure information is generally known for the component. If the component is repaired when it 
fails, then IBPHA would still be set equal to zero or a negative integer; IBPHA refers only to knowledge of the component state at the phase boundaries. The usual case when IBPHA is not set equal to zero, or a negative integer, is when the component is examined at the end of a phase. For example, assume the component is examined at the end of the $n^{\text {th }}$ phase and if it is in a failed state it is immediately brought back to operation. A boundary condition would then be imposed for the beginning time of the $(n+1)$ th phase

$$
q\left(t_{n}\right)=0
$$

since the component is known to be operating, or to be in its functioning state, at the beginning of the $(n+1)^{\text {th }}$ phase. IBPHA for this situation would then be set equal to 1 . If the component is examined at the end of each of $\mathrm{k}$ phases then IBPHA would be set equal to $\mathrm{k}$ and the boundary conditions $\mathrm{q}=0$ (input in the next groups) would be imposed for the respective phases which immediately follow each of these $k$ phases.

Input Group 11 (Fourth Group of the Component Group Set)

Number of Cards: Varies

Format: 8110

Input Data: $\operatorname{IBPOS}(\mathrm{K}) ; \mathrm{K}=1, \mathrm{IBPHA}$

This Input Group is skipped if IBPHA $\leq 0$ (Input Group 10).

IBPOS $(K)$ are the phase numbers which have imposed boundary conditions at. their bcginning times. As discussed earlier, these imposed boundary conditions do not include the initial condition at $t=0.0$. The phase 
numbers may be input in any order. (For the discussion on imposed boundary conditions, see the previous input group.)

\section{Input Group 12 (Fifth Group of the Component Group Set)}

Number of Cards: Varies

Format: $8 \mathrm{~F} 10.0$

Input Data: $\mathrm{QBPOS}(\mathrm{K}) ; \mathrm{K}=1$, IBPHA

$\approx$

This Input Group is skipped if IBPHA

$\leq 0$ (Input Group io).

$\mathrm{QBPOS}(\mathrm{K})$ are the failed probabilities imposed as boundary conditions. QBPOS $(K)$ is the failed probability imposed at the beginning time of phase $\operatorname{IBPOS}(K)$. QBPOS $(K)$ must therefore correspond to the IBPOS(K) of Tnput Group 11. If $\mathrm{QBPOS}(\mathrm{K})$ is assigned a value greater than 1.00 , then the component will remain failed for the entire duration of the phase IBPOS(K). As discussed in Input Group 10, if the component is examined at the end of certain phases and immediately repaired if failed, then $Q B P O S(K)$ is set equal to 0.0 for those phases immediately after the examinations.

Input Group 13 (Sixth Group of the Component Group Set)

Number of Cards: Varies

Format: 8 Fio.0

Input Data: $\operatorname{XLMDA}\left(I_{1}\right), \operatorname{TAT}(L) ; L=1$, NPHASE

XLMDA(L) and TAU(L) are the component failure intensity $\lambda_{I}$ (per hour) and component repair : time $\tau_{L}$ (hours) for. Phase $I_{1}, T_{1}=1, \ldots$, NPHASE. 
(see Figure 7 on page 66.) The failure intensity and repair time are input pairwise, with four pairs to a card. The failure intensities and repair times must be input in order of phase number; ie, with reference to Figure $7, \lambda_{1}, \tau_{1}, \lambda_{2}, \tau_{2}, \ldots, \lambda_{N}, \tau_{N}$ is the order of input. A negative $\lambda_{L}$ denotes the component is nonrepairable during Phase $L$, the absolute value of $\lambda_{L}$ is then used as the component failure intensity. A negative ${ }^{\tau} \mathrm{L}$ denotes the component has an exponential repair distribution in Phase L [Equation (10I)], the absolute value of $1 / \tau_{L}$ is then used as the constant repair intensity $\mu_{L}$ (per hour) for this phase $\left(\mu_{L}=-1 / \tau_{L}\right)$.

The phase concepts and the assigning of failure intensities, repair times, and repair intensities to phases have been previously discussed. Therefore, no elaboration is necessary here. The list below summarizes the way nonrepairability, etc, is donoted for a phase.

Variables Input $\lambda_{L}>0, \tau_{L}>0$

$$
\lambda_{\mathrm{L}}<0, \tau_{\mathrm{L}}
$$

$$
\therefore \lambda_{L}>0, \because \tau_{L}<0
$$

\section{Meaning}

The component has a constant repair time in Phase $\mathrm{L}$. The value of $\lambda_{L}$ is then the value of the failure intensity (per hour) and $\tau_{I}$ is the value of the constant repair time (hours) for this phase.

The component is nonrepairable in Phase $\mathrm{L}$. The absolute value of $\lambda_{L}\left(-\lambda_{L}\right)$ is then the value of the failure intensity (per hour) for this phase. The value $\tau_{L}$ is ignored and can be input as any value (or left blank).

The component has a constant repair intensity in Phase $\mathrm{L}$. The value of $\lambda_{L}$ is the value of the failure intensity (per hour) and the absolute value of $l / \tau_{L}$ is the repair intensity $\mu_{\mathrm{L}}$ (per hour) for this phase. $\left(\mu_{\mathrm{T}_{1}}=-1 / \tau_{\mathrm{L}}\right)$ 
Input Group 14 (Seventh and Last Group of the Component Group Set)

Number of Cards: I

Format: F10.0

Input Data: Q,0O

Q00, the initial boundary condition, is the component failed probability at $t=0, q(0)$. Q00 must be set equal to a value between 0.0 and 1.0 .

The initial boundary condition $\mathrm{q}(0)$ was discussed in Input Group 10. As explained in this discussion, usually $Q 00$ is read in as 0.0 since the component is known to be in the functioning state at the start of operation.

1. Continuation of the Characteristics by the Method of Translation

Generally, the only situation where $Q 00$ is not read in as 0.0 is when a problem is "continued". A computer run is made, with given input data, and the characteristics are obtained to a maximum time $t_{\max }$. The same characteristics may be continued for greater times by making a secund cumpuler run w1th all tne phase input data translated in time. In this second computer run, the time points $t_{g}$ [ie, TOT(J) or $j \Delta t$, where $\Delta t=D E L T A]$ are interpreted as being with respect to $t_{\text {max }}$; the time points $t_{\mathfrak{j}}$, input and printed out in the second computer run, are interpreted as being $t_{\max }+t_{j}$ on the time axis of the first computer run. In this same sense, the characteristics obtained in this second computer run are interpreted, not as being for the time points $t_{j}$ printed out, but for the time points $t_{\max }+t_{j}$ when translating to the time axis of the first computer run.

In order to achieve this continuation, the component phase input must be advanced in time to begin at $t_{\max }$. For the second computer run, the time axis for the phases is simply translated to begin at $t_{\max }$. The phases are renumbered and are reassigned end times with 
respect to $t_{\max }$. This translated phase data are then input to continue the characteristics in the second computer run.

Figure 11 illustrates the method of translating the phase input for a general component; the phase data must be translated in this manner for all the components. The first phase of the component begins at $t_{\max }$, the last time point from the first computer run and the origin for the second computer run. As illustrated in the figure, the phase after $t_{\max }$ with an end time of $t_{n}$ in the first run has an end time of $t_{n}-t_{\max }$ read in for the second run. The fact that the first phases of the second run do not begin at actual phase boundaries is of no significance because of the nature of the equations solved by the $\operatorname{code}^{[2]}$.

Besides the phase translations, the only other change required in the input (from the first run) is the initial boundary conditions used, Q00. In the second computer run, Q0O for each component must now be read in as having the value $q\left(t_{\max }\right)$, the failed probability for the respective component at $t_{\max }$ obtained from the first run.

With these data changes, the reliability characteristics obtained from the second computer run will merely be continuations of those obtained from the first run (with the time points $t_{j}$ and corresponding characteristics interpreted as being at $t_{\max }+t_{j}$ as explained earlier $[a])$. The differential characteristics obtained from the second run will be true continuations of the corresponding values in the first run. The integral characteristics obtained will involve integrals from $t_{\max }$ to $t_{\max }+t_{j}$. (As stated previously in Input Group 4, the integral characteristics are always evaluated from the

$\overline{[a]_{\text {The time points }}}$ used for the second run, eg, $\operatorname{TOT}(J)$, need not of course be lhe silite as biost used for the first run. 


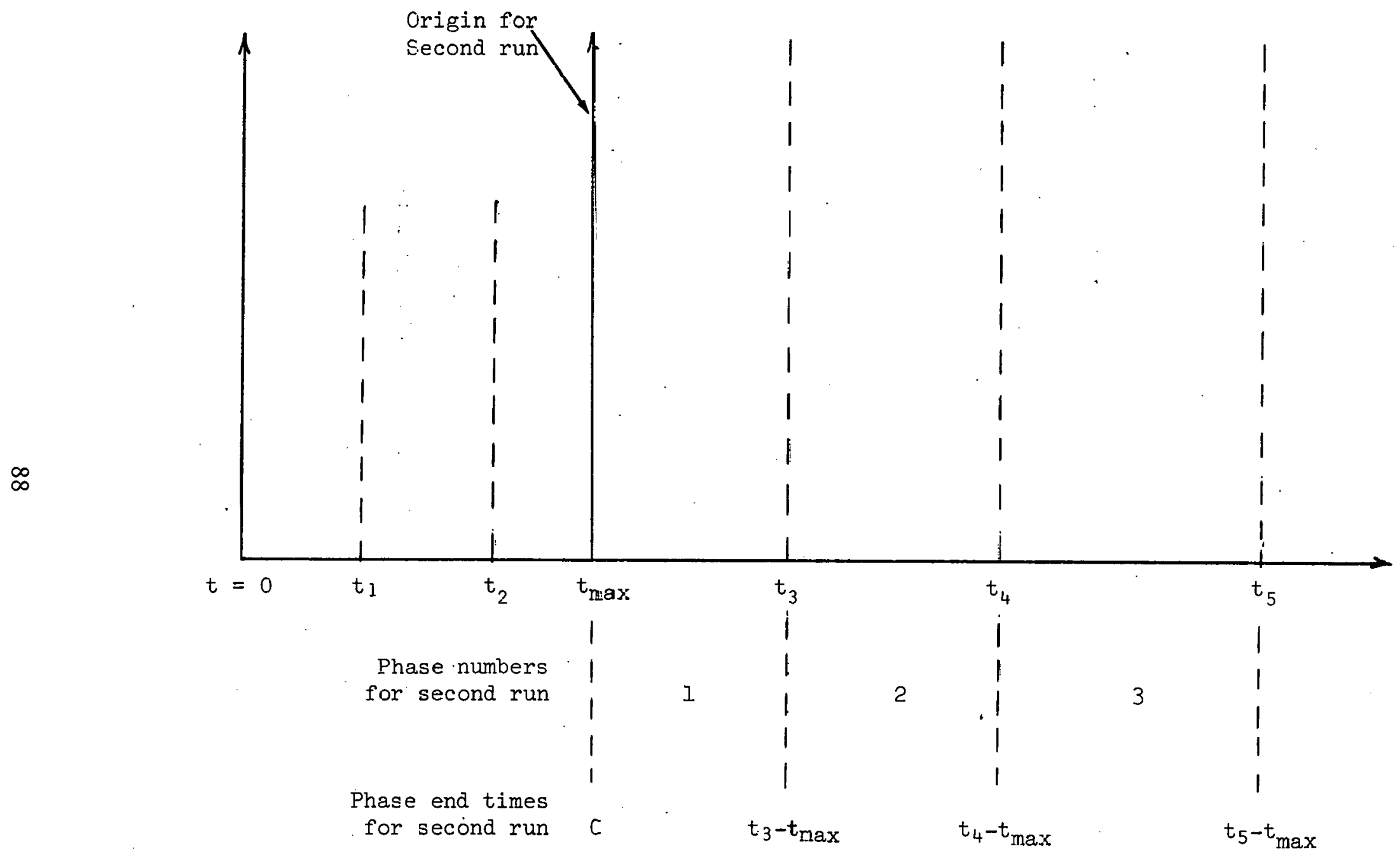

FIGURE 11. Translation of Phase Input Data 
first time point input.) However, the continuation of the integral characteristics can be obtained by simple additions and multiplications:

$$
\begin{aligned}
\operatorname{WSUM}\left(t_{\text {max }}+t_{j}\right) & =\operatorname{WSUM}\left(t_{\text {max }}\right)+\operatorname{WSUM}\left(t_{j}\right) \\
\operatorname{FSUM}\left(t_{\text {max }}+t_{j}\right) & =\operatorname{FSUM}\left(t_{\text {max }}\right)+\operatorname{FSUM}\left(t_{j}\right) \\
& -\operatorname{FSUM}\left(t_{\text {max }}\right) \cdot \operatorname{FSUM}\left(t_{j}\right)
\end{aligned}
$$

WSUM is the integral of the failure rate for either a component, a set, or the system. WSUM $\left(t_{\max }+t_{j}\right)$ is the continued value, WSUM $\left(t_{\max }\right)$ the value at $t_{\max }$ obtained from the first run and $\operatorname{WSUM}\left(t_{j}\right)$ the value obtained from the second run at $t_{j}$. FSUM is the probability of one or more failures for either a component, set, or the system: FSUM $\left(t_{\max }+t_{j}\right)$ is the continued value, FSUM $\left(t_{\max }\right)$ the value at $t_{\max }$ from the first run and $\operatorname{FSUM}\left(t_{j}\right)$ the value at $t_{j}$ from the second run. (The symbols used are the same as those output by KITT-2.)

The above continuation procedure can be repeated as many times as desired, always translating the phase data, and reading in the last component failed probability obtained as the initial boundary value $Q 00$ for the next run. In this manner, a large number of points for the reliability characteristics can be obtained--many more than is possible with just one computer run.

2. Continuation of the Characteristics by a Simple Time Point Advance

The above method of continuing the reliability characteristics is a bit laborious because the phase data must be translated betore each successive computer run. Another method of continuing the characteristics from computer run to computer run is to keep all the phase input data the same and simply use a new set of time points for each run. 
The first computer run is made with time points up to $t_{\max }$. The second computer run is made with time points beginning at $t_{\max }$ and ending at $t_{\max }(2)[a]$. The phase data for this second run are left exactly the same as for the first run, and the initial boundary conditions QOO are also left unchanged. The only input data which are different are the time points used, $\operatorname{TOT}(\mathrm{J})$.

With this simple change in input data, the results obtained from the second run are then merely continuations of the characteristic values from the first run, for the new time points used. Since the integral characteristics are always evaluated with a beginning time of $\operatorname{TOT}(1)$, the integral characteristics obtained from the second run must be incorporated with their respective values at $t_{\max }$ obtained from the first run. Equations (110) and (111) give the simple manipulations required for this incorporation.

$$
\begin{aligned}
\operatorname{WSUM}\left(0 ; t_{j}\right) & =\operatorname{WSUM}\left(t_{\text {max }}\right)+\operatorname{WSUM}\left(t_{j}\right) \\
\operatorname{FSUM}\left(0 ; t_{j}\right) & =\operatorname{FSUM}\left(t_{\text {max }}\right)+\operatorname{FSUM}\left(t_{j}\right) \\
& -\operatorname{FSUM}\left(t_{\text {max }}\right) \cdot \operatorname{FSUM}\left(t_{j}\right)
\end{aligned}
$$

WSUM is the integrated failure rate and FSUM is the probability of one or more failures--for either a component, a set, or the system. $\operatorname{WSUM}\left(0 ; t_{j}\right)$ is the continued value at a time point $t_{j}$ of the second run, WSUM $\left(t_{\max }\right)$ is the value at $t_{\max }$ obtained from the first run, and $\operatorname{WSUM}\left(t_{j}\right)$ the value at $t_{j}$ obtained from the second run. The notation is the same for FSUM. (The symbols are the same as those printed out by KII'I-2.) These simple manipulations will then give the continuation

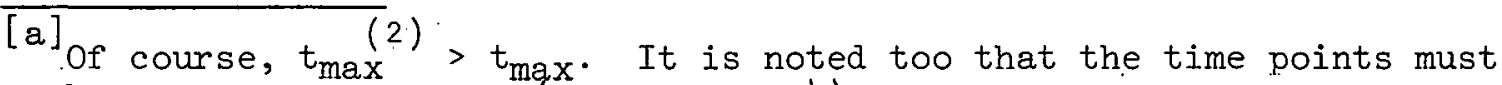
be explicitly read in (Input Group 4) since the first time point $t_{\max }$ has a value greater than zero.
} 
of the integral characteristics $\left[\operatorname{WSUM}\left(0 ; t_{j}\right)\right.$ and $\left.\operatorname{FSUM}\left(0 ; t_{j}\right)\right]$. The differential characteristics need no adjusting for their continuation.

A third computer run can be made with time points beginning at $t_{\max }(2)$ and ending at a time $t_{\max }(3)\left[\operatorname{TOT}(1)=t_{\max }(2)\right.$ and TOT(NTPT) = $t_{\max }(3)$ for this run]. Again, all the other input data are left unchanged. The results obtained are then further continuations of the characteristics. The differential characteristics are true continuations and the integral characteristics need only be incorporated with their respective values at $t_{\max }(2)$ from the second run. [In Equations (110) and (111) change $t_{\max }$ to $t_{\max }(2)$ and interpret $t_{j}$ as a time point in the third computer run.] This procedure of using an increasing set of time points in each successive computer run can be repeated as many times as desired.

This second method of continuing the reliability characteristics is simpler to use than the method of translation described earlier. However, the computer time will be longer for each successive computer run for this method. Generally, however, the increase in computer time is negligible.

The fact that the first time point, TOT(1), has an increasingly larger value for each successive run in this second method in no way affects the accuracy of the results obtained. It is only the spacing of the time points after TOT(1) which is important and, as described in Input Groups 3. and 4, the spacing only affects the accuracy of the integral characteristics obtained. This method of continuation, like the translation method, is therefore a method of obtaining exceedingly accurate results by using many time points. 
For Each Inhibit Condition, Read In The Following Three Input Groups

(Input Groups 15 Through 17). These Input Groups Are Input In Order For

Each Inhibit Condition. Repeat These Three Input Groups For As Many

Inhibit Conditions As There Are. If There Are No. Inhibit Conditions,

Skip These Three Input Groups And Go To Input Group 18.

Input Group 15 (First Group of the Inhibit Condition Group Set)

Number of Cards: 1

Format: 2110

Input Data: IDEX, NPHASE $1 \leq \mathrm{NPHASE} \leq 50$

IDEX is the negative of the inhibit condition index, and NPHASE is the number of phases for the inhibit condition.

The assigning of phases to an inhibit condition has been discussed on page 10. As stated in Input Group 8, the components and inhibit conditions are assigned consecutive indices from 1 to NCOMP for identification. This assignment is arbitrary. To differentiate the inhibit conditions, a negative sign is attached to the index of the inhibit sonnition when it ia read in, the reason IDEX is the negative of the index of the inhibit condition. Like the components, the inhibit conditions may be input in any order with respect to their indices.

Input Group 16 (Second Group of the Inhibit Condition Group Set) Number of Cards: Varies

Format: 8 F10.0

Input Data: $\operatorname{TP}(\mathrm{L}) ; \mathrm{L}=1$, NPHASE 
$\operatorname{TP}(L)$ are the phase end times (in hours) for the inhibit condition phases. The phase end times must be input in order of phase number; ie, $\mathrm{TP}(1)$ is the end time of Phase $1, \mathrm{TP}(2)$ of Phase $2, \ldots, \mathrm{TP}$ (NPHASE) of phase NPHASE. Phase $I$ is always assumed to have a beginning time of $t=0.0$. The phase end times for an inhibit condition are completely analogous to the phase end times for a component (Input Group 9). As for the component phases, the end time of the $n^{\text {th }}$ phase is always assumed to be the beginning time of the $(n+1)^{\text {th }}$ phase. The end time of the final phase for the inhibit condition TP(NPHASE) must again always be larger than the maximum time for which reliability characteristics are obtained. In this respect, the user is referred to Equations (102) through (105) on pages 80 and 81 , where "TP(NPHASE)" in those equations may be interpreted as the end time of the final phase for the inhibit condition.

Input Group 17 (Third and Last Group of the Inhibit Condition Group Set) Number of Cards: Varies

Format: $8 \mathrm{~F} 10.0$

Input Data: $\mathrm{PBOB}(\mathrm{L}) ; \mathrm{L}=\mathrm{I}$, NPHASE

$\mathrm{PBOB}(\mathrm{L})$ is the probability of occurrence for the inhibit condition for Phase $L, L=1, \ldots$, NPHASE.

In Figure 10 on page $71, \mathrm{PBOB}(L)$ corresponds to the occurrence probabili.ty $\mathrm{P} ; \mathrm{PBOB}(1)$, for example, corresponds to $\mathrm{P}_{1}$. Since the $\mathrm{PBOB}(\mathrm{L})$ are . probabilities, they must have values between 0.0 and.1.0. If for a particular phase $L, \operatorname{PBOB}(L)=0.0$, then the inhibit condition does not exist in this phase. If $\mathrm{PBOB}(L)=1.0$, then the inhibit condition is always existing in Phase $\mathrm{L}$. 
Input Group 18

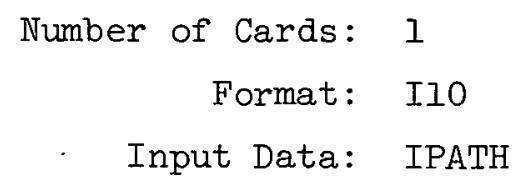

IPATH is the set flag and is read in as either 1 or 2. If IPATH $=1$, minimal cut sets are input; and if IPATH $=2$, minimal path sets are read in. (This input group corresponds to Input Group 11 of KITT-1.)

\section{Input Group 19}

Number of Cards: 1

Format: 110

Input Data: NCUT ( $I \leq N C U T \leq 500)$

NCUT is the total number of minimal cut sets input (IPATH $=1$ ) or the total number of minimal path sets input (IPATH $=2$ ). (This input group corresponds to Input Group 12 of KITT-1.)

For Fach Minimal Cut Set (IPATH $=1$ ) Or Minimal Path Set (IPATH = 2), Read In The Following Input Group (Input Group 20). Repeat This Input Group For The NCUT Total Number of Minimal Cut Sets or Path Sets.

\section{Input Group 20}

Number of Cards: Varies

Format: 8110

Input Data: $\operatorname{IMAX}, \operatorname{ICUT}(K, I) ; I=I, \operatorname{IMAX}(I \leq \operatorname{IMAX} \leq 19)$ 
IMAX is the total number of components plus inhibit conditions in the minimal cut or path set. ICUT(K,I) are the indices of the components and inhibit conditions in the set. Negative signs are not attached to the inhibit condition indices here.

This input group corresponds to Input Group 13 of KITT-1. As for that input group, the first card has as its first entry the number of components and inhibit conditions in the set. The remaining entries on the first card, up to 7 such entries, are the indices of the components and inhibit conditions in the set. If there are more than 7 indices, they are continued, 8 to a card ( $8 \mathrm{I} 10$ Format), on as many cards as necessary. The indices need not be in any particular order on the cards.

\section{FORMAT OF OUTPUT FROM KITT-2}

The format of output from KITT-2 is very similar to that of KITT-I with identical symbols used for the reliability characteristics printed out. The output of KITT-2 differs from that of KITI-1 chiefly in the way the output is grouped.

The first page of output from KITT-2 is simply a printout of the values used for Input Groups 1 through 7. This first page is selfexplanatory with the names of the variables, as used in these input discussions, being printed out along with their values. A brief description of each variable is also given to aid the user in its identification.

The second page of output is entitled "COMPONENT AND INHIBIT INFORMATION". This output group contains the data read in for each component and inhibit condition (Input Groups 8 through 14 and Input Groups 15 through 17). In addition, the reliability characteristics obtained for 
the respective components are also printed out. The information for each component is separated by a row of asterisks (*), while each particular inhibit condition is separated by a row of dollar signs ( $\$$ ).

For a particular component, again differentiated by a row of asterisks, the component index and its number of phases are first printed out. This is followed by a printout of the input data read in for that component (Input Groups 8 through 14). The input data are clearly labelled and is self-explanatory. Immediately following the input data for the component is a printout of the component reliability characteristics. The reliability characteristics are printed out for the time points specified. In addition, whenever a right boundary of a phase is reached, the characteristics are printed out for this right boundary value time. The values of the characteristics at the right boundary times (ie, at the phase end times) are boxed in asterisks to distinguish them as being boundary values. They are also labelled, giving the phase number having this time as a right boundary.

These right boundary characteristics printed out are for the time at the right boundary as this time is approached from the left; if $t_{n}$ is the time of the right boundary of the $n^{\text {th }}$ phase, then the characteristics are printed out for the time $t_{n^{-}}$.

This differentiation is needed since the only differential component. characteristic which is continuous across phase boundaries is the component failed probability,

$$
q\left(t_{n^{-}}\right)=q\left(t_{n 1}+\right)
$$

where $t_{n}+$ is the time $t_{n}$ approached from the right. This continuity of $q(t)$ is true, except when there are imposed boundary conditions (Input Group 10). The component failure rate $w(t)$ is discontinuous across the 
phase boundaries if the failure intensity $\lambda(t)$ is discontinuous across these boundaries. The integral characteristics are of course always continuous across phase boundaries.

The component characteristics, including the values at the right boundaries, are printed out in exactly the same format as used for KITT-I. For the format translation the user is therefore referred to the discussion on the "COMPONENT AND INHIBIT INFORMATION" output groups from KITT-I (page 50) or he is referred to Table D-1 of Appendix D which summarizes this translation.

Contained in the output group "COMPONENT AND INHIBIT INFORMATION", along with the component information, is the output of the inhibit condition information. This inhibit condition output is merely a printout of the input data read in (Input Groups 15 through 17). For a particular inhibit condition, differentiated by a beginning row of dollar signs ( $\$$ ), its counter, index, and number of phases are first given. These are printed out in the format "INHIBIT CONDITION $\mathrm{K}$ WITH INDEX $=\mathrm{I}$, AND NO. OF PHASES $=N^{\prime \prime}$. $K, I$, and $\mathbb{N}$ will be numbers in the actual printout. I is the index of the inhibit condition as assigned by the user (without any attached. minus sign), and $N$ is the number of phases for it (NPHASE). $K$ is the inhibit condition counter, merely an updated count of the total number of inhibit conditions which have been read in. The data read in for the inhibit condition then follow.

Immediately after the component and inhibit condition information is a printout of the minimal path or cut set information. The first of these output groups is entitled "MINIMAL SET INPUT DATA" and contains merely a printout of the set data read in (Input Groups 18 through 20). As in KITT-1, the sets are indexed in the order in which they are read in, the indices used later to reference the individual sets. 
Beyond this point the output from KITT-2 is identical both in order of printout and in format with the output from KITT-1. The output group, "MINIMAI SET INFORMATION", contains the reliability characteristics obtained for each minimal cut or path set. After this the system characteristics are printed out. Because of the identicalness with KITT-I, the reader is referred to page 51. The tables at the end of those discussions summarize the translation of the output formats. These tables may also be found in Appendix D, placed there again for the convenience of the user. 


\section{REFERENCES}

1. D. F. Haasl, "Advanced Concepts in Fault Tree Analysis", System Safety Symposium, June 8-9, 1965, Seattle: The Boeing Company, 1965.

2. W. E. Vesely, Analysis of Fault Trecs by Kinctic Tree Theory, IN-1330 (October 1969). 


\section{APPENDIX A}

\section{PREP Input}

This Appendix is a quick reference input guide to the TREBII portion of PREP. 


\section{TABLE A-1. INPUT DECK}

1. TITLE CARD

2. \{COMMENTS CARDS

3. *bDATA ( $b=$ blank column)

4. $\left\{\begin{array}{l}\text { INPUT GROUP I } \\ \text { CONTTOL INFORMATION }\end{array}\right\}$

5. END

6. \{COMMTINTS CARDS

7. *bTREE

8. $\left\{\begin{array}{l}\text { INPUT GROUP } 2 \\ \text { FAULT TREE DESCRIPTION }\end{array}\right\}$

9. END

10. \{COMMENTS CARDS

11. *bRATES

12. $\left\{\begin{array}{l}\text { INPUTT GROUP. } 3 \\ H: A \perp L U K I \text { II AND REPAIR DATA }\end{array}\right\}$

13. END 
TABLE A-2. INPUT GROUP 1

(Control Information)

Card 1 Format (5110)

Number of Cards $=2$

\begin{tabular}{|c|c|c|}
\hline Input Quantity & Card Columns & Description \\
\hline NG & $1-10$ & $\begin{array}{l}\text { Number of logical gates in the } \\
\text { fault tree. }\end{array}$ \\
\hline MIN & $11-20$ & $\begin{array}{l}\text { Smallest combinations to be tested } \\
\text { deterministically. If MIN }=0 \text {, } \\
\text { Monte Carlo simulation is used. }\end{array}$ \\
\hline MAX & $21-30$ & $\begin{array}{l}\text { Largest combinations to be tested } \\
\text { deterministically }\end{array}$ \\
\hline IDEXI & $31-40$ & $\begin{array}{l}\text { Cut set - path set switch. } \\
\text { If IDEXI = } 0 \text {, minimal cut sets are } \\
\text { found. If IDEXI = I, minimal path } \\
\text { sets are found. }\end{array}$ \\
\hline IDEX2 & $4 I-50$ & $\begin{array}{l}\text { Print-punch switch. } \\
\text { If IDEX2 = } 0 \text {, results are printed } \\
\text { only. If IDEX2 = I, results are } \\
\text { both printed and punched. }\end{array}$ \\
\hline
\end{tabular}

Card 2 Format ( $4 I 10,1 F 20.3$ ) (This card will be blank if MIN>0 on card 1 )

Input Quantity $\quad$ Card Columns

MCS

NREJEC

NTR

IREN

RAA

$$
1-10
$$

$11-20$

$21-30$

$31-40$

$41-60$
Description

Smallest minimal cut sets to be found by Monte Carlo simulation.

Number of random numbers to reject.

Number of Monte Carlo trials to be run.

Monte Carlo mixing parameter switch.

Monte Carlo mixing parameter. 
TABLE A-3. INPUT GROUP 2

(Fault Tree Description)

$$
\text { Number of Cards }=\mathrm{NG}
$$

Format for each card in Input Group 2

\section{Input Quantity}

1. Gate name (any 8 alphanumeric characters)

2. Blank.

3. Gate type ("AND" or "OR")

4. Blank

5. Number of gate inputs

6. Number of component inputs

In the following input fields all of the gates input to the gate being described. (if any) must precede the components input (if any). A maximum of $T$ lipuls per gate 1 aldowed.

7. First input name

8. Blank

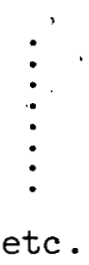

$\frac{\text { Card Columns }}{1-8 \text { (left justified) }}$
9
$10-12$ (1eft justified)
13
$14-15$ (right justified)
$16-17$ (right justified)

16-17 (right justified)
19-26 (left justified)

27

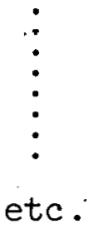




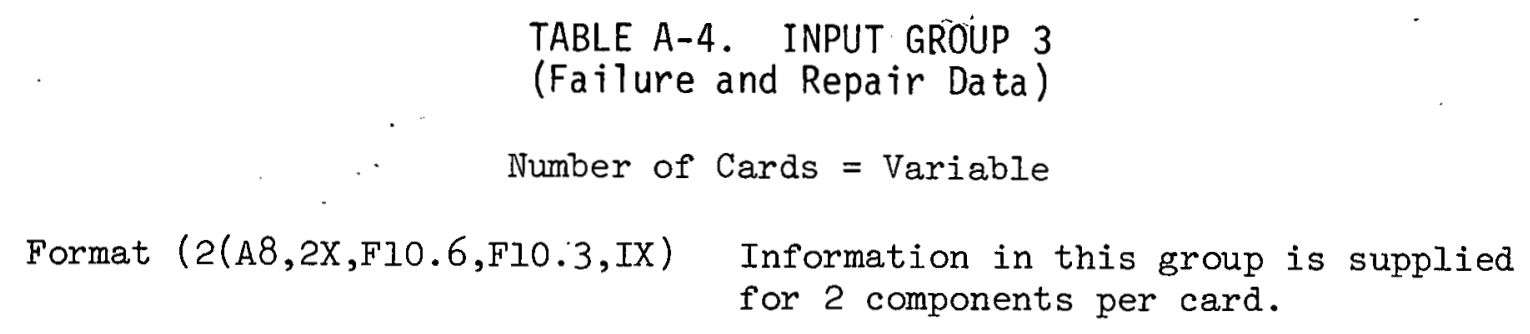

Input Quantity

1. $\operatorname{NAM}(I)$

2. Blank

3. $\operatorname{LMDA}(I)$

4. TAU (I)

5. Blank

6. $\operatorname{NAM}(J)$

7. Blank

8. $\operatorname{LMDA}(\mathrm{J})$

9. TAU $(J)$
$21-30$

Card Columns

$1-8$

$9-10$

$11-20$

31

$32-39$

$40-41$

$42-51$

$52-61$

\section{Description}

$I^{\text {th }}$ component name

Failure intensity per $10^{6} \mathrm{hr}$. ( $\lambda$ ) for the $I^{\text {th }}$ component if $\operatorname{IMDA}(\mathrm{I})>0$. If $\operatorname{IMDA}(\mathrm{I}) \leq 0$, then it is an inhibit or switch indicator.

$I^{\text {th }}$ component repair time or inhibit failure probability.

$j^{\text {th }}$ component name where $I \neq J$.

Failure intensity for $\mathrm{J}^{\text {th }}$ component.

Repair time for $J^{\text {th }}$ component. 


\section{APPENDIX B}

\section{Input for KITT-1}

The table on the following pages lists the input necessary for KITT-1, to be used as a quick reference by the experienced user. 
TABLE B-1. Input for KITT-1

(The First of Four Pages)

\begin{tabular}{|c|c|c|c|c|}
\hline $\begin{array}{c}\text { Input } \\
\text { Group No. }\end{array}$ & INo. of Cards & Format & Input Data & Information \\
\hline 1 & 1 & $10 A 8$ & Title Cara & $\begin{array}{l}\text { Any alphanumeric characters in tre } 80 \\
\text { col:umns of the card. }\end{array}$ \\
\hline 2 & 1 & II0 & NPROB & Numjer of parameter runs, $(\mathrm{NPROB} \geq 1)$. \\
\hline 3 & 1 & I10 & NCOMF & $\begin{array}{l}\text { Total number of components plus inhi- } \\
\text { bit conditions (NCOMP } \leq 400) \text {. }\end{array}$ \\
\hline 4 & Varies & $8 \mathrm{~F} 10.0$ & XLMDA: $I^{\circ} ; I=I, N C O M P$ & $\begin{array}{l}\text { Component failure intensities } \lambda_{i} \text { (per } \\
\text { hour). The ith failure intensity }\left(\lambda_{i}\right) \\
\text { corresponds with the component of in- } \\
\text { dex } i . \text { A nonpositive failure inten- } \\
\text { sity }\left(\lambda_{i} \leq 0\right) \text { denotes an inhibit con- } \\
\text { dition. }\end{array}$ \\
\hline 5 & Veries & $8 \mathrm{~F} 10.0$ & TAU (I);I=1, NCOMP & $\begin{array}{l}\text { Component repair times } \tau_{i} \text { (hours). } \\
\text { The } i \text { th repair time }\left(\tau_{i}\right) \text { corresponds } \\
\text { with the component of index } i \text {. A non- } \\
\text { positive repair time }\left(\tau_{i} \leq 0\right) \text { denotes } \\
\text { a nonrepairable component. For an } \\
\text { lnhibit condition }\left(\lambda_{i} \leq 0\right) \text {, } \tau_{i} \text { is then } \\
\text { the occurrence probability. }\end{array}$ \\
\hline 6 & 1 & Ilo & ISTOP & $\begin{array}{l}\text { Bracket flag. If ISTOP }=2 \text {, system } \\
\text { reliability characteristics are ob- } \\
\text { tained from bracketing. If ISTOP }= \\
l \text {, they are not. }\end{array}$ \\
\hline
\end{tabular}


TABLE B-1. (Contd.)

(The Second of Four Pages)

\begin{tabular}{c|c|c|c|c}
\hline $\begin{array}{c}\text { Input } \\
\text { Group No. }\end{array}$ & No. of Cards & Format & Input Data & Information \\
\hline 7 & 1 & 2II0,FI0.0 & NTPT, NOUT, DELTA & $\begin{array}{l}\text { Total number of time points at which } \\
\text { characteristics are obtained (NTPT } \leq 50), \\
\text { print out muItiple, spacing between time } \\
\text { points (in hours). If DELTA } \leq \text { 0.0, the } \\
\text { time points are read in (Input Group 8). }\end{array}$
\end{tabular}

If DELTA >0.0, skip the next input group (Input Group 8).

8

\begin{tabular}{|l|l|l|} 
Varies & $8 \mathrm{~F} 10.0$ & $\operatorname{TOT}(\mathrm{J}) ; \mathrm{J}=1, \mathrm{NTPT}$
\end{tabular}

If ISTOP $=1$, skip the nex: two input groups (Input Grcups$$
9
$$

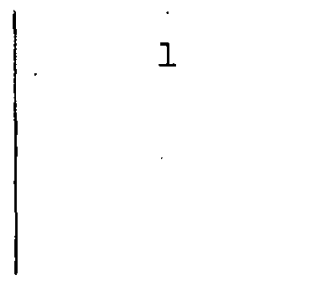

$2 \mathrm{IIO}$

NBMAX, IFAG2

If IFAG2 $=1$, skip the next input

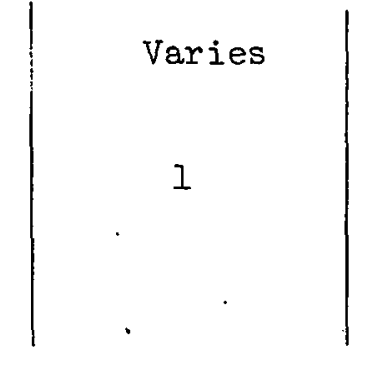

\begin{tabular}{l|l}
8110 & $\mathrm{NB} 2(\mathrm{~N}) ; \mathrm{N}=1, \mathrm{NBMAX}$ \\
$\mathrm{I} 10$ & $\mathrm{IPATH}$ \\
&
\end{tabular}

Time points (in hours) at which characteristics are cbtained. The time points must be in increasing order.

Number of outer brackets to obtain (NBMAX $\leq 400$ ), system failure rate correction flag. If IFAG2 $=2$ : the system failure correction term is computed by bracketing. If IFAG2 = 1 , this term is not computed.

Number of inner brackets for each outer bracket $(\mathrm{NB} 2(\mathbb{N}) \leq 100)$.

Set flag. If IPATH $=1$, minimal cut sets are used. If IPATH $=2$, minimal path sets are used. 
TABLE B-1. (Contd.)

(The Third of Four Pages)

\begin{tabular}{c|c|c|c|c}
\hline $\begin{array}{c}\text { Input } \\
\text { Group No. }\end{array}$ & No. cf Cards & Format & Injut Data & Information \\
\hline 12 & 1 & Ilo & NCUT & $\begin{array}{l}\text { Number of rinimal cut or path sets (NCUT } \leq \\
500) .\end{array}$
\end{tabular}

Fo: each set read in the following input group (Input Group 13).

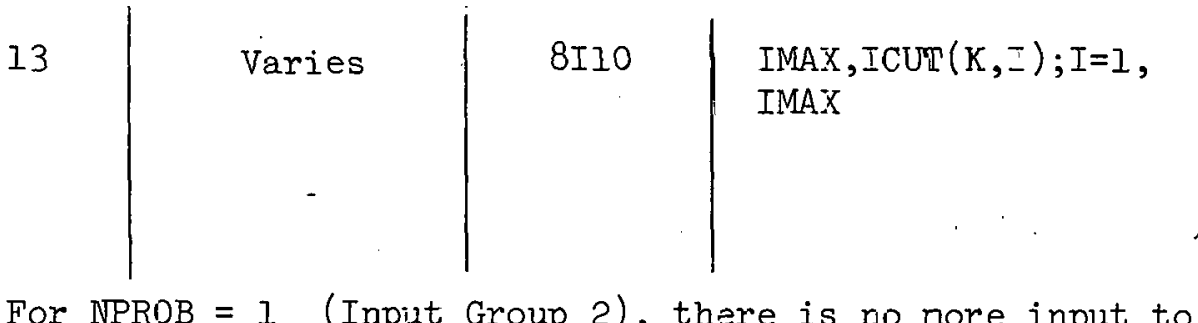

Number of components plus inhibit conditions in the set (IMAX $\leq 19$ ), list of component and inhibit condition indices in the set. For more than 7 indices, continue on the next card with the same format (8II0).

following input groups for each remaining panameter run.

$14\left|\begin{array}{l}1 \\ \text { II }\end{array}\right|$ ILAM

Number of components and inibit conditions which have different failure intensities $(\lambda)$ for the new parameter run.

If NLAM $=0$ or NLAM < NCOMF, skip the next input group (Input Group 15A).

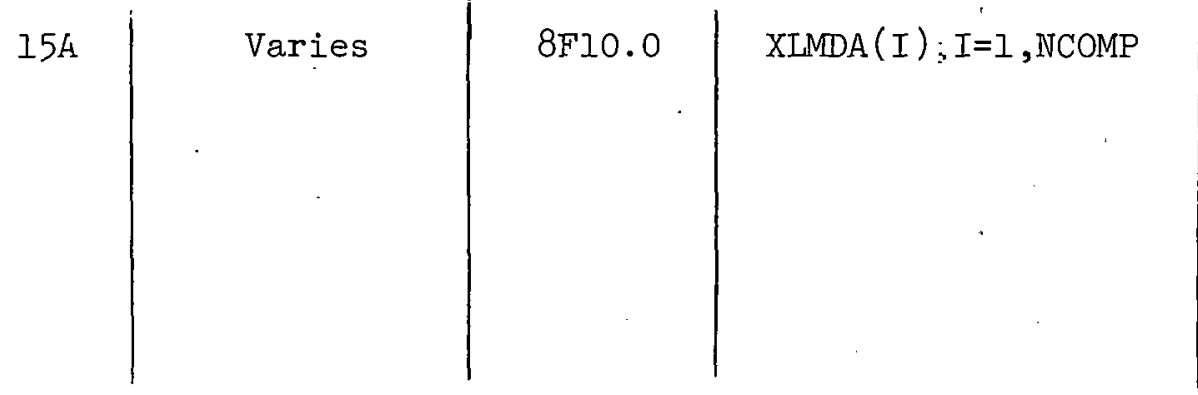

The new failure intensities (per hour) for the parameter run. The components and inhibit conditions retain the same indices as for the first parameter run (ie, as for Input Group 4). 
TABLE B-1. (Contd.)

(The Fourth of Four Pages)

\begin{tabular}{c|c|c|c|c}
\hline $\begin{array}{c}\text { Input } \\
\text { Group No. }\end{array}$ & No. of Cards & Format & Input Data & Information \\
\hline
\end{tabular}

If NLAM $=0$ or NLAM = NCOMP, skip the next input group (Input Group 15B).

\begin{tabular}{|c|c|c|c|}
\hline 15B. & Varies & $4(\mathrm{IIO}, \mathrm{F} 10.0)$ & $\begin{array}{l}\operatorname{IND}(\mathrm{K}), \mathrm{XLMDA}(\operatorname{IND}(\mathrm{K})) ; \\
\mathrm{K}=1, \operatorname{INLAM}\end{array}$ \\
\hline 6 & 1 & IIO & NTAU \\
\hline
\end{tabular}

Index of component or inhibit condition having a new failure intensity, new failure intensity (per hour). The failure intensities not changed will retain their same values as in the preceding parameter run.

Number of components and inhibit conditions which have different repair times $(\tau)$ for the new parameter run.

If NTAU $=0$ or NTAU < NCOMP, skip the next input group (Input Group 17A).

\begin{tabular}{l|l|l|l} 
& Varies & $8 \mathrm{~F} 10.0$ & $\mathrm{TAU}(\mathrm{I}) ; \mathrm{I}=\mathrm{I}, \mathrm{NCOMP}$ \\
& & &
\end{tabular}

The new repair times (hours) for the parameter run. The components and inhibit conditions retain the same indices as for the first parameter run (ie, as for Input Group 5).

If $\mathrm{NTTAU}=0$ or NTAU $=$ NCOMP, skip the next input group (Input Group I7B).

$17 \mathrm{~B}$

\begin{tabular}{|l|l|l} 
Varies & $4(\operatorname{IIO}, \mathrm{FIO} .0)$ & $\begin{array}{l}\operatorname{IND}(\mathrm{K}), \operatorname{TAU}(\operatorname{IND}(\mathrm{K})) ; \\
\mathrm{K}=1, \operatorname{ITAU}\end{array}$
\end{tabular}

Index of component or inhibit condition having a new repair time, new repair time (hours).

The repair times not changed will retain their same values as in the preceding parameter run. 


\section{APPENDIX C}

Input for KITT-2

The table on the following pages summarizes the input for KITT-2. 
TABLE C-1. Input for KLTT-2

(The First of Four Pages)

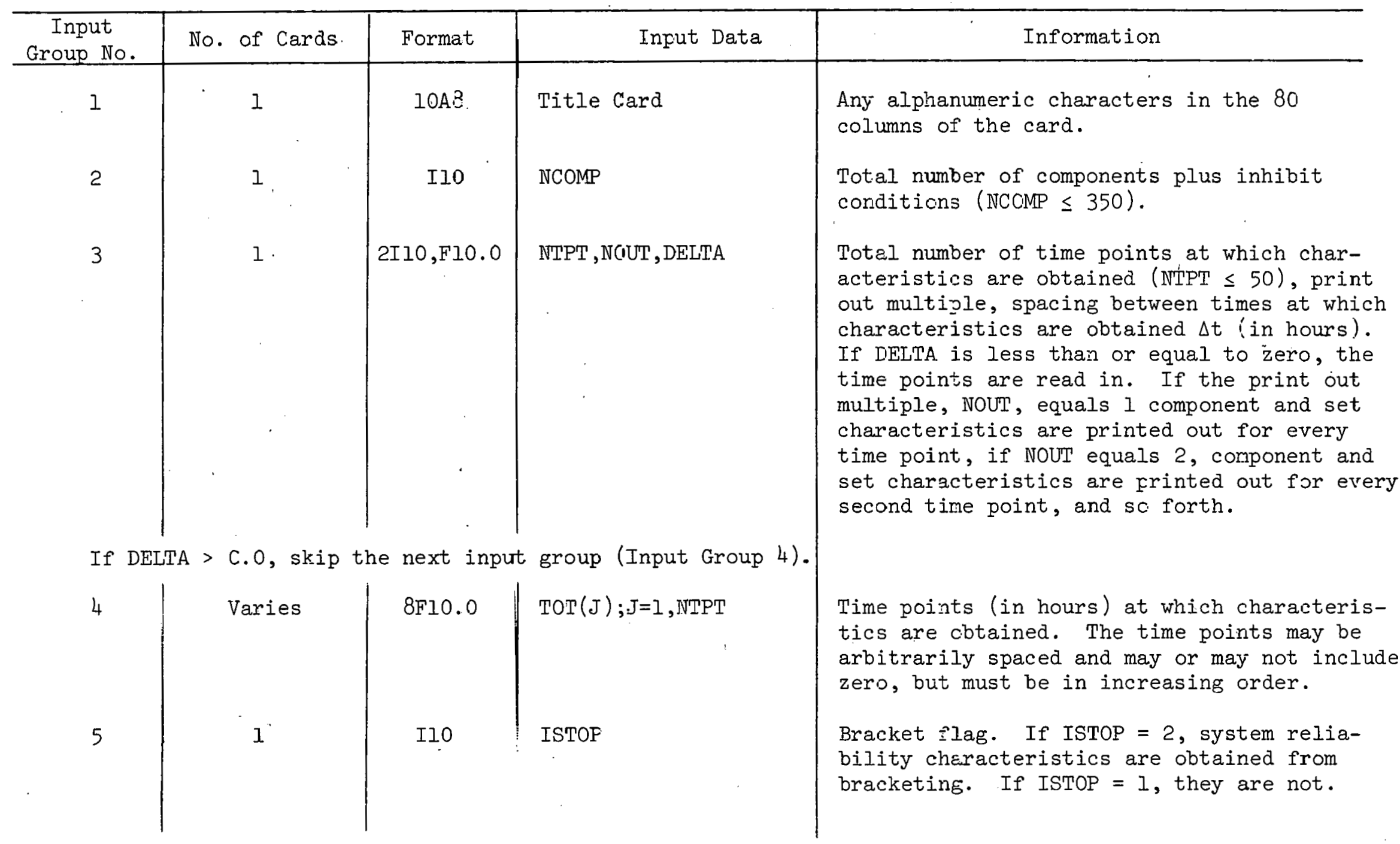


TABLE C-1. (Contd.)

(The Second of Four Pages)

\begin{tabular}{c|c|c|c|c}
\hline $\begin{array}{l}\text { Irput } \\
\text { Iroup No. }\end{array}$ & No. o: Cards & Format & Input Data & Information \\
\hline If ISTOP $=1$, skip the next two input groups (Input Groups 6 and 7).
\end{tabular}

6

\begin{tabular}{l|l|l|l}
1 & $2 I 10$ & NBMAX,IFAG2 \\
&
\end{tabular}

If IFAG $\tilde{c}=1$, skip the next input group (Input Group 7).

\begin{tabular}{l|l|l|l|l}
7 & Varies & $8 I 10$ & NB2(N);N=1,NBMAX & $\begin{array}{l}\text { Number of inner brackets for each outer } \\
\text { bracket (NB2(N) } \leq 30) .\end{array}$ \\
\hline
\end{tabular}

For each component, read in the following seven input groups (Input Groups 8 through 14). Repeat for as many somponents as there are.

8

9

10
1

Varies

1
2IIO

$8 \mathrm{~F} 10.0$

IIO
IDEX, NPHASE

. $\operatorname{TP}(L) ; I=I, N P H A S E$

IBPHA
Number of outer brackets to obtain (NBMAX $\leq 100)$, system failure rate correction flag. If IFAG $2=2$, the system failure rate correction term is computed by bracketing. If IFAG2 $=1$, this term is not computed.

Number of inner brackets for each outer

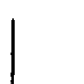

The component index, the number of phases for the component (NPHASE $\leq 50$ ).

The end-times (hours) for each phase in ' order of increasing phase number. Phase $I$ is always assumed to begin at $t=0$.

Boundary index (IBPHA $\leq 50)$ If IBPHA $=$ 0 , there are no boundary conditions imposed on the phase boundaries. If IBPHA $>0$, then IBPHA is the number of such boundary conditions. 
TABLE C-1. (Contd.)

(The Third of Four Pages)

\begin{tabular}{|c|c|c|c|c|}
\hline $\begin{array}{l}\text { Input } \\
\text { Group No. }\end{array}$ & No. of Cards & Format & Inout Data & Information \\
\hline
\end{tabular}

If IBPHA $\leq 0$, skip the next two input group; (Input Groups 11 and I2).

\begin{tabular}{|c|c|c|c|c|}
\hline 11 & Varies & $8 \mathrm{I} 10$ & I3POS $(X) ; K=I, I B P H A$ & $\begin{array}{l}\text { Phase numbers for which there are im- } \\
\text { oosed failed probabilities at the phase } \\
\text { beginning time. }\end{array}$ \\
\hline 12 & Varies & EF10.0 & $\mathrm{QBPOS}(\mathrm{K}) ; \mathrm{K}=1, \mathrm{IBPHA}$ & $\begin{array}{l}\text { Corresponding imposed failed probability } \\
\text { for phase IBPOS }(\mathrm{K}) \text {. }\end{array}$ \\
\hline 13 & Varies & $8 \mathrm{~F} 10.0$ & $\begin{array}{l}\operatorname{XLMDA}(-), \operatorname{TAU}(\mathrm{L}) ; \mathrm{L}=\mathrm{I}, \\
\text { NPHASE }\end{array}$ & $\begin{array}{l}\text { The component failure intensity } \lambda_{\mathrm{L}} \text { (per } \\
\text { hour! and repair time } \tau_{L} \text { (hours) for } \\
\text { Phase } \mathrm{L} \text {; } \mathrm{L}=1 \text {, NPHASE. If } \lambda_{\mathrm{L}}<0 \text {, the } \\
\text { component is nonrepaizable, and }-\lambda=\text { is } \\
\text { ¿ts failure intensity. If } \tau_{\mathrm{L}}<0 \text {, the } \\
\text { component has a constant repair intensi- } \\
\text { ty } \mu_{\mathrm{L}} \text { (per hour) and } \mu_{\mathrm{L}}=-1 / \tau_{\mathrm{L}} \text {. }\end{array}$ \\
\hline 14 & 1 & F10.0 & Q०० & $\begin{array}{l}\text { The initial failed probability at } t=0 \text {, } \\
q(0) \text {. }\end{array}$ \\
\hline
\end{tabular}

If there are no inhibit conditions, skip the next three irput groups (Input Groups $15-17$ ).

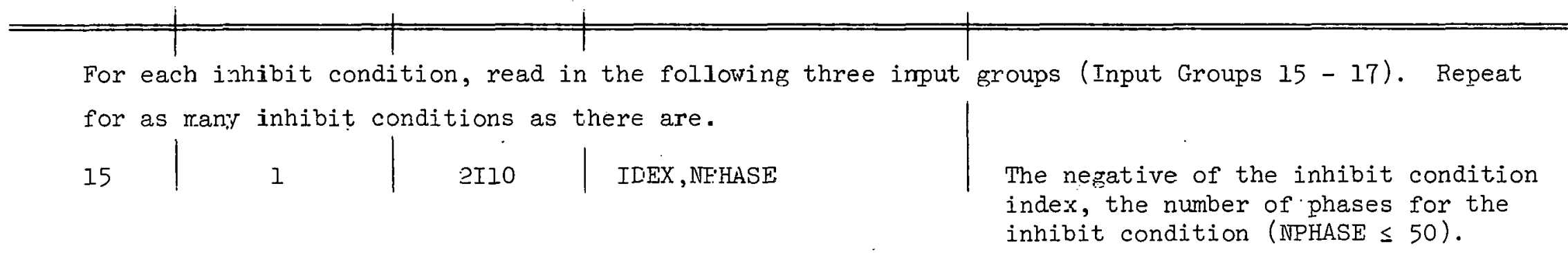


TABLE C- T. (Contd.)

(The Fourth of Four Pages)

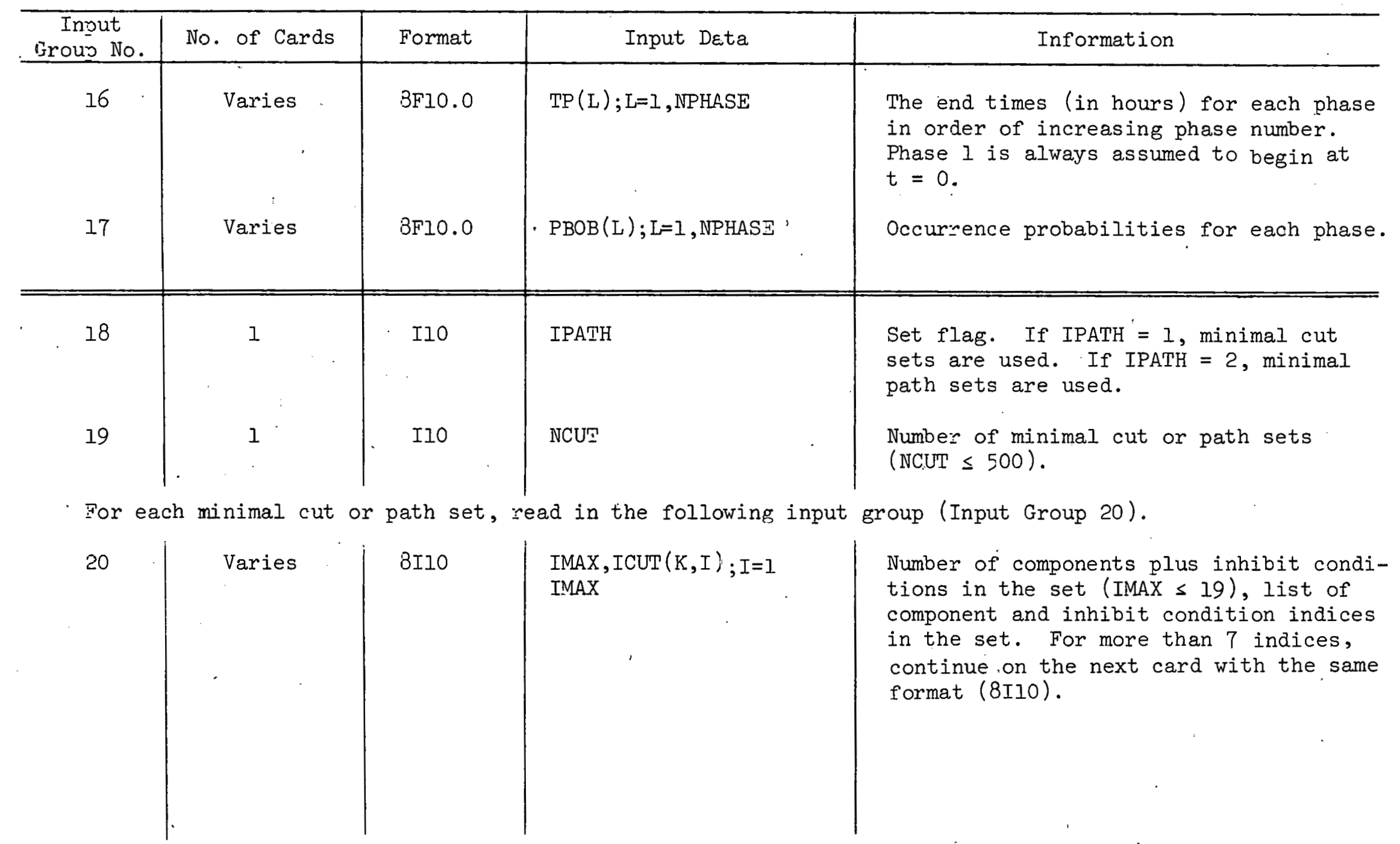




\section{APPENDIX D \\ Output From KITT-1 and KITT-2}

Tables are given here for quick translation of the KITT-I and KITT-2 output. These tables are merely a repetition of the tables given at the end of the KITT-1 discussion. They are repeated here for the convenience of the user. Since the output from KITI'I and KITT-2 is identical in format, these tables apply equally to KITT-1 and KITT-2. 
TABLE D-1

KEY TO "COMPONENT AND INHIBIT INFORMATION"

\begin{tabular}{|c|c|}
\hline Program Output & Information \\
\hline T (hours) & $t$, time (in hours) \\
\hline Q. & $\mathrm{q}(\mathrm{t})$, the component failed probability \\
\hline W & $w(t)$, the component failure rate (per hour) \\
\hline L & $\begin{array}{r}\lambda, \text { the (input) component failure intensity } \\
\text { (per hour) }\end{array}$ \\
\hline WSUM & $\begin{array}{r}\int_{0} w\left(t^{\prime}\right) d t^{\prime}, \text { the expected number of failures } \\
\text { to time } t\end{array}$ \\
\hline FSUM & $\begin{array}{r}1-\exp (-\lambda t), \text { the probability of one or more } \\
\text { failures to time } t\end{array}$ \\
\hline
\end{tabular}

TABLE D-2

KEY TO "MINIMAL SET INFORMATION" IF MINIMAL CUT SETS ARE USED

\begin{tabular}{|c|c|}
\hline Program Output & Information \\
\hline T (hours) & $t$, time (in hours) \\
\hline Q & $\check{Q}(t)$, the minimal cut set failed probability \\
\hline $\mathrm{W}$ & $\begin{array}{r}\tilde{W}(t), \text { the mininal cut set failure rate } \\
\text { (per hour) }\end{array}$ \\
\hline L & $\begin{array}{r}\check{\Lambda}(t), \text { the minimal cut set failure intensity } \\
\text { (per hour) }\end{array}$ \\
\hline WSUM & $\begin{array}{c}\int_{0} \tilde{W}\left(t^{\prime}\right) d t^{\prime}, \text { the expected number of failures } \\
t\end{array}$ \\
\hline FSUM & $\begin{array}{r}1-\exp \left[-\int_{0} \Lambda\left(t^{\prime}\right) d t^{\prime}\right], \text { the probability of one } \\
\text { or more failures to time } t\end{array}$ \\
\hline
\end{tabular}


TABLE D-3

KEY TO "MINIMAL SET INFORMATION" IF MINIMAL PATH SETS ARE USED

\begin{tabular}{|c|c|}
\hline Program Output & Information \\
\hline T (hours) & $t$, time (in hours) \\
\hline$P$ & $\begin{array}{r}\hat{P}(t) \text {, the minimal path set functioning } \\
\text { probability }\end{array}$ \\
\hline W & $\begin{array}{r}\hat{W}(t) \text {, the minimal path set failure rate } \\
\text { (per hour) }\end{array}$ \\
\hline L & $\begin{array}{r}\hat{\Lambda}(t) \text {, the minimal path set failure intensity } \\
\text { (per hour) }\end{array}$ \\
\hline WSUM & $\begin{array}{r}\int_{0}^{t} \hat{W}\left(t^{\prime}\right) d t^{\prime}, \text { the expected number of failures } \\
t \\
\text { to time } t\end{array}$ \\
\hline NO FSUM & $\begin{aligned} \exp \left[-\int_{0} \Lambda\left(t^{\prime}\right) d t^{\prime}\right], & \text { the probability of no } \\
& \text { failure to time } t\end{aligned}$ \\
\hline
\end{tabular}


TABLE D-4

\section{KEY TO "SYSTEM INFORMATION - UPPER BOUNDS"}

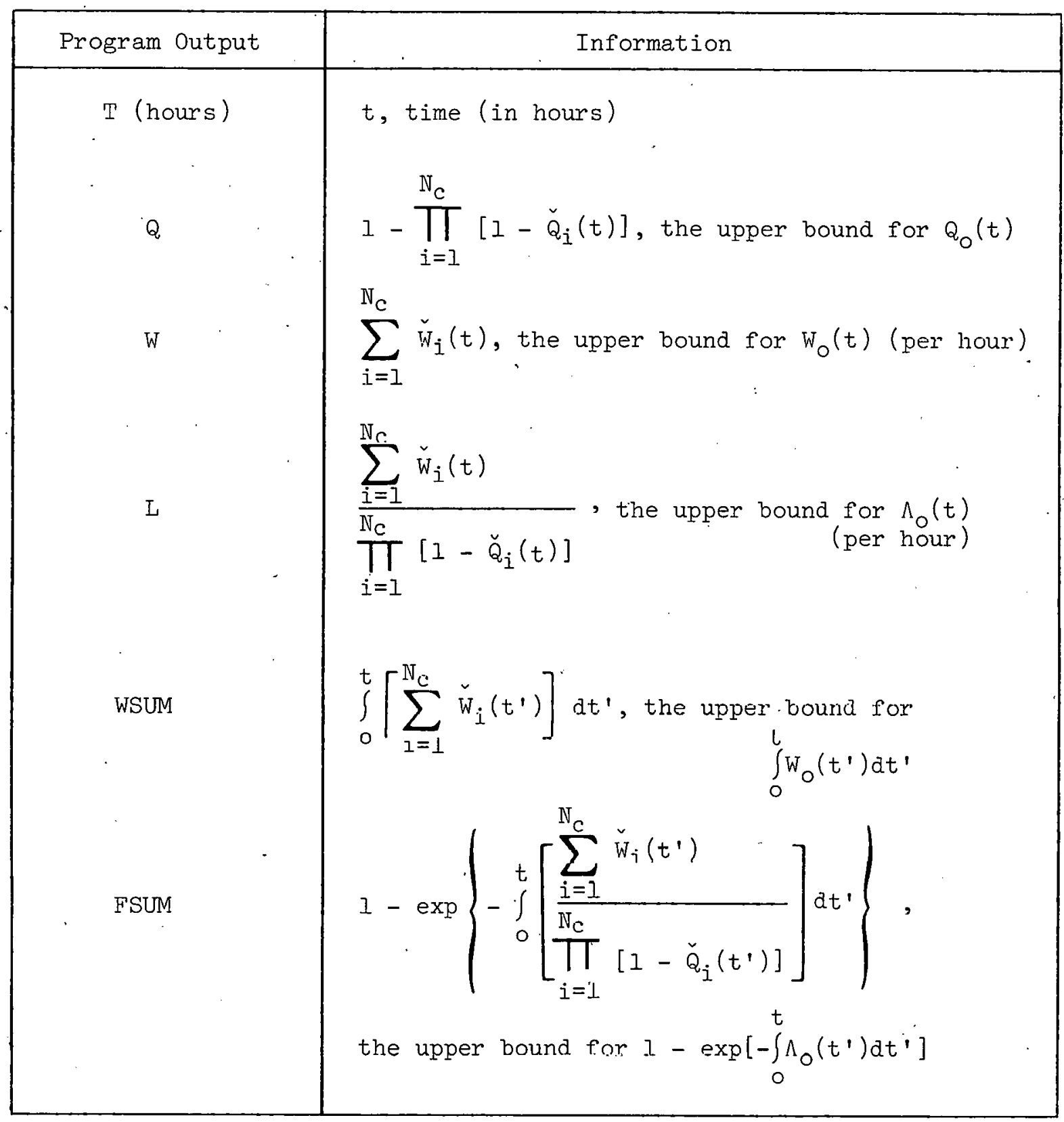


TABLE D-5

KEY TOO "DIFFERENTIAL CHARACTERISTICS - BEST BRACKETS"

\begin{tabular}{|c|c|}
\hline Program Output & Information \\
\hline QMIN & $\begin{array}{l}Q_{O}(t)_{\text {min }} \text {, the best lower bound for the } \\
\text { system failed probability } Q_{0}(t)\end{array}$ \\
\hline QMAX & $Q_{0}(t)_{\max }$, the best upper bound for $Q_{0}(t)$ \\
\hline WMIN & $\begin{array}{l}W_{0}(t)_{\min } \text {, the best lower bound for the } \\
\text { system failure rate } W_{0}(t) \text { (per hour) }\end{array}$ \\
\hline WMAX & $\begin{array}{r}W_{0}(t)_{\text {Inax }} \text {, the hest upper bound for } W_{0}(t) \\
\text { (per hour) }\end{array}$ \\
\hline LMIN & $\begin{array}{r}\Lambda_{0}(t)_{\min } \text {, the best lower bound for the } \\
\text { system failure intensity } \Lambda_{\mathrm{o}}(t) \\
\text { (per hour) }\end{array}$ \\
\hline LMAX & $\begin{array}{r}\Lambda_{0}(t)_{\max } \text {, the best upper bound for } \Lambda_{0}(t) \\
\text { (per hour) }\end{array}$ \\
\hline
\end{tabular}

TABLE D-6

KEY TO "FAILURE RATE CONTRIBUTIONS" IF MINIMAL CUT SETS ARE USED

\begin{tabular}{|c|c|}
\hline Program Output & Information \\
\hline WIMIN & $\check{W}_{0}^{(1)}(t)_{\min }$, the best lower bound for $\check{W}_{0}^{(1)}(t)$ \\
\hline WIMAX & $\begin{array}{r}\check{W}_{0}^{(1)}(t)_{\max } \text {, the best upper bound for } \check{\mathrm{W}}_{\mathrm{O}}^{(1)}(t) \\
\text { (per hour) }\end{array}$ \\
\hline$W Z M \perp N$ & $\begin{array}{r}\check{w}_{0}^{(2)}(t)_{\text {min }} \text {, the best lower bound for } \tilde{w}_{0}^{(2)}(t) \\
\text { (per hour). }\end{array}$ \\
\hline W2MAX & $\check{\mathrm{W}}_{0}^{(2)}(t)_{\max }$, the best upper bound for $\check{\mathrm{W}}_{0}^{(2)}(t)$ \\
\hline MIN and WMAX & best bounds for $W_{0}(t)$, repeated from Table $D-$ \\
\hline
\end{tabular}


TABLE D-7

KEY TO "FAILURE RATE CONTRIBUTIONS" IF MINIMAL PATH SETS ARE USED

\begin{tabular}{|c|c|}
\hline Program Output & Information \\
\hline WIMIN & $\begin{array}{r}\hat{W}_{0}^{(1)}(t)_{\min } \text {, the best lower bound for } \hat{W}_{0}^{(1)}(t) \\
\text { (per hour) }\end{array}$ \\
\hline WIMAX & $\begin{array}{r}\hat{W}_{0}^{(1)}(t)_{\max } \text {, the best upper bound for } \hat{W}_{0}^{(1)}(t) \\
\text { (per hour) }\end{array}$ \\
\hline W2MIN & $\hat{\mathrm{W}}_{\mathrm{O}}^{(2)}(t)_{\min }$, the best lower bound for $\hat{W}_{\mathrm{O}}^{(2)}(t)$ \\
\hline W2MAX & $\begin{array}{r}\hat{\mathrm{W}}_{\mathrm{O}}^{(2)}(t)_{\max } \text {, the best upper bound for } \hat{\mathrm{W}}_{\mathrm{O}}^{(2)}(t) \\
\text { (per hour) }\end{array}$ \\
\hline MIN and WMAX are & best bounds for $W_{0}(t)$, repeated from Table $D$ \\
\hline
\end{tabular}

TABLE D-8

KEY TO "DIFFERENTIAL CHARACTERISTICS - LAST BRACKETS" IF MINIMAL CUT SETS ARE IISED

\begin{tabular}{|c|c|}
\hline Program Output & Information \\
\hline If the exact vo & for system characteristics are obtained: \\
\hline QLAST & $\begin{array}{r}Q_{0}(t), \text { the exact value for the system failed } \\
\text { probability }\end{array}$ \\
\hline $\mathrm{W} I \mathrm{~L} \Lambda \mathrm{ST}$ & $\check{W}_{0}^{(l)}(t)$, its exact value (ver hour) \\
\hline$\left\{\begin{array}{l}\text { W2MIN-LAST } \\
\text { WPMAX-LAST }\end{array}\right\}$ & $\check{W}_{0}^{(2)}(t)$, its exact value (per hour) \\
\hline
\end{tabular}


TABLE D-9

KEY TO "DIFFERENTIAL CHARACTERISTICS - LAST BRACKETS"
IF MINIMAL PATH SETS ARE USED

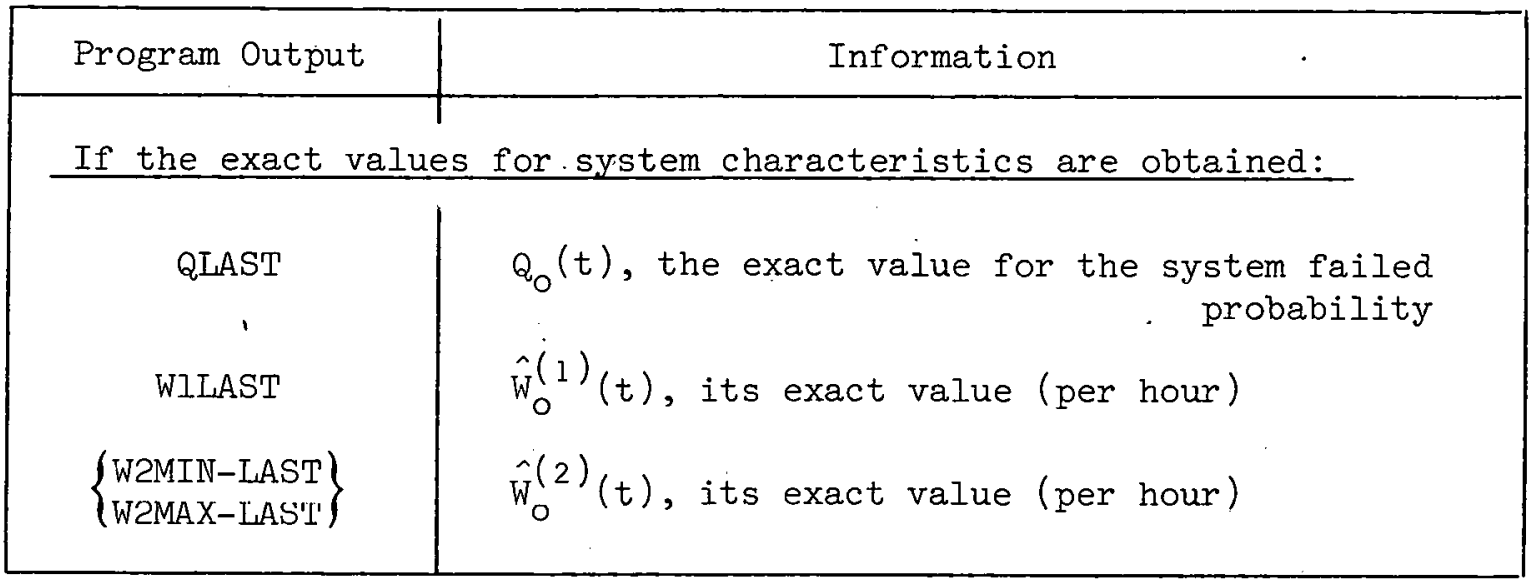

TABLE $\quad 0-10$

KEY TO "INTEGRAL CHARACTERISTICS - BEST BRACKETS"

\begin{tabular}{|c|c|}
\hline Program Output & Information \\
\hline $\begin{array}{l}\text { FMIN-SUM } \\
\cdots\end{array}$ & 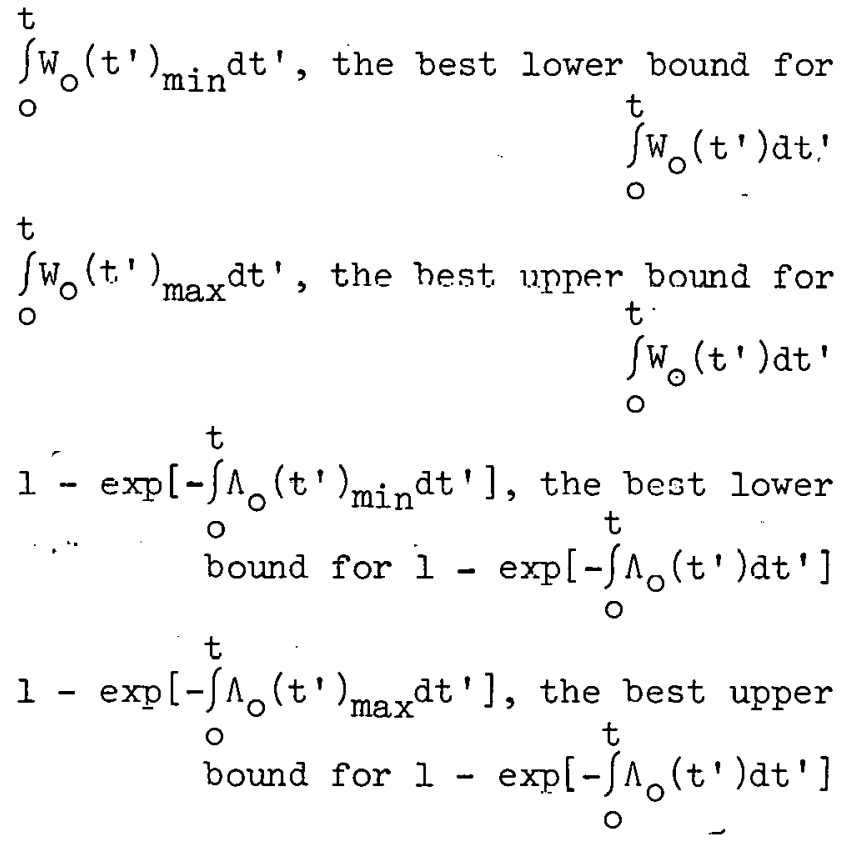 \\
\hline
\end{tabular}


APPENDIX E

The Use of the PREP and KITT Codes for Non-Time-Dependent Problems

In certain analyses, the top of the fault tree represents a certain event, and the fault tree itself represents the cause relationship of this top event to the primary events. The "components" of the fault tree are the primary events and the top of the tree is the event caused by the primary events. For example, the top of the tree may represent an occurrence of a particular type of accident, or it may represent a system failure occurring in a given experiment. For this type of fault tree, time dependence has no meaning. One wishes merely to obtain the probability of the top event occurring from knowledge of the probabilities of the primary events occurring. The PREP and KITT codes can be used in a straightforward manner to obtain the probability of this top event occurring.

For this type of fault tree, the minimal cut sets obtained by PREP are the "occurrence modes" by which the top event occurs. In a complementary manner, the minimal path sets are the "nonoccurrence modes" by which this top event does not occur. An occurrence mode consisting of a certain number of primary events means that if these primary events simultaneously occur, then the top event occurs. A nonoccurrence mode consisting of primary events means that if these primary events do not occur, then the top event does not occur. An occurrence mode therefore corresponds precisely to a minimal cut set while a nonoccurrence mode corresponds precisely to a minimal path set.

Consequently, in PREP, if occurrence modes are desired, then one should read in the input variable IDEXI such that minimal cut sets are 
found (IDEXI = 0). If nonoccurrence modes are desired, then the input variable IDEXI is read in such that minimal path sets are found (IDEXI =I). The only other input variables for PREP which need redefining are the component failure intensities $\lambda[\mathrm{LMDA}(I)]$ and the repair times $[\mathrm{TAU}(I)]$. For every component, where a component now corresponds to a primary event, read in the repair time $\mathrm{TAU}(\mathrm{I})$ as 0.0 (or leave it blank),

$$
\mathrm{TAU}(I)=0.0 \text {, for all components . }
$$

For each component (primary event) read in the failure intensity [IMDA(I)] as

$$
\operatorname{LMDA}(I)=10^{6} \mathrm{P}_{\mathrm{i}}
$$

where $P_{i}$ is the probability of occurrence for the particular primary event[a]. Except for these changes, the rest of the input data remains exactly the same and retains their meanings as defined.

Having obtained the occurrence or nonoccurrence modes (minimal cut sets or path sets), KITT-I is then used to obtain the probability of the top event occurring. In KITT-1 as in PREP, the occurrence modes correspond to minimal cut sets and nonoccurrence modes correspond to minimal path sets. For KITT-1, each primary event is treated as an inhibit condition with the probability of occurrence equal to $P_{i}$, the probability of occurrence for the particular primary event. Therefore, the failure intensities $\lambda$ read in for Input Group 4 are read in with values less than or equal to 0.0 (or left blank),

$$
\lambda_{i} \leq 0.0 \text {, for all components (primary events). }
$$

The repair times $\tau_{i}$ (Input Group 5) are read in as the respective occurrence probabilities $P_{i}$, for the primary events,

\footnotetext{
[a] The $10^{6}$ factor is needed in Equation (E-2) since PREP multiplies the failure intensities by $10^{-6}$.
} 


$$
\tau_{i}=P_{i} \text {, for each component (primary event). }
$$

If PREP is used to obtain the occurrence or nonoccurrence modes, and values were read into PREP as given by Equations (E-1) and (E-2), then the failure intensities and repair times punched out by PREP need merely be interchanged. The failure intensities used for PREP and punched out by it now become the repair times for KITT-I (Input Group 5). The repair times read into PREP as 0.0 (or left blank) now become the failure intensities for KITT-1 (Input Group 4).

Beside the primary events being interpreted as inhibit conditions for KITT-1, the only other input data which need be modified is Input Group 7. Input Group 7 contains NTPT, NOUT, and DELTA. NTPT should be read in as 2. If a printout is desired of the occurrence or nonoccurrence mode information, then set NOUT equal to I (or equal to 2). If no printout is desired of the mode information, set NOUT equal to 3 . DELTA can be read in as any positive (nonzero) number, such as 1.00 . These input values are needed for Input Group 7 because of the mechanics of the code. (Note that Input Group 8 is skipped since DELTA is read in as a positive number.) Beside Input Group 7, all other Input Groups remain exactly as previously described.

If occurrence modes are input to KITT-I to obtain the probability of the top event occurring, the failed probabilities Q printed out by KITT-I for each minimal set (occurrence mode) are the probabilities that the respective modes will occur. (If NOUT $=1$, the failed probabilities will bc printcd out for two "timc points" and will be equal. Tither value is the exact probability.) If nonoccurrence modes are input to KITT-I to obtain the probability of the top event occurring, the functioning probabilities $\mathrm{P}$ printed out for each minimal set (nonoccurrence mode) are 
the probabilities that the respective modès will not occur. Again, two such probabilities (equal in value) will be printed out if NOUT $=1$. From these failed or functioning probabilities, the most important occurrence or nonoccurrence modes are obtained. All other minimal set (mode) information will have no meaning.

For the system, the failed probability $Q$ printed out by KITT-1 is the probability that the top event will occur. Two such values will be printed out, and either one (both being equal) is that probability. All other system information printed out will have no meaning.

As stated previously, except for Input Group 7, all other input to KITT-I remains the same, with a minimal cut set corresponding to an occurrence mode and a minimal path set corresponding to a nonoccurrence mode. If an upper bound is only desired for the probability of occurrence for the top event, ISTOP (Input Group 6) is set equal to 1 . Q of the system upper bound information will then be this upper bound. If the probability of the top event is bracketed, QMIN and QMAX will then be the lower and upper bounds for, it.

If bracketing is done, IFAG2 should be set equal to I, since IFAG2 does not influence the failed probability, ie, the probability of the top event occurring. If the exact probability of the top event occurring is desired, then NBMAX of Input Group 9 should be set equal to NCUT, the total number of occurrence (or nonoccurrence) modes. If this is done, then QLAST is the exact probability. As recommended before, NBMAX should be set equal to 2 if occurrence modes are used (IPATH $=1$ ) and NCUT > 10 . 
APPENDIX F

SAMPLE FAULT TREE EVALUATION

FI 


\section{PREP Run For The Sample Tree}

The fault tree in Figure 1 was analyzed using the PREP and KITT computer programs. The PREP codes were first used to obtain the minimal cut sets of the tree, and the KITT codes were then used to obtain the associated probability characteristics.

A listing of input cards for PREP are shown on pages F3 and F4. The first of these decks is the input used to obtain the minimal cut sets from deterministic testing (COMBO). The second deck contains the necessary modifications to obtain minimal cut sets using Monte Carlo simulation (FATE). In using the deterministic testing algorithm, MIN was set to 1 . and MAX to 10. Since the output was to be punched for use by KITT, IDEX2 = 1. Pages F5 through Fl2 are the resulting output from the PREP codes. The Monte Carlo run was made using 1000 trials $(\mathrm{NTR}=1000$ ) and the mixing . parameter switch IREN set to I (uniform mixing). 'Pages F10 through F12 show those portions of the output from PREP, obtained with Monte Carlo simulation, which differ from those obtained using deterministic testing. All other output was identical to that obtained using COMBO. 
ten COMPONENT SAMPLE PROBLEM

- oata

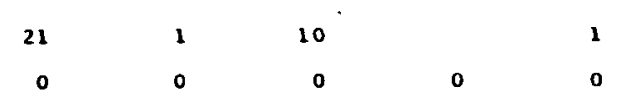

END

* tree

IUP OR 2 O GATEl gatelz

GATEI OR 2 O GATEZ GATE3

GATE3 OR 2 O GATE? Gates

GATE13 OR 2 O GATE14 GATEIS

GATE 2 OR 3 O GATEA GATES GATEG

GATE14 OR 3 O GATE 16 GATEIT GATFIB

GATE15 AND 2 O GATEI9 GATEZ

GATE $B$ AND 20 GATE 9 GATElO

GATEIO OR 2 O GATEII GATE12

GATE 7 AND 12 GATEZO COMPI COMPZ

GATE4 AND 02 COMPI COMP2

GATES AND 02 COMPI COMP3.

GATEO AND O 2 COMPZ COMP3

GATE16 AND O 2 COMPA COMP5

GATELT AND O 2 COMP4 COMPG

GATE18 ANO 02 COMP5 COMPO

GATE19 OR O 2 COMPT COMPB

GATE2O OR O 2 COMPG COMPIO

GATE9 OR 03 COMP7 COMPB COMPQ

GATE11 ANO 03 COMP1, COMPZ COMP3

- GATE 12 ANU 03 CUMPA CUMP5 COMPG

\section{END}

* rates

COMPI

COMP 3

COMPS

COAPT

cunpg -

END

$\begin{array}{rrrr}2.6 & 100.0 \text { COMP2 } & 2.0 & 100.0 \\ 2.6 & 100.0 \text { COMP4 } & -35.0 & 100.0 \\ 35.0 & 100.0 \text { COMP6 } & 35.0 & 100.0 \\ 5.0 & 100.0 \text { COMPB } & 5.0 & 100.0 \\ 8.0 & 100.0 \text { COMP10 } & 8.0 & 100.0\end{array}$


TEN COMPONENT SAMPLE PROBLEM

- data

$\begin{array}{rrrrrr}21 & 0 & 0 & 0 & 1 & \\ 1 & 100 & 1000 & 1 & & 0.0\end{array}$

END

- tree

UR 2 O GATEI GATEI3

GATEL OR 2 O GATEZ GATE 3

GATE 3 OR 2 O GATET GATEB

GATE13 OR 2 O GATELG GATELS

GATE2 OR 3 O GATE4 GATE5 GATEG

GATE 14 OR 3 O GATEL GATEIT GATELB

Gatel5 aNO $20^{\circ}$ Gatelg Gatez

GateB anO 2 O GATEQ GaTELO

GATEIO OR 2 O GATEIL GATEI2

Gate7 and 12 catezo compi compz

GATE4 AND 02 COMP1 COMP2

GATES ANO 02 COMPI COMP 3

GATE 6 ANO 02 COMP2 COMP3

GATEIG AND 02 COMPA COMPS

GATEIT AND 02 COMPA COMPO

GAIE 18 ANU 02 COMPS COMPO

GATE19 OR 02 COMPT COMPQ

GATEZO OR O 2 COMPQ COMPIO

GATES OR O 3 COMPT COMPB COMPG

GATELI ANO 03 COMPL COMP2 COMP 3

GATE 12 ANO 03 COMPA CUMPS COMPG

END

- rates

$\begin{array}{lrlrr}\text { COMPL } & 2.6 & 100.0 \text { COMP2 } & 2.6 & 100.0 \\ \text { COMP } 3 & 2.6 & 100.0 \text { COMP4 } & 35.0 & 100.0 \\ \text { COMP5 } & 35.0 & 100.0 \text { COMPS } & 35.0 & 100.0 \\ \text { COMP7 } & 5.0 & 100.0 \text { COMPB } & 5.0 & 100.0 \\ \text { COMP9 } & 0.0 & 100.0 \text { COMP10 } & 8.0 & 100.0\end{array}$

ENO 
* "TREBIL FAULT TREE BUILOING PROGRAM

**********************************************

TEN CUMPONENT SAMPLE PROBLEM

NUMBER OF GATES,NG-

COMBO STARTING VALUE, MIN---

COMBO ENDING VALUE, MAX-_._- 10

CUT SET - PATH SET SHITCH,IDEX1-_- 0

PRJNT - PUNEH SHITCH,IDEX2---1

MONTE CARLO STARTER, MCS---

NO. OF RANDOM NUMBERS TO REJECT, NREJEC-- - 0

NO. DF MONTE CARLO TRIALS, NTR--

MIXING PARAMETER SWITCH, IREN--_-_-_- -

MONTE CARLO MIXING PARAMETER, TAA--

TEN COMPONENT SAMPLE PROBLEM

\begin{tabular}{|c|c|c|c|c|c|}
\hline \multirow{2}{*}{\multicolumn{6}{|c|}{ INPUYS-- }} \\
\hline $\begin{array}{l}\text { TOP } \\
\text { GATEI }\end{array}$ & $\begin{array}{l}\text { OR } \\
\text { OR }\end{array}$ & & & & \\
\hline GATE 3 & OR & & $\begin{array}{l}\text { GATE2 } \\
\text { GATE? }\end{array}$ & $\begin{array}{l}\text { GATES } \\
\text { GATEB }\end{array}$ & \\
\hline GATE 13 & OR & & GATE14 & GATE1S & \\
\hline GATEZ & OR & & & & GATE 6 \\
\hline GATE 14 & OR & & GATE 16 & $\begin{array}{l}\text { GAIE17 } \\
\text { GIE? }\end{array}$ & GATEI8 \\
\hline $\begin{array}{l}\text { GATE } 15 \\
\text { GATE } 8\end{array}$ & $\begin{array}{l}\text { ANO } \\
\text { AND }\end{array}$ & & GATEQ & $\begin{array}{l}\text { GATEZ } \\
\text { GATE1O }\end{array}$ & \\
\hline GATE IO & OR & & GATEII & GATEI & \\
\hline $\begin{array}{l}\text { GATE } 7 \\
\end{array}$ & AND & & GATE2O & COMP1 & COMP 2 \\
\hline $\begin{array}{l}\text { GATE } 4 \\
\text { GATE }\end{array}$ & $\begin{array}{l}\text { AND } \\
\text { AND }\end{array}$ & & $\begin{array}{l}\text { COMPP } 1 \\
\text { CUMPP }\end{array}$ & $\begin{array}{l}\text { COMP2 } \\
\text { COMPP }\end{array}$ & \\
\hline GATE 6 & AND & & COMPZ & COMP3 & \\
\hline $\begin{array}{l}\text { GATE16 } \\
\text { SATE }\end{array}$ & $\begin{array}{l}\text { AND } \\
\text { AND }\end{array}$ & 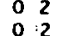 & $\begin{array}{l}\text { COMP4 } \\
\text { COMPQ }\end{array}$ & $\begin{array}{l}\text { COMPS } \\
\text { СОMРО }\end{array}$ & \\
\hline GATE IB & AND & 0 & COMPS & COMPG & \\
\hline GATE I9 & OR & 0 & COMP 7 & COMPB & \\
\hline $\begin{array}{l}\text { GATE } 20 \\
\text { GATE }\end{array}$ & $\begin{array}{l}\text { OR } \\
\text { OR }\end{array}$ & $\begin{array}{ll}0 & 2 \\
0 & 3\end{array}$ & $\begin{array}{l}\text { COMP } \\
\text { COMP } 7\end{array}$ & $\begin{array}{l}\text { СОМР10 } \\
\text { СОМРВ }\end{array}$ & \\
\hline GATE II & AND & 03 & COMPI & COMP2 & COMP 3 \\
\hline GATEIZ & AND & & COMP4 & COMPS & COMPO \\
\hline & & & & & \\
\hline
\end{tabular}


TEN COMPONENT SAMPLE PFOBLEM

THIS IS THE SLBROUTINE GENERATED BY TREBIL

LOGICAL TOP, A $200 \mathrm{C}), \times(2000$

COMMON/TREES/A, $X$, TOP

$\begin{array}{lll}\text { A1 } \quad 11=x 1 & 11 \text {-AND. } x 1 & 21 .-A N D . x 1 \\ \text { AI } & 31=x 1\end{array}$

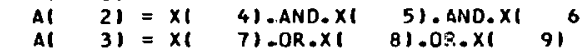

Al $41=x 1$ 9).0R.xi 101.0 .

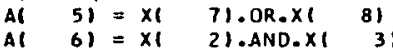

A1 $\quad 71=x 1$ 1).AND. $x 1 \quad 31$

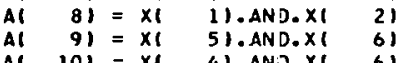

Al $111=x(11$ 4).AND. $x($ 5)

Al $121=$ Al 41 .AND.XI 41 .AND.X1 5

Al $131=$ a
Al 14$)=A($ 2). DR.Al 11

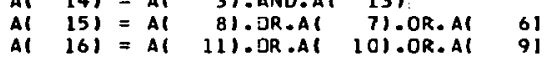

Al $161=A l$ lll. DR.Al 10$) .0 R$. Al

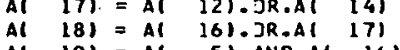

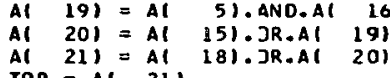

TOP $=$ AT
RETURN
END

THERE HERE 10 COMPONE VIS INDEXED IN THIS TREE

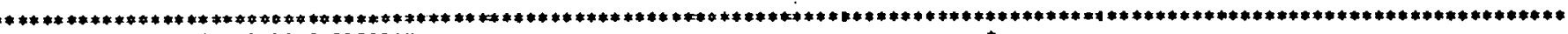
-TREBIL FAULT TREE BUILJITG PROGRAH

TEN COMPONENT SAMPLE PROBLEM

COMPONENT INDICES, names, and faIlURe RATES (PER hOUR) -

\begin{tabular}{|c|c|c|c|}
\hline $\begin{array}{c}\text { TREE INOEX } \\
1 \\
2 \\
3 \\
4 \\
5 \\
6 \\
6 \\
7 \\
8 \\
9 \\
10\end{array}$ & $\begin{array}{l}\text { COMPONENT HAME } \\
\text { COMP4 } \\
\text { COMPS } \\
\text { COMPS } \\
\text { COMPI } \\
\text { COMP2 } \\
\text { COMP3 } \\
\text { COMP7 } \\
\text { COMPA } \\
\text { COMPO } \\
\text { COMPIO }\end{array}$ & $\begin{array}{l}\text { LAMBDAIFA ILURE INIENSITY/HR . I } \\
\text { 3.50000D-05 } \\
3.500000-05 \\
3.500000-05 \\
2.600000-06 \\
2.600000-06 \\
2.600000-06 \\
5.000000-06 \\
5.000000-06 \\
8.000000-06 \\
8.000000-06\end{array}$ & $\begin{array}{l}\text { TAU } \\
100.000 \\
100.000 \\
100.000 \\
100.000 \\
100.000 \\
100.000 \\
100.000 \\
100.000 \\
100.000 \\
100.000\end{array}$ \\
\hline
\end{tabular}


TEA COMPQNENT SAMPLE PRoBLEM

TREe INDEX gate name InPUTS

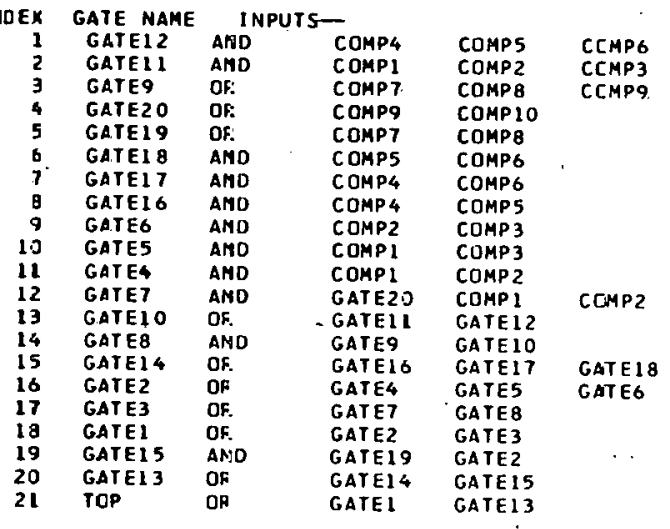

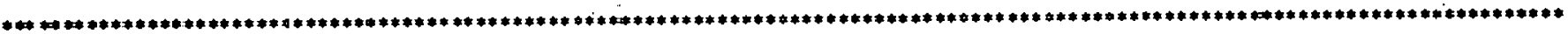
TREB IIL FAULT TREE BUILDING PROGRAM*

TE***A COMPONENT SAMPLE PROBLEM

TREE I NDEX COMPONENT NAME

NUMBER OF GATES INPUT GATES INPUT BY THIS COMPONENT

$$
\begin{aligned}
& \text { COMPL } \\
& \text { COMPS } \\
& \text { COMPS } \\
& \text { COMP } \\
& \text { COMP } \\
& \text { COMP3 } \\
& \text { COMPY } \\
& \text { COMPB } \\
& \text { COMPP } \\
& \text { COMPIO }
\end{aligned}
$$

WABR OF GATES INPUT GATE12 GATEI7 GATE16

3 GATE12 GATELI7 GATE16

gatell gates gateq gate

4 GaTEII GATEG GATE4

GATE11 GATEG

2 GATE9 GATE19

\begin{tabular}{ll}
2 & GATE9 \\
\hline & GATE9 GATELO
\end{tabular}

GATE 4 GATE?

$-\quad 8$
9
10 
LEVEL 18 ISEPT 69 ।

OS/360 =ORTRAN H

DATE $70.191 / 17.22 .31$

COMPI LER OPTIONS - NAMEF MAIN, OPT=02, LINECNT =58, SOURCE, EBCDIC, NOLI ST, NODECK, LOAD, NOMAP, NOEDIT, NOI D, NOXREF

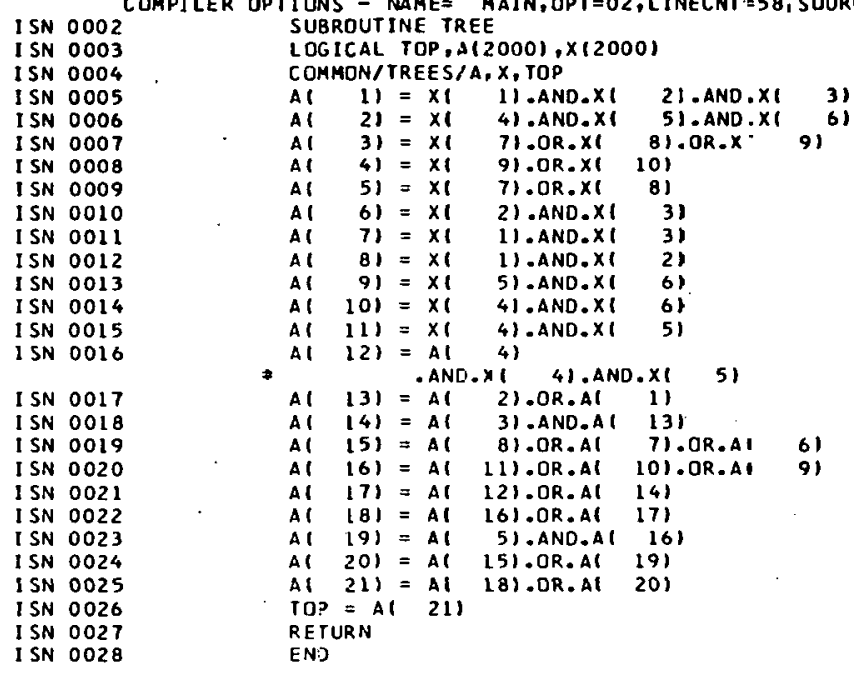

-OPt IONS IN EFFECT* NAME $=$ maIN, OPT $=02$, LINECNT=58

-OPTIONS IN EFFECT SUURCE, EBCOIC, NOLIST, NODECK, LOAD, NOMAP, NCEDIT, NOID, ROXPEF

*STATISTICS* SOURCE STATEMENTS = 27 . PROGRAM SIZE $=572$

*statistics* no diagnostics generated

****** END OF COMPILATION ****** 


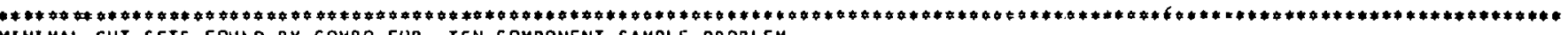
MENI MAL CUT SETS FOUND BY COMBO FILR TEN COMPONENT SAMPLE PRORLEM

MINT MAL CUT SET No

COMPS STI COMPG
CORRESPONDING GATE FAILURES-

$$
8 \text { 15 }
$$

1

MENIMAL CUT SET NO. ?

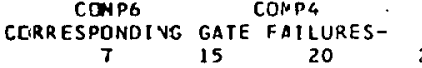

MINIMAL CUT SET NO. 3

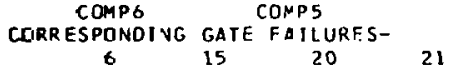

MINI MAL CUT SET NO. 4

COMP2
CORRESPONDING GATE FAILURES-
I1'

MINI MAL CUT SET NO. CONPI 5

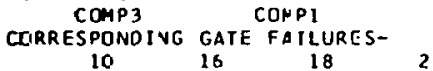

MINI MAL CUT SET ND.

COAP3
CORRESPONDING GATE FAILURES-

********* $16,18 \quad 21$ 


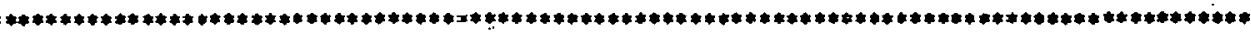
- TREBIL FAULT TREE BUILOING PROGRAR

TEN COMPONENT SAMPLE PROBLEM

NUMBER OF GATES,NG-

COMBO STARTING VALUE, MIN--

COMBO ending ValUe, MaX--

CUT SET - PATH SET SWITCH, IDEXI--

PRINT - PUNCH SHITCH, IDEX:2-

MONTE CARLO STARTER, MC S--

NO. OF RANOBM NUMBERS TO REJECT, NREJEC---- 100

NO. OF MONTE CARLO tRI ALS, NTR-D 1000

MIXING PARAMETER SWITCH, IREN-_-_ 1

MONTE CARLO MIXING PARAMETER, TAA--------..0 

MIN: MAL SETS FOR TEN CDMPONELT SAMP LE PROBLEM

$$
\begin{aligned}
& \text { MIN: YAL CUT SET } 1 \text { COMP1 } \\
& \text { SEI FAILURE PROBABILIIY }=5.12564 \text { E - } 03
\end{aligned}
$$

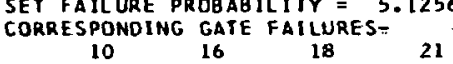

MIN: MAL CUI SET 2 TH TOMP TOL

SET FAILURE PROBABILITY $=3.99576 E-01$

$\underset{8}{\text { CORRESPONOING GATE FATLURES- }} 21$

MINI MAL CUT SET 3 THI: SET HAS FOUND ON TRIAL COMP4

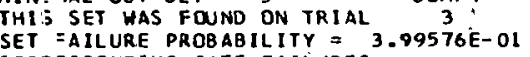

COMP3

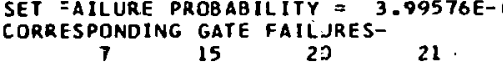

MINIMAL CUT SET 4 COMP

THIS SET WAS FOUND ON TRIAL 44
SET EAILURE PROBABILITY $=5.12564$ E-03

CORE ALURE PROBABILITY = 5.12564 E-0

COMP2

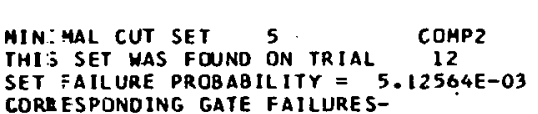

COMP3

$$
9{ }_{16}{ }_{1 B} \cdot 21
$$

MIN: MAL CUT SET

SET FAILURE PROBABILITY $=3.99576 E-01$

CORR :SPONOING GATE FAILURE S- 20

COMP5

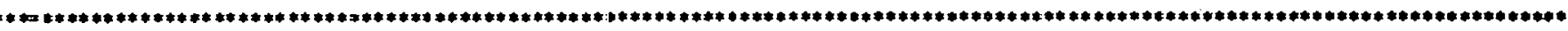

**** SUCh is fate ******

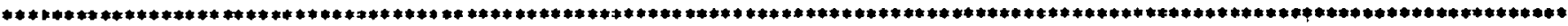




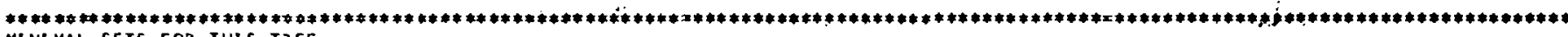
MINI MAL SETS FOR THIS TREE

等

$\begin{array}{lllll}\text { MINIMAL CUT SET } & 1 & \text { COMPI } & \text { COMP3 } \\ \text { MINIMAL CUT SET } & 2 & \text { COMP4 } & \text { COMPS } \\ \text { MINIMAL CUT SET } & 3 & \text { COMP4 } & \text { COMPG } \\ \text { MINIMAL CUT SET } & 4 & \text { COMP1 } & \text { COMP2 } \\ \text { MINIMAL CUT SET } & 5 & \text { COMP2 } & \text { COMP3 } \\ \text { MINIMAL CUT SET } & 6 & \text { COMP5 } & \text { COMP6 } \\ \text { *********} \text { END OF OUTPUT FROM MINSET *********}\end{array}$




\section{KITT-1 Run For The Sample. Tree}

Having obtained the minimal cut sets from PREP, the KITT codes were then run to obtain the probability characteristics associated with the sample fault tree. KITT-l was first run to illustrate the evaluation of a one phase problem. A listing of the input cards for the sample run is shown on page F14. The listing depicts the correct order and format for the input. All the components on the tree were assumed to have constant repair times of 100 hours. The number of components (Input Ǵroup 3), the component failure intensities and repair times (Input Groups 4 and 5), and the components in the minimal cut sets (Input Group 13), were punched out by PREP, in the proper format, and were merely inserted in the proper 1ocations in the KITT-I input. Because this output was used from PREP, the component indices for KITT-I are those assigned by PREP and are given in the cross-reference output of PREP (showing which index is assigned to which component).

For the sample run, the probability characteristics were obtained at 20 time points equally spaced at 20 hours, and the exact values of the system characteristics were obtained. The output from KITT-1 for the run is given on pages F15 to F29. 
SAMPLE PROBLEM FOR CONVERSION CHECKING

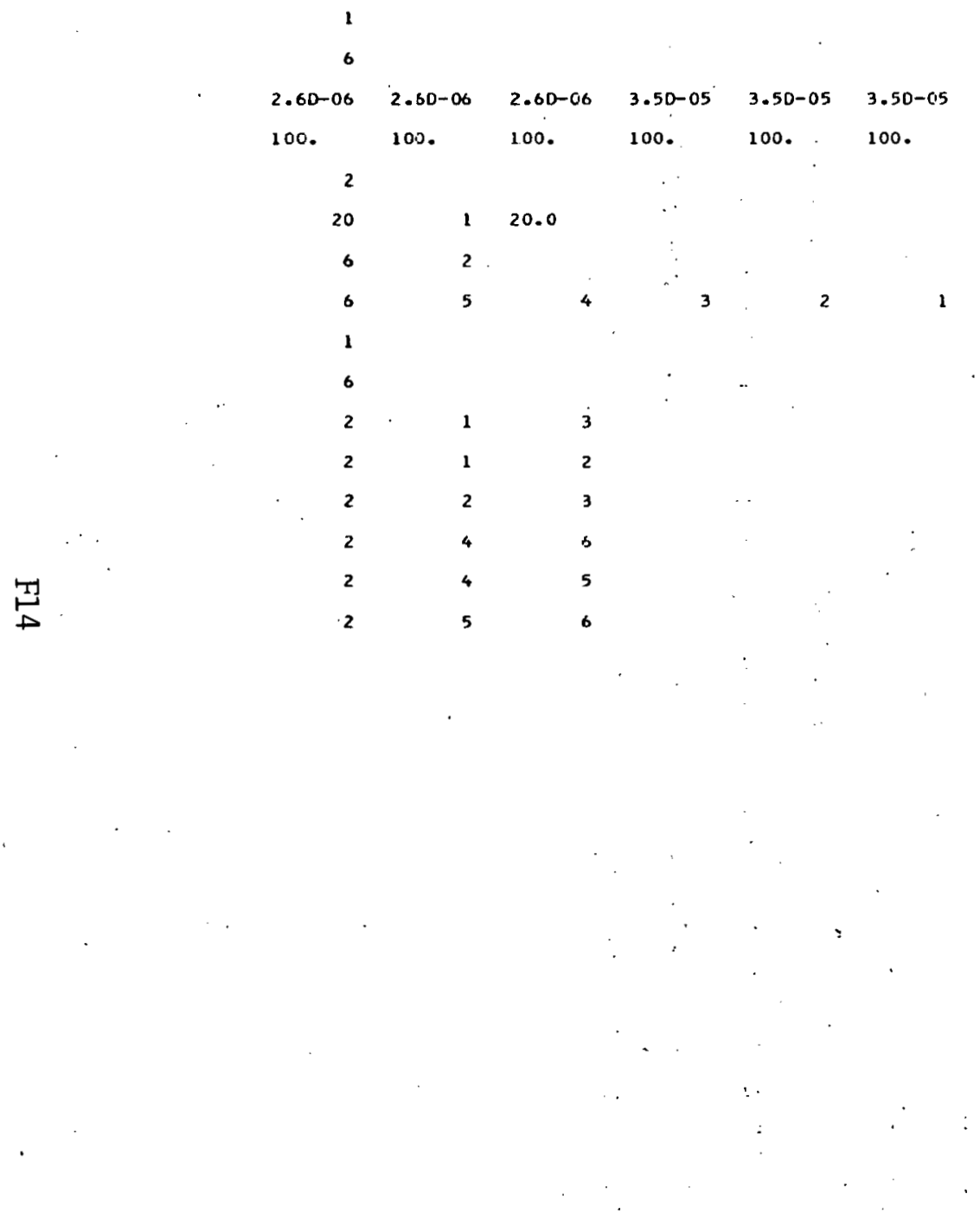




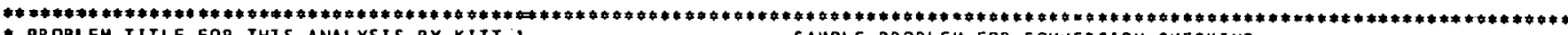

* PROBLEM TITLE FOR THIS ANALYSIS BY KITT-1. SAUPLF PROBLEM FOR - OYYFRSION CHFCKINT

No. OF PARAMETEF, RUNS (NPRCB) $=1$

NO. OF COMPONENIS AND INHIBTT CONDITIONS (NCOMP) $=10$

COMPONENT DATA ILAYBDA AND TAUI (NON-POSITIVE TAU OFNOTES NON-REPAIRI
INON-POSITIVE LAMRDA DENTTES INHIBTT CONDITIJNI

COMPONENT INDEX

COMPONENT INDEX
1
2
3
4
5
6
7
8
9
10

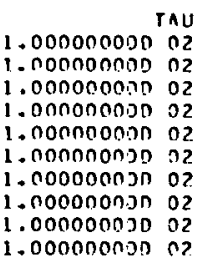

BRACKET FLAG IISTOPI. IF ISTOP=2 SYSTEM INFORMATION IS JBTAIVED FOBM RRAEKETING. IF ISTTP=1 IT IS NOT.

\section{NO. OF TIME POINTS (NTPI) $=20$}

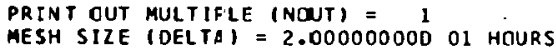

NUMBER OF OUTER BRACKETS (NBMAX) $=$ :
FATLURE RATE COFRECTION F(AAG (IFAGZ2) $=2$

INNER PRACKETS FOR IFAG? $=$ ?
OUTER ERACKET NO. (M), NO. OF INNER ERACKETS (NB2 (M))

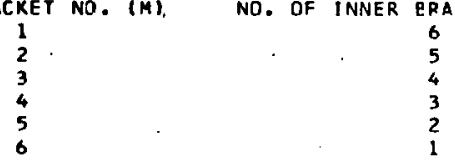

SET FLAG IPATHI. IF IPATH=1 MINIMAL CUT SETS ARE USFD. IF IPATH=? MINIMAL PATH STTS azF USEO.
FOR THIS PROBLEY IPATH = I

NO. OF SETS (NCUT) $=6$ 


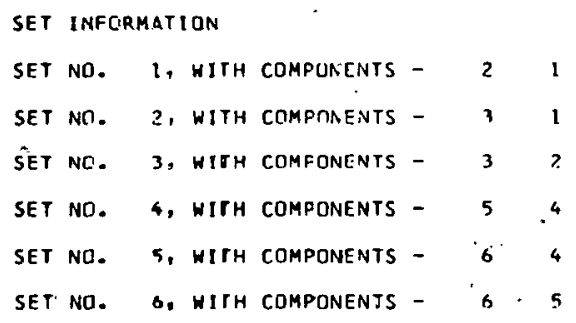

管

,

,

$\cdot$

; 
COMPONENT AND INHIBIT INFORMATION

CHARACTERISTICS FOR COMPONENT NO. = 1

$T$ (HOURS)

2.0000000001

8.00000000 01

1.0000000002

1.4000000002

1.6000000002

1.80000000 02

2.2000000002

2.4000000002

.6000000002

2.80000000
3.00000000

3.2000000002

3.6000000002

I (HOURS)

2.000000000

10

6.00000000 ol

1.0000000002

1.2000000002

1.4000000002

1.60000000
1.80000000
02

2.0000000002

2.20000000 0?

2.4000000002

2.6000000002

3.0000000002

3.2000000002

3.4000000002

3.6000000002

T I HOURS

0.0

4.00000000 01

6.00000000 01

8.00000000 ol

1.00000000 02 $3.48778010-03 \quad 3.48779270-05 \quad 3.50000000-05$

CHARACTERISTICS FOR COMPONENT NO. =

0

0.0

$3.50000000-05$

$3.50000000-05$

$1.39877570-03$

$3.49755210-05$

$3.49265860-0$

$3.49021500-05$

$3.49205030-03$

$3.48777780-05$

3.50000000-05

$3.50000000-05$

$3.50000000-05$

$3.50000000-05$
0

0.0
$6.99387860-04$
$1.39877570-03$
$2.09755210-03$
$2.79571710-03$
$3.49388210-03$
$3.49205030-03$
$3.49021860-03$
$3.4889990-03$
$3.48839250-03$
$3.48778570-03$
$3.48778810-03$
$3.48779060-03$
$3.48779200-03$
$3.48779240-03$
$3.48779270-03$
$3.48779270-03$
$3.48779270-03$
$3.48779270-03$
$3.48779270-03$

$3.50000000-05$
$3.49755210-05$
$3.49510430-05$
$3.49265860-05$
$3.49021500-05$
$3.48777140-05$
$3.48777780-05$
$3.48778420-05$
$3.48778850-05$
$3.48779060-05$
$3.48779280-05$
$3.48779270-05$
$3.48779270-05$
$3.48779270-05$
$3.48779270-05$
$3.48779770-05$
$3.48779270-05$
$3.48779270-05$
$3.48779270-05$
$3.48779270-05$

$3.50000000-05$ 3.50000000-05 $3.50000000-05$ $3.50000000-05$ $3.50000000-05$ $3.5000000-05$ 3.50000000-05 $3.50000000-05$ $3.50000000-05$ $3.55000000-05$ $3.5000000-05$ $3.50000000-05$ $3.5 .0000000-0.5$ $3.50000000-05$ $3.50000000-05$

CHARACTERISTICS FOP TOMPONENT NO. =

0.0

0

$\begin{array}{lll}0.99387860-04 & 3.50000000-05 & \mathbf{3 . 5} 50000000-05\end{array}$ $\begin{array}{lll}2.09755210-03 & 3.49255860-05 & 3.50000000-05 \\ 2.79571710-03 & 3.49021500-05 & 3.50000000-05\end{array}$ $3.49388210-03 \cdot 3.4877714 D-05 \quad 3.50000000-05$ $3.49205030-03 \quad 3.48777780-05 \quad 3.50000000-05$ $3.48778420-05 \quad 3.50000000-05$

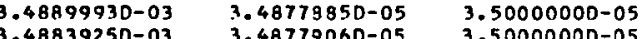
$3.48778570-03 \quad 3.48779280-05-3.50000000-05$ $3.48779060-03 \quad 3.48779270-05 \quad 3.50000000-05$ $3.48779200-03 \quad 3.48779270-05 \quad 3.50000000-05$ $3.48779270-03 \quad 3.48779370-05 \quad 3.50000000-05$ $3.48779270-03 \quad 3.48779270-05$ $\begin{array}{lll}3.48779270-03 & 3.48779270-05 & 3.50000000-05 \\ 3.48779270-03 & 3.48779270-05 & 3.50000000-05\end{array}$ $\begin{array}{lll}3.48779270-03 & 3.4877927 D-05 & 3.500 .00000-05 \\ 3.48779270-03 & 3.48779770-05 & 3.50000000-05\end{array}$

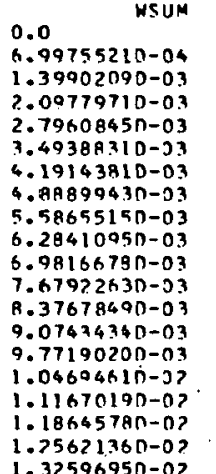

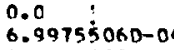
$1.39902050-03$ $7.79608370-03$ $3.49388210-03$ $4.19110230-03$ $5.58434920-03$ $6.29019660-03$ $6.97555710-03$ . 05a72030-0 $9.75213650-n 3$ $1.04450670-07$ i. $18294750-02$

$1.32119460-0$ ?

\begin{tabular}{|c|c|}
\hline \multicolumn{2}{|l|}{$0 . n \quad$ WSUM } \\
\hline $\begin{array}{l}0 . n \\
x .09755210-04\end{array}$ & \\
\hline & \\
\hline $2.09779710-03$ & $2.09779650-03$ \\
\hline $7960 R 45 n-03$ & $2.79608370-03$ \\
\hline $88310-33$ & \\
\hline $4.19143910-03$ & 4.1911923 \\
\hline $\begin{array}{l}B 8 B 95 \\
5865\end{array}$ & $\begin{array}{l}4.8 B B 146 D-03 \\
5.5843492 D-03\end{array}$ \\
\hline 0 & $6.28019660-03$ \\
\hline & 9755571 \\
\hline & $7.67043090-03$ \\
\hline & $8.36481860-03$ \\
\hline & 587 \\
\hline 3 & $0-03$ \\
\hline & 12 \\
\hline & \\
\hline & \\
\hline & \\
\hline 969 & \\
\hline
\end{tabular}

$0.0 \quad$ HSUM

0.0
$0.97755710-04$

$1.39907090-03$
$2.09779710-03$

$3.79608450-0.3$

$3.4938310-03$
$4.1914381 \mathrm{D}-03$

0.0

F SUM

$0.09975506 n-04$

$.39902050-03$
$.09770650-03$

?. $79608370-03$

$3.49388210-03$
$4.19119230-03$ 


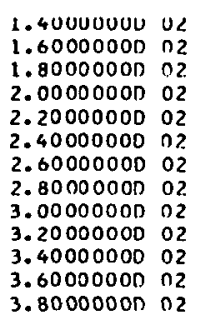

(HDURS

2.000000000 6.00000000 01

8.00000000 01 $\begin{array}{lll}1.00000000 & 02 \\ 1.20000000 & 02\end{array}$ $1.40000000 \quad 02$ 1.6000000002 1.8000000002 2.2000000002 2.40000000 02 .80000000 02 .00000000 D2 3.2000000002 3.60000000 02

$\begin{array}{lll}3.60000000 & 02 \\ 3.80000000 & 02\end{array}$

I (HOURS) 4.00000000 ol 6.00000000 ol . 1.2000000002 1.4000000002
.6000000002 .80000000 or 2.0000000002 2.4000000002 2.6000000002 2.8000000002 3.0000000002
3.20000000 3.40000000 3.6000000002 3.80000000
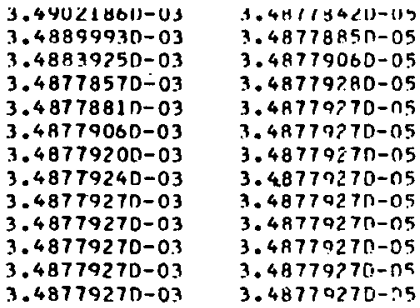

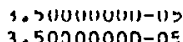
. $3.50000000-05$ $3.50000000-05$ $3.50000000-05$ $3.50000000-05$ $3.50500000-05$ $3.50000000-05$ $3.50000000-0$ $3.50000000-0$
$3.50000000-05$ $3.48770270-$ ?5

CHARACTERISTICS FOP SUMPONFNT NO. =

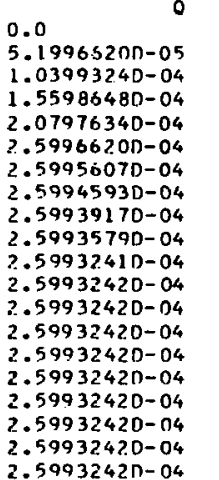

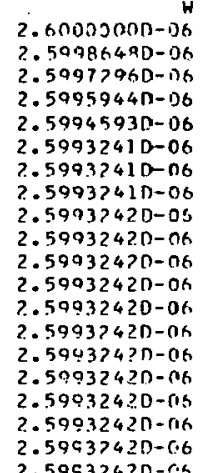

$2.60300 n 0 n-0$ $.60000 n n 0-05$
$.50000000-16$ $2.60000000-06$ $2.60000000-06$ $2.40000000-06$ -5
$.50005030-05$ 3.600000000 (2.60000000-06 $2.60000000-06$ $2.60000000-0$ $2.60000000-05$ $2.50000000-25$ $2.60000000-06$ -5
$.60000000-06$ $3.60000000-5$ $2.60000000-0.5$

CHARACTEOISTICS FORP. COMPNASENT NO. $=$

0.0
$5.19966200-05$
$1.03993240-04$

$1.03993240-04$
$1.55986480-04$

$2.0797634 \mathrm{D}-04$

$2.5 .9956070-04$

$2.59945930-04$

$2.59939170-04$

$2.59935790-04$

$2.59932420-04$

$2.59932420-04$
$2.59932420-04$

$2.59932420-04$

$2.599324200-04$

$5.59932420-04$

$2.59932420-04$

$2.60000000-\mathrm{CH}$

$2.59972900-06$

$2.59959440-06$

$2.59932410-06$

$2.59032410-06$

2.59.932410-O6

$2.59732420-06$

$2.59932470-06$
$2.599324<0-06$

$2.59932420-05$

$2.59932420-n 5$

$2.59932420-0 \mathrm{H}$

$2.59932420-05$

$2.599324200-05$

$2.59932420-0.5$

$2.59932 .42 n-n 6$
$2.5993242 \pi-08$

$2.60000000-05$ $2.60000000-06$ $2.60000000-0.5$
$2.60000000-05$ $2.60002000-0$

6500030nn-n6

(1)

. $500000000-06$

$2.60000000-06$

$2.60003000-06$

$2.60000000-0 h$

$2.50003000-06$

$2.60000000-06$

$2.60703000-06$
$2.50000000-06$
2.600000006

.60000000 -06

$2.60000000-05$
$2.60000000-06$

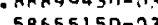
(1)

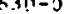
..3767840n-n $0.0776462-03$ 04674410-32

$.25621350-07$
$.37506050-07$

4. $5 \times 3014011-(1)$

$6.78019660-05$

$6.97555710-03$

7.5704309003

R. 364A1860-n?

$0.75213650-03$

1. n445067n-n?

$1.11375140-0$ ?

-1
0

1. $2 ? 11946 n-n$ ?

WS:IM

$5.19986490-35$

1. $5599783 n-04$

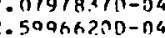

$.11052890-94$

$415025 \times 5 n-94$

$1980 R 62 n-n 4$
4

.7585807n-04

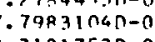

8. $3191752 n-04$

Q. $35790400-04$

Q. $9777507 ก-n$

5.19388480-05

$1.55287830-04$

$2.07978370-04$

2. 59965200-04

$3.11051330-04$

$4.1591348 n-04$

$4.67890510-04$

$5.1085482 n-n 4$

S.7183644B-त4

$0.75771560-04$

$7.37735070-040$

9. $3165 \times 990-n 4$ A. $93609300-04$ $0.8751 ? 000$ - 04

$5.1098640 n-65$

$.0390459 n-04$

2. $0797837 n-04$

$599.562300-94$

$.11952 \times 90-04$
$.63930170-04$

$.15025 \times 5 \pi-04$

$4.67912130-04$

$6.238715 .90-04$
$6.7585807 n-04$

7. $784455 n-04$

?.79831040-04
8. $31817920-04$

a $3304 n 00-04$

$.35700490-04$
$.8777607 n-04$

$5.07998480-75$ $1.5947830-94$

$.50966200-174$

$7.11951330-04$

$3.63933760-04$

$4.15913480-144$

$5.19864320-04$

$5.71836440-04$

6. $23805350-04$

$7.27735 n 10-04$

8. $31653080-04$

$9.87512090-04$ 
T (HOURS) 2.00000000 01 4.00000000
6.00000000 6.00000000
8.00000000 1.0000000002 1.2000000002 1.4000000002
1.6000000002 1.6000000002
1.8000000002 2,0000000002 2.2000000002 2.4000000002 2.6000000002 2.8000000002 3.2000000002 3.4000000002 3.60000000
3.80000000
T (HOURS)

2.00000000 01 6.00000000 ol 8.00000000 01 1.0000000002 1.40000002 1.6000000002 1.8000000002 2.0000000002 2.2000000002 2.600000002 2.80000000 02

3.0000000002

3.2000000002 3.6000000002 3.8000000002

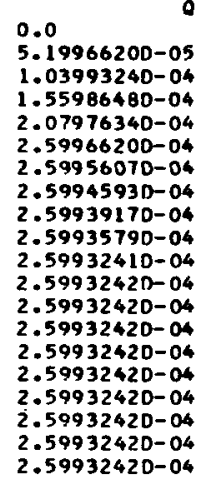

$2.60000000-06$
$2.59985480-06$
$2.59972960-06$
$2.59959440-06$
$2.59945930-06$
$2.59932410-06$
$2.59932410-06$
$2.59932410-06$
$2.59932420-06$
$2.59932420-06$
$2.59932420-66$
$2.59932420-06$
$2.59932420-66$
$2.59932420-06$
$2.59932420-66$
$2.59932420-06$
$2.59932420-06$
$2.59932420-06$
$2.59932420-06$
$2.59932420-06$

$2.60000000-06$
$2.60000000-06$ $2.60000000-0$ $2.60000000-06$ . $2.60000000-06$ $2.60000000-06$ $2.60000000-06$ $2.60000000-06$ $2.60000000-06$ $2.60000000-06$ $2.60000000-06$ $2.60000000-06$
$2.60000000-06$ 0.0
$5.19986480-05$ $1.03994590-04$ $1.55987830-04$ 2.07978.370-04 $3.11952690-04$ $3.63939170-04$ $4.15925650-04$ $5.19898620-04$ $5.71885100-04$ $6.23871580-0^{4}$ 6.75858010-04 $7.27844550-04$ $8.31817520-04$ $8.83804000-04$ $9.35790490-06$ $9.87776970-04$

\section{0 $.19986480-05$ $.03994590-04$ $2.07978370-04$ $599662.00-04$
$.11951330-04$ $3.63933760-04$ $4.15913480-04$ $.19864820-04$ $6.23805350-04$ $6.75771560-04$ $7.79695880-04$
$8.31653980-04$} $9.3556209 \mathrm{D}-04$

CHARACTERISTICS FOR COMPONENT NO, $=7$

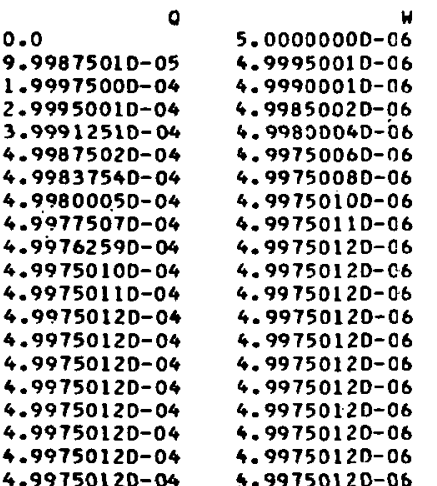

5.00000000-06 $5.00000000-06$ $5.00000000-06$ $5.0000000 \mathrm{D}-06$ $5.00000000-06$ $5.00000000-0$ $5.00000000-06$ $5.00000000-06$ $5.00000000-06$ $5.0000000-06$ $5.00000000-06$ $5.00000000-06$ $5.00000000-06$ $5.00000000-06$

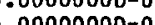
$5.00000000-06$ $1.09980000-04$ $4.99875020-04$ $5.9925040-04$ $7.99725080-04$ R. $99675130-96$ $9.99625120-04$ $1.09957510-03$ $1.29947520-03$ 1. $39942520-0.3$ $1.49937520-0$ ? $1.59932530-03$ $1.79922530-03$ $1.79922530-03$ $2.99955010-04$
$3.09920010-04$
FSUM 0.0
$9.99950000-05$ $.99980000-04$
$.99955000-04$ $3.99920010-04$ $4.99875020-04$ (. $7.99680090-04$ 9.99595120-04 $9.99500170-04$ $1.09939520-03$ $1.19928030-03$ $1.39902050-03$ $1.49887560-0.3$ $.59872070-03$
. (.698580 1.89819610-03 
CHARACTERISTICS FOR COMPONENT NO. $=10$

T (HOURS) 2.00000000 01 4.000000000 6.00000000 o 1.0000000002 1.2000000002 1.4000000002 1.3000000
1.02 2.00000000 02 2.2000000002 2.4000000002 2.60000000
2.80 2.800000002 3.2000000002 3.40000000 02 3.60000000 of
3.50000000 ?
$8.00000000-06,8.00000000-06$ $\begin{array}{ll}7.99872030-06 & 8.00000000-06 \\ 7.99744050-06 & 8.00000000-06\end{array}$ $\begin{array}{ll}7.99744050-06 & 8.00000000-06 \\ 7.99616100-06 & 8.00000000-06\end{array}$ $7.99616100-06$
$7.09488180-06$ $7.99350260-06 \quad 8.00000000-06$ $7.99360330-06 \quad 8.00000000-06$ $7.99360410-06 \quad 8.00000000-06$ 1.99360460-06 $\quad$..00000015-06 $7.9936051 \mathrm{D}-06$ Q $00000000-06$ 7.993605ID-06 * 8.0000000n-06 $7.9936051 \mathrm{D}-06$ B.00000000-06 $7.9936051 \mathrm{D}-06 \quad 8.00000000-06$ $7.99360510-06 \quad 8.00000000-05$ $7.99360510-06 \quad$ B. $00000000-06$ 7.9936051J-06 8.0000JnoD-06 $\begin{array}{rr}7.9936051 \mathrm{D}-06 & 8.00000000-06 \\ 7.9935051 \mathrm{D}-06 & \text { B. } 00000000-06\end{array}$

\begin{tabular}{|c|c|}
\hline 0.0 & FSUM \\
\hline & $10-04$ \\
\hline & \\
\hline & \\
\hline 397057 & 6.3979 \\
\hline $\begin{array}{l}7.996 R 0130-34 \\
0.5955216 n-04\end{array}$ & $\begin{array}{l}7.99680000-04 \\
9.59530300-04\end{array}$ \\
\hline $1.119+242 n-03$ & $1.11937300-03$ \\
\hline $063 n-03$ & $1.77918110-03$ \\
\hline $1.43916840-03$ & $1.43896370-03$ \\
\hline $1.5990405 n-03$ & $1.59872070-03$ \\
\hline $1.75891260-07$ & $1.75845210-03$ \\
\hline $1.9 \mid \beta>R 47 n-03$ & $1.91815800-03$ \\
\hline 2.0785659n-03 & $2.07783830-03$ \\
\hline $2.23852800-0.3$ & $2.23749310-03$ \\
\hline 2.3794 CIOn-03 & $2.3971>730-03$ \\
\hline $2.5582731[1-03$ & $2.55572600-03$ \\
\hline $2.7181452 n-02$ & $7163042 n-03$ \\
\hline $2.8780172 n-0.3$ & $7.87585690-03$ \\
\hline $3.04788040-07$ & $3.03538390-0.3$ \\
\hline
\end{tabular}


2.0000000001 4.00000000 01 8.00000000 01 .0000000002 .2000000002 1.4000000002 1.8000000 2.00900000 .02 2.2000000002 2.4000000002 2.60100000 02 3.0000000002 3.2000000002 $3.40000000 \quad 02$
3.6000000002 $\begin{array}{lll}3.60000000 & 02 \\ 3.80000000 & 02\end{array}$ 0.0
2.0000000001
4.00000000 $\begin{array}{ll}4.00000000 & 01 \\ 6.000000000 & 01\end{array}$ 8.00000000 01 1.00000000 ? 1.40000000022 1.6000000002 1.80000000 0? 2.0000000002 2.04000000002 2.6000000002 2.8000000002 3.0000000002 3.2000000002 3.6000000002
3.8000000002 0.0 1 0100000001 4.00000000 n 6.000000000
8.00000000 1.0000000002 1.20000000 ?
MINIMAL SET INFORMATION

ChARACTFRISTICS FIR SFT NO. =

0.0

0

$4.87143370-07$

$4.39972500-06$

$7.81603430-06$

$1.220721200-05$

$1.2194416 n-05$

$1.21816260-05$

$1.21688820-05$

$.21646490-05$

$1.21646660-05$

$1.22646830-05$

$1.21646930-05$

$1.21646980-05$

$1.21646980-05$

$1.21646980-05$

$1.21646980-05$
$1.21646980-05$

0.0

$.8922 \div 1,00-08$

$1.46520670-07$

$.95153030-n 7$
$.43717240-n 7$

$2.43589920-07$

$2.4346258 n-07$
$2.43377830-07$

$2.43335650-07$

$.4329334 \mathrm{TD}-07$
$2.43273 \times+0-07$

$2.4327332 \mathrm{D}-07$

$2.43293710-07$

$2.432 .73760-07$

.4329336n-07

$2.437 .9396, n-07$

0.0
$4.89229340-08$
$9.77775310-08$

$1.44521310-07$

$2.03726220-07$

$2.47592890-07$

$3.43385790-07$

$2.43338610-07$

$2.43295430-07$
$2.43795600-07$

$2.43295780-07$

$2.43205870-07$

$2.43296920-07$

$43296920-07$

$2.43296920-07$
$? .43296920-07$

$3.43296920-07$

CHARACTERISTIES FOR SFT NO. =

0

0.J
4.59143370-07

$4.39972500-06$

$7.31603430-06$
$1.22072120-05$

$1.22072120-05$

.

. 21731161$)-05$

$1.21688820-05$

$1.21646490-05$

$1.21646660-05$

$1.21646930-05$

$.21646960-05$

$1.22646980-05$

$1.21646980-05$

$1.21646987-05$

$1.21546980-05$

\section{0}

.89229.00-08

$.46520070-0$

$1.95153480-67$

$2.43717: 40-07$
2.4358902007

$2.43589 * 20-07$
$2.434620: 80-07$

$2.433778: 30-07$
$2.43325850-07$
2.43230

$2.43293 \times 10-07$
$2.43233<40-07$

$2.4323 .3<4 \mathrm{D}-07$
$2.4329382 \mathrm{D}-07$

$2.43293610-07$

$2.43293 \times 40-07$

$2.4329356 \mathrm{~J}-07$
$2.4329365 \mathrm{~S}-07$

(2.4329356

$2.4329366 i)-07$
$2.43293960-07$

\section{0}

$4.89229340-08$

$.46521310-07$
$1.05156600-07$

$1.05156500-07$

$2.43592390-07$

$7.43465550-07$

$2.43338510-07$

$2.43796430-07$
$2.43296600-07$

$2.43296780-07$

$2.432968770-07$

$2.43296900-07$
$2.43296970-07$

$2.43296970-07$

$2.4329692 \mathrm{n}-07$
$2.4329692 \mathrm{D}-07$

$2.43296920-07$

CharactFristics FOR SET NO. =

0.0
$4.89143370-07$
$1.95657350-06$

$1.95657350-06$
$4.39972500-06$

$1.22072120-05$

1. $21944160-05$
0.0

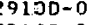

$1.46520670-07$

$2.437172 \div 0-07$

$2.43589920-07$
$1.45521310-07$

2.43720270-07

$2.43592890-07$
HSUM

$4.89229130-07$ $1.95623150-06$
$4.39921170-06$

$7.815949 \geq 0-06$

$1.22046520-05$

$1.7177340-09$

2.

3.16A37880-05

4.1415950n-05

$4.02818250-05$

$5.60135910-05$

$03724600-25$

$3300-05$

$7.0611218 n-05$
$7.5477097 n-05$

$7.54470970-05$
$8.07479770-05$

$4.89225220-07$ $.95673210-06$
$4.39921280-06$ $7.81595110-06$ 1.22046550-05 $1.70777150-05$ 2.194 A2040-05 $2.68165490-05$
$3.16836010-05$ $3.6549785 n-05$ $4.141552 .60-05$ $4.62812460-05$ $5.106940-05$ (6.0978?760-05 $7.06095140 \mathrm{D}=05$ $.54750870-05$
$.03406560-05$

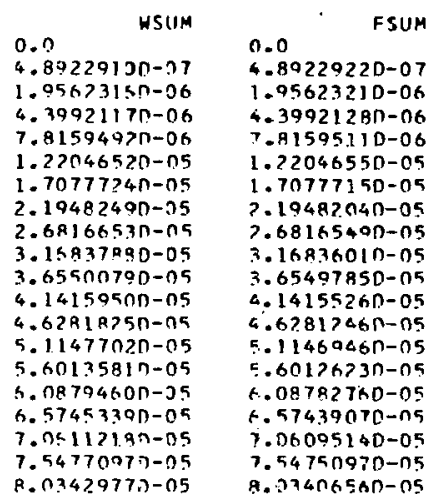
um

\section{\%}

$399212 B n-06$

. $22046550-05$

$.19482040-05$

$.16836010-05$

$.65497850-05$
$14155260-05$

0
0

$.6017623 n-05$
$087827 \times 0-05$

$8.034297720-05$ g. $034065 A 0-05$

$L$ NSUM

$0.0025300-06$
0.0

$1.95623160-06$

$4.39921170-06$
$7.81594920-06$

$1.22046520-05$

$1.70777740-05$

FSUM

$4.89279230-37$

$4.399 ? 1280-06$

7. B1595110-06
$1.22046550-05$

$1.70771150-05$ 


1.4000000002
1.6000000002
1.8000000002
2.0000000002
2.2000000002
2.4000000002
2.6000000002
2.8000000002
3.0000000002
3.2000000002
3.4000000002
3.6000000002
3.8000000002

T (HOURS)

0.0

2.000000000
4.00000000

$6.00000000 \cdot 01$

8.0000000001
1.0000000002

1.20000000 D2

1.40000000 0?

1.6000000002
1.8000000002

2.0000000002

2.4000000002

2.6000000002

3.0000000002

$\begin{array}{ll}3.60000000 & 02 \\ 3.80000000 & 02\end{array}$

1 (HOURS)

0.0000000001 $4.00000000 \cdot 01$

6.00000000
8.00000000

1.0000000002

1.20000000
1.40000000

1.4000000002

1.8000000002

2.0000000002

2.4000000002

2.6000000002
2.80000000
3.02

3.0000000002

3. 2000000002

$\begin{array}{lll}3.40000000 & 0 \text { ? } \\ 3.50000000 & 02\end{array}$

3.5000000002
3.80000000

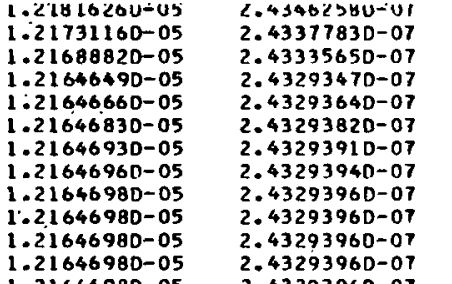
$2.43380790-07$ $2.4338640-07$ .43296600-07 $2.43296780-07$ (2.432.96870-07 (2.4.43296920-07 $2.43296920-07$
$2.43296920-07$ $2.43296920-07$ $2.43296920-07$

CHARACTERISTICS FOR SFI NO. $=$

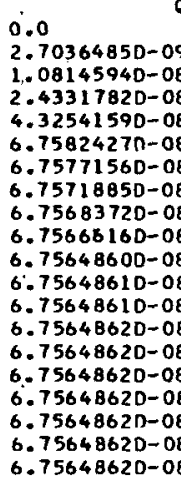

0.0

$n$ $2.70368370-10$
$5.40708610-10$ 8.11003180-10 $1.08125210-09$ $1.35142010-09$ $1.35136740-09$ $1.35133230-09$ $1.35131480-09$ $1.35129720-09$ $1.35129720-09$ $1.3512972 \mathrm{D}-09$ 1. $35129720-09$ 1. $35129720-09$ $1.35129720-09$ $1.35129720-09$ 0.0 $2.70368370-10$ .40708620-10 $1.0812521 n-0$ $1.35147290-09$ $1.35136750-0$ $1.35133240-09$ $1.35131490-09$ $1.35129730-09$ $1.35129730-09$ $1.35129730-09$ $1.35129730-09$ $.35129730-09$ $1.3512973 n-0$ $1.35129730-09$

CHARACTERISTICS FUP SET NO. 0.0
$2.70364850-09$ $1.08145940-08$
$2.43317820-08$ $1.43317820-08$
$4.32541590-08$
6.7592427000 $6.75824270-08$
$6.75771560-08$ $6.75718850-08$ $6.75683720-08$
$6.75666160-08$ $6.75648600-08$ $6.7564861 \mathrm{D}-08$ $6.756486110-08$ $6.75648620-08$ $6.7564862 \mathrm{D}-08$ $6.75648620-08$ $6.75648620-08$ $6.75648620-08$
$6.75648620-08$
$1014401440-0$, $2.6816653 \mathrm{n}-0 \mathrm{n}$ $3.65500700-05$ (1)

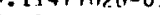
6. $.06112130-05$
$0.5470070-05$ $7.54770971-05$
$8.03420770-05$

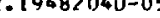
$2.68165490-05$ $3.65497850-05$ $4.14155260-05$ $4.62812460-0$ $5.11469460-05$ .601?h230-0 7.06095140-05 $7.54750970-05$
$8.03406960-05$ 0.0
$2.70368370-09$ $1.08144570-08$
$2.43315710-08$ $4.32541240-0 \mathrm{~A}$ $6.75813720-08$ $1.48665180-07$ $1.75691650-07$ $2.02717770-07$ ?.83795600-07 3.10B? 1540-07 3. $37847490-07$ (4.64 $4.45951 ? 70-07$ $2.70368370-09$ $2.43315710-08$ $1.21638180-07$ $.48665170-07$
$.75691640 \div 07$
$2.0717760-07$ $2.02717760-07$ $2.29763700-07$
$2.56769640-07$ $2.83795580-07$
$3.10821570-07$
$3.3767650-07$ $3.37847450-07$
$3.64873390-07$ $3.91899330-07$ $4.18925230-07$ $4.45051200-07$

2.70368270-09 $1.08144530-08$
$2.43315710-08$ $4.32541740-0$ B $6.75813720-0$ $? .46103020-0 \mathrm{~A}$ 1.2163818n-07 $1.75691650-07$ 2. $02717770-07$ $2.29743710-07$ 2.5674966n-07 3.10821540-07 3.37847400-0 $3.64873430-07$ $3.91899380-07$ $4.18075300-6$
$2.70368370-00$ $1.0814453 \mathrm{D}-08$
$2.43315710-08$ $4.3254124 n-08$ $6.7581373 D-D B$
$0.4610302 D-D A$ $1.21638180-07$ .75691640-07 ?.02717760-07 $.29743700-07$ . $83795580-07$ $3.37847450-07$
$3.64873390-07$ $3.91809330-07$ $4.45951200-07$ 
0.0

T (.HRUSR)

2.00000000 01

$\begin{array}{lll}4.00000000 & 21 \\ 6.00000000 & 01\end{array}$

8.0000000001

$1.00050 n 0002$

1.20000000 o?

1.60000000 n?

.800000002

2. 00000000 a?

2.2000000002

2.60000000 02

2.8000000002

.00000000 02

3. 20000000 0?

3.4000000002

3.60000000 0?

CHARACTERISTICS FOR SFT NG. =

\begin{tabular}{|c|c|c|c|c|}
\hline$\theta$ & W & $\mathrm{L}$ & נw. & Fsum \\
\hline & $n .0$ & 0.0 & 0.0 & 0.0 \\
\hline & $370-10$ & -10 & $n-$ & \\
\hline & $5.40708510-10$ & & $1.08144530-$ & $1.08144530-08$ \\
\hline $\begin{array}{l}2.43317820-08 \\
4.32541590-0 R\end{array}$ & $\begin{array}{l}8.11003180-10 \\
1.08125210-00\end{array}$ & $\begin{array}{l}0.11003200-10 \\
1.08125210-09\end{array}$ & $\begin{array}{l}2.43315710-02 \\
4.32541240-08\end{array}$ & $\begin{array}{l}2.43315710-08 \\
4.32541240-08\end{array}$ \\
\hline $6.75824270-08$ & $1.3514728 n-09$ & $1.35147290-09$ & $6.75813770-08$ & $6.7581373 \mathrm{D}-08$ \\
\hline & & & & 8 \\
\hline $75718.85 n-0 B$ & $1.35136740-09$ & $1.35136750-09$ & $1.2 .163 .8180-07$ & $1.2163818 n-07$ \\
\hline $37 ? n-08$ & $1.35133230-09$ & $.35133240-09$ & $1.48665180-07$ & $1.48665170-07$ \\
\hline $756661600-08$ & $1.35131480-09$ & $.35131490-09$ & $1.75691550-07$ & $1.75691640-07$ \\
\hline $48600-08$ & $1.35129720-00$ & $35129730-09$ & $71777 n$ & $2.0271776 n-07$ \\
\hline $49610-08$ & $1.35129720-09$ & $.35129730-09$ & $2.2974371 \mathrm{n}$ & $2.29743700-07$ \\
\hline $0-08$ & $1.35129720-09$ & $.3512 .7730-09$ & $.5676966 \mathrm{~N}-07$ & $7.56769640-07$ \\
\hline-03 & $1.35129720-09$ & $.35129730-09$ & 2.83795600 & $2.83795580-07$ \\
\hline 08 & 1.35129 & $73 n-09$ & $082154 \pi$ & $1520-07$ \\
\hline $75648620-08$ & $1.35129720-05$ & $.35129730-09$ & 37847490 & $37847450-07$ \\
\hline $.7564862 \mathrm{D}-08$ & $1.35129720-09$ & . $35129730-09$ & $487343 n-07$ & $3.64873390-07$ \\
\hline 75000 & $.35129720-0 c$ & $.35129730-09$ & $9189933 n-07$ & $.91899330-07$ \\
\hline & $1-09$ & & 7 & 17 \\
\hline $564862 n-08$ & $.35127720-09$ & $35129730-09$ & $.45951270-n 7$ & $.45951200-07$ \\
\hline
\end{tabular}


SYSTEM INFOPMATION-UPPER BOUNDS

DIFFERENTIAL CHARACTFRISTICS-UPPER BOUNDS

$T$ (HONRS)

0.0
2.0000000 .00001 4.0.030000000 01 . 1.00000000002 1.20000000002 1.40000030007 1.60000070002

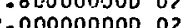
2.200000000 0) 2.400000000 0? 2.60000000002 . .20000000002 .4000030000 ? 3.600000000 n?
$3.80000 \cdot 2000$
$0 ?$

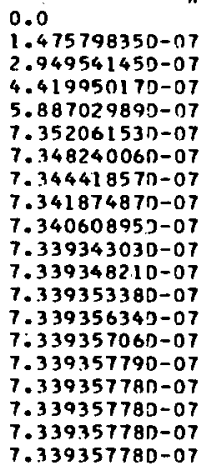

0.0

$0.07580053 n-07$
$2.049558860-07$

$4.420008830-07$
$5.897168600-07$

5. $187168600-07$

$7.348510380-07$

$7.344688470-07$

$7.342144480-07$

$7.340878420-07$

$7.339612360-07$
$7.339617540-07$

$7.339622720-07$

$7.339625670-07$

$7.339626400-07$

$7.339627120-07$

$7.139627120-07$

$7.339627120-07$

$7.339627120-07$

INTEGRAL SHARACTFRISTICS-UPPFR GOUNOS

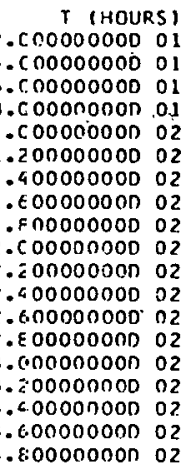

HSUN
$1.475798350-06$
$5.901138150-06$
$1.327067980-05$
$2.357760980-05$
$3.681670130-05$
$5.151700280-05$
$6.620966150-05$
$8.089595490-05$
$9.557843870-05$
$1.102583910-04$
$1.249370820-04$
$1.396157840-04$
$1.542944930-04$
$1.689732070-04$
$1.836519210-04$
$1.983306370-04$
$2.130093530-04$
$2.276880680-04$
$2.423667840-04$

FSUM
$.475799440-06$

$.901142510-06$

$2.357762720-05$

$3.681672840-05$

$5.151692170-05$

$3.620925570-05$

$8.089500840-05$
$0.557677570-05$

$.102557160-04$

$1.249332190-04$

$1.396105180-04$

$1.542876090-04$

. R.36411540-04

$1.983176050-04$

$2.129938400-04$

$2.276698600-04$ 
SYSTEM INFORMATICN-BEST BRACKETS

DIFFERENTIAL CHARACTERISTICS-BEST bRACKETS

\begin{tabular}{|c|c|c|c|c|c|c|c|}
\hline T I HOUR & RSI & OMIN & OAaX & MMIN & WMax & LMIN & $\operatorname{Lax}$ \\
\hline 0.0 & & 0.0 & 0.0 & 0.0 & 0.0 & 0.0 & 0.0 \\
\hline 2.00000000 & 01 & $1.47485660-06$ & $1.47485660-66$ & $1.47477740-07$ & $1.4747714 .0-07$ & $1.474773405-07$ & $1.47477360-07$ \\
\hline 4. 00000000 & 01 & $5.89668820-06$ & $5.896688820-06$ & $2.94543550-07$ & $2.9454365 n-07$ & $2.0454530 r:-07$ & $2.04545390-07$ \\
\hline 6.00000000 & 01 & $1.32537040-05$ & $1.32537040-05$ & $4.41072570-07$ & $4.41072570-07$ & $4.4107847 n-n 7$ & $4.4107 A 420-07$ \\
\hline 8.00000000 & 01 & $2.35341420-05$ & $2.3534142 D-05$ & $5.870653800-07$ & $5.87 n 65380-07$ & $5.8707920 n-07$ & $5.87079200-07$ \\
\hline 1.00000000 & 02 & $3.67390410-05$ & $3.67390410-.05$ & $7.32650 \geq 5[:-07$ & $7.32650250-07$ & $7.3267716 n-07$ & 7. $32677160-07$ \\
\hline 1.20000000 & 02 & $3.67007690-05$ & $3.67007690-05$ & $7.32270870-07$ & $7.32270770-07$ & $7.32297650-07$ & $7.32297650-07$ \\
\hline 1.40000000 & 02 & $3.66625170-05$ & $3.66625170-05$ & $7.31891300-07$ & $7.31891300-07$ & $7.31918130-07$ & $7.31918130-07$ \\
\hline 1.60000000 & 02 & $3.66370860-05$ & $3.66370660-05$ & $7.31639 \cdot 00-07$ & $7.31638707-07$ & $7.31655510-07$ & $7.31665510-07$ \\
\hline 1.80000000 & 02 & $3.6 .5264 .030-05$ & $3.66244030-05$ & $7.31513 n 00-07$ & $7.31513000-07$ & $7.91539790-07$ & $7.31939990-07$ \\
\hline 2.00000000 & 02 & $3.65117430-05$ & $3.66117430-05$ & $7.31387: 90-07$ & $7.31387290-07$ & $7.3141407 n-07$ & $7.31414070-07$ \\
\hline 2.20000000 & 02 & $3.666117950-05$ & $3.66117950-05$ & $7.313878 .00-07$ & $7.31387800-07$ & $7.31414580-07$ & $7.31414580-07$ \\
\hline 2.40000000 & 02 & $3.66118470-05$ & $3.66118470-05$ & $7.31388 \equiv 20-07$ & $7.31388320-07$ & $7.31415 .090-07$ & $7.31415090-07$ \\
\hline $2.600000-30$ & 02 & $3.66118760-05$ & $3.66118760-05$ & $7.31388<10-07$ & $7.31388615-07$ & $7.314: 53901-07$ & $7.31419390-07$ \\
\hline 2.80000000 & 02 & $3.56118830-05$ & $3.66118830-95$ & $7.31 .388<80-07$ & $7.313886800-07$ & $7.316: 5460-0.7$ & $7.31415460-07$ \\
\hline 3.00000000 & 02 & $3.66118910-05$ & $3.6611891)-35$ & $7.313 \times 9750-07$ & $7.31388750-07$ & $7.31415570-07$ & $7.31415570-07$ \\
\hline 3.20000000 & 02 & $3.66118910-05$ & $3.66118910-35$ & $7.31388750-07$ & $7.31388757-07$ & $7 . ? 141553 n-37$ & $7.31415530-07$ \\
\hline 00000 & 02 & $3.66118910-05$ & $3.66118910-35$ & $7.31388750-07$ & 7.31388 & $7.31+15530-07$ & $7.31415530-07$ \\
\hline 0000 & 0 & 3.6 & 3. & 7 & דיר & $7.71415530-07$ & $7.31419530-07$ \\
\hline 1000000 & 02 & $3.66118910-05$ & $3.6611 .8910-35$ & $7.31388750-07$ & $7.31388757-07$ & $7.3141953 n-07$ & $7.31415530-07$ \\
\hline
\end{tabular}


FAILURE RATE CONTRJBUTIONS

\begin{tabular}{|c|c|c|c|c|c|c|c|}
\hline I I HOUR & & WIMIN & W1 HAX & W2MIN & $\operatorname{W2MAX}$ & HMTN & HMAX \\
\hline 0.0 & & 0.0 & 0.0 & $0 . c$ & 0.0 & $0 . n$ & 0.0 \\
\hline 2.00000003 & 01 & $1.47528490-07$ & $1.47528490-07$ & $5.13476000-11$ & $5.13476000-11$ & $1.47477140-07$ & $1.47477140-07$ \\
\hline .00000000 & 01 & $2.94748910-07$ & $2.94748910-07$ & $2.05256210-10$ & $2.05256210-10$ & $2.04543650-07$ & $2.94543650-07$ \\
\hline .00000000 & 01 & $4.41533830-07$ & $4.4153383 D-07$ & $4.61255950-10$ & $4.61255950-10$ & $4.41072570-07$ & $4.41977570-07$ \\
\hline .00000000 & 01 & $5.37884260-07$ & $5.8788426 n-07$ & $8.18878170-10$ & $8.18878170-10$ & $5.97065380-07$ & $5.87065398 D-07$ \\
\hline 1.00000000 & $0 \tilde{z}$ & $7.33928350-07$ & $7.33928350-07$ & $1.27810180-09$ & $1.27810180-39$ & $7.32650250-07$ & $7.32650250-07$ \\
\hline .20000000 & 02 & $7.33547540-07$ & $7.33547540-07$ & $1.27676490-09$ & $1.27676493-39$ & $7.32270770-07$ & $7.32270770-07$ \\
\hline .40000000 & 02 & $7.33166720-07$ & $7.3316672 n-07$ & $1.27542870-09$ & $1.27542870-09$ & $7.31891300-07$ & $7.31991300-07$ \\
\hline .60000000 & $0 z$ & $7.32913240-07$ & $7.32913240-07$ & $1.27453960-09$ & $1.27453960-09$ & $7.3153870 n-07$ & $7.31638700-07$ \\
\hline 1.80000000 & 021 & $7.32787090-07$ & $7.32787090-07$ & $1.27409730-09$ & $1.27409730-09$ & $7.31513000-07$ & $7.31513000-07$ \\
\hline 2.00000000 & 02 & $7.32660940-07$ & $7.32660940-07$ & $1.27365500-09$ & $1.27365500-09$ & $7.31387290-07$ & $7.31387290-07$ \\
\hline 2.20000000 & 02 & $7.32661460-07$ & $7.32661460-07$ & $1.27365690-09$ & $1.27 .365690-09$ & $7.31387800-07$ & $7.31387800-07$ \\
\hline .40000000 & 02 & $7.32661970-07$ & $7.32661970-07$ & $1.27365870-00$ & $1.27365870-09$ & $7.3138 \times 320-07$ & $7.31388320-07$ \\
\hline .60000000 & 02 & $7.32662270-07$ & $7.3266227 n-07$ & $1.27365970-09$ & $1.27365970-09$ & $7.31388610-07$ & $7.31388610-07$ \\
\hline .80000000 & 02 & $7.32 .662340-07$ & $7.32662340-07$ & $1.27365990-09$ & $1.27365990-09$ & $7.3138868 n-07$ & $7.313886880-07$ \\
\hline .00000000 & 02 & $7.32662410-07$ & $7.32662410-07$ & $1.273660270-09$ & $1.27366020-09$ & $7.3138 A 750-07$ & $7.31388750-07$ \\
\hline .20000000 & 02 & $7.32662410-0$ & $7.32662410-07$ & $1.27366020-09$ & $1.27366 .020-09$ & $7.31388750-07$ & $7.31388750-07$ \\
\hline .4000 .0000 & 02 & $7.32662410-07$ & $.7 .32662410-07$ & $1.27386020-09$ & $1.27366020-09$ & $7.31383750-07$ & $7.31338750-07$ \\
\hline .6000 & 02 & $7.32662410-07$ & $7.32662410-07$ & $1.27366020-09$ & $1.27366020-09$ & $7.31388750-07$ & $7.313 \times 88750-07$ \\
\hline (5) & 02 & 7.32662410 & $32662410-07$ & $.27366020-09$ & $2736602 n-09$ & $.31383750-07$ & 7.3138875 \\
\hline
\end{tabular}


DIFFERENTIAL CHARACTERISTICS-LaST BRACKETS

T (HOURS)
0.0
2.0000000001
4.0000000001
6.0000000001
8.0000000001
1.0000300002
1.2000000002
1.4000000002
1.6060000002
1.8000000002
2.0000000002
2.2000000002
2.4000000002
2.6000000002
2.8000000002
3.0000000002
3.2000000002
3.4000000002
3.6000000002
3.8000000002

\begin{tabular}{|c|c|c|c|}
\hline OLAST. & MILAST & HZMIN-LAST & WZMAX-LAST \\
\hline 0.0 & 0.0 & 0.0 & 0.0 \\
\hline $1.47485660-08$ & $1.47528490-07$ & $5.13476000-11$ & $5.13476000-11$ \\
\hline $5.89668820-06$ & $2.94748910-07$ & $2.05256210-10$ & $2.05256210-10$ \\
\hline $1.32537040-05$ & $4.4153333 .0-07$ & $4.61255959-10$ & $4.61255950-10$ \\
\hline $2.35341420-05$ & $5.8788426 .0-07$ & $8.18878170-10$ & $8.18878170-10$ \\
\hline $3.67390410-05$ & $7.33928350-07$ & $1.27810180-09$ & $1.27810180-09$ \\
\hline $3.670 n 7690-05$ & $7.33547540-07$ & $1.27676499-09$ & $1.27676490-09$ \\
\hline $3.66625170-05$ & $7.33166720-07$ & $1.27542 .970-09$ & $1.27542870-09$ \\
\hline $3.66370660-05$ & $7.32913240-0 ?$ & $1.27453963-0.7$ & $1.27453960-09$ \\
\hline $3.66244030-05$ & $7.32787090-07$ & $1.27409730-09$ & $1 .>7409730-09$ \\
\hline $3.661174 .30-05$ & $7.3266094 \mathrm{D}-07$ & $1.27365500-09$ & $1.27365500-09$ \\
\hline $3.66117950-05$ & $7.32661460-07$ & $1.27365890-09$ & $1.27365690-09$ \\
\hline $3.66118470-05$ & $7.32661970-07$ & $1.27365870-09$ & $1.27365870-09$ \\
\hline $3.6611876 \mathrm{D}-05$ & $7.32662273-07$ & $1.27365970-09$ & $1.27365970-09$ \\
\hline $3.86118830-05$ & $7.32662340-07$ & $1.27365990-09$ & $1.27365990-09$ \\
\hline $3.66118910-05$ & $7.32667 .410-07$ & $1.27366020-09$ & $1.27366020-09$ \\
\hline $3.66118910-05$ & $7.326624110-07$ & $1.27366020-09$ & $1.27366020-09$ \\
\hline $3.66118910-05$ & $7.32662410-07$ & $1.27366020-09$ & $1.27366020-0.9$ \\
\hline 3.661189 & 7.32662410 & 1.2736 & $1.27366020-09$ \\
\hline $3.66118910-05$ & $.7 .32662410-07$ & $1.27366023-09$ & $1.27366020-09$ \\
\hline
\end{tabular}


INTEGRAL CHARACTERISTICS-BEST RRACKETS

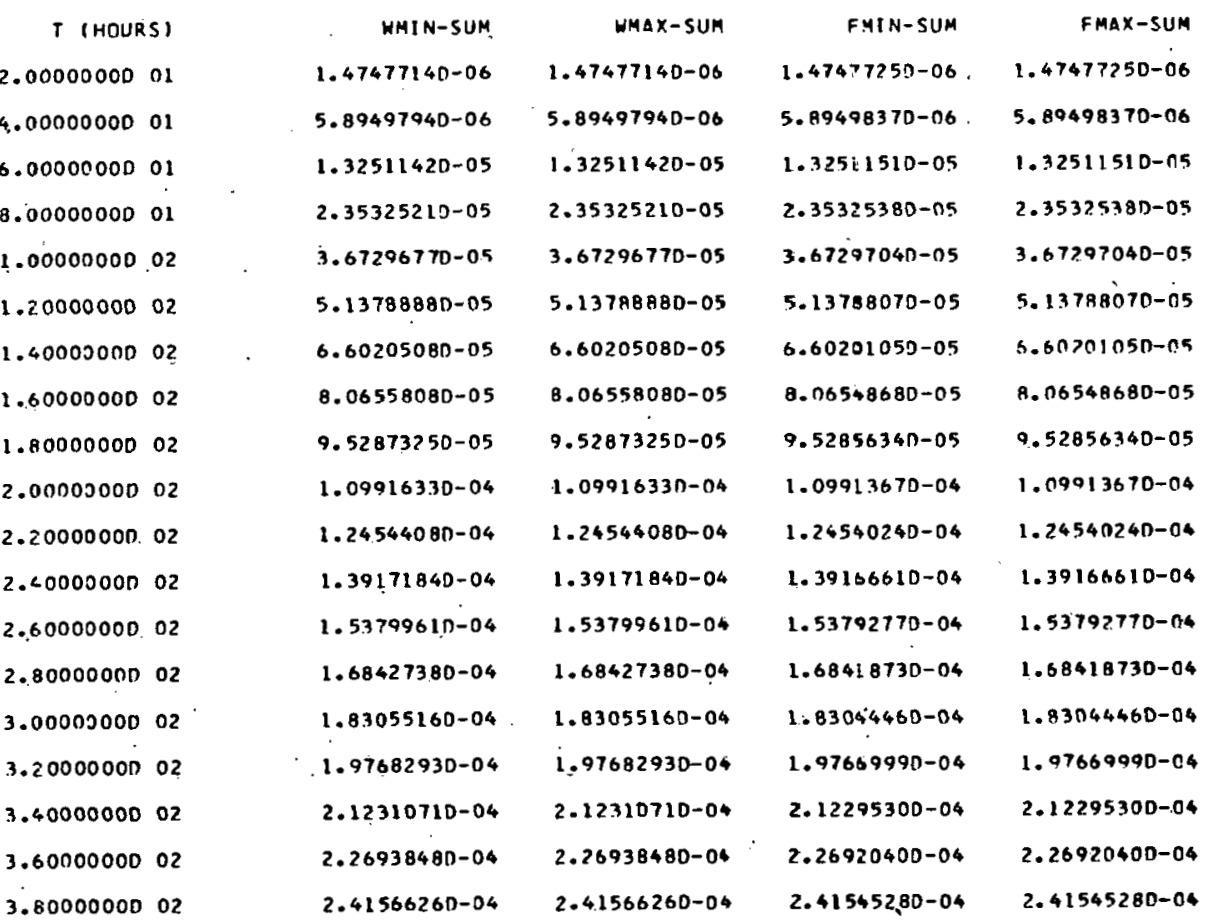

*****CONCLUSION OF OUTPUT FROM KITT-1***** 


\section{KITT-2 Run For The Sample Tree}

To illustrate the evaluation of a multiphase problem, KITT-2 was then run to determine the probability characteristics of the same fault tree. Pages F31 and F32 show a listing of the input cards to KITT-2 for this multiphase run. The minimal cut sets output by PREP were again used; the cards contuining the components in the minimal cut sets (Input Group 20) were those punched out by PREP and were merely inserted in the proper place for the KITT-2 run. The component failure intensities and repair times punched out by PREP could not be used for a multiphase run. The component indices are again those assigned by PREP and given in its crossreference output.

For the multiphase run, Components 3 and 6 had two phases with the first phase ending at 200 hours. In the first phase the two components were nonrepairable, while in the second phase they had constant repair times of 100 hours. The remaining components were one-phase arid had constant repair times of 100 hours. The same componont failure intersities were used as for the KITT-1 sample run. Since Components 7, 8, 9, and 10 were not in a minimal cut set, input data were not necessary for them.

As for the KITT-1 sample run, the probability characteristics were obtained at 20 time points equally spaced at 20 hours. The exact values for the system characteristics were again obtained. Pages F33 to F48 give the output from KITT-2 for this sample multiphase run. 


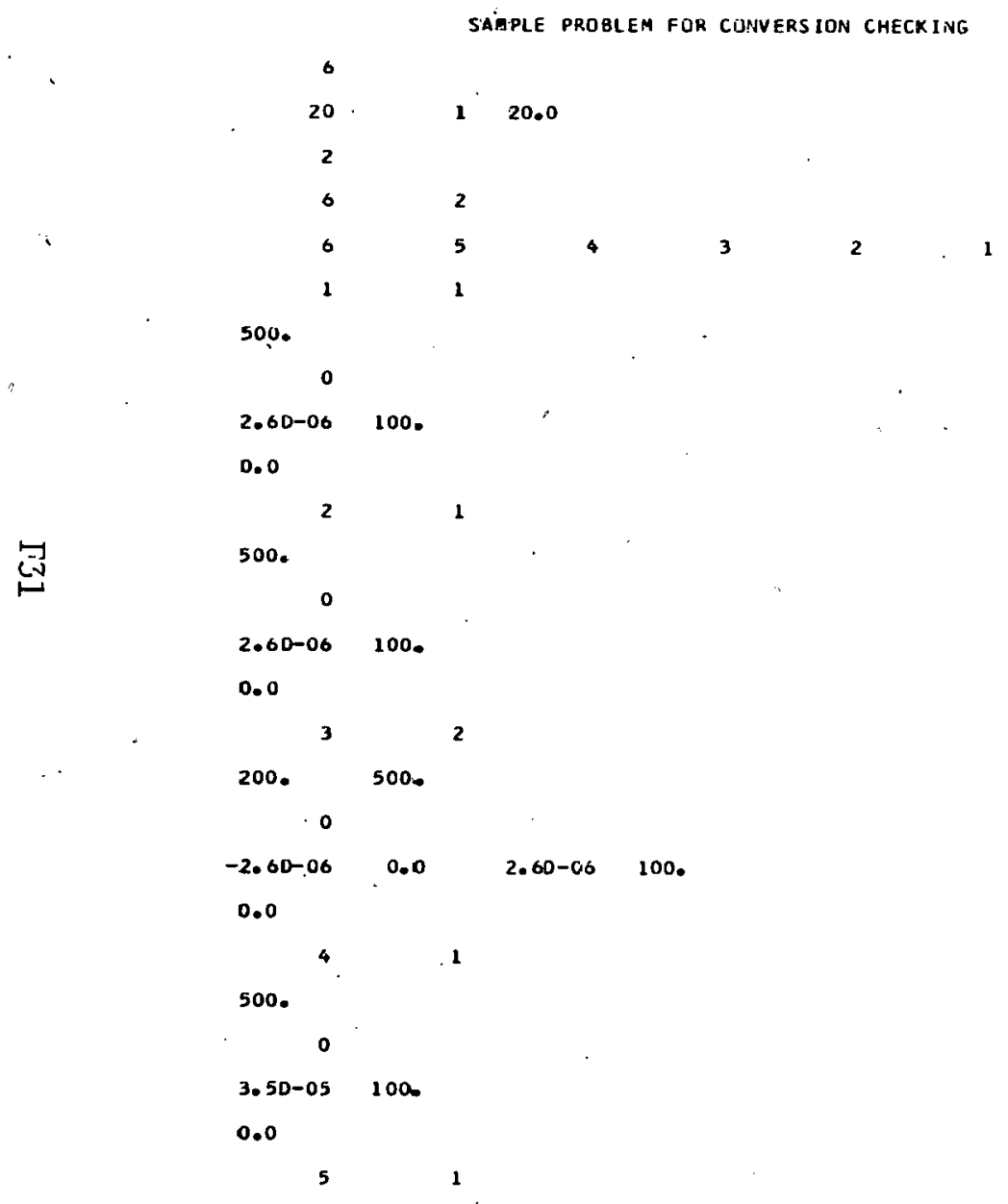


500.

3.50-05 100 .

0.0

500.

$\begin{array}{llll}-3.50-05 & 0.0 & 3.50-05 & 100 .\end{array}$

0.0

$\begin{array}{lll}1 & & \\ 6 & & 3 \\ 2 & 1 & 2 \\ 2 & 1 & 3 \\ 2 & 2 & 6 \\ 2 & 4 & 5 \\ 2 & 4 & 6 \\ 2 & 5 & \end{array}$




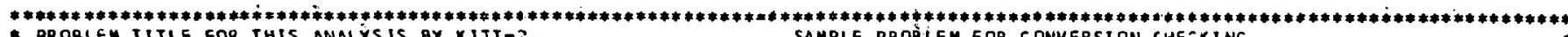
* PROBLEM IITLE FOR THIS ANALYS IS BY KITY-2,

NO. UF COMPONENTS SAND INHIBIT CONOITIONS INCOMPI $=" 6$

NOP OF TIME POINIS IN-PTI $=20$

PRINT OUT MULTIPLE N NOUTI $=1$ TOLLTA.I 2,000000000 OI HOURS

BRACKET FLAG (ISTOP.). IF ISTUP=2 SYSTEM INFORMATION IS OBTAINEO FROM BRACKET ING. IF ISTOP=1 IT IS NOT.
FOR THIS PRBLEA ISTOF $=2$.

NUMBER OF OUTER BRACKETS (NAMAX) $=0$
FAILURE RATE CORRECTION FLAG (IFAG 2 ) $=2$

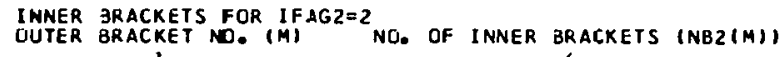

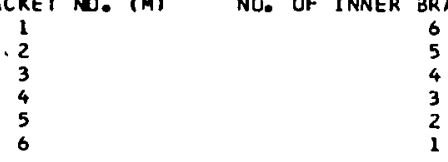


THERE ARE HO IMPOSEO BOUNDARY CONDITIONS (IBPHa $=0)$

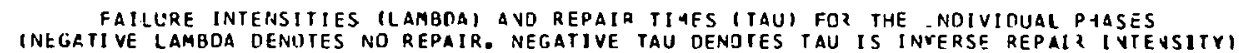
PMAS
LAMBOA
TAU
$1.000000000 \mathrm{CL}$
PHASE
LAMBDA

T IHOURSI

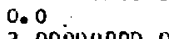

2.00000000 or
4.00000000 ol

$6.00000000 \cdot 0$

1.0000000002

10000000 .

$1.40000000 \mathrm{jz}$

$1.600000 C 002$

1.80000000 02

2.0000006002
2.2000006002

2.40600000 or

2.600 .0000002

2.80600000 02

3. 0000000 o

4000000002

3.6000000002
3.8000000002
GOMPONEVT RELIABILITY CHARACTEFISTICS

\begin{tabular}{|c|c|c|}
\hline $\begin{array}{l}0.0 \\
0.0 \\
5.199662000-05 \\
1.03993240-04 \\
1.55986430-04 \\
2.07976340-04 \\
2.55966200-04 \\
2.55956070-04 \\
2.59945930-04 \\
2.59939170-04 \\
2.59935790-04 \\
2.59932410-04 \\
2.59932420-04 \\
2.59932420-04 \\
2.59932420-04 \\
2.59932420-04 \\
2.59532420-04 \\
2.59932420-04 \\
2.59932420-04 \\
2.59932420-04 \\
2.59932420-04\end{array}$ & 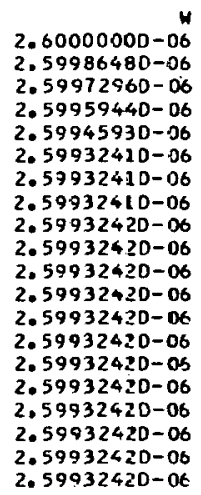 & $\begin{array}{l}2.60000000-06 \\
2.50000000-06 \\
2.60000003-06 \\
2.6000000)-06 \\
2.500000000-06 \\
2.50000000-06 \\
2.6000000)-06 \\
2.600000000-06 \\
2.600000000-06 \\
2.60000000-06 \\
2.600000000-06 \\
2.600000000-06 \\
2.60000000-06 \\
2.500000000-06 \\
2.500000000-06 \\
2.600000000-06 \\
2.660000000-05 \\
2.600000000-06 \\
2.600000000-06 \\
2.500000000-06\end{array}$ \\
\hline
\end{tabular}

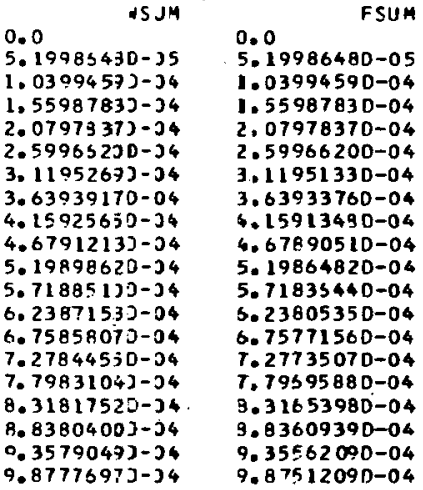


PHASE END TIMES CHOURSI
PHASE
¿.END TIME
PHASE
END TIME
PHASE
ENO TIME

THERE ARE NO IMPOSED BOUNDARY CONDITIONS (IBPHA=0)

FAILURE INTENSITIES (LAMBDA) AND REPAIR TIMES ITAUI FOR THE INDIVIDUAL PAASES
(NEGATIVE LAMBDA DENOTES NO REPAIR. NEGATIVE TAU DENOTES TAU IS INVERE REPAI? IVIEYSITY)

1.00000000002

PHASE

LAMBDA

TAU

INITIAL FAILED PROBAGILITY FDR PMASE 1 (000) $=0.0$

COMPONENT RELIABILITY CHARACTERISIICS

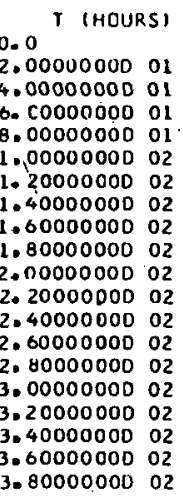

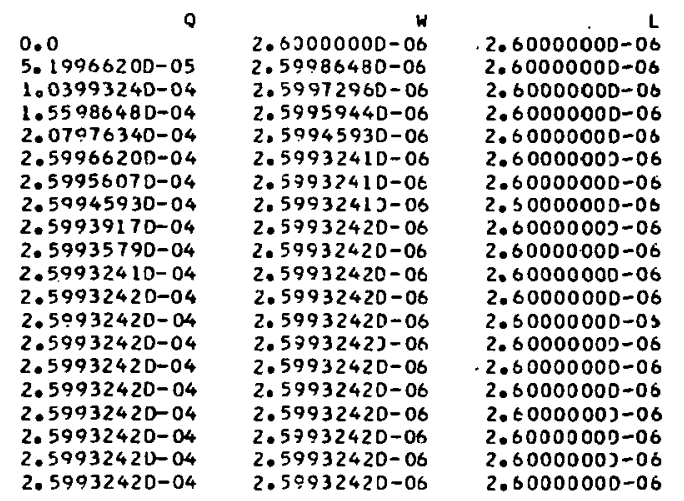

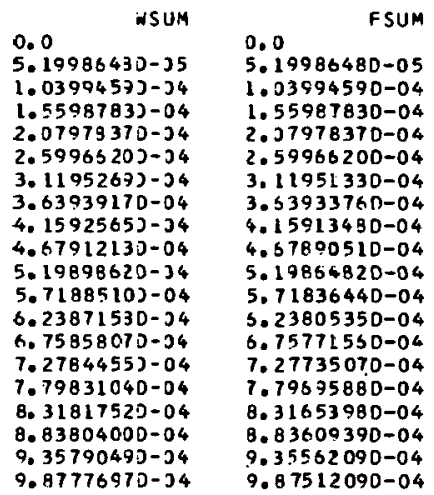


COMPONENT INOEX $=3$ NO. OF PHASES $=2$

PHISE ENO TIMES (HOURS)
PHASE
1 $\quad \begin{gathered}\text { ENO TIME } \\ 2.000000000\end{gathered}$

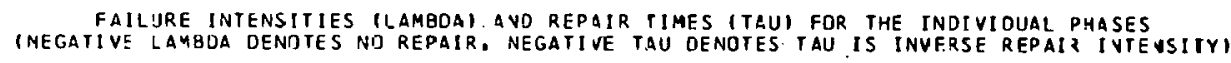
PHASE
LAMBDA
$-5000000000-0 B$
0.0 TAU
PHASE
LAMBDA
TAU
1.00000000002

INITIAL FAILED PROBAGILITY FOR PHASE 1 (000) $=0.0$

COMPONENT RELIABILITY CHARACTERISTICS

\begin{tabular}{|c|c|c|c|c|c|c|}
\hline & 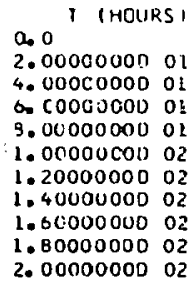 & $\begin{array}{l}0.0 \\
0.0 \\
5.19986480-05 \\
1.03994590-04 \\
1.53987830-04 \\
2.07978370-04 \\
2.59966200-04 \\
3.11951330-04 \\
3.63933760-04 \\
4.15913480-04 \\
4.67890510-04 \\
5.19864820-04\end{array}$ & $\begin{array}{l}W \\
2.50000000-00 \\
2.59986430-06 \\
2.59072980-06 \\
2.50959440-06 \\
2.59945930-06 \\
2.59932410-06 \\
2.59918293-06 \\
2.579053 E 0-06 \\
2.59891860-06 \\
2.59878350-06 \\
2.59864840-06\end{array}$ & $\begin{array}{l}2.600000000-06 \\
2.60000000-06 \\
2.60000000-06 \\
2.60000000-06 \\
2.60000000-06 \\
2.60000000-06 \\
2.60000000-06 \\
2.60000000-06 \\
2.60000000-06 \\
2.60000000-06 \\
2.60000000-06\end{array}$ & $\begin{array}{l}\text { NSJM } \\
0.0 \quad .0 \\
5.19986480-35 \\
1.0399457 J-34 \\
1.55987833-04 \\
2.07978370-34 \\
2.5996620 J-34 \\
3.11951330-34 \\
3.63933753-04 \\
4.15913490-04 \\
4.5789051 J-34 \\
5.1986482 J-04\end{array}$ & 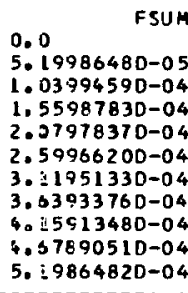 \\
\hline & 2.0000000002 & $\begin{array}{l}\text { RIGHT } \\
5.19864820-04\end{array}$ & $\begin{array}{l}\text { RCU VOAFY VLLUES } \\
2.59864840-06\end{array}$ & $\begin{array}{l}\text { FIDR PHASE } 1 \\
2.60300000-06\end{array}$ & $5=19864823-04$ & 5. $19864820-04$ \\
\hline & 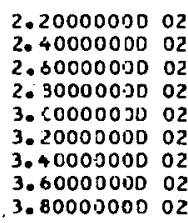 & $\begin{array}{l}5.71834410-04 \\
6.25804000-04 \\
6.7577021004 \\
7.27733050-04 \\
7.79695880-04 \\
2.5984750-04 \\
2.59864850-04 \\
2.59885130-04 \\
2.59908780-04\end{array}$ & $\begin{array}{l}2.59851320-06 \\
2.5983781[1-06 \\
2.50824300-06 \\
2.5981079[-06 \\
2.59797280-06 \\
2.59932446-06 \\
2.5993244[-06 \\
2.5993243[-06 \\
2.59932420-06\end{array}$ & $\begin{array}{l}2.60000000)-06 \\
2.60000000-06 \\
2.60000000-06 \\
2.60000000-06 \\
2.50000000-06 \\
2.60000000-06 \\
2.60000000-06 \\
2.60000000-06 \\
2.60000000-06\end{array}$ & $\begin{array}{l}5.7183844 J-04 \\
6.2380535)-04 \\
6.75771550-04 \\
7.27735073-04 \\
7.79695880-04 \\
9.31668850-04 \\
9.0365534 J-04 \\
9.35641830-04 \\
9.9762831 J-34\end{array}$ & $\begin{array}{l}5.11836440-04 \\
6.23805350-04 \\
6.75771560-04 \\
7.77735070-04 \\
7.79695880-04 \\
8.31653980-04 \\
8.33609390-04 \\
9.35562090-04 \\
9.07512090-04\end{array}$ \\
\hline
\end{tabular}

COMPONENT INDEX = 4 ND, OF PHASES = 


\begin{tabular}{|c|c|c|c|c|c|}
\hline PHASE & $\begin{array}{l}\text { END TIME } \\
5.000000000 \text { O2 }\end{array}$ & PHASE. & ENO TIME & RHASF. & END TIME \\
\hline
\end{tabular}

TMERE ARE NE IMPJSES BOUNRARY CONDITIONS (IGPHA=DI

FAILURE INTENSI ITES ILAMBOAI AND REPAIR TIMES ITAUI FOR THE INDIVIOUAL P PASES
INEGATIVE LAMBOA OENOTES NO REPAIR. NEGATIVE TAU OENOTES TAU IS INVERSE REPAI ? INTENSITYI
$+A$
1.00000000002
nuace
i A UOONA
$+\cdots$

IMITIAL FAILED OROBABILITY FOR PHASE $I(000)=0.0$

COMPONENT RELIABILITY CHARACTERISTICS

\begin{tabular}{|c|c|c|c|c|c|}
\hline 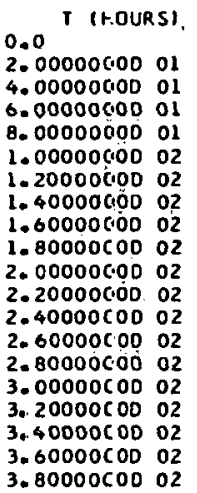 & $\begin{array}{l}0 \\
0.0 \\
6.99367860-04 \\
1.39877570-03 \\
2.09755210-03 \\
2.79571710-03 \\
3.49388210-03 \\
3.49265030-03 \\
3.49021860-03 \\
3.48859930-03 \\
3.488=9250-03 \\
3.487785700-03 \\
3.48778810-03 \\
3.48779060-03 \\
3.48779200-03 \\
3.48719240-03 \\
3.48779270-03 \\
3.487792700-03 \\
3.48779270-03 \\
3.48779270-03 \\
3.48779270-03\end{array}$ & 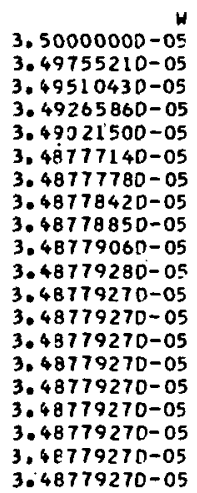 & 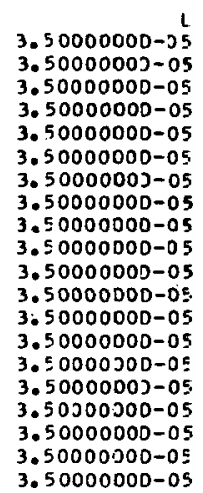 & 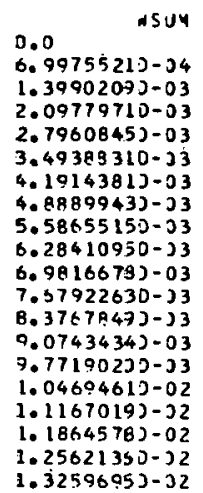 & 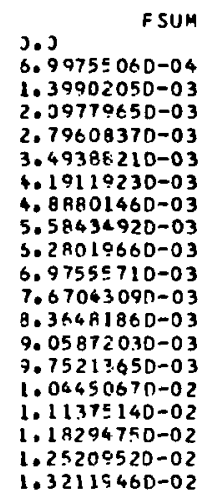 \\
\hline
\end{tabular}

COMPONENT IndEX $=5$ NO. OF Phases $=1$
PHASE $1, \quad$ END TIME
PHASE
END TIME.
DHASF.
ENO TIMF

PHASE END TIMES (HOURS) 


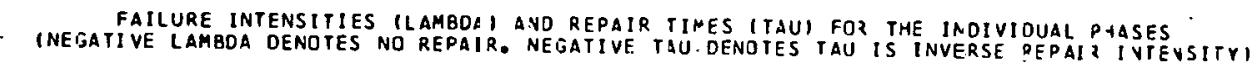
PHA:SE
LAMBDA
500000000-0
TaU
1.00000000002
PHASE
LAMBDA
$T A U$

INITIAL FAILEO PROBABILITY FOR PMASE $1(000)=0.0$

COMPUNEAI REL ?ABILITY CHÁRAC TERISTICS

1. IHOURS

$\begin{array}{lll}0.0 \\ 2.00000000 & 01\end{array}$

4. 000.90000 o

6.0000000001

8.000150000
1.0000000002

1. 2000000002

1.6000000002

1.80000000 .02

2. 0000000002

$2.20000000 \quad 02$

2.4000060002

2. 8000.000002

3.0000000002

3. 2000000002

3.40000000
3.60000000
3.80000000

$0.0 \quad 0$
$6.9938786)-04$
$1.39877570-03$
$2.09755210-03$
$2.79571710-03$
$3.45388210-03$
$3.49205030-03$
$3.49021860-03$
$3.48899930-03$
$3.48839250-03$
$3.48778570-03$
$3.48778810-03$
$3.48779060-03$
$3.48779200-03$
$3.48779240-03$
$3.48779270-03$
$3.48779270-03$
$3.48779270-03$
$3.48779270-03$
$3.48779270-03$

$3.50000000-0 \frac{5}{30}$

$3.49510430-05$

$3.49265860-0$

3.49021500-0

$3.48777140-05$

$3.49778420-0$

3. $4877885 \mathrm{D}-0$

3. 4877906)-0?

3.4877923)-0

3.48779277-0

$3.4877927)-0$

3.4877927000

3.48779270-0

$3.48779270-0$

$3.48779270-05$
$3.48779270-05$

$3.50000000-05$ 3.50000000-05

$000000000-05$

$3: 50000000-05$

3.50000000-05

(3000000-05

. $50000000-05$

. $500000000-05$

. $5(100000)-05$

3. $2000000-05$

. $50000000-05$

. $56000000-0$

$3.50000000-05$

. $5000000-15$

$30.5000000-05$

$.50000000-05$

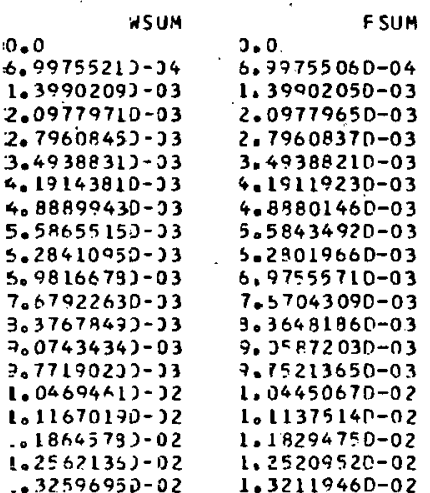

$1.32119460-02$

COMPGNENT I JEEX $=6$ NO. OF PHASES $=2$

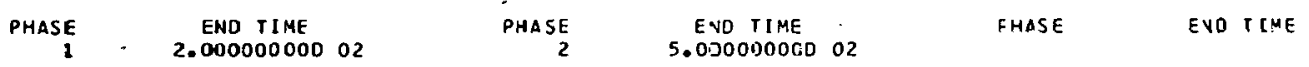

THERE ARE NO IMPOSED BDUNDARY CONDITIONS IIBPHA=CI 


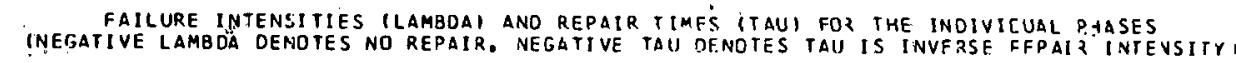
PHäÀ̉
LAMBOA
$-3.50000000-0$
-5000
0.0 TAUS
PHASE
3. $500000000-03$
T. TAll

INITIAL FAILEO PROBÁgILITY FOR PHASE 1 (000) $=0.0$

COMPONENT RELIABILITY CHARAETERISTICS

\begin{tabular}{|c|c|c|c|c|c|c|}
\hline & 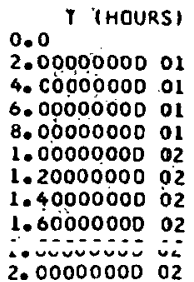 & $\begin{array}{l}0.0 \\
0.0 \\
6.59755060-04 \\
1.39902050-03 \\
2.09779650-03 \\
2.73608370-03 \\
3.49388210-03 \\
4.19119230-03 \\
4.83801460-03 \\
5.53434920-03 \\
6.97555710-03\end{array}$ & $\begin{array}{l}W \\
3.50000000-05 \\
3.49755090-05 \\
3.49510340-05 \\
3.49265770-05 \\
3.49021370-05 \\
3.48777140-05 \\
3.48533080-05 \\
3.48289190-05 \\
3.48045480-05 \\
3.4705=-20 \\
3.47553560-05\end{array}$ & $\begin{array}{l}3.50000000-05 \\
3.50000000-05 \\
3.50000000-05 \\
3.50000000-05 \\
3.50000000-05 \\
3.50000000-05 \\
3.50000000-05 \\
3.50000000-05 \\
3.50000000-05 \\
3.50000000-05\end{array}$ & 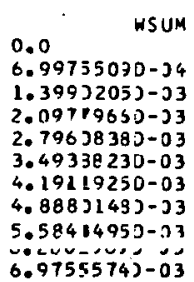 & 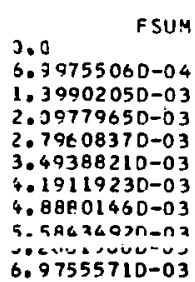 \\
\hline & 2.0000000002 & $\begin{array}{c}\text { RIGHI I I } \\
0.9 \cdot 555710-03\end{array}$ & $\begin{array}{l}\text { BOUNDARY VALUES } \\
3.47558560-05\end{array}$ & $\begin{array}{l}\text { FOR PHASE } 1 \\
\text { 3. } 50000000-05\end{array}$ & $6.9755574)-03$ & $6.97555710-03$ \\
\hline W & 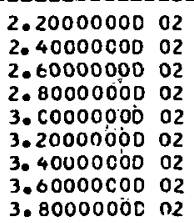 & $\begin{array}{l}7.6 \times 006630-03 \\
8.36457550-03 \\
9.05847760-03 \\
9.75177250-03 \\
1.04450670-02 \\
3.47257000-03 \\
3.4 \times 562060-03 \\
3.47929310-03 \\
3.48356070-03\end{array}$ & $\begin{array}{l}3.47315480-05 \\
3.47072400-05 \\
3.46829530-05 \\
3.46586880-05 \\
3.46344230-05 \\
3.48784600-05 \\
3.48783530-05 \\
3.48782250-05 \\
3.4878 .0750-05\end{array}$ & $\begin{array}{l}3.50000000-05 \\
3.50000000-05 \\
3.50000000-05 \\
3.50000000-05 \\
3.50000000-05 \\
3.50000000-05 \\
3.50000000-05 \\
3.50000000-05 \\
3.50000000-05\end{array}$ & 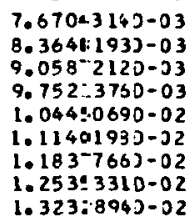 & $\begin{array}{l}7.67043090-03 \\
8.3641860-03 \\
9.05872030-03 \\
9.7521365000 \\
9.04450670-02 \\
1.044502 \\
1.11375140-02 \\
1.1829450-02 \\
1.25209520-02 \\
1.32119450-02\end{array}$ \\
\hline
\end{tabular}


MI HIMAL SET INPI:T DATA

SEI. FLAG IIPATHI. IF IPATH=1 MINIMAL CUT SETS AR IISED. IF IPATH=2 MINIMAL PATH SETS AFF USET.
FOR THIS PRUBLEM IPATH $=1$

NO. OF SETS INCUT) $=$

SET INFORMATION

SET NO. 1, HITH COMFONENTS - 13

SET NO. 2, WITH COMFCNENTS - 12

SET NO. 3, HITH COMFONENTS - $\bar{c} 3$

SET NO. 4, WITH COMFONENTS - : 6

SET NO. S, WITH COMFCNENTS - $a$ S

SET NU. 6, WITH COMFONENTS - 56 
MINIMAL SET INFOPMATION

CHARACTERISTICS FOR SET NO. =

$\begin{array}{ll} & \\ 0.0 & \text { T HOURS } \\ 2.00000000 & 01 \\ 4.00000000 & 01 \\ 6.00000000 & 01 \\ 8.00000000 & 01 \\ 8.0000000000 & 02 \\ 1.00000000 & 02 \\ 1.20000000 & 02 \\ 1.40000000 & 02 \\ 1.50000000 & 02 \\ 1.80000000 & 02 \\ 2.00000000 & 02 \\ 2.20000000 & 02 \\ 2.40000000 & 02 \\ 2.60000000 & 02 \\ 2.80000000 & 02 \\ 3.00000000 & 02 \\ 3.20000000 & 02 \\ 3.40000000 & 02 \\ 3.60000000 & 02 \\ 3.80000000 & 02\end{array}$

I (HOURS)

2.00000000 01 4.00000000 01 6.00000000 0 .0000000002 1.2000000002 1.4000000002 0.00000002 0.0000000002 2000000002 2.4000000002 0.00000000 02 3.0000000002 2000000002 3.4000000002
3.6000000002 3. 6000000002

1 (HOURS) 0.00000000 4.00000000 01 8.00000000 ol 1.2000000002

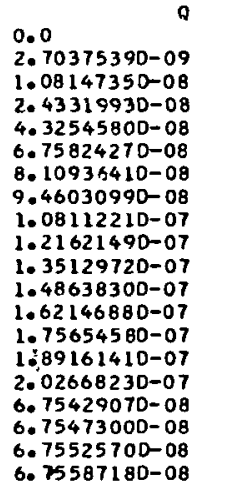

0.0
$2.70373640-10$
$5.40712130-10$
$8.11006690-10$
$1.08125730-09$
$1.35147280-09$
$1.48653750-09$
$1.62159530-09$
$1.75665470-09$
$1.89171590-09$
$2.02677010-09$
$2.16182080-09$
$2.29687150-09$
$2.43191340-09$
$2.56694660-09$
$2.70197970-09$
$1.35107770-09$
$1.35112170-09$
$1.35117430-09$
$1.35123580-09$

0.0

$2.70373640-10$
$5.40712130-10$

B. $11006710-10$

$1.08125740-09$
$1.35147290-00$

$1.48653770-09$

(1)

$.89171620-09$
$2.02677040-09$

2. $16182120-0$

2.43191350-09

$2.56694713-09$

$2.70198020-0$

$1.35107780-09$

$1.35117440-0$
$1.35123590-0$

Characteristics for Set no. =

0.0
$2.70364850-09$
$1.08145940-08$ 0

$1.08145940-08 \quad 5.70368370-10$

$2.43317820-08 \quad 8.11003180-10$

$\begin{array}{ll}6.75824270-08 & 1.08125210-09 \\ 6.35147280-00\end{array}$

$6.75771560-08 \quad 1.35142010-09$

$6.75718850-08 \quad 1.35136740-09$

$6.75683720-08 \quad 1.08353730-09$

6.75648600-08 1. $1.35129720-09$

$6.75648610-08 \quad 1.35129720-09$

$6.75648610-08 \quad 1.35129720-09$

$6.75648620-08$

$6.75648620-08$

$6.75648620-08$

$6.75648620-08$
$6.75648620-08$

$1.35129720-09$

1. $35129720-09$
1. $35129720-09$

1. $35129720-09$

1. $35129720-09$

1. $35129720-09$
1. $35129720-09$

0.0

$2.70368370-10$

$5.40709620-10$

$108125210-0$

$1.35147290-0$ \%

$1.35136750-0$

$1.35129730-09$

$1.35129730-09$

$1.35129730-09$

$.35129730-09$
$.35129730-09$

1.3512973)-09

$1.35129730-09$
$1.35129730-09$

CHARACTERISTICS FOR SET NO. $=$

0.0.
$2.70375390-09$
$1.08147350-08$
$2.43319930-08$
$4.32545800-08$
$6.75824270-08$
$8.10936410-08$
$0.49 n 2000208$

N 0.0

2. $70373640-10$

$8.11006690-10$

$1.08125730-09$

$1.48653750-09$

0.0

$5.40712130-13$

$8.11006710-10$

$1.08125740-09$

. 3514729

$1.48653770-09$

\begin{tabular}{|c|c|}
\hline UN. & SUM \\
\hline & -09 \\
\hline & \\
\hline & 433178 \\
\hline & \\
\hline & 758 \\
\hline & 9.59 \\
\hline & 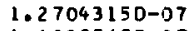 \\
\hline & \\
\hline & \\
\hline 2.3 & 2 \\
\hline & \\
\hline 3.2296705$)$ & 3.22 \\
\hline 3.70254910 & $3.70254880-07$ \\
\hline 20 & $4.20243470-07$ \\
\hline $4.72932770-07$ & $72932720-07$ \\
\hline & $3463280-07$ \\
\hline & $5.404852 E D-07$ \\
\hline $94532400-$ & $\begin{array}{l}5.67508210-07 \\
0.94532290-07\end{array}$ \\
\hline $94532400-5$ & $94532290-07$ \\
\hline
\end{tabular}

W5u4

$2.70368373-09$

$1.08(42533-09$

$2.43315710-09$
$4.3254124)-03$

$0.46103020-58$

$1.21638180-37$

$1.48655130-37$

$1.7569165)-37$
$2.02717773-07$

$2.029743715-07$
$2.5676965)-07$
$2.83795605-07$

.83795600-07

$3.1082154 J-37$
$3.3784749)-07$

$3.64873430-37$

3. 9190933$)-37$

$4.18925323-07$
$4.45051270-37$

FSUM

$2.70368370-09$ $1,08144530-08$

$2.43315710-08$
$4.32541240-0 B$

$9.45103020-08$

$1.21638180-07$

$1.75+91640-07$

$2.29743700-07$

$2.83795580-07$

3. $37847450-07$

$3.91899330-07$

$4.18025260-07$
$4 .+5951200-07$

2. 7037364$)-00$

2. $43317820-08$

4. $32544220-08$

9.59618239008
i. $37 n: 2149-17$

$2.70373640-09$

$2.43317820-08$

9. $59618280-00$
1. 7 TnM $216 n-07$ 


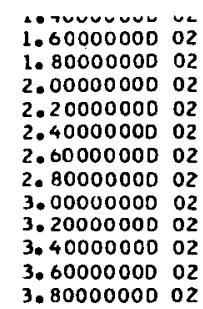

I (HOURS)

2. 00000000 o 4.00000000 01 6.0000000001 8.00000000 ol 1.2000000002 1.4000000002 1.6000000002 1.80000000 02 20000002 2. 4000000002 2.6000000002 2.80000000 02 3. 000000002 (200000 3.60000000 02 3.8000000002

0

I (HOURS)

2.000000000 4. 0000000001 8.00000000 01 1. 0000000002 1. 2000000002 1.4000000002 1.80000000 02 2. 0000000002 2. 2000000002 2.4000000002 . 2.8000000002 3. 20000000 02 3.4000000002 3.60000000 02

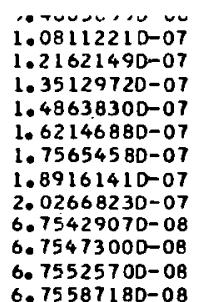

1.75665470-09 2. $02677010-09$ 2. $16182080-09$ $2.43191340-09$ 2.56694660-09 2.70197970-09 1. $35107770-09$ 1. $35123580-09$

1.7521665490-0 $1.89171620-0$
$2.026,77040-0$ $16182120-09$ $2.43191390-09$ $2.56694710-09$ $2.70198020-0$ .. $1.35117440-09$
$1.35123590-09$

CHARACTERISTICS FOR SET NO, =

0.0

..89400190-07 0

0.0.
$4.89357440-0 B$
$9.77858820-0 B$

$1.46529190-07$
$1.95165830-07$

$1,95165830-07$
$2.43717240-07$

$2.67888980-07$

$2.92043940-07$

$3,40367070-07$

$3.64513950-07$

4. $12790640-07$

4. 36907850-07

$4.61003920-07$

$4.85099990-07$

$2.42764880-07$

$2.42998550-07$

$2.43146873-07$

0.0

$.89357680-08$ . $77850733-0$ ..46529830-07 . (26789290)-07 $2.92048930-07$ (2.16209490-07 -1
0 $.36921660-07$ $4.61019603-07$ $0.43149830-07$

CHARACTERISTICS FOR SET NO० =

$n$ 0.0 7. $391603430-06$ $7.81603430-06$
$1.22072120-05$ $1.21944160-05$ 1. $21816260-05$ $1.21688820-05$ $1.21646490-05$ $1.21646660-05$ $1.216468330-05$ $1.21646930-0$ $1.21646960-05$ $1.21646980-05$ 1. $21646980-05$ $1.21646980-05$
$1.21646980-05$ $4.89229100-08$
$9.77773400-08$ $9.77773400-08$
$1.46520670-07$ $1+95(5308)-07$ $2,43717240-07$ $2.43462580-07$ $2.43377830-07$ $2.43293470-07$ $2.43293640-07$ $2.43293820-07$ $2.43293910-07$ $2.43293940-07$ $2.43293960-07$ 2. $43293960-07$ $2.43293960-07$
$2.43293960-07$ $4.89229340-08$ $9.77775310-08$
$1.45521310-07$ $1.95154603-07$ $2.43720220-07$ $2.43592890-07$ $2.43465550-07$ $2.43380790-07$ $2.43296600-07$ $2.43296730-07$ . (2) $2.43296920-07$ $2.43294920-07$
$1060825663-07$ $1.07309363-07$ 2. $36494225-37$ 2.78380135- $4.2024351\}-07$ 4.72932770-07 $5.67508330-37$ 0.0
$4.8935744)-37$ $1.05657370-35$ 4. $39972440-06$ 1.7321563)$2.90033750-35$ . $0.68609510-35$ $7.56400730-05$ (2) $.02094930-04$
$.06955280-34$

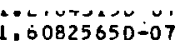
$1.97309360-07$ $2.36494210-07$ . $22067040-07$ 3.70254880-07 4. $20243470-07$ $4.12932720-07$
$5.13463280-07$ $5.40<85260-07$ $5.37508210-07$

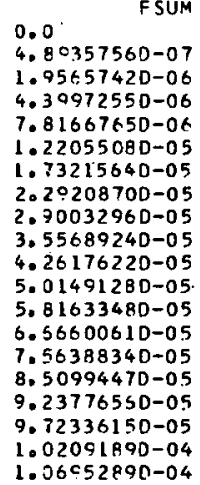

4. $089220100-37$ NS.JM $2.43296920-07$
$2.43296920-07$ $1.9562316)-06$
$4.39921170-36$ .8159492J-J5 $.2204652 J-05$
$.70777240-35$ 2. $19482490-05$ $2.6816 E 530-05$ 3.6550077)-05 $4.14159503-05$ $4.62818250-05$ $5.50135810-05$ $7.06112130-35$ $7.54770970-05$
$8.03429770-05$
4. $89229220-07$ 1. $55623210-06$ 7. E1595110-06 1. $22046550-05$ $2.19482040-05$ 2. 6 P $165490-05$ 3. $65497850-05$ $4,14155260-05$ $4.62812460-05$ $5.11469460-05$ 6.087.82 760-05 $6.57439070-05$
$7.06095140-05$ $7.54750970-05$
$8.03406560-05$ 

0.07000001 4.00300000001 6. C00000000' 01 8.00000000001 $1.2050000 \mathrm{C} 02$ 1.4030000002 $1.6000000 \mathrm{D}=02$ 1.80000000 .02 2. 2000000002 2.40000000 .02 $2.60000000^{\circ} 02$ $3.00000000 \cdot 02$ 3. $00000000 \cdot 02$ 3.40000000 O2 3.6000000002
3.8000000002
0

4. $894001.90-07$

1. $95601530-06$

$4.40023760-06$
$7.81705900-06$

$1.22012120-05$

$1.46358550-05$

$1 ., 70602390-05$

$1.94837900-05$

$2.43292480-05$

$2.675155600-05$

$2.9173888 \mathrm{D}-05$

3. $15940860-05$

$3.40421500-05$

$1.21116040-05$

l. $21222760-05$

$1.21350530-05$
$1.21409380-05$

0.0
$4.89357440-08$
$9.77858820-08$
$1.46529190-07$
$1.95165830-07$
$2.43717240-07$
$2.06788980-07$
$2.92043940-07$
$3.16203330-07$
$3.40367070-07$
$3.64513950-07$
$3.88652300-07$
$4.072790640-07$
$4.36907850-07$
$4.51003920-07$
$4.35099990-07$
$2.42764880-07$
$2.42871220-07$
$2.42998550-07$
$2.43146870-07$

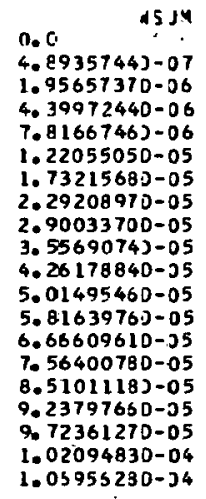

0.0

$4.89357560-0.7$

$1.95657420-0.6$

$4.39972550=06$

$1.22055080-05$

$2.29208700-05$

$2.90032950-05$

$4.26176220-05$

$4.26176220-05$
$5.01491230-05$

5. $81533490-05$

$6.66500610-05$

$7.56388340-05$

$9.23776560-05$

$9.723361500-05$

$1.02091850-04$ 
SYSTEM INFORMATION-UPPER BOUNDS

DIFFERENTIAL CHARACTERISTICS-UPPER BDUNDS T ( HUURS)

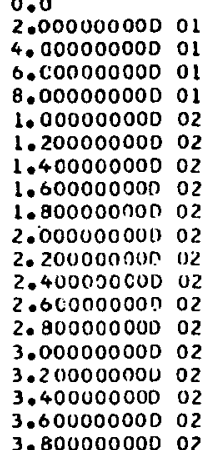

0.0

$$
\begin{aligned}
& 0.0 \\
& 1.476054180-06 \\
& 5.902837560-06 \\
& 1.327313700-05 \\
& 2.357972030-05 \\
& 3.682393000-05 \\
& 4.169530670-05 \\
& 4.655910340-05 \\
& 5.142362070-05 \\
& 5.629424190-05 \\
& 6.115976440-05 \\
& 6.403124760-05 \\
& 7.090272070-05 \\
& 7.576994100-05 \\
& 8.063287910-05 \\
& 8.540580630-05 \\
& 3.559010860-05 \\
& 3.561145540-05 \\
& 3.663702480-05 \\
& 3.666680480-05
\end{aligned}
$$

$0.076055140-07$

$2.949712370-07$

$4.420120600-07$

$5.887285120-07$

$7.352061530-07$
$7.836923780-07$

$8.321450290-07$

$8.806491350-07$
$9.292045410-07$

$9.777262040-07$

$1.026273170-06$

$1.123324740-06$

$1.171786970-06$

$7.328771790-07$

$7.330899450-07$
$7.333447110-07$

$7.333447110-07$
$7.336414760-07$
$0.0 \quad 2$ $1.476057310-07$
$2.949729780-07$ 4. $420179270-07$ 7. 352332270-07 7.837250560-07 8. 806944230-07 3.272568520-07 $3.777860050-07$
$1.026340940-06$ $1,026340940-06$
$1,074896360-06$ $1.123409860-06$ 1,171881 $7.329039960-07$ $7.334167860-07$
$7.333715790-07$ $7.333715790-07$
$7.336683780-07$

I (HOURS)

INTEGRAL CHARACTER IST IES-UPPER GOUNOS

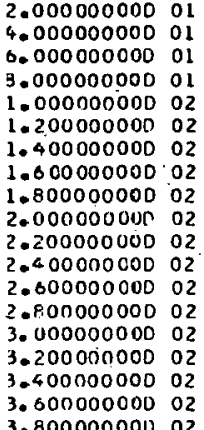

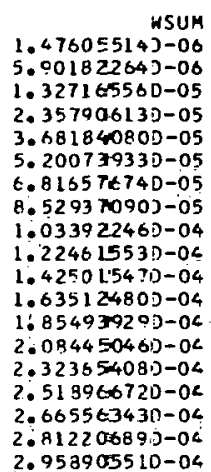

FSUY
$1.476056230-06$

$5.901826973-06$

2.3579078r0-35

$3.681843513-05$

$5.200734330-05$
$6.816546070-05$

$8.529292830-05$

$1.03390734)-04$

$1.224590090-04$

$1.53506781 J-04$

$2.323514770-04$

$2.5187932+0-34$

$2.66535731)-0$

2. $958627650-94$

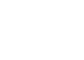


SYSTEM INFORMATION-BEST BRACKETS

DIFFERENTIAL :HARACTERISTICS-BEST BRACKETS

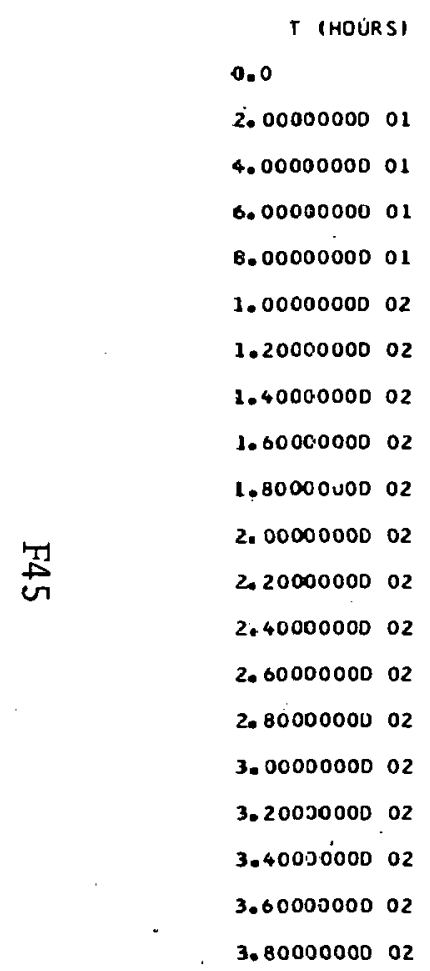

OMIN

0.0

$1.47537010-06$

5.89737220-06

7. 32547280-05

2. 35361880-05

$3.67390410-05$

4. $15936190-05$

$4.64397330-05$

5. 12884610-05

$5.61423460-05$

$6.09911680-05$

$6.58659310-05$

7.07007020-05

$7.55512290-05$

$8.03975110-05$

$8.52437920-05$

3. $65060290-05$

$3.65273060-05$

3.65527830-05

3.65824600-05
QMAX

$1.47537010-06$

$5.89737220-08$

1. 3254728D-05

$2.35361860-05$

3.67390410-05

4.15936190-05

$.64397330-05$

5.12884610-05

$5.61423460-05$

$6.09911680-05$

$0.58459310-05$

7.07007020-05

$7.55512290-05$

8.03975110-05

8. 52437920-05

3.65060290-05

3.65273060-05

$3.65527830-05$

$3.65824600-05$
0.0

$1.47502780-07$

$2.94560700-07$

4.41089540-07

$5.87090760-07$

7.326.50250-07

7.80798930-07

$8.28314630-07$

9. $25299490-07$

$9.73483990-07$

$1.02169340-06$

$1.06990290-06$

$1.11807010-06$

1. $16619530-06$

$1.21432050-06$

$7.30337550-07$

7. 30548830-07

$7.30801810-0$

$7.31076510-07$
0.0

$1.47502780-07$

$2.94560700-07$

$4.41099540-07$

$5.87090750-07$

7. 32650250-07

7.80799930-57

8. 23914630-07

8.77081590-07

9.2529949D-07

$9.73483990-57$

$1.02169340-06$

$1.08990280-06$

1.11807010-05

$1.16619530-06$

1. $21432050-06$

7.30337550-07

$7.3054883 D-07$

7.3080181ว-07

7.31096510-0T

\begin{tabular}{|c|c|}
\hline LuIV & LMAX \\
\hline 0.0 & 0.0 \\
\hline $1.47503005-07$ & $1.47503000-07$ \\
\hline $2.94552430-07$ & $2.945622430-07$ \\
\hline $4.41 .0 \equiv 5390-07$ & $4.41095390-07$ \\
\hline $5.87104590-07$ & $5.87104580-07$ \\
\hline $7.32577160-07$ & $7.32677160-07$ \\
\hline $7.83231410-07$ & $7.80831410-07$ \\
\hline $9.24553120-07$ & $8.28953120-07$ \\
\hline P. $87125599-07$ & $8.77126580-07$ \\
\hline $9.25331+\div 0-07$ & $9.25351440-07$ \\
\hline $9.73543370-07$ & $9.73543370-07$ \\
\hline $1.02176070-05$ & $1.02176070-06$ \\
\hline $1.05737840-05$ & $1.06997840-06$ \\
\hline $1.11815450-05$ & $1.11815450-06$ \\
\hline $1.16023910-06$ & $1.16628910-06$ \\
\hline $1.21+52+50-05$ & $1.21442400-06$ \\
\hline $7.30354210-07$ & $7.30364210-07$ \\
\hline & $10-07$ \\
\hline $7.30323530-07$ & 7 \\
\hline $7.3112325 D-07$ & $7.31123250-07$ \\
\hline
\end{tabular}

$\Delta x$

7. 3112325D-07

T. 31123250-07 
FAILURE RATE CONTRIBUTIONS

I (HOURS)

0.0

2.0000000001

4.00000000 01

6.0000000001

. 8.0000000001

1.0000000002

1. 2000000002

1.4000000002

1.6000000002

1.8000000002

2.0000000002

2. 2000000002

2.4000000002

2.6000000002

2.8000000002

3.0000000002

3.2000000002

$3.40000000 \cdot 02$

3.60000000 .02

3.8000000002
WIMIN

0.0

MIMAX

0.0

$1.47554150-07$

$2.94765980-07$

$4.41550830-07$

5. 87909710-0.7

7.33928350-07

7. ค2245830-07

8. 30530040-07

8.78865610-07

9. 27252300-07

9. $75605430-07$

1. $02398360-06$

$1.07236190-06$

1.12069790-06

1. $16809170-06$

1. $21723540-06$

7. 31607510-07

7. 31819530-07

.7. 32073410-07

7. 3236914D-07
$5.13655600-11$

$2.94765980-07 \quad 2.05290130-10$

$4.41550830-07 \quad 4.61291730-10$

5.87909710-07 8.19949610-10

$7.33928350-07$

$7.82245830-07$

8. 3053004D-07

8.78865610-07

$9.27252300-07$

9.75605430-07

$1.02398360-06$

$1.07236190-06$

$1.12069790-06$

$1.16899170-06$

$1.21728540-06$

7.31607510-07

7.31819530-07

7. 32073410-.07

$7.32369140-07$ w2max 0.0

WMIN

0.0

5.13655600-11 $1.47502780-07$

$2.05280130-10$

$4.61291730-10$

8. $187495610-10$

$1.27810180-09$

$1.44690230-09$

$1.61541270-09$

$1.78401980-09$

$1.95281220-09$

2. $12143300-09$

$2.29026640-09$

$2.45910530-09$

2. $62780110-09$

$2.79635380-09$

$2.96491160-09$

$1.26996230-09$

1.27070550-09

$1.27150550-29$

$1.27263210-09$
$2.94550700-07$

4. 41089540-07

5.87090750-07

7. 32550250-07

7. 807989930-07

B. 237314530-07

8.77081590-07

9.25299490-07

$9.73+33370-37$

1. $02159340-06$

$1.05390280-05$

1.11607010-06

1. $16619530-06$

1. 21432050-05

7.30337550-07

7. $30548830-07$

7. 305J1810-07

7. $31075510-07$
0.0

$1.47502780-07$

2. $94560700-07$

4. 41089540-07

5.87090760-07

7. 32650250-07

7. $80798930-07$

8.28914630-07

$9.77081590-07$

$9.25299490-07$

9.73483990-07

$1.02169340-06$

$1.06990280-06$

$1.11807010-06$

1. 16619530-0t

1.21432050-00

7. 30337550-07

7.30548930-07

7. 30801810-07

7. 31096510-07 
DIFFERENTIAL CHARACTËRISTIICS-LAST BRACKETS

\begin{tabular}{|c|c|c|c|c|c|}
\hline T IHOUR & & OL AST & HILASST & W2MIN-LAST & $W 2 M A X-L A S T$ \\
\hline 0.0 & & 0.0 & 0.0 & 0.0 & 0.0 \\
\hline$\overline{2}_{0.00000000}$ & 01 & $1.47537010-06$ & $1.4755 .41 .50-07$ & $5.13655600-11$ & $5.13355630-11$ \\
\hline 4.00000000 & 01 & $5.89737220-05$ & $2.54765980-07$ & $2.05280130-10$ & $2.05280130-10$ \\
\hline 6.000000000 & 01 & $1.32547280-05$ & $4.41550830-07$ & $4.61291730-10$ & $4.51291730-10$ \\
\hline 8.00000000 & ol & $2.35361860-05$ & $5.87909710-07$ & $8.18949610-10$ & $8.13949610-10$ \\
\hline 1.00000000 & 02 & $3.67390410-05$ & $7.33928350-07$ & $1.27910180-09$ & $1.27310190-25$ \\
\hline 1.20000000 & 02 & $4.15936190-05$ & $7.82245830-07$ & $1.44690230-09$ & $1.44690230-09$ \\
\hline 1.40000000 & 02 & $4.64397330-05$ & $8.30530040-07$ & $1.51541270-09$ & $270-05$ \\
\hline 1.60000000 & 02 & $5.12834610-05$ & $8.78865610-07$ & $1.78401980-09$ & $1.73401790-09$ \\
\hline 1.80000000 & 02 & $5.61423460-05$ & $9.27252300-07$ & $1.95281220-09$ & $1.95281220-09$ \\
\hline 2.00000000 & 02 & $6.09911680-05$ & $9.75605430-07$ & $2.12143300-09$ & $2.12243300-05$ \\
\hline 2.20000000 & 02 & $6.58459310-05$ & $1.02398360-06$ & i. $29.026640-09$ & $2.29026640-09$ \\
\hline 2.40000000 & 02 & $7.07007020-05$ & $1.07236190-06$ & $2.45910530-0^{\circ}$ & $2.45910530-09$ \\
\hline 2.50000000 & 02 & $7.55512290-05$ & $1.12069790-06$ & $2.62780110-09$ & $2.52733110-39$ \\
\hline 2.90000000 & 02 & $8.03975110-05$ & $1.16899170-06$ & $.2 .79835380-00$ & $2.79635380-09$ \\
\hline 3.00000000 & 02 & $8.52437920-05$ & $1.21729540-08$ & $2.96491160-09$ & $2.75491160-39$ \\
\hline 3.20000000 & 02 & $3.65060290-05$ & $7.31607510-07$ & $1.26996230-00$ & $1.25995230-09$ \\
\hline 3.40000000 & 02 & $3.65273060-05$ & $31819530-07$ & $1.27073550-00$ & $1.27070550-09$ \\
\hline 100 & 02 & 3.6552783 & $3410-07$ & $1.27159550-09$ & 1.27 \\
\hline 3.90000000 & 02 & $3.65824600-05$ & $7.32360140-07$ & $1.27263210-09$ & $1.27263210-09$ \\
\hline
\end{tabular}


INTEGRAL CHARACTER ISTICS-BEST BRACKETS

\begin{tabular}{|c|c|c|c|c|c|}
\hline T IHOUR & & MMIN-SUM & UMAX-SIJM. & FMIN-SUM & FMAX-SUM \\
\hline 2.00000000 & 01 & $1.47502780-06$ & $1.47502780-06$ & $1.4750289[-06$ & $1.67502370-0.06$ \\
\hline 4.00000000 & 01 & $5.89566260-06$ & $5.89566260-06$ & $5.8956670[-06$ & $5.89566700-06$ \\
\hline 6.00000000 & 01 & $1.32521650-05$ & $1.32521650-05$ & $1.32521756-05$ & $1.32521750-05$ \\
\hline 8.00000000 & 01 & $2.35339680-05$ & 2.353396 AD -05 & $2.35339885[-05$ & 2. 35339850-05 \\
\hline 1.00000000 & 02 & $3.67313780-05$ & $3.67313780-05$ & $3.6731405 C-05$ & $3.67314050-05$ \\
\hline 1.20000000 & 02 & $5.18658700-05$ & $5.18658700-05$ & 5. $1865820[-05$ & $5.18359200-35$ \\
\hline 1.40000000 & 02 & $0.79630060-05$ & $6.79630060-05$ & $6.79627015-05$ & $6.79627010-05$ \\
\hline 1.60000000 & 02 & $8.50229680-05$ & 8. $50229680-05$ & $8.53221935-05$ & $8.53221930-05$ \\
\hline 1.80000000 & 02 & $1.03046780-04$ & $1.03046780-04$. & $1.0304528[-04$ & $1.23345280-04$ \\
\hline 2. 000000000 & 02 & $1.22034610-04$ & $1.22034610-04$ & $1.2203209[-04$ & 1. 22032093-04 \\
\hline $2.20000000^{\circ}$ & 02 & $1.41986390-04$ & $1.41986390-.04$. & $1.4198250[-04$ & $1.41982500-04$ \\
\hline 2.40000000 & 02 & $1.62902350-04$ & $1.62902350-04$. & $1.6289670[-04$ & $1,62895700-04$ \\
\hline 2.60000000 & 02 & $1,84782080-04$ & $1.84782080-04$ & $1.8477422[-04$ & $1.84774220-04$ \\
\hline 2.80000000 & 02 & $2.07624730-04$ & $2.07624730-04$ & $2.07616180-04$ & $2.07514180-04$ \\
\hline 3.00000000 & 02 & $2.31429890-04$ & $2.31429890-04$ & $2.3141608[-04$ & $2.31416080-04$ \\
\hline 3.20000000 & 02 & $2.50876470-04$ & $2,50876470-04$. & $2.5085928[-04$ & $2.538592880-04$ \\
\hline 3.40000000 & 02 & $2.65485330-04$ & $2.65485330-04$ & $2.65464900-04$ & $2.654649900-04$ \\
\hline 3.60000000 & 02 & $2.80098840-04$ & $2.80098840-06$ & $2.3007496 r .04$ & $2.80374950-04$ \\
\hline 3.8000000 & 02 & $2.94717825-04$ & $2.94717820-04$ & $2.9469027 r .-04$ & $2.76530270-04$ \\
\hline
\end{tabular}

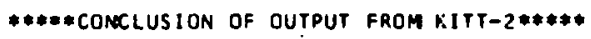




\section{APPENDIX G}

\section{REQUIRED JOB CONTROL LANGUAGE FOR THE} EXECUTION OF. PREP AND KITT AT THE NRTS

The use of the PREP and KITT computer codes on the IBM $360 / 75$ computer at the NRTS requires minimal Job Control Language. In order to use PREP and/or KITT, the user need only supply a standard NRTS job card, one of the four EXEC cards described below, and any required input data.

To run the PREP codes in order to obtain the fault tree's minimal cut or path sets, the following EXEC card should be used.

\section{// EXEC PREP}

If the user expects the running time will exceed 5 minutes, the card should be coded as,

\section{// EXEC PREP,PTIME=XXX}

where $X X X$ is to be replaced by the time required in minutes (left justified). If the KITT-1 program is to be used to. obtain the probability information from the fault tree then the following EXEC card should be supplied.

\section{// EXEC KITTI}

If the time required to evaluate the fault tree will exceed 5 minutes, then this card should be coded as follows.

\section{// EXEC KITTI, KTIME=XXX}

To use the KITT-2 program to obtain the probability characteristics the EXEC card is coded as

$$
\text { // EXEC KITT2, KTIME=XXX }
$$


where KTIME=XXX may be omitted if the execution time will be less than or equal to 5 minutes.

In addition to the above procedures for the execution of any one of the three components of the code package a procedure is available which allows the automatic sequential use of PREP followed by KITT-1. That is, PREP is first executed to obtain the fault tree's minimal cut or path sets, and then these sets are passed automatically to KITI-l which obtains the associated probabilistic information. The use of this automatic link precludes the running of several parameter runs with KITT-I (ie, NPROB = I only). This automatic link is executed with the following input deck arrangement.

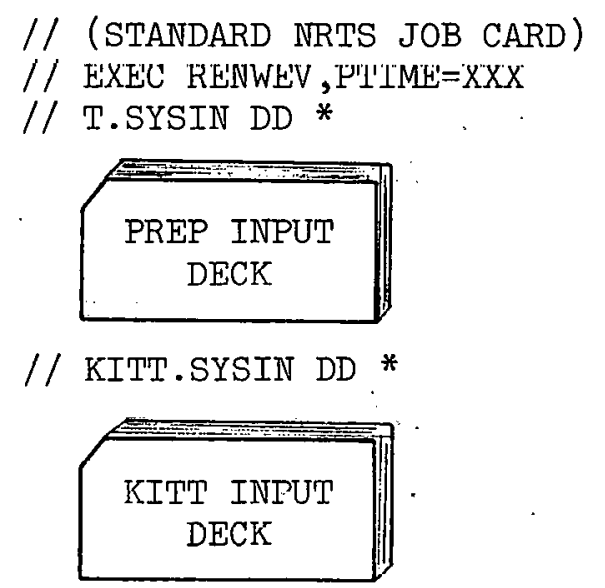

The input deck for KITT-I is the same as that used when KITT-1 is executed alone except that the cards which specify the number of components (NCOMP), the failure intensities, repair times, the number of minimal cut or path sets (NCUT), and the minimal cut or path sets will be supplied automatically by PREP. These cards must be omitted f'rom the deck supplied by the user. 